\title{
Imaging myocellular lipid droplet dynamics in relation to insulin sensitivity
}

Citation for published version (APA):

van Polanen, N. (2021). Imaging myocellular lipid droplet dynamics in relation to insulin sensitivity: Analysis in human intervention studies. [Doctoral Thesis, Maastricht University]. Maastricht University. https://doi.org/10.26481/dis.20210922np

Document status and date:

Published: 01/01/2021

DOI:

10.26481/dis.20210922np

Document Version:

Publisher's PDF, also known as Version of record

\section{Please check the document version of this publication:}

- A submitted manuscript is the version of the article upon submission and before peer-review. There can be important differences between the submitted version and the official published version of record.

People interested in the research are advised to contact the author for the final version of the publication, or visit the DOI to the publisher's website.

- The final author version and the galley proof are versions of the publication after peer review.

- The final published version features the final layout of the paper including the volume, issue and page numbers.

Link to publication

\footnotetext{
General rights rights.

- You may freely distribute the URL identifying the publication in the public portal. please follow below link for the End User Agreement:

www.umlib.nl/taverne-license

Take down policy

If you believe that this document breaches copyright please contact us at:

repository@maastrichtuniversity.nl

providing details and we will investigate your claim.
}

Copyright and moral rights for the publications made accessible in the public portal are retained by the authors and/or other copyright owners and it is a condition of accessing publications that users recognise and abide by the legal requirements associated with these

- Users may download and print one copy of any publication from the public portal for the purpose of private study or research.

- You may not further distribute the material or use it for any profit-making activity or commercial gain

If the publication is distributed under the terms of Article $25 \mathrm{fa}$ of the Dutch Copyright Act, indicated by the "Taverne" license above, 


\section{Imaging myocellular lipid droplet dynamics in relation to insulin sensitivity}

Analysis in human intervention studies

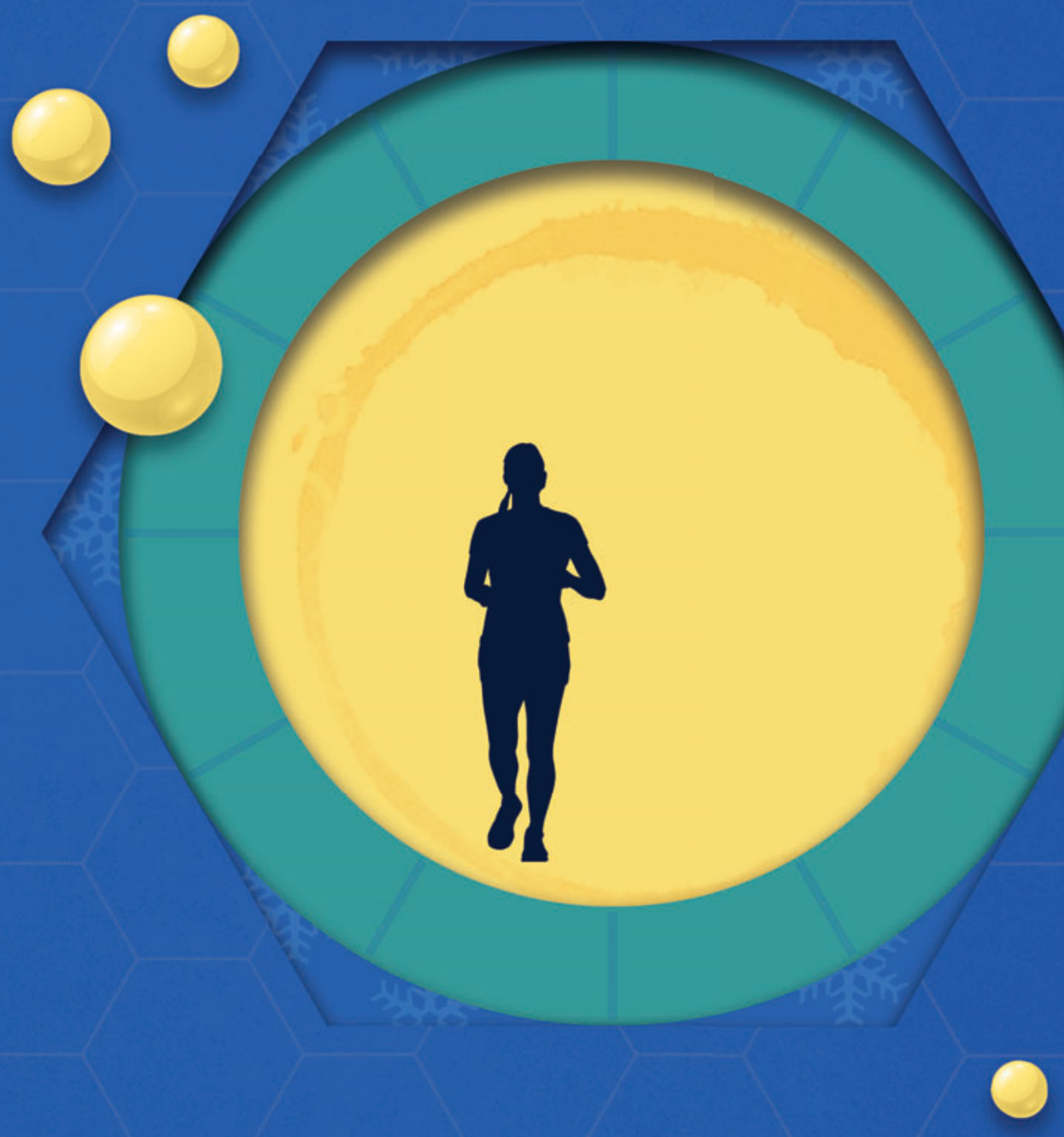

Nynke van Polanen 



\title{
Imaging myocellular lipid droplet dynamics in relation to insulin sensitivity
}

\author{
Analysis in human intervention studies
}

Nynke van Polanen 


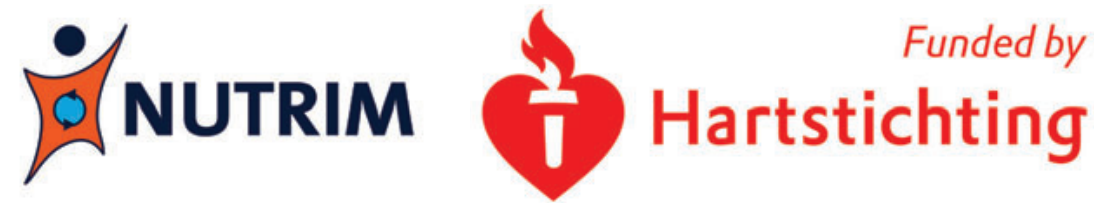

The research presented in this thesis was performed within NUTRIM School of Nutrition and Translational Research in Metabolism. The research described in this thesis was supported by a grant of the Dutch Cardiovascular Research Initiative: an initiative with support of the Dutch Heart Foundation (CVON2014-02 ENERGISE).

Copyright $@$ Nynke van Polanen, Maastricht 2021

Cover design: Evelien Jagtman | www.evelienjagtman.com

Layout: Nynke van Polanen

Printing: Ridderprint | www.ridderprint.nl

ISBN: 978-94-6416-684-2 


\section{Imaging myocellular lipid droplet dynamics in relation to insulin sensitivity}

Analysis in human intervention studies

\section{DISSERTATION}

to obtain the degree of doctor at Maastricht University, on the authority of the Rector Magnificus,

Prof. Dr. Rianne M. Letschert

in accordance with the decision of the Board of Deans,

to be defended in public on

Wednesday 22nd of September, 2021 at 13:00 hours

$$
\text { by }
$$

\section{Nynke van Polanen}

Born in Almere, the Netherlands, on the $7^{\text {th }}$ of June, 1990 


\section{Promotors}

Prof. Dr. M.K.C. Hesselink

Prof. Dr. P. Schrauwen

\section{Copromotor}

Dr. A. Gemmink

\section{Assessment committee}

Prof. Dr. R. Shiri-Sverdlov (Chair)

Prof. Dr. M.A.M.J. van Zandvoort

Prof. Dr. Ir. J. Keijer (Wageningen University \& Research)

Dr. S.O. Shepherd (Liverpool John Moores University, UK)

Dr. R.C.R. Meex 


\section{Table of contents}

$\begin{array}{lll}\text { Chapter } 1 & \text { General introduction } & 7\end{array}$

Chapter 2 The effect of diet and exercise on lipid droplet dynamics in human muscle tissue

Chapter 3 Acute cold exposure leads to increased levels of triglycerides during mixed-meal tests in young healthy subjects

Chapter 4 Circadian misalignment disturbs the skeletal muscle lipidome in healthy young men

Chapter 5 Resveratrol-induced remodelling of myocellular lipid stores: a study in metabolically compromised humans

Chapter 6 Post-exercise changes in myocellular lipid droplet 153 characteristics of young lean individuals are affected by circulatory non-esterified fatty acids

Chapter 7 A novel microscopy tool to study lipid droplet dynamics 179 in vitro in human primary myotubes

Chapter 8 General discussion

Appendix Impact paragraph

Samenvatting

Dankwoord

List of publications 


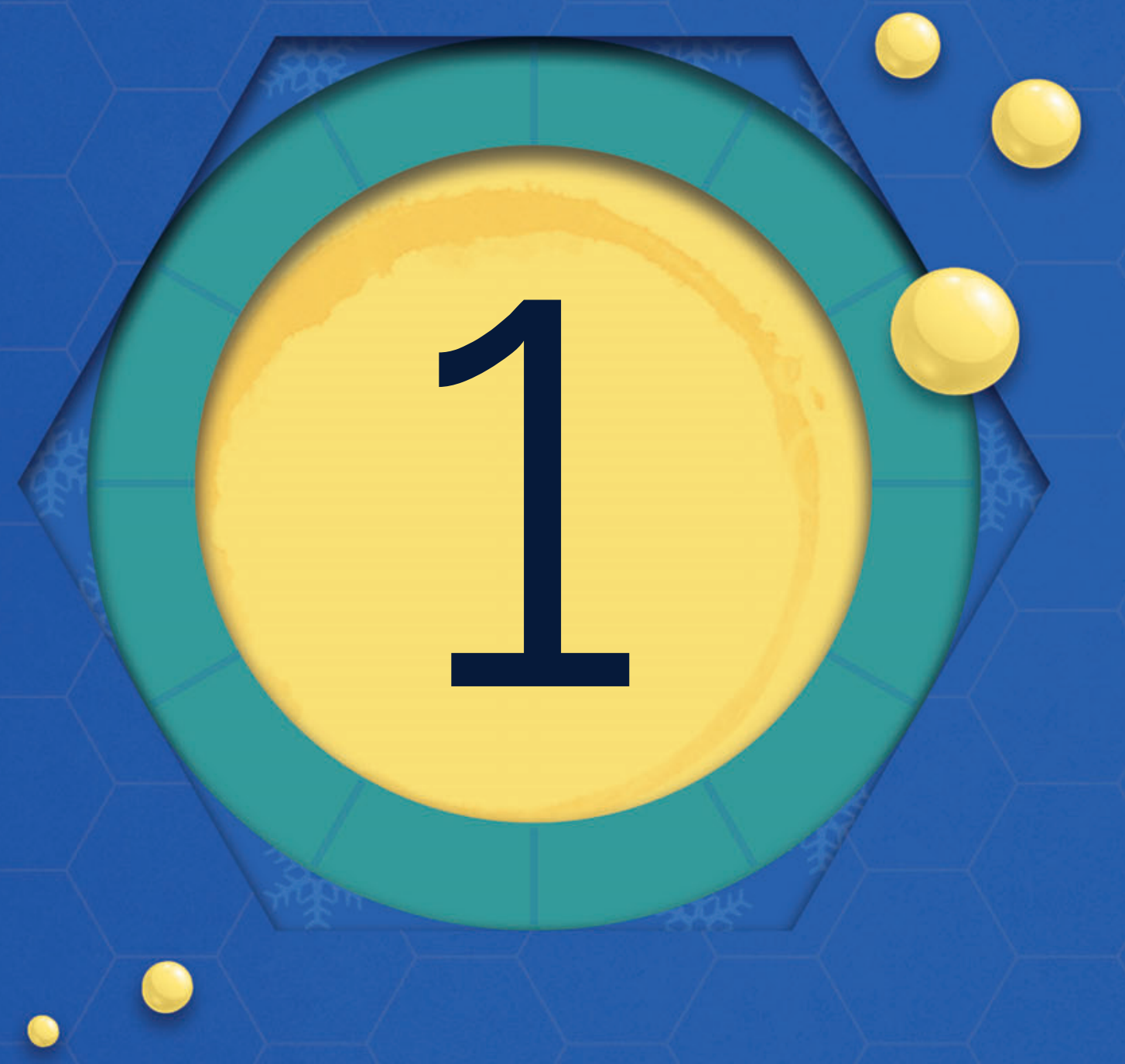


General introduction 



\section{Obesity, type 2 diabetes and insulin resistance}

In $2019,50 \%$ of the Dutch population was overweight (BMI $\left.>25 \mathrm{~kg} / \mathrm{m}^{3}\right)$ and $15 \%$ obese $\left(\mathrm{BMI}>30 \mathrm{~kg} / \mathrm{m}^{3}\right)$ (Volksgezondheidenzorg.info, 2020a). Obesity is an important risk factor for the development of type 2 diabetes mellitus (T2DM) and the majority of the population that has diabetes is overweight or obese (Vazquez et al., 2007; Laaksonen et al., 2010; Schnurr et al., 2020). Approximately 1.2 million people have been diagnosed with diabetes, from which 90\% suffers from T2DM (Volksgezondheidenzorg.info, $2020 \mathrm{~b}$ ). Diabetes negatively affects quality of life and is amongst the top 3 of diseases with the highest disease burden (Volksgezondheidenzorg.info, 2020c). Patients with T2DM often display co-morbidities such as renal problems, retinopathy, cancer and cardiovascular diseases (Deshpande et al., 2008; Zheng et al., 2018).

Patients with T2DM are diagnosed by high plasma glucose levels (hyperglycaemia). The exact mechanism of the development of T2DM is not known, but patients with T2DM are characterized as insulin-resistant. Insulin resistance is described as the reduced responsiveness of the peripheral tissues to insulin. This will lead to a reduction of glucose uptake by the peripheral tissues and blunted inhibition of glucose production by the liver, resulting in hyperglycaemia (Cersosimo et al., 2000). Impeded insulin sensitivity is initially compensated for by increased insulin secretion, resulting in high plasma insulin levels, and insulin resistance is a major factor in the development of T2DM. Muscle insulin resistance is seen as the first hallmark of developing T2DM (DeFronzo \& Tripathy, 2009). While the precise origin of reduced muscle insulin sensitivity remains to be elucidated, the conventional hypothesis reads that insulin signalling is impeded via bioactive lipids that may accumulate in the muscle of obese individuals (Petersen \& Shulman, 2018). Examples of these bioactive lipids are specific isoforms of diacylglycerols (DAGs) and ceramides. A possible mechanism is the bioactive lipid-mediated activation of protein kinase $C$, a serine kinase with inhibitory effects on insulin signalling (Itani et al., 2002; Szendroedi et al., 2014). This links muscle insulin resistance to fat metabolism. Therefore, studies modulating fat metabolism in muscle are warranted in an attempt to improve insulin sensitivity. In this thesis, the focus will predominantly be on factors that modulate 


\section{Chapter 1}

fat storage and to a lesser extent on fat oxidation.

\section{Glucose and fat metabolism}

The majority (about $80 \%$ ) of postprandial glucose uptake resides in skeletal muscle and is insulin-dependent. Insulin does, however, not only regulate glucose metabolism, but is also involved in lipid metabolism. Insulin inhibits lipolysis and stimulates lipogenesis and therefore can have profound effects on non-esterified fatty acids (NEFAs) in the circulation. Although lipids are mainly stored in the adipose tissue, the uptake and storage of fatty acids (FAs) also takes place in non-adipose tissue like liver, heart and skeletal muscle, a process referred to as ectopic lipid storage.

Adipose tissue is mainly responsible for the production of NEFAs in the plasma, while the liver provides triacylglycerols (TAGs) in the form of very low-density lipoprotein (VLDL) in the plasma (Figure 1). VLDL derived FAs are mostly taken up by the adipose tissue after activation of the lipoprotein lipase or in a smaller proportion in skeletal muscle. The NEFAs taken up by the muscle can be oxidized in mitochondria and excess lipids are re-esterified into TAGs and stored in numerous small lipid droplets (LDs), making up the intramyocellular lipid (IMCL) pool.

\section{Lipid droplets}

Lipids in the muscle are stored in the form of LDs. LDs are dynamic organelles containing mostly TAGs and cholesterol esters surrounded by a phospholipid monolayer (Figure 2) (Farese \& Walther, 2009). LDs form the total IMCL pool and continuously undergo lipid uptake, storage and release for oxidation. The capacity of LDs to adjust to changes in nutritional status or energy demand demonstrate the dynamic nature of the LDs and is referred as LD dynamics (Badin et al., 2013). LDs can vary in number, size and subcellular distribution. The total muscle IMCL pool is the product of the size and number of LDs. Thus, with a similar level of IMCL, the lipids can be dispersed in numerous small LDs or in fewer albeit larger LDs. Furthermore, IMCL content differs between muscle fibre types, where type I muscle fibres have a higher IMCL content compared with type II muscle fibres 
(Essén et al., 1975; Loon et al., 2004; Schrauwen-Hinderling et al., 2006). Type I fibres are oxidative muscle fibres containing a high number of mitochondria and are highly vascularized, hence better equipped for lipid oxidation. Type II muscle fibres are, on the other hand, more glycolytic, containing less mitochondria and have a lower capillary supply. Subcellularly, one can find LDs just adjacent to the sarcolemma (subsarcolemmal LDs) or dispersed alongside the myofibrils (intermyofibrillar LDs).

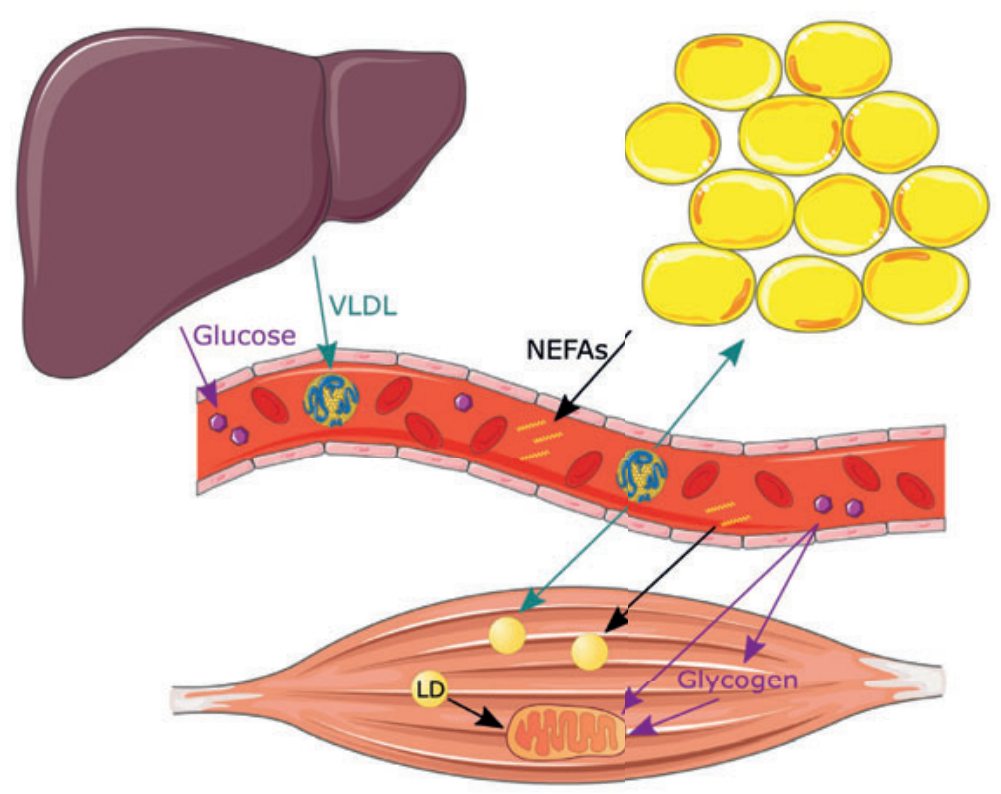

Figure 1. Overview of lipid and glucose exchange between liver, adipose tissue and the muscle. Glucose enters the bloodstream via the intestines or is released by the liver. It is mostly taken up by the muscle where it can be stored as glycogen or oxidized by mitochondria. Triacylglycerols (TAGs) are present in the plasma in the form of very low-density lipoprotein (VLDL), which is produced by the liver, or as chylomicrons from the intestine (not shown). VLDL derived TAGs are taken up by the adipose tissue and muscle. The adipose tissue can release non-esterified fatty acids (NEFAs) into the bloodstream and are subsequently taken up by skeletal muscle. The muscle stores NEFAs as TAGs in lipid droplets (LDs) which can then again liberate NEFAs and make these available to the mitochondria for oxidation. Most schematic art pieces from this figure were obtained from Servier Medical Art, which are licensed under a Creative Commons Attribution 3.0 Unported License; https://smart.servier.com. 


\section{Chapter 1}

Proteins can play a role in LD dynamics and LDs in the muscle tissue are decorated with different proteins. Examples are lipolytic enzymes, but also proteins of the perilipin (PLIN) family. The most studied member of the PLIN family is PLIN5, present in oxidative tissue such as muscle tissue. The role of PLIN5 is not fully understood; a dual role has been proposed in both lipid storage and lipid oxidation, depending on demand, nutrition or training status (Bosma et al., 2012; Bosma et al., 2013; Gemmink et al., 2016; Shepherd et al., 2017). PLIN5 has also been found on the LD-mitochondria junction, possibly involved in the flux of lipids between LDs and mitochondria (Gemmink et al., 2018).

LDs are important storage sides for neutral lipids, such as TAGs. During lipolysis, TAGs are broken down in DAGs by the lipolytic enzyme adipose triglyceride lipase (ATGL) under the release of FAs. DAGs can be further broken down to monoacylglycerols by the lipolytic enzyme hormone sensitive lipase (HSL). The final step of lipolysis is the formation of glycerol and FAs by monoglyceride lipase. Lipids can be metabolised for oxidation or used for the generation of other lipid species, necessary for membrane structure or other cellular processes. The bioactive intermediates of lipid metabolism, such as NEFAs, DAGs or ceramides are considered toxic to the cell (Liu et al., 2007; Park et al., 2008; Verma et al., 2014), referred to as lipotoxicity. Therefore, the capacity to store lipids as inert TAGs in LDs is suggested to be protective against lipotoxicity (Listenberger et al., 2003; Rambold et al., 2015; Nguyen et al., 2017). 


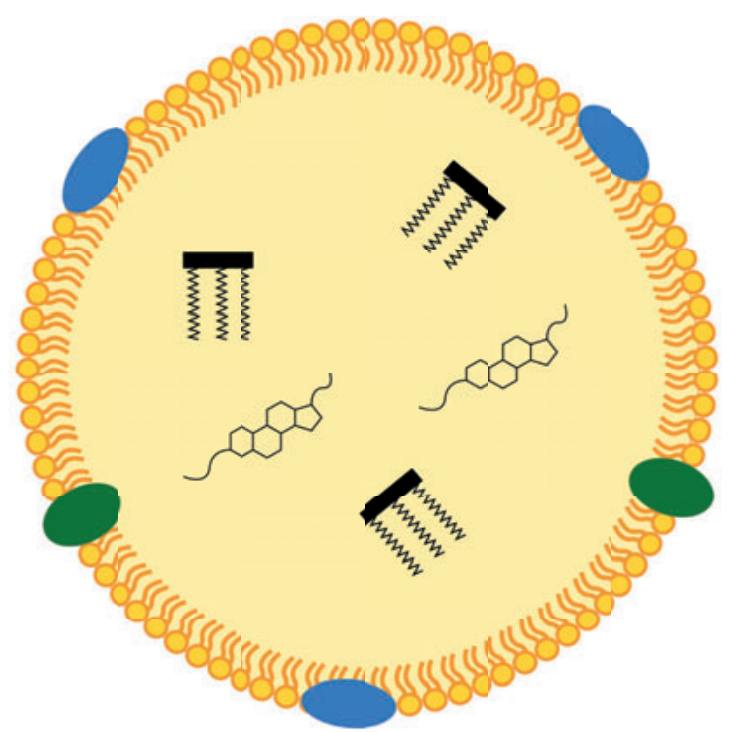

Figure 2. Schematic drawing of a lipid droplet. A lipid droplet is characterized by a phospholipid monolayer which contains several proteins. The core consists mainly of triacylglycerols and cholesterol esters.

\section{Intramyocellular lipids and insulin resistance}

For decades, high levels of IMCL have been associated with insulin resistance (Pan et al., 1997; Krssak et al., 1999; Goodpaster et al., 2000). Indeed, high levels of IMCL are detected in obese and T2DM individuals. However, this relationship disappears when endurance trained athletes are taken into account (Goodpaster et al., 2001). Endurance trained athletes are highly insulin-sensitive while having high amounts of IMCL. This shows that the link between IMCL and insulin sensitivity is unlikely to be causal. Further research has shown that LD morphology (Nielsen et al., 2017; Daemen et al., 2018), LD subcellular distribution (Daemen et al., 2018; Perreault et al., 2018), perilipins on the LDs (Townsend \& McKie, 2017) and lipid species such as ceramide and DAGs (Amati et al., 2011) can play a role in developing insulin resistance. Thus, it is not only important to look at total IMCL levels, but also at factors contributing to the dynamic process of LDs. 


\section{Chapter 1}

\section{Microscopy techniques to study lipid droplets}

To study the factors of LD dynamics, fluorescent microscopy can be used to visualize LDs. Fluorescence microscopy is an imaging technique that make use of fluorophores, such as fluorescent dyes or probes to localize specific proteins to generate an image. A fluorophore is excited and absorbs light with a specific wavelength, and subsequently emits fluorescence (with a higher wavelength and lower energy) which is detected by a detector or a camera.

The use of neutral lipid dyes is a common method to visualize LDs, since LDs store mainly TAGs. In the past, Oil red O (ORO) was a commonly used dye to visualize LDs, originally in bright-field microscopy and at later stages also in widefield fluorescent microscopy (Koopman et al., 2001). However, ORO has its limitations. It requires fresh solutions for each measurement, it needs to be dissolved and filtered and this may result in a lower final concentration and less consistent results (Spangenburg et al., 2011). Moreover, ORO cannot be used for live-cell imaging and due to its broad spectrum, it is difficult to combine with other dyes (Prats et al., 2013). Bodipy 493/503 is another neutral lipid dye and is nowadays preferred over ORO as it is more specific (Prats et al., 2013), can be used for live-cell imaging (Spangenburg et al., 2011) and can be easily combined with other fluorophores or dyes (Prats et al., 2013).

Widefield fluorescent microscopy is a quick, low-cost and conventional method to determine overall lipid content and fibre type distribution in muscle tissue. However, its low xy resolution (>230 nm) makes it difficult to study LDs in detail, such as their size and number and protein coating (Daemen et al., 2016). The diameter of a LD in skeletal muscle typically varies between 100 to $2000 \mathrm{~nm}$ (Digel et al., 2010). Thus, proper imaging requires higher resolution fluorescent microscopy to study these organelles in detail. Laser scanning confocal microscopy is a well-studied method and has a theoretical xy resolution of $140 \mathrm{~nm}$ with a lens of 1.4 numerical aperture (Paddock, 1999). In practice, the xy resolution will be up to $200 \mathrm{~nm}$. Confocal microscopy uses a scanning laser (instead of a lamp in widefield microscopy) and a pinhole to block out-of-focus light. This results in an increased signal-to-noise ratio 
and improved image quality.

In this thesis, Bodipy is combined with immunofluorescence to visualize proteins such as laminin (present in the cell membrane), myosin heavy chain type I (MHC, present in type I muscle fibres) and PLIN5 in human muscle tissue. These are visualized with the use of laser scanning fluorescent confocal microscopy to study the characteristics of LDs within the muscle fibre in detail.

\section{Risk factors and treatment strategies}

Genetic background, family history and socioeconomic status are factors that predispose to overweight, obesity and insulin resistance, but are barely modifiable. Other major contributors that can be more readily modified are amongst others a sedentary lifestyle, unhealthy dietary habits and smoking (Bellou et al., 2018; Zheng et al., 2018). More recently, also ambient temperature (Valdés et al., 2019) and changes in day-night rhythm, including sleep duration (Shan et al., 2015) have been suggested to affect insulin sensitivity. The putative role of LD dynamics in compromised insulin sensitivity prompted us to examine IMCL responses to experimentally induced changes in ambient temperature (cold exposure), day-night rhythm, administration of a nutritional compound known to promote mitochondrial oxidative capacity (resveratrol) and exercising in the fed and fasted state.

\section{Cold exposure}

In insulin-resistant individuals, high plasma TAG levels are observed. High plasma TAG levels may be caused by either blunted inhibition of lipolysis of the white adipose tissue or a high fat diet. High plasma lipid levels facilitate lipid storage in nonadipose tissues, such as the muscle. Abnormal plasma lipid values (dyslipidaemia) increase the risk for the development of cardiovascular complications (Nordestgaard \& Varbo, 2014). Pre-clinical studies showed that brown adipose tissue (BAT) plays an important role in the clearance of TAGs as well (Bartelt et al., 2011). BAT is also present in humans and is activated by cold exposure (Nedergaard et al., 2007; Hanssen et al., 2016). Cold exposure (and BAT activation) has been shown to induce 


\section{Chapter 1}

fat metabolism (Chondronikola et al., 2016; Blondin et al., 2017) and promote insulin sensitivity (Hanssen et al., 2015). If cold exposure can impact postprandial plasma lipid levels in humans needs to be investigated.

\section{Day-night rhythm}

Every cell in our body has a biological clock with a periodicity of about 24 hours and is synchronized by the main clock in our brain. Our biological clock is mainly effected by daylight, but can also be influenced by food intake and exercise (Stenvers et al., 2019). If a person would have a regular day night rhythm, like waking up in the morning, eat regularly and sleeps during the night, one would say that this person's behaviour is aligned with his inner biological clock. Nowadays, however, we live in a 24-hour society with artificial light, irregular eating habits and working during night-time. In this way many persons are misaligned with their inner biological clock. People who work irregular hours, such as shift workers, have indeed a higher risk on developing obesity and insulin resistance (Pan et al., 2011; Gan et al., 2015). Moreover, experimental studies have shown that circadian misalignment with a strictly controlled setting to mimic shift work, leads to reduced glucose tolerance (Scheer et al., 2009; Morris et al., 2016) and compromised muscle insulin sensitivity in humans (Wefers et al., 2018). In mice, a muscle specific knock-out of the core clock gene BMAL1 results in muscle insulin resistance (Dyar et al., 2014). These studies indicate that misalignment of the circadian clock contributes to the impairment of glucose metabolism, mainly through a reduced muscle insulin sensitivity. Others have shown that IMCL and certain lipid species vary across the day-night cycle (Loizides-Mangold et al., 2017; Held et al., 2020). Disruption of the molecular clock results in altered oscillations of lipid species in human myotubes (Loizides-Mangold et al., 2017) and altered lipid metabolism in mice (Dyar et al., 2018). If changes in intramyocellular lipids can explain the circadian misalignment-induced insulin resistance in humans, needs to be examined. 


\section{Resveratrol}

Effective treatment strategies against the development of insulin resistance are exercise and calorie restriction (Balk et al., 2015). Those protocols result in a negative energy balance, hence increasing weight loss, and promotes glucose disposal and mitochondrial function. However, there is a low adherence to these protocols on the long term (Morrato et al., 2007; Middleton et al., 2013; Gibson \& Sainsbury, 2017). For this reason, alternatives strategies are considered that mimic the effects seen by exercise or calorie restriction. Resveratrol is a polyphenol that is naturally present in grapes, berries and peanuts (Cucciolla et al., 2007) and has drawn attention by its positive effects on obesity, glucose handling and insulin resistance in rodents (Szkudelska \& Szkudelski, 2010). Its effects are reported by the activation of SIRT1 and PGC1a (Lagouge et al., 2006) and AMPK (Baur et al., 2006; Timmers et al., 2011). Therefore, resveratrol has been studied as alternative strategy against insulin resistance. In some human trials, resveratrol has shown a positive effect on glucose and lipid handling (Timmers et al., 2011; Bhatt et al., 2012), while other studies did not see an effect (Kjaer et al., 2017; de Ligt et al., 2018). Different doses of resveratrol and different target populations may explain the inconsistency in these results. Interestingly, resveratrol has been seen to induce mitochondrial function (Timmers et al., 2011; Timmers et al., 2016; de Ligt et al., 2018) in combination with increased IMCL (Timmers et al., 2011; Timmers et al., 2016). If this increase in IMCL is a positive effect of resveratrol in lipid handling in humans remains elusive and therefore studying more features of LD dynamics after resveratrol supplementation is warranted.

\section{Exercise}

IMCL can be utilized as energy source during exercise. Long term exercise results in augmented IMCL levels (Schrauwen-Hinderling et al., 2003) and endurance trained athletes have higher levels of IMCL (Daemen et al., 2018). For acute exercise, some studies observed a drop in IMCL content (Stellingwerff et al., 2007; Bucher et al., 2014), while others did not (Kiens \& Richter, 1998). The discrepancy can result from differences in methods used or by the influence of plasma NEFA levels that 


\section{Chapter 1}

function as an energy source or can refuel IMCL stores. Although many studies have investigated the effects of acute exercise on IMCL, more research is needed to study role of nutrition status, or mores specifically plasma NEFA levels, during acute exercise on IMCL levels. Plasma NEFA levels can be enhanced by exercising in the fasted versus the fed state. In addition, not only IMCL levels but also more features of LD dynamics need to be studied after an acute exercise bout and during the recovery of exercise to further elucidate the use of IMCL during exercise.

\section{Outline of the thesis}

The aim of the thesis is to look at the role of LD dynamics in relation to insulin sensitivity in different human intervention studies. In chapter 2, we first reviewed the existing literature on the effects of diet and exercise on LD dynamics in human muscle tissue. To explore the putative role of LD dynamics in insulin resistance we studied in the following chapters lipids and features of LD dynamics after cold exposure, circadian misalignment, resveratrol supplementation and acute exercise. More features of LD dynamics were examined along the chapters. In chapter 3, we analysed how cold exposure in combination with a meal test affects plasma lipid levels, carbohydrate and fat oxidation and muscle mitochondrial function. Next, we wanted to look further into the muscle and examined IMCL and lipid species in relation to compromised insulin sensitivity due to circadian misalignment (chapter 4). In this chapter, we studied LD characteristics in human muscle biopsies from individuals who were either behaviourally aligned with their intrinsic biological clock or misaligned (12 hour swift). Lipidomics was performed to study morning-evening differences and changes in lipid species upon misalignment. In chapter 5, IMCL was further explored with the analysis of the LD protein PLIN5 after an intervention with resveratrol supplementation in metabolically compromised individuals. The aim was to study if resveratrol can remodel IMCL into a healthy lipid profile (i.e. comparable to what has been observed in highly insulin-sensitive individuals). The dynamics of IMCL were more extensively characterized in chapter 6 by studying IMCL and PLIN5 at different time points after an acute exercise protocol. Factors of LD dynamics were 
analysed before exercise, directly post-exercise and $4 \mathrm{~h}$ post-exercise (recovery) under conditions of high or low NEFA levels. In chapter 7, we proposed a microscopy tool to study LD dynamics in vitro. In this chapter the pioneering steps are described to set up this tool in human primary myotubes. In the future, this tool can be used to further explore the differences in LD dynamics between insulin-resistant and insulin-sensitive individuals. Finally, in chapter 8, the overall results of this thesis are discussed in the general discussion. 


\section{References}

Amati F, Dubé JJ, Alvarez-Carnero E, Edreira MM, Chomentowski P, Coen PM, Switzer GE, Bickel PE, Stefanovic-Racic M, Toledo FGS \& Goodpaster BH. (2011). Skeletal Muscle Triglycerides, Diacylglycerols, and Ceramides in Insulin Resistance. Diabetes 60, 2588.

Badin PM, Langin D \& Moro C. (2013). Dynamics of skeletal muscle lipid pools. Trends Endocrinol Metab 24, 607-615.

Balk EM, Earley A, Raman G, Avendano EA, Pittas AG \& Remington PL. (2015). Combined Diet and Physical Activity Promotion Programs to Prevent Type 2 Diabetes Among Persons at Increased Risk: A Systematic Review for the Community Preventive Services Task Force. Ann Intern Med 163, 437-451.

Bartelt A, Bruns OT, Reimer R, Hohenberg H, Ittrich H, Peldschus K, Kaul MG, Tromsdorf UI, Weller H, Waurisch C, Eychmüller A, Gordts PL, Rinninger F, Bruegelmann K, Freund B, Nielsen P, Merkel M \& Heeren J. (2011). Brown adipose tissue activity controls triglyceride clearance. Nat Med 17, 200-205.

Baur JA, Pearson KJ, Price NL, Jamieson HA, Lerin C, Kalra A, Prabhu VV, Allard JS, LopezLluch G, Lewis K, Pistell PJ, Poosala S, Becker KG, Boss O, Gwinn D, Wang M, Ramaswamy S, Fishbein KW, Spencer RG, Lakatta EG, Le Couteur D, Shaw RJ, Navas P, Puigserver P, Ingram DK, de Cabo R \& Sinclair DA. (2006). Resveratrol improves health and survival of mice on a high-calorie diet. Nature 444, 337-342.

Bellou V, Belbasis L, Tzoulaki I \& Evangelou E. (2018). Risk factors for type 2 diabetes mellitus: An exposure-wide umbrella review of meta-analyses. PLoS One 13, e0194127-e0194127.

Bhatt JK, Thomas S \& Nanjan MJ. (2012). Resveratrol supplementation improves glycemic control in type 2 diabetes mellitus. Nutr Res 32, 537-541.

Blondin DP, Tingelstad HC, Noll C, Frisch F, Phoenix S, Guérin B, Turcotte ÉE, Richard D, Haman F \& Carpentier AC. (2017). Dietary fatty acid metabolism of brown adipose tissue in cold-acclimated men. Nature Communications 8, 14146.

Bosma M, Minnaard R, Sparks LM, Schaart G, Losen M, de Baets MH, Duimel H, Kersten S, Bickel PE, Schrauwen P \& Hesselink MKC. (2012). The lipid droplet coat protein perilipin 5 also localizes to muscle mitochondria. Histochemistry and Cell Biology 137, 205-216.

Bosma M, Sparks LM, Hooiveld GJ, Jorgensen JA, Houten SM, Schrauwen P, Kersten S \& Hesselink MK. (2013). Overexpression of PLIN5 in skeletal muscle promotes oxidative gene expression and intramyocellular lipid content without compromising insulin sensitivity. Biochim Biophys Acta 1831, 844-852.

Bucher J, Krusi M, Zueger T, Ith M, Stettler C, Diem P, Boesch C, Kreis R \& Christ E. (2014). The effect of a single $2 \mathrm{~h}$ bout of aerobic exercise on ectopic lipids in skeletal muscle, liver and the myocardium. Diabetologia 57, 1001-1005.

Cersosimo E, Triplitt C, Solis-Herrera C, Mandarino LJ \& DeFronzo RA. (2000). Pathogenesis of Type 2 Diabetes Mellitus. In Endotext, ed. Feingold KR, Anawalt B, Boyce A, Chrousos G, de Herder WW, Dungan K, Grossman A, Hershman JM, Hofland HJ, Kaltsas G, Koch C, Kopp P, Korbonits M, McLachlan R, Morley JE, New M, Purnell J, Singer F, Stratakis CA, Trence DL \& Wilson DP. MDText.com, Inc. Copyright (C) 2000-2020, MDText.com, Inc., South Dartmouth (MA). 
Chondronikola M, Volpi E, Børsheim E, Porter C, Saraf MK, Annamalai P, Yfanti C, Chao T, Wong D, Shinoda K, Labbė SM, Hurren NM, Cesani F, Kajimura S \& Sidossis LS. (2016). Brown Adipose Tissue Activation Is Linked to Distinct Systemic Effects on Lipid Metabolism in Humans. Cell Metab 23, 1200-1206.

Cucciolla V, Borriello A, Oliva A, Galletti P, Zappia V \& Della Ragione F. (2007). Resveratrol: from basic science to the clinic. Cell Cycle 6, 2495-2510.

Daemen S, Gemmink A, Brouwers B, Meex RCR, Huntjens PR, Schaart G, Moonen-Komips E, Jorgensen J, Hoeks J, Schrauwen P \& Hesselink MKC. (2018). Distinct lipid droplet characteristics and distribution unmask the apparent contradiction of the athlete's paradox. Molecular metabolism 17, 71-81.

Daemen S, van Zandvoort M, Parekh SH \& Hesselink MKC. (2016). Microscopy tools for the investigation of intracellular lipid storage and dynamics. Molecular metabolism 5, 153-163.

de Ligt M, Bruls YMH, Hansen J, Habets MF, Havekes B, Nascimento EBM, Moonen-Kornips E, Schaart G, Schrauwen-Hinderling VB, van Marken Lichtenbelt W \& Schrauwen P. (2018). Resveratrol improves ex vivo mitochondrial function but does not affect insulin sensitivity or brown adipose tissue in first degree relatives of patients with type 2 diabetes. Molecular metabolism 12, 39-47.

DeFronzo RA \& Tripathy D. (2009). Skeletal Muscle Insulin Resistance Is the Primary Defect in Type 2 Diabetes. Diabetes Care 32, S157.

Deshpande AD, Harris-Hayes M \& Schootman M. (2008). Epidemiology of diabetes and diabetes-related complications. Physical therapy 88, 1254-1264.

Digel M, Ehehalt R \& Füllekrug J. (2010). Lipid droplets lighting up: Insights from live microscopy. FEBS Letters 584, 2168-2175.

Dyar KA, Ciciliot S, Wright LE, Biensø RS, Tagliazucchi GM, Patel VR, Forcato M, Paz MIP, Gudiksen A, Solagna F, Albiero M, Moretti I, Eckel-Mahan KL, Baldi P, SassoneCorsi P, Rizzuto R, Bicciato S, Pilegaard H, Blaauw B \& Schiaffino S. (2014). Muscle insulin sensitivity and glucose metabolism are controlled by the intrinsic muscle clock. Molecular metabolism 3, 29-41.

Dyar KA, Hubert MJ, Mir AA, Ciciliot S, Lutter D, Greulich F, Quagliarini F, Kleinert M, Fischer K, Eichmann TO, Wright LE, Peña Paz MI, Casarin A, Pertegato V, Romanello V, Albiero M, Mazzucco S, Rizzuto R, Salviati L, Biolo G, Blaauw B, Schiaffino S \& Uhlenhaut NH. (2018). Transcriptional programming of lipid and amino acid metabolism by the skeletal muscle circadian clock. PLOS Biology 16, e2005886.

Essén B, Jansson E, Henriksson J, Taylor AW \& Saltin B. (1975). Metabolic Characteristics of Fibre Types in Human Skeletal Muscle. Acta Physiologica Scandinavica 95, 153-165.

Farese RV, Jr. \& Walther TC. (2009). Lipid droplets finally get a little R-E-S-P-E-C-T. Cell 139, 855-860.

Gan Y, Yang C, Tong X, Sun H, Cong Y, Yin X, Li L, Cao S, Dong X, Gong Y, Shi O, Deng J, Bi $\mathrm{H} \& \mathrm{Lu} Z$. (2015). Shift work and diabetes mellitus: a meta-analysis of observational studies. Occupational and Environmental Medicine 72, 72.

Gemmink A, Bosma M, Kuijpers HJ, Hoeks J, Schaart G, van Zandvoort MA, Schrauwen P \& Hesselink MK. (2016). Decoration of intramyocellular lipid droplets with PLIN5 modulates fasting-induced insulin resistance and lipotoxicity in humans. Diabetologia 59, 1040-1048.

Gemmink A, Daemen S, Kuijpers HJH, Schaart G, Duimel H, López-Iglesias C, van Zandvoort 


\section{Chapter 1}

MAMJ, Knoops K \& Hesselink MKC. (2018). Super-resolution microscopy localizes perilipin 5 at lipid droplet-mitochondria interaction sites and at lipid droplets juxtaposing to perilipin 2. Biochimica et Biophysica Acta (BBA) - Molecular and Cell Biology of Lipids 1863, 1423-1432.

Gibson AA \& Sainsbury A. (2017). Strategies to Improve Adherence to Dietary Weight Loss Interventions in Research and Real-World Settings. Behavioral sciences (Basel, Switzerland) 7.

Goodpaster BH, He J, Watkins S \& Kelley DE. (2001). Skeletal muscle lipid content and insulin resistance: evidence for a paradox in endurance-trained athletes. J Clin Endocrinol Metab 86, 5755-5761.

Goodpaster BH, Theriault R, Watkins SC \& Kelley DE. (2000). Intramuscular lipid content is increased in obesity and decreased by weight loss. Metabolism 49, 467-472.

Hanssen MJW, Hoeks J, Brans B, van der Lans AAJJ, Schaart G, van den Driessche JJ, Jörgensen JA, Boekschoten MV, Hesselink MKC, Havekes B, Kersten S, Mottaghy FM, van Marken Lichtenbelt WD \& Schrauwen P. (2015). Short-term cold acclimation improves insulin sensitivity in patients with type 2 diabetes mellitus. Nature Medicine 21, 863865.

Hanssen MJW, van der Lans AAJJ, Brans B, Hoeks J, Jardon KMC, Schaart G, Mottaghy FM, Schrauwen P \& van Marken Lichtenbelt WD. (2016). Short-term Cold Acclimation Recruits Brown Adipose Tissue in Obese Humans. Diabetes 65, 1179.

Held NM, Wefers J, van Weeghel M, Daemen S, Hansen J, Vaz FM, van Moorsel D, Hesselink MKC, Houtkooper RH \& Schrauwen P. (2020). Skeletal muscle in healthy humans exhibits a day-night rhythm in lipid metabolism. Molecular metabolism 37, 100989.

Itani SI, Ruderman NB, Schmieder F \& Boden G. (2002). Lipid-Induced Insulin Resistance in Human Muscle Is Associated With Changes in Diacylglycerol, Protein Kinase C, and I $\kappa$ B- $\alpha$. Diabetes 51, 2005-2011.

Kiens B \& Richter EA. (1998). Utilization of skeletal muscle triacylglycerol during postexercise recovery in humans. Am J Physiol 275, E332-337.

Kjaer TN, Ornstrup MJ, Poulsen MM, Stodkilde-Jorgensen H, Jessen N, Jorgensen JOL, Richelsen B \& Pedersen SB. (2017). No Beneficial Effects of Resveratrol on the Metabolic Syndrome: A Randomized Placebo-Controlled Clinical Trial. J Clin Endocr Metab 102, 1642-1651.

Koopman R, Schaart G \& Hesselink MK. (2001). Optimisation of oil red O staining permits combination with immunofluorescence and automated quantification of lipids. Histochem Cell Biol 116, 63-68.

Krssak M, Falk Petersen K, Dresner A, DiPietro L, Vogel SM, Rothman DL, Roden M \& Shulman GI. (1999). Intramyocellular lipid concentrations are correlated with insulin sensitivity in humans: a ${ }^{1} \mathrm{H}$ NMR spectroscopy study. Diabetologia 42, 113-116.

Laaksonen MA, Knekt P, Rissanen H, Härkänen T, Virtala E, Marniemi J, Aromaa A, Heliövaara M \& Reunanen A. (2010). The relative importance of modifiable potential risk factors of type 2 diabetes: a meta-analysis of two cohorts. European Journal of Epidemiology 25, 115-124.

Lagouge M, Argmann C, Gerhart-Hines Z, Meziane H, Lerin C, Daussin F, Messadeq N, Milne J, Lambert P, Elliott P, Geny B, Laakso M, Puigserver P \& Auwerx J. (2006). Resveratrol improves mitochondrial function and protects against metabolic disease by activating SIRT1 and PGC-1alpha. Cell 127, 1109-1122. 
Listenberger LL, Han X, Lewis SE, Cases S, Farese RV, Ory DS \& Schaffer JE. (2003). Triglyceride accumulation protects against fatty acid-induced lipotoxicity. Proceedings of the National Academy of Sciences 100, 3077.

Liu L, Zhang Y, Chen N, Shi X, Tsang B \& Yu YH. (2007). Upregulation of myocellular DGAT1 augments triglyceride synthesis in skeletal muscle and protects against fat-induced insulin resistance. J Clin Invest 117, 1679-1689.

Loizides-Mangold U, Perrin L, Vandereycken B, Betts JA, Walhin J-P, Templeman I, Chanon S, Weger BD, Durand C, Robert M, Paz Montoya J, Moniatte M, Karagounis LG, Johnston JD, Gachon F, Lefai E, Riezman H \& Dibner C. (2017). Lipidomics reveals diurnal lipid oscillations in human skeletal muscle persisting in cellular myotubes cultured in vitro. Proceedings of the National Academy of Sciences 114, E8565-E8574.

Loon LJCv, Koopman R, Manders R, Weegen Wvd, Kranenburg GPv \& Keizer HA. (2004). Intramyocellular lipid content in type 2 diabetes patients compared with overweight sedentary men and highly trained endurance athletes. 287, E558-E565.

Middleton KR, Anton SD \& Perri MG. (2013). Long-Term Adherence to Health Behavior Change. American journal of lifestyle medicine 7, 395-404.

Morrato EH, Hill JO, Wyatt HR, Ghushchyan V \& Sullivan PW. (2007). Physical Activity in U.S. Adults With Diabetes and At Risk for Developing Diabetes, 2003. Diabetes Care 30, 203.

Morris CJ, Purvis TE, Mistretta J \& Scheer FAJL. (2016). Effects of the Internal Circadian System and Circadian Misalignment on Glucose Tolerance in Chronic Shift Workers. The Journal of Clinical Endocrinology \& Metabolism 101, 1066-1074.

Nedergaard J, Bengtsson T \& Cannon B. (2007). Unexpected evidence for active brown adipose tissue in adult humans. American Journal of Physiology-Endocrinology and Metabolism 293, E444-E452.

Nguyen TB, Louie SM, Daniele JR, Tran Q, Dillin A, Zoncu R, Nomura DK \& Olzmann JA. (2017). DGAT1-Dependent Lipid Droplet Biogenesis Protects Mitochondrial Function during Starvation-Induced Autophagy. Dev Cell 42, 9-21.e25.

Nielsen J, Christensen AE, Nellemann B \& Christensen B. (2017). Lipid droplet size and location in human skeletal muscle fibers are associated with insulin sensitivity. Am J Physiol Endocrinol Metab 313, E721-E730.

Nordestgaard BG \& Varbo A. (2014). Triglycerides and cardiovascular disease. Lancet 384, 626-635.

Paddock SW. (1999). Confocal Laser Scanning Microscopy. BioTechniques 27, 992-1004.

Pan A, Schernhammer ES, Sun Q \& Hu FB. (2011). Rotating Night Shift Work and Risk of Type 2 Diabetes: Two Prospective Cohort Studies in Women. PLOS Medicine 8, e1001141.

Pan DA, Lillioja S, Kriketos AD, Milner MR, Baur LA, Bogardus C, Jenkins AB \& Storlien LH. (1997). Skeletal muscle triglyceride levels are inversely related to insulin action. Diabetes 46, 983-988.

Park T-S, Hu Y, Noh H-L, Drosatos K, Okajima K, Buchanan J, Tuinei J, Homma S, Jiang X-C, Abel ED \& Goldberg IJ. (2008). Ceramide is a cardiotoxin in lipotoxic cardiomyopathy. J Lipid Res 49, 2101-2112.

Perreault L, Newsom SA, Strauss A, Kerege A, Kahn DE, Harrison KA, Snell-Bergeon JK, Nemkov T, D'Alessandro A, Jackman MR, MacLean PS \& Bergman BC. (2018). Intracellular localization of diacylglycerols and sphingolipids influences insulin sensitivity and mitochondrial function in human skeletal muscle. JCI Insight 3. 


\section{Chapter 1}

Petersen MC \& Shulman GI. (2018). Mechanisms of Insulin Action and Insulin Resistance. Physiol Rev 98, 2133-2223.

Prats C, Gomez-Cabello A, Nordby P, Andersen JL, Helge JW, Dela F, Baba O \& Ploug T. (2013). An optimized histochemical method to assess skeletal muscle glycogen and lipid stores reveals two metabolically distinct populations of type I muscle fibers. PLoS One 8, e77774-e77774.

Rambold Angelika S, Cohen S \& Lippincott-Schwartz J. (2015). Fatty Acid Trafficking in Starved Cells: Regulation by Lipid Droplet Lipolysis, Autophagy, and Mitochondrial Fusion Dynamics. Developmental Cell 32, 678-692.

Scheer FAJL, Hilton MF, Mantzoros CS \& Shea SA. (2009). Adverse metabolic and cardiovascular consequences of circadian misalignment. Proceedings of the National Academy of Sciences 106, 4453.

Schnurr TM, Jakupović H, Carrasquilla GD, Ängquist L, Grarup N, Sørensen TIA, Tjønneland A, Overvad K, Pedersen O, Hansen T \& Kilpeläinen TO. (2020). Obesity, unfavourable lifestyle and genetic risk of type 2 diabetes: a case-cohort study. Diabetologia 63, 1324-1332.

Schrauwen-Hinderling VB, Hesselink MKC, Schrauwen P \& Kooi ME. (2006). Intramyocellular Lipid Content in Human Skeletal Muscle. 14, 357-367.

Schrauwen-Hinderling VB, Schrauwen P, Hesselink MK, van Engelshoven JM, Nicolay K, Saris WH, Kessels AG \& Kooi ME. (2003). The increase in intramyocellular lipid content is a very early response to training. J Clin Endocrinol Metab 88, 1610-1616.

Shan Z, Ma H, Xie M, Yan P, Guo Y, Bao W, Rong Y, Jackson CL, Hu FB \& Liu L. (2015). Sleep Duration and Risk of Type 2 Diabetes: A Meta-analysis of Prospective Studies. Diabetes Care 38, 529.

Shepherd SO, Strauss JA, Wang Q, Dube JJ, Goodpaster B, Mashek DG \& Chow LS. (2017). Training alters the distribution of perilipin proteins in muscle following acute free fatty acid exposure. J Physiol 595, 5587-5601.

Spangenburg EE, Pratt SJP, Wohlers LM \& Lovering RM. (2011). Use of BODIPY (493/503) to visualize intramuscular lipid droplets in skeletal muscle. J Biomed Biotechnol 2011, 598358-598358.

Stellingwerff T, Boon H, Jonkers RA, Senden JM, Spriet LL, Koopman R \& van Loon LJ. (2007). Significant intramyocellular lipid use during prolonged cycling in endurance-trained males as assessed by three different methodologies. Am J Physiol Endocrinol Metab 292, E1715-1723.

Stenvers DJ, Scheer FAJL, Schrauwen P, la Fleur SE \& Kalsbeek A. (2019). Circadian clocks and insulin resistance. Nature Reviews Endocrinology 15, 75-89.

Szendroedi J, Yoshimura T, Phielix E, Koliaki C, Marcucci M, Zhang D, Jelenik T, Müller J, Herder C, Nowotny P, Shulman GI \& Roden M. (2014). Role of diacylglycerol activation of PKC $\theta$ in lipid-induced muscle insulin resistance in humans. Proceedings of the National Academy of Sciences 111, 9597-9602.

Szkudelska K \& Szkudelski T. (2010). Resveratrol, obesity and diabetes. Eur J Pharmacol 635, 1-8.

Timmers S, de Ligt M, Phielix E, van de Weijer T, Hansen J, Moonen-Kornips E, Schaart G, Kunz I, Hesselink MK, Schrauwen-Hinderling VB \& Schrauwen P. (2016). Resveratrol as Add-on Therapy in Subjects With Well-Controlled Type 2 Diabetes: A Randomized Controlled Trial. Diabetes Care 39, 2211-2217. 
Timmers S, Konings E, Bilet L, Houtkooper RH, van de Weijer T, Goossens GH, Hoeks J, van der Krieken S, Ryu D, Kersten S, Moonen-Kornips E, Hesselink MKC, Kunz I, Schrauwen-Hinderling VB, Blaak E, Auwerx J \& Schrauwen P. (2011). Calorie restriction-like effects of 30 days of resveratrol supplementation on energy metabolism and metabolic profile in obese humans. Cell Metab 14, 612-622.

Townsend LK \& McKie GL. (2017). Using PLIN proteins to explain the athlete's paradox. J Physiol 595, 6819-6820.

Valdés S, Doulatram-Gamgaram V, Lago A, Torres FG, Badía-Guillén R, Olveira G, Goday A, Calle-Pascual A, Castaño L, Castell C, Delgado E, Menendez E, Franch-Nadal J, Gaztambide S, Girbés J, Gomis R, Ortega E, Galán-García JL, Aguilera-Venegas G, Soriguer F \& Rojo-Martínez G. (2019). Ambient temperature and prevalence of diabetes and insulin resistance in the Spanish population: Di@bet.es study. European Journal of Endocrinology 180, 273.

Vazquez G, Duval S, Jacobs DR, Jr. \& Silventoinen K. (2007). Comparison of Body Mass Index, Waist Circumference, and Waist/Hip Ratio in Predicting Incident Diabetes: A MetaAnalysis. Epidemiologic Reviews 29, 115-128.

Verma MK, Yateesh AN, Smitha R, Neelima K, Pallavi PM, Reddy M, Poornima J, Oommen AM, Jagannath MR \& Somesh BP. (2014). Integrated analysis of chronic lipotoxicity on muscle metabolism and stress and its reversal by antioxidants. SpringerPlus 3, 251.

Volksgezondheidenzorg.info. (2020a). Overgewicht Nederlandse bevolking. RIVM, Bilthoven. Volksgezondheidenzorg.info. (2020b). Jaarprevelentie diabetes mellitus 2019. RIVM, Bilthoven. Volksgezondheidenzorg.info. (2020c). Ranglijst aandoeningen op basis van ziektelast (in DALY's). RIVM, Bilthoven.

Wefers J, van Moorsel D, Hansen J, Connell NJ, Havekes B, Hoeks J, van Marken Lichtenbelt WD, Duez H, Phielix E, Kalsbeek A, Boekschoten MV, Hooiveld GJ, Hesselink MKC, Kersten S, Staels B, Scheer F \& Schrauwen P. (2018). Circadian misalignment induces fatty acid metabolism gene profiles and compromises insulin sensitivity in human skeletal muscle. Proc Natl Acad Sci U S A 115, 7789-7794.

Zheng Y, Ley SH \& Hu FB. (2018). Global aetiology and epidemiology of type 2 diabetes mellitus and its complications. Nature Reviews Endocrinology 14, 88-98. 


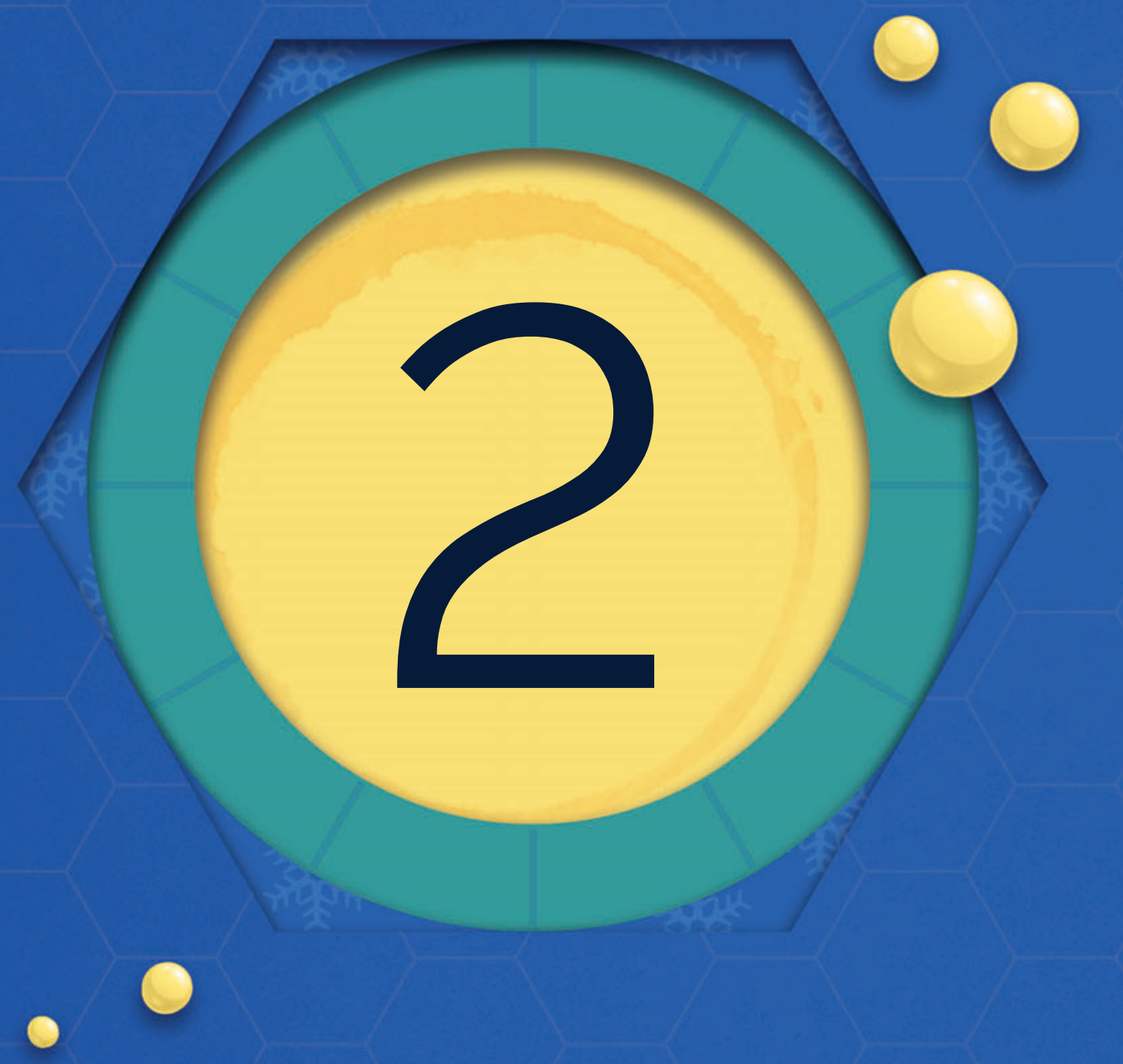




\title{
The effect of diet and exercise on lipid droplet dynamics in human muscle
}

\author{
tissue
}

Sabine Daemen, Nynke van Polanen, Matthijs K.C. Hesselink

Published in Journal of Experimental Biology, 2018; Mar 7; 221 (Pt Suppl 1) 


\section{Abstract}

The majority of fat in the human body is stored as triacylglycerols in white adipose tissue. In the obese state, adipose tissue mass expands and excess lipids are stored in non-adipose tissues, such as skeletal muscle. Lipids are stored in skeletal muscle in the form of small lipid droplets. Although originally viewed as dull organelles that simply store lipids as a consequence of lipid overflow from adipose tissue, lipid droplets are now recognized as key components in the cell that exert a variety of relevant functions in multiple tissues (including muscle). Here, we review the effect of diet and exercise interventions on myocellular lipid droplets and their putative role in insulin sensitivity from a human perspective. We also provide an overview of lipid droplet biology and identify gaps for future research. 


\section{Introduction}

Dietary fatty acids are stored in adipose tissue. However, excess lipid intake leads to hyperlipidaemia and elevated plasma levels of triacylglycerol (TAG) and free fatty acids (FFAs). The so-called overflow of lipids promotes the storage of excess fat in non-adipose tissues such as skeletal muscle (van Hees et al., 2010). This phenomenon is referred to as ectopic fat storage. The lipid overflow hypothesis suggests that ectopic fat storage is merely a way of sequestering excess lipids. Thus, excess lipids upon lipid overflow augment total intramyocellular lipid (IMCL) content. IMCL is dispersed throughout the muscle in small lipid droplets (LDs). In the past, LDs were viewed as inert storage sites; however, they are now considered to be active organelles (Krahmer et al., 2009).

Ectopic fat storage has been suggested to interfere with normal cell function, a phenomenon referred to as lipotoxicity (Unger, 2003). Skeletal muscle is an important organ in the storage and release of fatty acids upon changes in supply and demand and, hence, is prone to lipotoxicity. Human skeletal muscle is responsible for approximately $80 \%$ of post-prandial glucose uptake (under healthy conditions), which is impeded in muscles with excess IMCL (Blaak, 2004). It is therefore interesting to note that obese individuals or patients with type 2 diabetes have elevated IMCL content compared with lean individuals (Goodpaster et al., 2001) and that IMCL is also elevated in insulin-resistant offspring of patients with type 2 diabetes (Jacob et al., 1999; Petersen et al., 2004). This supports the notion that excess IMCL can be an early event in type 2 diabetes development. Multiple papers have reported negative correlations of IMCL content with insulin sensitivity (Pan et al., 1997; Jacob et al., 1999; Krssak et al., 1999; Goodpaster et al., 2001; Anderwald et al., 2002). However, this correlation is absent in trained athletes (Goodpaster et al., 2001; Thamer et al., 2003), a phenomenon referred to as the 'athlete's paradox' (Goodpaster et al., 2001); despite high levels of IMCL, trained athletes remain highly insulin sensitive. The mechanistic link between IMCL content and muscle insulin sensitivity, however, remains elusive. It has been suggested that the capacity to readily shuttle excess fatty acids to inert storage in LDs in the form of TAG along with an adjustment of TAG hydrolysis and 
fatty acid oxidation, ameliorates the insulin desensitizing effects of lipids (Badin et al., 2013). Jointly, these processes are referred to as LD dynamics (Badin et al., 2013).

The dynamic nature of LDs seems to depend on a variety of factors, including nutritional and dietary factors, as well as acute physical exercise and exercise training (Bosma et al., 2012a). Multiple characteristics of LDs have been linked to the dynamic nature of LDs (Figure 1) and, hence, are suspected to contribute somehow to the intricate relationship between ectopic fat storage and insulin sensitivity. Likewise, the size, number, subcellular distribution, composition and protein coating of LDs, and the interaction of LDs with other subcellular organelles, such as mitochondria and sarcoplasmic reticulum, have all been hypothesized to be determinants of myocellular insulin sensitivity.

Here, we will briefly review basic concepts of LD biology in human skeletal muscle, specifically in relation to insulin sensitivity, with the aim of providing a fundamental understanding of how dietary interventions and physical exercise affect LD dynamics.
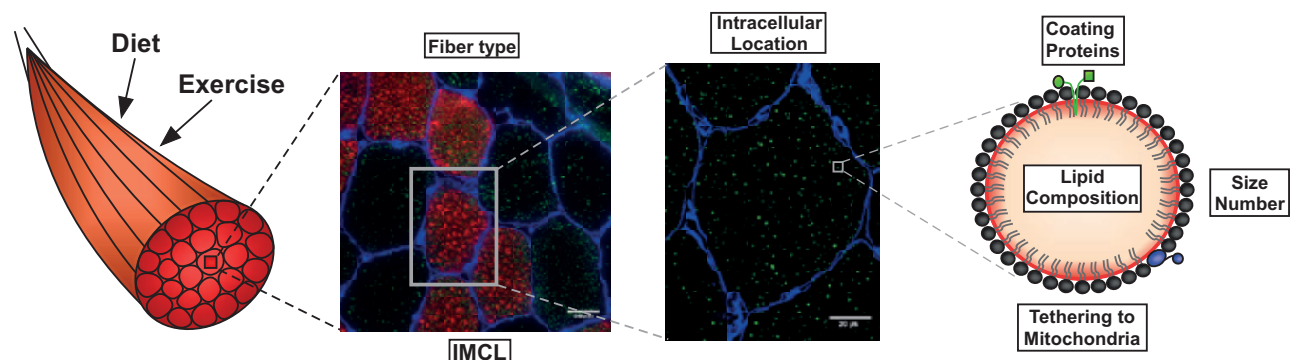

Figure 1. Schematic overview of the characteristics of muscle lipid droplet (LD) dynamics addressed in this review. Intramyocellular lipid (IMCL) storage occurs in LDs, which are considered to be highly dynamic organelles with an important role in regulating cellular metabolic health. Multiple dynamic characteristics of $L D$ s, such as cellular localization (muscle fibre type specificity as well as subcellular localization), $L D$ morphology ( $L D$ size and number), interactions of $L D$ s with mitochondria and with proteins regulating $L D$ turnover (e.g. PAT protein family, lipolytic enzyme adipose triglyceride lipase and its cofactors) and the composition of lipids in the LD have been linked to (patho)physiology, such as insulin sensitivity. Diet and exercise interventions affect the dynamics of the LDs. The widefield microscopy image (left-hand image) and the confocal microscopy image (right-hand image) are cross-sectional views of a cryosection of a biopsy of the vastus lateralis muscle and show human muscle fibres; the basement membrane protein laminin is shown in blue (secondary antibody labelled with Alexa Fluor 405), LDs are stained green (Bodipy 493/503) and type I fibres are shown in red (primary antibody against myosin heavy chain type 1; secondary antibody labelled with Alexa Fluor 555). 


\section{Lipid droplet structure, biogenesis and growth}

LDs in skeletal muscle are relatively small $\left(0.20-0.50 \mu \mathrm{m}^{2}\right)$ and typically comprise a neutral lipid core composed of lipid esters, including TAGs and, to a lesser extent, cholesterol esters (Walther \& Farese, 2012). Usually, myocellular LDs are surrounded by a phospholipid monolayer that provides a base to a variety of proteins involved in storage and release of fatty acids from LDs (Walther \& Farese, 2012). Exactly how LDs are formed has not been elucidated. A variety of models for LD formation have been hypothesized (Wanner et al., 1981; Murphy \& Vance, 1999; Zweytick et al., 2000; Waltermann et al., 2005; Ploegh, 2007; Walther \& Farese, 2009). The classical model

describes the 'budding off' theory and suggests that globular structures of lipid esters within the endoplasmic reticulum (ER) are budded off, hence resulting in a LD coated with a phospholipid monolayer (Murphy \& Vance, 1999). Another model that has gained momentum over the years involves the accumulation of lipids in membranes of small ER vesicles, resulting in LD formation and release (Walther \& Farese, 2009). Although these models are not mutually exclusive, none of the models is underpinned by thorough and complete experimental proof.

Even within a single cell type, such as skeletal muscle, LDs can vary substantially in size under a variety of conditions. How LDs change in size is not completely understood. However, three models are currently under consideration (Thiam et al., 2013). (i) The fusion of LDs via pores in the phospholipid monolayer of two LDs results in an exchange of lipids and the fusion of LDs. (ii) An exchange of molecules from one LD to another in a process referred to as ripening, which results in a linear increase in LD size over time. Typically, this process occurs alongside increases in the phospholipids of the LD monolayer. In this model, some LDs grow at the expense of others that shrink, resulting typically in a wider range in LD size. (iii) The expansion of LDs by the cellular uptake of excess fatty acids from the circulation and the incorporation of these fatty acids in the LD as TAG. Given that each of these three processes distinctly affects the lipid interface, it is not unlikely that the type of process responsible for LD growth also affects the physiological properties of large LDs. 


\section{Lipid droplet coat proteins of the perilipin family}

The TAG molecules making up the LDs in skeletal muscle are subject to enduring cycles of hydrolysis and resynthesis. This dynamic nature is tightly orchestrated by a series of proteins possessing lipolytic activity (e.g. the main TAG lipase, adipose triglyceride lipase (ATGL) (Zimmermann et al., 2004) whose activity is regulated by stimulatory and inhibitory co-factors). Regulation of LD synthesis and degradation is also under partial control of a set of structurally alike proteins from the perilipin family, also known as PAT proteins (Kimmel et al., 2010). Of the PAT proteins currently known, PLIN2 (Brasaemle et al., 1997) and PLIN5 (Wolins et al., 2006; Yamaguchi et al., 2006; Dalen et al., 2007) appear most important in skeletal muscle. PLIN2 dissociates the lipase from the LD and, hence, prevents lipolysis from occurring (Listenberger et al., 2007). PLIN5 is present at the LD surface, particularly at LDs neighbouring mitochondria as well as at LD-mitochondrial contact sites (Bosma et al., 2012b) and is supposed to regulate LD lipolysis in conjunction with ATGL and its co-factors. More specifically, PLIN5 has been suggested to be involved in fine-tuning the release of fatty acids from LD lipolysis to the mitochondrial oxidative demand. In general, the emerging picture is that in the absence of PLINs, muscle becomes devoid of LDs (Bosma et al., 2012a) due to ongoing lipolysis. Increases in any of these PLINs results in large LDs and high IMCL content (Bosma et al., 2012b). Thus, proteins of the PAT family are probable candidates to affect the dynamic nature of LDs, resulting in morphological changes such as altered LD number and size and, possibly, lipid composition and interorganelle interaction. 


\section{Lipid droplet turnover}

Myocellular LDs provide the main lipid reservoir to provide energy by hydrolysis of the TAG they harbour and provide building blocks for membrane synthesis (Farese \& Walther, 2009). To fulfil these roles, esterification of fatty acids into TAG and subsequent storage in LDs is essential. It has even been argued that plasma fatty acids are first incorporated in LDs prior to use for oxidation during exercise (Kanaley et al., 2009). Thus, LDs should be highly dynamic and be capable of readily fine-tuning to changes in the supply and demand of fatty acids.

Indeed, LDs making up the IMCL pool can exhibit a high turnover during both rest (Sacchetti et al., 2004; Kanaley et al., 2009) and exercise (Guo et al., 2000), whereas total IMCL content remains unaffected (Guo et al., 2000; Sacchetti et al., 2004). In athletes, the synthesis rate of IMCL is higher than in untrained individuals (Bergman et al., 2010). By contrast, lower rates of IMCL synthesis and oxidation have been reported in obese and pre-diabetic males with observations in obese and pre-diabetic males (Perreault et al., 2010) and in patients with type 2 diabetes (Blaak \& Wagenmakers, 2002) than in healthy males. Thus, the IMCL pool in trained athletes represents a highly dynamic pool, whereas the IMCL pool in patients with type 2 diabetes has lost its dynamic character.

A highly dynamic LD pool relies on readily and efficiently sequestering of fatty acids in LDs and well-controlled hydrolysis of TAG in the LD to fuel fat oxidation in a timely and adequate fashion. Studies using cell and rodent models have revealed that the absence of PLIN2 (Bosma et al., 2012a) or PLIN5 (Mason et al., 2014) results in a marked reduction in LD number and size. However, under these conditions, the most likely explanation for the loss of LDs is not the compromised incorporation of fatty acids in TAG, but rather uncontrolled and/or continuous lipolysis. Data from human studies on sequestering and release of fatty acids from LDs is, however, largely lacking.

Rare, genetic disorders resulting in compromised ATGL activity (Fischer et al., 2007), either directly by affecting ATGL protein content or function, or by reducing the 


\section{Chapter 2}

function of its co-factor comparative gene identification 58 (CGI-58; also known as $\alpha-\beta$ hydrolase domain-containing protein 5, ABHD5) (Lass et al., 2006; Schweiger et al., 2008; Schweiger et al., 2009), profoundly compromise LD dynamics and result in severe metabolic aberrations. Following the reasoning that inert storage of fatty acids in LDs does not necessarily impede cell function, the severity of the metabolic aberrations observed in patients with ATGL or CGI-58 mutations is somewhat surprising. Studies in rodents have revealed that mice lacking ATGL had severely compromised mitochondrial function and consequently possessed low fat oxidative capacity (Haemmerle et al., 2006). Given that fatty acids are ligands for peroxisome proliferator activated receptor (PPAR)-mediated gene expression, it has been hypothesized that in the absence of LD hydrolysis, the limited availability of fatty acids for PPAR activation may be the underlying factor for the compromised oxidative gene expression observed when ATGL function is limited (Haemmerle et al., 2011). This notion was substantiated by the observation that administration of synthetic PPAR ligands to mice (Haemmerle et al., 2011) and ATGL mutant humans (van de Weijer et al., 2013) promoted the oxidative phenotype, without major effects on LD number or size or TAG hydrolysis (Haemmerle et al., 2011; van de Weijer et al., 2013). These observations indicate that the maintenance of TAG hydrolysis capacity is an essential aspect of LD dynamics, and links proper LD dynamics to proper cell function, including the maintenance of an oxidative phenotype (see also the 'Role of oxidative capacity and mitochondria' section).

\section{Role of oxidative capacity and mitochondria}

IMCL is an important substrate during exercise in endurance-trained athletes (Stellingwerff et al., 2007). In athletes, a high IMCL level coexists with a high oxidative capacity and a high contribution of lipids to total energy provision. This is in contrast with sedentary subjects, where lipid supply often exceeds the oxidative demand or capacity. In athletes, the increased demand may result in high lipid (droplet) turnover, which may affect important parameters such as levels of specific lipid species, cellular localization of lipids, LD protein coating and LD-organelle 
interactions. Moreover, mitochondrial dysfunction has been implicated in insulin resistance in type 2 diabetes, further compromising the ability to oxidize fat. Thus, mitochondrial deficits may lead to incomplete $\beta$-oxidation, resulting in accumulation of lipid intermediates such as diacylglycerol (DAGs), ceramides or other toxic lipid species (see also the 'Lipid composition and intermediates' section).

Maintaining a tight match between lipid supply and lipid usage appears to be important to prevent lipotoxicity. Therefore, a tight tethering of LDs and mitochondria could be key for effectively shuttling fatty acids into mitochondria. Indeed, LDs and mitochondria show a close spatial approximation (Hoppeler, 1999; Shaw et al., 2008). PLIN5 is specifically located at the LD-mitochondrial interface (Bosma et al., 2012b), which suggests that this protein has a role in shuttling fatty acids from LD to mitochondria. The exact nature of LD-mitochondrial interactions remains elusive.

Nowadays, mitochondria are no longer viewed as single organelles, but they can form an organized network, which can be observed both in cultured muscle cells and muscle tissue. Just like LDs, the mitochondrial network is also thought to be highly dynamic and can adapt to cellular energy status via the fission and fusion of mitochondria. Interestingly, LDs appear to be in conjunction with the mitochondrial reticular network (Figure 2). A properly fused mitochondrial network, and the embedding of LDs within this network, is thought to be relevant for an efficient and homogenous distribution of fatty acids coming from these LDs across the mitochondrial population. Indeed, it has been shown in the case of cellular starvation, when cells rely heavily on oxidation of cellular stored lipid for energy production, that when mitochondrial fusion was inhibited, fatty acids were not homogenously distributed across mitochondria and fatty acid oxidation was reduced (Rambold et al., 2015). 


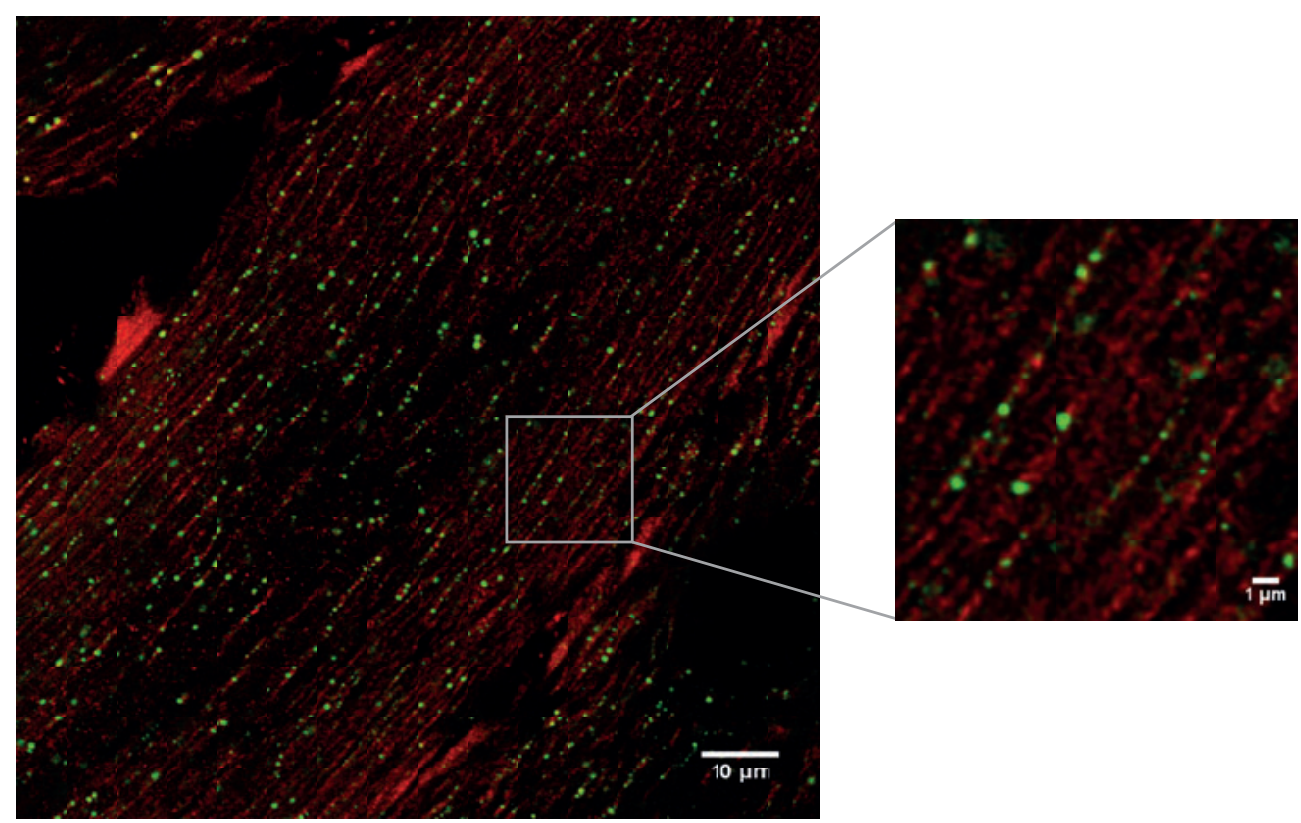

Figure 2. Lipid droplets (LDs) appear to be located within the mitochondrial network. The embedding of LDs within strings of mitochondria may facilitate efficient shuttling of fatty acids from the LDs toward the mitochondrial network in times of elevated energy demand. Immunofluorescence image of a longitudinal cryosection of a muscle cell in a human muscle biopsy of the vastus lateralis muscle. Mitochondria were stained using an antibody against a protein located in the outer mitochondrial membrane (translocase of the outer mitochondrial membrane-20, TOMM20). A secondary antibody labelled with Alexa Fluor 555 was used to visualize TOMM20. LDs have been stained green using Bodipy 493/503.

\section{Lipid composition and intermediates}

The negative relationship between IMCL and insulin sensitivity has been suggested to originate from an accumulation of toxic lipid intermediates. LDs may protect against lipid-induced insulin resistance via the sequestration of insulin-desensitizing lipid intermediates. In recent years, much attention has been given to the putative role of specific lipid classes, mainly DAG and ceramides, which impede insulin signalling in vitro and in animal studies (Schmitz-Peiffer et al., 1999; Montell et al., 2001). The relationship between these lipid classes and insulin resistance is not straightforward. Recent advances in lipidomics triggered examination of specific lipid intermediates with respect to fatty acid chain length, saturation and position on the glycerol backbone. 


\section{Diacylglycerols}

Our knowledge of the potential mechanisms by which DAGs may affect insulin resistance is primarily derived from in vitro and animal data. The prevailing idea is that DAG activates protein kinase C (PKC) isoforms, promoting serine phosphorylation of the insulin receptor substrate 1 (IRS-1), thereby blunting insulin-stimulated tyrosine phosphorylation and activation by phosphatidylinositol-3 kinase (PI3K), reducing localization of the glucose transporter GLUT4 to the cell membrane, and finally resulting in compromised glucose uptake (Erion \& Shulman, 2010).

Human intervention and cross-sectional studies investigating the possible association between DAG and insulin resistance have revealed conflicting results. Several studies have examined the role of DAG in acute lipid-induced insulin resistance. A pioneering study in humans, in which lipids were infused, revealed elevated DAG levels and a concomitant drop in insulin sensitivity (Itani et al., 2002). However, other studies failed to show elevated levels of DAG after lipid infusion while insulin sensitivity was clearly compromised (Vistisen et al., 2008). These observations dissociate DAG and acute lipid-induced insulin resistance.

Although several cross-sectional studies have shown higher total muscle DAG levels in obese and/or diabetic subjects and a negative correlation between total DAG levels and insulin sensitivity (Straczkowski et al., 2007; Moro et al., 2009; Bergman et al., 2012) other studies have failed to do so (Anastasiou et al., 2009; Perreault et al., 2010). The role of exercise training in modulating lipid composition has also shown conflicting results. Acute exercise blunted lipid infusion-mediated increases in DAG (Schenk \& Horowitz, 2007; Vistisen et al., 2008). However, in cross-sectional studies in endurance-trained subjects, DAG content was found to be similar (Bergman et al., 2010) or elevated (Amati et al., 2011) compared with that of sedentary subjects. Controversially, a moderate exercise training program in obese sedentary volunteers reduced total DAG levels (Dube et al., 2008). 


\section{Chapter 2}

Part of these inconsistencies may originate from differences in subspecies of DAG. Early biochemical studies highlighted the importance of both saturation and chain length of the fatty acid moieties in the bioactivity of DAG (Mori et al., 1982; Molleyres \& Rando, 1988). In insulin-resistant men, DAGs have a higher level of saturation compared with DAGs of insulin-sensitive controls. This was predominantly reflected by higher C16:0 and lower C18:1 DAG (van Hees et al., 2011). However, a significant increase in 18:1 DAG was found in obese versus non-obese subjects (Moro et al., 2009). Acute lipid-induced insulin resistance via lipid infusion led to an increase in DAG containing C16:0, C18:0, C18:1, C18:2 and C20:4; these changes correlated with the activation of PKC $\theta$ and insulin resistance. In another study, these DAG species were also associated with insulin resistance in obese and type 2 diabetes subjects (Szendroedi et al., 2014). In this study, both membrane-bound as cytosolic DAG was elevated (Szendroedi et al., 2014) and correlations with insulin resistance were most prominent for membrane fractions of DAG containing C18:1, C18:2 and C20:4. This observation matches another study reporting a negative correlation between membrane-bound DAG and insulin sensitivity (Bergman et al., 2012). Analysis of specific DAG subspecies in both compartments revealed elevated membrane DAG species C18:0/C20:4, di-C16:0 and di-C18:0 in patients with type 2 diabetes, with di-C18:0 showing a significant positive correlation with both insulin sensitivity and PKC $\varepsilon$ (Bergman et al., 2012). These studies highlight the importance of cellular localization of lipid species (see also the 'Location of lipids' section).

DAG species cannot only be distinguished on the basis of their fatty acid moieties, but also on the position of these moieties on the glycerol backbone, namely 1,2-DAG, 1,3-DAG and 2,3-DAG. The location and production of these isomers has been shown to be different. In general, three locations can be distinguished. DAG located at the plasma membrane consists of 1,2-DAG and is mainly generated from phospholipids. In addition, 1,2-DAG can be found at the ER and Golgi network and comes primarily from de novo lipogenesis. 2,3-DAG and 1,3-DAG are produced from TAG hydrolysis and, therefore, are present in cytosolic LDs (Eichmann \& Lass, 2015). Interestingly, early biochemical studies showed that only 1,2-DAG (not 1,3-DAG or 2,3-DAG) has 
the ability to activate PKC (Boni \& Rando, 1985; Hannun et al., 1986) and, hence, impede insulin signalling and contribute to insulin resistance. However, no such data exist specifically for intact cells systems, let alone for skeletal muscle. To date, no human studies have reported on the specific positions of the fatty acid moieties on the glycerol backbone. Some indication of the importance of fatty acid position comes from a study using hormone-sensitive lipase (HSL) knock-out mice. After a treadmill exercise, knock-out mice showed increased insulin-stimulated glucose uptake along with higher (rather than lower) total DAG levels compared with wild-type mice. DAG subtype analysis revealed that 1,3-DAG increased after exercise, whereas 1,2DAG decreased after exercise (Serup et al., 2016).

\section{Ceramides}

Ceramides have a central role in sphingolipid metabolism given that they are a precursor for complex sphingolipids. They can be produced de novo from serine and saturated fatty acids, mainly palmitate (C16), and from sphingomyelin hydrolysis, or they are reformed via the salvage pathway from complex sphingolipids (Bellini et al., 2015). As de novo synthesis of ceramides requires fatty acids such as palmitate, the rate of ceramide synthesis is dependent on the availability of these fatty acids. In this respect it has been shown that diet high in saturated fat can influence intramyocellular ceramide accumulation (Blachnio-Zabielska et al., 2010). As with DAGs, the mechanism by which ceramides are thought to induce insulin resistance is mainly derived from in vitro and animal studies. Ceramides may inhibit insulin-stimulated glucose uptake via the inhibition of Akt/protein kinase B (PKB) activation. Two routes have been proposed, namely the activation of phosphatase 2A by ceramides, leading to dephosphorylation of Akt/PKB (Stratford et al., 2004), and by activation of the atypical PKC isoform PKC $\zeta$, which binds, phosphorylates and sequesters Akt/PKB in caveolin-enriched microdomains (Powell et al., 2003; Fox et al., 2007).

Several studies in humans support a relationship between ceramide accumulation and insulin resistance. An inverse relationship between total muscle ceramide content and insulin sensitivity was found in lean, insulin-sensitive subjects (Straczkowski 


\section{Chapter 2}

et al., 2004). In addition, ceramide levels increased in the muscle of obese subjects (Adams et al., 2004; Straczkowski et al., 2007; Coen et al., 2010) and the offspring of type 2 diabetes patients (Straczkowski et al., 2007) compared with lean subjects. Two studies have shown that participation in a moderate exercise training program can reduce the total ceramide levels of obese sedentary individuals and improve insulin sensitivity (Bruce et al., 2006; Dube et al., 2008).

However, other studies have not reported a link between ceramides and insulin resistance. Upon examining muscle ceramide content in patients with a wide range of insulin sensitivity, only a weak borderline significant correlation between ceramide levels and insulin sensitivity was observed (Skovbro et al., 2008). Acute induction of insulin resistance by lipid infusion was observed without changes in ceramide levels ceramides (Hoeks et al., 2012). In addition, several intervention studies, including weight loss and/or exercise training, failed to show any changes in muscle ceramides (Dube et al., 2011; Helge et al., 2011; Johnson et al., 2016; Sogaard et al., 2016).

Rather than total ceramide content, specific ceramide species may be responsible for the effect on insulin sensitivity. Thus, although the total muscle ceramide levels of type 2 diabetes patients, obese individuals and endurance-trained athletes are the same, C18:0 ceramide has been reported to be higher in the muscle of type 2 diabetes patients relative to that of obese individuals and endurance-trained athletes. Moreover, other C18:0 sphingolipids (dihydroceramide and glucosylceramide) have been associated with insulin resistance (Bergman et al., 2016). The association between C18:0 ceramides and insulin resistance was extended by a study showing associations between insulin resistance and ceramide di18:1/18:0 and C18:0 sphingolipids dihexosylceramide and trihexosylceramide (Tonks et al., 2016). Furthermore, ceramides C14:0, C16:0 and C18:0 were increased in obese insulin-resistant women compared with those of obese insulin-sensitive women (Coen et al., 2010). In addition to these long-chain ceramides, very long-chain ceramides (20:0, 20:4 (de la Maza et al., 2015), c20:1 and C22:1 (Thrush et al., 2009)) have also been associated with obesity and glucose intolerance. As well as the ceramides subtype, the subcellular location of ceramides might also be of importance. For example, ceramides have 
been implicated to be present in mitochondrial membranes and contribute to mitochondrial dysfunction (Di Paola et al., 2000; Yu et al., 2007) which indirectly links ceramides to insulin resistance. However, there are no data on the subcellular location of ceramides in human skeletal muscle.

In general, the evidence for a direct role of diacylglycerols and ceramides in human muscle insulin resistance is inconsistent at best. With recent advances in lipidomics, it has become evident that specific lipid intermediates may be important, which may explain inconsistencies between earlier studies looking at total DAG or ceramides. In addition, most data, particularly in human studies, are correlative and causality is difficult to prove. Moreover, the metabolism of lipid intermediates is highly intertwined, which complicates the identification of 'malign' insulin-desensitizing lipid moieties. Besides this, the role of specific lipid intermediates might be gender-, situation-, intervention- or even (epi)genetic-specific (Kitessa \& Abeywardena, 2016).

\section{Location of lipids}

Another complicating factor for the interpretation of changes in insulin-desensitizing bioactive lipids is the observation that the subcellular location of these lipid moieties appears to be of relevance. Skeletal muscle contains glycolytic non-oxidative muscle fibres (type II fibres) and oxidative muscle fibres that are well equipped for lipid oxidation (type I fibres). The fibre type specificity of LD distribution (with more LDs being present in type I fibres) can be readily observed in histology (Figure 3A-C). Indeed, skeletal muscle fibre typology is related to lipid content, oxidative capacity (He et al., 2001) and insulin sensitivity (Albers et al., 2015). Although several studies in rodents have indicated differences in the lipid storage pattern between oxidative and glycolytic muscle (Lally et al., 2012; Holloway et al., 2014), human studies looking at fibre type-specific IMCL storage and its relation to insulin sensitivity are limited. Two studies showed larger and fewer LDs in type I fibres of type 2 diabetes patients than in lean sedentary individuals and endurance-trained athletes (He et al., 2004; van Loon et al., 2004). In addition, IMCL content was higher in type I fibres in insulinresistant subjects than in insulin-sensitive obese subjects. Moreover, IMCL content 
correlated negatively with insulin sensitivity only in type I fibres (Coen et al., 2010). Furthermore, the subcellular location of LDs (in the intermyofibrillar (IMF) space and in the vicinity of mitochondria or in the subsarcolemmal (SS) space, Figure 3D) may be a determinant of insulin sensitivity.
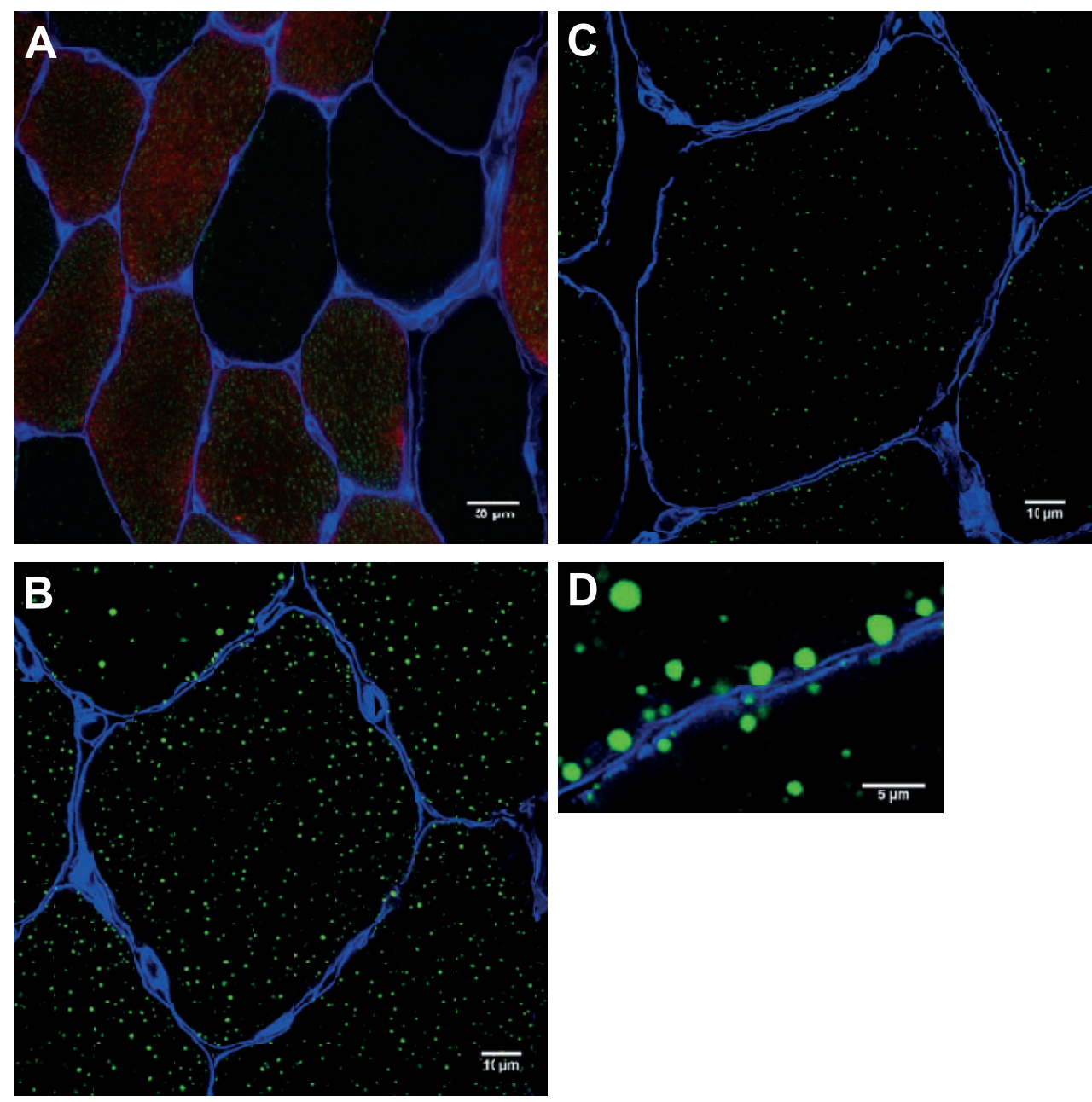

Figure 3. Distribution of lipid droplets (LDs) in skeletal muscle. A) Muscle lipid storage differs between muscle fibre types. Widefield microscopy image of human skeletal muscle fibres (vastus lateralis muscle) in cross-sectional view. The basement membrane protein laminin is shown in blue (secondary antibody labelled with Alexa Fluor 405), LDs are stained green (Bodipy 493/503), and type I fibres are shown in red (primary antibody against myosin heavy chain type 1; secondary antibody labelled with Alexa Fluor 555). $\boldsymbol{B}$ and $\mathbf{C})$ Confocal images of human muscle fibres in cross-sectional view. In human skeletal muscle, $L D$ s are more abundant in type I muscle fibres (B) than in type II fibres (C). The staining procedures were similar to the staining described for $3 A$. D) Subcellularly, LDs are dispersed alongside the contractile elements but are also in the vicinity of the muscle cell membrane.taining procedures were similar to the staining described for $3 A$. 
The importance of subcellular location has already been illustrated above by the possible differences in the subcellular location of DAGs. Other studies also hint at the importance of subcellular LD location with respect to insulin sensitivity. Type 2 diabetes patients have been shown to have increased IMCL content, compared with obese and endurance-trained subjects, in the SS region. The presence of SS LDs negatively correlated with insulin resistance in type 2 diabetes patients (Nielsen et al., 2010). Furthermore, a training intervention decreased LD content in the SS area in lean, obese (Samjoo et al., 2013; Li et al., 2014) and type 2 diabetes subjects (Nielsen et al., 2010). However, exactly how LD location affects insulin sensitivity remains elusive.

The location of LDs is also a determinant of the interaction of LDs with other cellular organelles (such as the ER). Thus, LD location also affects subcellular lipid handling and metabolism. For organelle interactions to occur, organelle motility is essential to bring organelles into close proximity and direct membrane connections are warranted. ER-LD contacts may facilitate the transfer of bioactive lipids and of proteins with metabolic or signalling functions (Barbosa et al., 2015). As an example, several TAG synthetic enzymes such as diacylglycerol-O-acyltransferase 2 (DGAT2) are bound to the ER membrane and move to the LD to promote TAG synthesis (Kuerschner et al., 2008; Barbosa et al., 2015). Although data on the motility of LDs in human skeletal muscle are scarce, LD motility is anticipated to facilitate fluctuations in energy supply and demand, e.g. during exercise (Badin et al., 2013).

Classically, lipid composition analysis entails tissue lipid extraction, followed by conventional mass spectrometry. Unavoidably, this procedure results in the loss of all spatial information. However, several techniques are emerging that enable both chemical and spatial information to be analysed. Advances in both vibrational microscopy, e.g. coherent anti-stokes Raman scattering (CARS), and imaging mass spectrometry, e.g. secondary ion mass spectrometry (SIMS), could enable the role of specific lipid species in their subcellular localization to be studied in the near future (Daemen et al., 2016). 


\section{Dietary effects on lipid droplets and intramyocellular lipid content}

A sustained positive energy balance (e.g. via the excess caloric intake) results in the development of overweight and/or obesity. Obesity development and the concomitant expansion of white adipose tissue mass promotes the storage of fat in a variety of tissues, including skeletal muscle. Thus, elevated IMCL content is commonly observed in the obese state. Somewhat counterintuitively, prolonged fasting or alternate day fasting also seems to augment IMCL content. The following section aims to outline how dietary interventions can affect IMCL content.

\section{High fat diet and lipid infusion}

A hypercaloric high-fat diet (HFD) can promote IMCL content in resting muscle within days (Bachmann et al., 2001; Zderic et al., 2004; Schrauwen-Hinderling et al., 2005; Zehnder et al., 2006; Larson-Meyer et al., 2008; Sakurai et al., 2011; Van Proeyen et al., 2011). If provided after an exercise session, a HFD rapidly restores the exercisemediated drop in IMCL (Decombaz et al., 2001; Larson-Meyer et al., 2002; van Loon et al., 2003b). Thus, the consumption of a HFD promotes myocellular fat deposition, even during periods that are too short to significantly affect body mass.

Nevertheless, it is interesting to examine models in which IMCL content was acutely (within hours) elevated by the infusion of lipid emulsions. These models compared the effects of intravenous lipid emulsion infusions on IMCL content with those of saline or glycerol infusions. Lipid emulsions were co-infused with or without heparin to stimulate endothelial lipoprotein lipase (LPL) activity. Typically, this results in elevated plasma FFA levels up to the millimolar range (compared with 200-300 $\mu \mathrm{mol}$ under control conditions). These studies have robustly and consistently shown that infusion of lipid emulsions results in a significant increase in IMCL content (Bachmann et al., 2001; Brechtel et al., 2001a; Brehm et al., 2010; Hoeks et al., 2012; Lee et al., 2013) in type I type II muscle fibres of healthy male individuals (Hoeks et al., 2012) and highly trained athletes (Phielix et al., 2012). In these lipid emulsion 
infusion studies, the increase in IMCL content was paralleled by a decrease in insulinstimulated glucose uptake in muscle. Interestingly, in trained athletes, lipid-induced insulin resistance was ameliorated (Phielix et al., 2012) even though IMCL content was significantly increased. Studies involving a high-protein/low-carbohydrate diet did not alter plasma FFA levels and failed to affect IMCL content or whole-body insulin sensitivity (Green et al., 2010).

Thus, elevating circulatory FFA levels in situations of a positive energy balance seems to promote TAG storage in skeletal muscle and compromise insulin sensitivity in men with normal to low oxidative capacity as well as in athletes. Cycling exercise for 3 hours in the fasted state reduces IMCL content in the active muscles and is paralleled by increased plasma FFA levels and, interestingly, by increased IMCL content in the inactive muscle (Schrauwen-Hinderling et al., 2003b). This indicates that IMCL not only represents a very mobile lipid pool, but also supports the notion that elevated plasma FFA levels may drive IMCL content.

\section{Calorie restriction and diet-induced weight loss}

Calorie restriction is an effective non-pharmaceutical intervention to improve life span and age-related diseases in various organisms. In rodents, a 30-50\% reduction in calorie intake can lead to improved health (Guarente, 2006; Carmona \& Michan, 2016). The influence of calorie reduction or weight loss interventions on IMCL has been studied in relation to insulin sensitivity in several populations.

Overweight and obese people subjected to a diet-induced weight loss (DIWL) intervention (16 weeks) showed a drop in IMCL and a parallel improvement in insulin sensitivity (Toledo et al., 2008). In another study with a similar design, overweight individuals not only showed a reduction in total IMCL but also a parallel drop in DAG content and in specific ceramides (although total ceramide levels remained unchanged (Dube et al., 2011)). These data, along with a study showing similar effects upon DIWL (Lara-Castro et al., 2008) indicate that DIWL potentially reduces IMCL content and affects its composition. These changes appear to occur in a muscle fibre 


\section{Chapter 2}

type-independent fashion (Gray et al., 2003) and occur in parallel to a substantial improvement in insulin sensitivity (Larson-Meyer et al., 2006).

Caloric restriction not only reduces IMCL content in healthy individuals. Insulinresistant offspring of patients with type 2 diabetes on a hypocaloric diet for 9 weeks also showed a reduced total IMCL content (owing to reductions in LD size, not number) and improved insulin sensitivity (Petersen et al., 2012). In patients with type 2 diabetes, a 6-day very low-calorie diet reduced IMCL content by 53\%, whereas insulin-stimulated glucose uptake increased by $9 \%$ (Lara-Castro et al., 2008). In obese patients with type 2 diabetes, a very low-calorie diet with targeted weight loss reduced IMCL and improved insulin sensitivity (Jazet et al., 2008). In morbidly obese patients eligible for gastric bypass surgery, the intervention reduced their BMI by $43 \%$ with a concomitant drop in IMCL of 30\% and improvements in glucose homeostasis (Gray et al., 2003). In most of these studies, body mass also decreased significantly. Thus, it is premature to conclude that the improvement in glucose homeostasis observed after caloric restriction entirely originates from a reduction in IMCL.

\section{Fasting}

In models of caloric restriction (e.g. alternate day fasting, very low calorie diet or gastric bypass), food is still consumed and, hence, there are transient cycles of carbohydrate and insulin peaks. During prolonged fasting, these oscillations are absent and the body relies on endogenous energy stores. Thus, hydrolysis of TAG in adipose tissue results in elevated plasma FFA levels. This elevation of plasma FFA levels is probably responsible for the observation that (in contrast to caloric restriction) prolonged fasting (>48 hours) augments IMCL levels in healthy individuals (Stannard et al., 2002; Wietek et al., 2004; Green et al., 2010; Hoeks et al., 2010; Browning et al., 2012; Gemmink et al., 2016). Shorter episodes of fasting ( $<12$ hours), requiring only a modest activation of adipose tissue lipolysis, did not result in increased IMCL levels (Machann et al., 2011). This apparent discrepancy is explained by lower plasma FFA levels in the early phase of fasting (Stannard et al., 2002; Wietek et al., 2004; Green et al., 2010; Hoeks et al., 2010; Browning et al., 2012). Recently, we showed that following 
prolonged (62 hours) fasting, when plasma FFA levels were substantially elevated, LDs coated with PLIN5 significantly increased in size compared with LDs that were not coated with PLIN5 (Gemmink et al., 2016). We also observed that participants that showed the greatest increase in LD size were least insulin resistant upon fasting (Gemmink et al., 2016). Thus, prolonged fasting results in elevated plasma FFA levels and augments IMCL content; however, if IMCL is stored in PLIN5-coated LDs, the increase in IMCL does not compromise insulin sensitivity.

\section{Other dietary interventions}

Most commonly, the macronutrient composition of the diet is changed to modulate IMCL levels (changes in total caloric intake, or changes in the fat component of the diet). Alternatively, potentially bioactive food compounds can be selectively administered. We previously observed that obese normoglycemic patients administered with polyphenol resveratrol for 30 days showed a significant increase in IMCL content (Timmers et al., 2011). The increase in IMCL was most pronounced in type I muscle fibres, and a parallel increase in the protein content of the LD coat protein PLIN5 and a decrease in hepatic fat content were also observed (Timmers et al., 2011). Similar observations were made in a follow-up study in patients with type 2 diabetes (Timmers et al., 2016). How resveratrol exerts these effects is unknown. Intriguingly, the low hepatic fat content and elevated IMCL levels in type I muscle fibres, along with the induction of PLIN5, mimic an exercise signature for LD remodelling (Koves et al., 2013) suggesting that resveratrol affects IMCL levels in a manner that is commonly associated with health benefits.

Thus, there is a growing body of literature that recognizes the importance of nutrition in LD synthesis and the modulation of the total IMCL pool in muscle. However, most of the research on the effects of diet and IMCL have focused on static measures of IMCL and have not taken into account factors involved in the dynamic nature of LDs. In a study involving a combined exercise training and dietary intervention, LD size was reduced (He et al., 2004). This drop in LD size correlated with an improvement in insulin sensitivity (He et al., 2004) suggesting that the size of LDs somehow affects 


\section{Chapter 2}

insulin sensitivity. How dietary interventions affect LD dynamics and their coating proteins in relation to insulin sensitivity remains elusive.

\section{Effects of acute exercise on IMCL}

After acute exercise at moderate intensity, most studies report not only a decrease in IMCL in healthy and sedentary subjects (Decombaz et al., 2001; SchrauwenHinderling et al., 2003a; White et al., 2003; Egger et al., 2013; Bucher et al., 2014) but also in endurance-trained athletes (Krssak et al., 2000; Rico-Sanz et al., 2000; Brechtel et al., 2001b; Decombaz et al., 2001; Johnson et al., 2003; van Loon et al., 2003a; van Loon et al., 2003b; Zehnder et al., 2005; De Bock et al., 2007; Vermathen et al., 2012). Importantly, the utilization of IMCL during exercise depends upon the duration and intensity of exercise and the training status of the participant. All these factors affect the degree of stimulation of lipolysis of IMCL and fat oxidative capacity. During prolonged, moderate-intensity endurance exercise, fat oxidation is maximal (Kiens et al., 2011). However, even at maximal rates of fat oxidation, there is still a substantial contribution of plasma-derived fatty acids to total fat oxidation (van Loon et al., 2003a). The relative contribution of IMCL and plasma sources to lipid oxidation during exercise remains a subject of investigation and may vary with nutritional and training status and exercise intensity.

With respect to dietary status, subjects showed a reduction in IMCL after a 2 hour cycling exercise at $75 \% \mathrm{VO}_{2}$ max only under fasting conditions and not when fed carbohydrates. Moreover, this decrease was only seen in type I fibres (De Bock et al., 2005). Another study examined the effect on subjects of 48 hours of high- or low-dietary carbohydrate before exercise and found higher net IMCL usage in the low-carbohydrate condition, mainly owing to higher IMCL levels before exercise started (Johnson et al., 2003). Furthermore, it was shown that a HFD increased net IMCL degradation during exercise, an effect that was not altered by an exercise training program (Van Proeyen et al., 2011), again possibly owing to increased basal IMCL values. Indeed, several studies indicate that it may be basal IMCL values that are the determinant of IMCL usage during exercise (Steffensen et al., 2002; Zehnder 
et al., 2006; Vermathen et al., 2012), so higher IMCL levels at the onset of exercise may result in higher IMCL utilization. For example, athletes given 1.5 days of lipid supplementation before an acute cycling exercise showed increased pre-exercise IMCL levels; the IMCL depletion during exercise was strongly correlated with these pre-exercise IMCL levels (Zehnder et al., 2006). Furthermore, training status can influence the amount of IMCL used during exercise: for example, trained subjects showed higher levels of fat oxidation during $30 \mathrm{~min}$ of exercise at $75-80 \%$ of $\mathrm{VO}_{2}$ max than untrained individuals, which could not be completely explained by higher usage of plasma FFA (Coggan et al., 2000). This additional lipid substrate must therefore come from intramuscular fat stores, presumably IMCL.

Although the effects of an acute exercise bout on total IMCL content have been extensively studied, the effect of exercise on LD dynamics and their coating proteins during acute exercise in humans has not yet been studied in great detail. One study showed that $60 \mathrm{~min}$ of cycling at $60 \%$ of $\mathrm{VO}_{2}$ max did not alter the interaction of PLIN5, ATGL and its co-activators with LDs (Mason et al., 2014). Another study showed that LDs coated with PLIN5 were depleted during exercise, whereas LDs without PLIN5 were not (Shepherd et al., 2012). This selective use was maintained by a 6-week sprint interval training or endurance training intervention (Shepherd et al., 2012). Similarly, PLIN2-coated LDs were used during acute exercise in contrast to LDs devoid of PLIN2 (Shepherd et al., 2012, 2013). In contrast to PLIN5, the preference for utilizing PLIN2-positive LDs over LDs devoid of PLIN2 faded upon 6-week sprint interval training, as well as after the endurance training program (Shepherd et al., 2013).

Thus, during acute physical exercise, IMCL is used to fuel the increased energy demand of exercise and, hence, IMCL levels drop. The level of IMCL utilization during acute exercise depends on nutritional status, training status, absolute and relative exercise intensity and the IMCL levels at the onset of exercise. More detailed analyses of LDs during acute exercise indicate that coating LDs with PLIN2 or PLIN5 also affects the utilization of IMCL during exercise. 


\section{Long-term exercise training effects}

In most populations, chronic exercise training augments IMCL storage (Phillips et al., 1996; Schrauwen-Hinderling et al., 2003a; Pruchnic et al., 2004; Tarnopolsky et al., 2007; Dube et al., 2008; Shepherd et al., 2013). This appears to be in line with the 'athlete's paradox' and the notion that IMCL fuels exercise of moderate intensity. However, in type 2 diabetes patients and impaired glucose-tolerant subjects, the effects of exercise training on IMCL content are less consistent. Although some studies report augmented IMCL storage upon exercise training (Meex et al., 2010; Peters et al., 2012), others have reported no change (Jonker et al., 2013) or a reduction (Bruce et al., 2004; Solomon et al., 2008). Although these apparent differences may originate from different training protocols, they might also be a reflection of the limited exercise capacity of patients with type 2 diabetes (Stephens \& Sparks, 2015). The lower exercise capacity of type 2 diabetes patients blunts the reliance on IMCL as a substrate (Ghanassia et al., 2006) and, hence, may not suffice as a trigger to augment IMCL content. However, even in the absence of reductions in IMCL, training improves insulin sensitivity (Bruce et al., 2004; Dube et al., 2008; Solomon et al., 2008; Nielsen et al., 2010).

The insulin-sensitizing effect of exercise training does not require changes in total IMCL content. The emerging view is that alterations in LD morphology, coating LDs with proteins of the perilipin family, the subcellular localization of the LD and the LD-mitochondrial interaction are more directly related to the insulin-sensitizing effect of exercise than to total IMCL content. In lean men and women, 7 weeks of endurance exercise training increased IMCL content owing to an increase in the number of LDs rather than an increase in LD size (Tarnopolsky et al., 2007). This increase in LD number rather than LD size was also observed in another study (Shepherd et al., 2013). Furthermore, several studies have observed an improvement in the contact between LDs and mitochondria (Tarnopolsky et al., 2007; Devries et al., 2013). With regard to the coating of LDs, the expression of PLIN2 and PLIN5 increases upon exercise training (Gjelstad et al., 2012; Peters et al., 2012; Shaw et al., 2012; Shepherd et al., 2013); one study also reported an increase in the expression of PLIN3 (Gjelstad 
et al., 2012). However, the fraction of PLIN2 and PLIN5 co-localizing with IMCL remained unchanged (Shepherd et al., 2013).

Exercise training also appears to lead to a remodelling of cellular lipid distribution. In both obese non-diabetic and lean women, the redistribution of lipid from SS to IMF was observed after training, alongside an increased LD-mitochondrial interaction, together most likely favouring lipid oxidation during exercise (Devries et al., 2013). In overweight dysglycemic and normal weight control subjects, SS LDs, as opposed to IMF LDs, decreased both in size and number (Li et al., 2014). Moreover, type 2 diabetes patients showed higher baseline SS lipid levels compared with obese controls and endurance-trained athletes. The training intervention reduced the SS lipid levels by half in these diabetic patients, reaching the levels of non-diabetic controls (Nielsen et al., 2010).

Although the effect of acute exercise and exercise training on total IMCL levels has been extensively studied, further studies are needed to elucidate the role of proteins involved in LD dynamics on IMCL turnover during exercise, and to explore whether these processes are affected in the insulin-resistant state.

\section{Conclusions and future perspectives}

In contrast to what has been the dogma for years, IMCL represents a highly dynamic pool of lipids that can readily adjust to alterations in energy supply and demand. A sustained positive energy balance promotes IMCL, whereas intermittent periods of caloric restriction or short-term fasting reduces the IMCL pool size. In general, high IMCL levels are associated with impeded insulin sensitivity; however, this is not always the case. If, for example, IMCL is elevated because of exercise training, the insulin desensitizing effect is not apparent. In the trained state, the IMCL pool undergoes continuous cycles of depletion and repletion and seems to have more intimate interaction with organelles such as mitochondria and the sarcoplasmic reticulum. These adaptive responses enable rapid esterification of fatty acids entering the myocyte and rapid oxidation of fatty acids released from the LD upon lipase 


\section{Chapter 2}

activity. To maintain the dynamic nature of LDs, an orchestrated interaction of lipases such as HSL and ATGL, their coactivators, and the myocellular members of the PLIN family (PLIN2, PLIN3 and PLIN5) is essential. We are just beginning to understand how these proteins respond to dietary and exercise interventions and how they affect LD dynamics. However, to date, the results seem to indicate that by modulating these proteins either by diet, nutritional compounds or exercise, the LD phenotype can be affected such that expansion of IMCL can occur without negative effects on cellular function and/or insulin sensitivity. Clearly, more work needs to be done to determine how and why alterations in proteins involved in LD dynamics affect LD and cellular function and under which conditions these changes can be beneficial to human health. 


\section{References}

Adams JM, 2nd, Pratipanawatr T, Berria R, Wang E, DeFronzo RA, Sullards MC \& Mandarino LJ. (2004). Ceramide content is increased in skeletal muscle from obese insulinresistant humans. Diabetes 53, 25-31.

Albers PH, Pedersen AJ, Birk JB, Kristensen DE, Vind BF, Baba O, Nohr J, Hojlund K \& Wojtaszewski JF. (2015). Human muscle fiber type-specific insulin signaling: impact of obesity and type 2 diabetes. Diabetes 64, 485-497.

Amati F, Dube JJ, Alvarez-Carnero E, Edreira MM, Chomentowski P, Coen PM, Switzer GE, Bickel PE, Stefanovic-Racic M, Toledo FG \& Goodpaster BH. (2011). Skeletal muscle triglycerides, diacylglycerols, and ceramides in insulin resistance: another paradox in endurance-trained athletes? Diabetes 60, 2588-2597.

Anastasiou CA, Kavouras SA, Lentzas Y, Gova A, Sidossis LS \& Melidonis A. (2009). Diabetes mellitus is associated with increased intramyocellular triglyceride, but not diglyceride, content in obese humans. Metabolism: clinical and experimental 58, 1636-1642.

Anderwald C, Bernroider E, Krssak M, Stingl H, Brehm A, Bischof MG, Nowotny P, Roden M \& Waldhausl W. (2002). Effects of insulin treatment in type 2 diabetic patients on intracellular lipid content in liver and skeletal muscle. Diabetes 51, 3025-3032.

Bachmann OP, Dahl DB, Brechtel K, Machann J, Haap M, Maier T, Loviscach M, Stumvoll M, Claussen CD, Schick F, Haring HU \& Jacob S. (2001). Effects of intravenous and dietary lipid challenge on intramyocellular lipid content and the relation with insulin sensitivity in humans. Diabetes 50, 2579-2584.

Badin PM, Langin D \& Moro C. (2013). Dynamics of skeletal muscle lipid pools. Trends in endocrinology and metabolism: TEM 24, 607-615.

Barbosa AD, Savage DB \& Siniossoglou S. (2015). Lipid droplet-organelle interactions: emerging roles in lipid metabolism. Current opinion in cell biology 35, 91-97.

Bellini L, Campana M, Mahfouz R, Carlier A, Veret J, Magnan C, Hajduch E \& Le Stunff H. (2015). Targeting sphingolipid metabolism in the treatment of obesity/type 2 diabetes. Expert opinion on therapeutic targets 19, 1037-1050.

Bergman BC, Brozinick JT, Strauss A, Bacon S, Kerege A, Bui HH, Sanders P, Siddall P, Wei T, Thomas MK, Kuo MS \& Perreault L. (2016). Muscle sphingolipids during rest and exercise: a C18:0 signature for insulin resistance in humans. Diabetologia 59, 785-798.

Bergman BC, Hunerdosse DM, Kerege A, Playdon MC \& Perreault L. (2012). Localisation and composition of skeletal muscle diacylglycerol predicts insulin resistance in humans. Diabetologia 55, 1140-1150.

Bergman BC, Perreault L, Hunerdosse DM, Koehler MC, Samek AM \& Eckel RH. (2010). Increased intramuscular lipid synthesis and low saturation relate to insulin sensitivity in endurance-trained athletes. Journal of applied physiology 108, 1134-1141.

Blaak EE. (2004). Basic disturbances in skeletal muscle fatty acid metabolism in obesity and type 2 diabetes mellitus. Proc Nutr Soc 63, 323-330.

Blaak EE \& Wagenmakers AJ. (2002). The fate of [U-(13)C]palmitate extracted by skeletal muscle in subjects with type 2 diabetes and control subjects. Diabetes 51, 784-789.

Blachnio-Zabielska A, Baranowski M, Zabielski P \& Gorski J. (2010). Effect of high fat diet enriched with unsaturated and diet rich in saturated fatty acids on sphingolipid metabolism in rat skeletal muscle. Journal of cellular physiology 225, 786-791. 
Boni LT \& Rando RR. (1985). The nature of protein kinase C activation by physically defined phospholipid vesicles and diacylglycerols. The Journal of biological chemistry 260, 10819-10825.

Bosma M, Hesselink MK, Sparks LM, Timmers S, Ferraz MJ, Mattijssen F, van Beurden D, Schaart G, de Baets MH, Verheyen FK, Kersten S \& Schrauwen P. (2012a). Perilipin 2 improves insulin sensitivity in skeletal muscle despite elevated intramuscular lipid levels. Diabetes 61, 2679-2690.

Bosma M, Minnaard R, Sparks LM, Schaart G, Losen M, de Baets MH, Duimel H, Kersten S, Bickel PE, Schrauwen P \& Hesselink MK. (2012b). The lipid droplet coat protein perilipin 5 also localizes to muscle mitochondria. Histochemistry and cell biology 137, 205-216.

Brasaemle DL, Barber T, Wolins NE, Serrero G, Blanchette-Mackie EJ \& Londos C. (1997). Adipose differentiation-related protein is an ubiquitously expressed lipid storage droplet-associated protein. Journal of lipid research 38, 2249-2263.

Brechtel K, Dahl DB, Machann J, Bachmann OP, Wenzel I, Maier T, Claussen CD, Haring HU, Jacob S \& Schick F. (2001a). Fast elevation of the intramyocellular lipid content in the presence of circulating free fatty acids and hyperinsulinemia: a dynamic ${ }^{1} \mathrm{H}-\mathrm{MRS}$ study. Magn Reson Med 45, 179-183.

Brechtel K, Niess AM, Machann J, Rett K, Schick F, Claussen CD, Dickhuth HH, Haering HU \& Jacob S. (2001b). Utilisation of intramyocellular lipids (IMCLs) during exercise as assessed by proton magnetic resonance spectroscopy ( $\left.{ }^{1} \mathrm{H}-\mathrm{MRS}\right)$. Horm Metab Res 33, 63-66.

Brehm A, Krssak M, Schmid AI, Nowotny P, Waldhausl W \& Roden M. (2010). Acute elevation of plasma lipids does not affect ATP synthesis in human skeletal muscle. Am J Physiol-Endoc M 299, E33-E38.

Browning JD, Baxter J, Satapati S \& Burgess SC. (2012). The effect of short-term fasting on liver and skeletal muscle lipid, glucose, and energy metabolism in healthy women and men. Journal of lipid research 53, 577-586.

Bruce CR, Kriketos AD, Cooney GJ \& Hawley JA. (2004). Disassociation of muscle triglyceride content and insulin sensitivity after exercise training in patients with Type 2 diabetes. Diabetologia 47, 23-30.

Bruce CR, Thrush AB, Mertz VA, Bezaire V, Chabowski A, Heigenhauser GJ \& Dyck DJ. (2006). Endurance training in obese humans improves glucose tolerance and mitochondrial fatty acid oxidation and alters muscle lipid content. American journal of physiology Endocrinology and metabolism 291, E99-E107.

Bucher J, Krusi M, Zueger T, Ith M, Stettler C, Diem P, Boesch C, Kreis R \& Christ E. (2014). The effect of a single $2 \mathrm{~h}$ bout of aerobic exercise on ectopic lipids in skeletal muscle, liver and the myocardium. Diabetologia 57, 1001-1005.

Carmona JJ \& Michan S. (2016). Biology of Healthy Aging and Longevity. Rev Invest Clin 68, 7-16.

Coen PM, Dube JJ, Amati F, Stefanovic-Racic M, Ferrell RE, Toledo FG \& Goodpaster BH. (2010). Insulin resistance is associated with higher intramyocellular triglycerides in type I but not type II myocytes concomitant with higher ceramide content. Diabetes $59,80-88$.

Coggan AR, Raguso CA, Gastaldelli A, Sidossis LS \& Yeckel CW. (2000). Fat metabolism during high-intensity exercise in endurance-trained and untrained men. Metabolism: 
clinical and experimental 49, 122-128.

Daemen S, van Zandvoort MA, Parekh SH \& Hesselink MK. (2016). Microscopy tools for the investigation of intracellular lipid storage and dynamics. Molecular metabolism 5, 153-163.

Dalen KT, Dahl T, Holter E, Arntsen B, Londos C, Sztalryd C \& Nebb HI. (2007). LSDP5 is a PAT protein specifically expressed in fatty acid oxidizing tissues. Biochim Biophys Acta 1771, 210-227.

De Bock K, Dresselaers T, Kiens B, Richter EA, Van Hecke P \& Hespel P. (2007). Evaluation of intramyocellular lipid breakdown during exercise by biochemical assay, NMR spectroscopy, and Oil Red O staining. American journal of physiology Endocrinology and metabolism 293, E428-434.

De Bock K, Richter EA, Russell AP, Eijnde BO, Derave W, Ramaekers M, Koninckx E, Leger B, Verhaeghe J \& Hespel P. (2005). Exercise in the fasted state facilitates fibre typespecific intramyocellular lipid breakdown and stimulates glycogen resynthesis in humans. J Physiol 564, 649-660.

de la Maza MP, Rodriguez JM, Hirsch S, Leiva L, Barrera G \& Bunout D. (2015). Skeletal muscle ceramide species in men with abdominal obesity. The journal of nutrition, health \& aging 19, 389-396.

Decombaz J, Schmitt B, Ith M, Decarli B, Diem P, Kreis R, Hoppeler H \& Boesch C. (2001). Postexercise fat intake repletes intramyocellular lipids but no faster in trained than in sedentary subjects. Am J Physiol Regul Integr Comp Physiol 281, R760-769.

Devries MC, Samjoo IA, Hamadeh MJ, McCready C, Raha S, Watt MJ, Steinberg GR \& Tarnopolsky MA. (2013). Endurance training modulates intramyocellular lipid compartmentalization and morphology in skeletal muscle of lean and obese women. The Journal of clinical endocrinology and metabolism 98, 4852-4862.

Di Paola M, Cocco T \& Lorusso M. (2000). Ceramide interaction with the respiratory chain of heart mitochondria. Biochemistry 39, 6660-6668.

Dube JJ, Amati F, Stefanovic-Racic M, Toledo FG, Sauers SE \& Goodpaster BH. (2008). Exerciseinduced alterations in intramyocellular lipids and insulin resistance: the athlete's paradox revisited. American journal of physiology Endocrinology and metabolism 294, E882-888.

Dube JJ, Amati F, Toledo FG, Stefanovic-Racic M, Rossi A, Coen P \& Goodpaster BH. (2011). Effects of weight loss and exercise on insulin resistance, and intramyocellular triacylglycerol, diacylglycerol and ceramide. Diabetologia 54, 1147-1156.

Egger A, Kreis R, Allemann S, Stettler C, Diem P, Buehler T, Boesch C \& Christ ER. (2013). The effect of aerobic exercise on intrahepatocellular and intramyocellular lipids in healthy subjects. PloS one 8, e70865.

Eichmann TO \& Lass A. (2015). DAG tales: the multiple faces of diacylglycerol--stereochemistry, metabolism, and signaling. Cellular and molecular life sciences : CMLS 72, 3931-3952.

Erion DM \& Shulman GI. (2010). Diacylglycerol-mediated insulin resistance. Nature medicine $16,400-402$.

Farese RV, Jr. \& Walther TC. (2009). Lipid droplets finally get a little R-E-S-P-E-C-T. Cell 139, 855-860.

Fischer J, Lefevre C, Morava E, Mussini JM, Laforet P, Negre-Salvayre A, Lathrop M \& Salvayre R. (2007). The gene encoding adipose triglyceride lipase (PNPLA2) is mutated in neutral lipid storage disease with myopathy. Nat Genet 39, 28-30. 
Fox TE, Houck KL, O’Neill SM, Nagarajan M, Stover TC, Pomianowski PT, Unal O, Yun JK, Naides SJ \& Kester M. (2007). Ceramide recruits and activates protein kinase C zeta (PKC zeta) within structured membrane microdomains. The Journal of biological chemistry 282, 12450-12457.

Gemmink A, Bosma M, Kuijpers HJ, Hoeks J, Schaart G, van Zandvoort MA, Schrauwen P \& Hesselink MK. (2016). Decoration of intramyocellular lipid droplets with PLIN5 modulates fasting-induced insulin resistance and lipotoxicity in humans. Diabetologia 59, 1040-1048.

Ghanassia E, Brun JF, Fedou C, Raynaud E \& Mercier J. (2006). Substrate oxidation during exercise: type 2 diabetes is associated with a decrease in lipid oxidation and an earlier shift towards carbohydrate utilization. Diabetes Metab 32, 604-610.

Gjelstad IM, Haugen F, Gulseth HL, Norheim F, Jans A, Bakke SS, Raastad T, Tjonna AE, Wisloff U, Blaak EE, Riserus U, Gaster M, Roche HM, Birkeland KI \& Drevon CA. (2012). Expression of perilipins in human skeletal muscle in vitro and in vivo in relation to diet, exercise and energy balance. Arch Physiol Biochem 118, 22-30.

Goodpaster BH, He J, Watkins S \& Kelley DE. (2001). Skeletal muscle lipid content and insulin resistance: evidence for a paradox in endurance-trained athletes. The Journal of clinical endocrinology and metabolism 86, 5755-5761.

Gray RE, Tanner CJ, Pories WJ, MacDonald KG \& Houmard JA. (2003). Effect of weight loss on muscle lipid content in morbidly obese subjects. American journal of physiology Endocrinology and metabolism 284, E726-732.

Green JG, Johnson NA, Sachinwalla T, Cunningham CW, Thompson MW \& Stannard SR. (2010). Low-carbohydrate diet does not affect intramyocellular lipid concentration or insulin sensitivity in lean, physically fit men when protein intake is elevated. Metabolism-Clinical and Experimental 59, 1633-1641.

Guarente L. (2006). Sirtuins as potential targets for metabolic syndrome. Nature 444, 868-874.

Guo Z, Burguera B \& Jensen MD. (2000). Kinetics of intramuscular triglyceride fatty acids in exercising humans. J Appl Physiol (1985) 89, 2057-2064.

Haemmerle G, Lass A, Zimmermann R, Gorkiewicz G, Meyer C, Rozman J, Heldmaier G, Maier R, Theussl C, Eder S, Kratky D, Wagner EF, Klingenspor M, Hoefler G \& Zechner R. (2006). Defective lipolysis and altered energy metabolism in mice lacking adipose triglyceride lipase. Science 312, 734-737.

Haemmerle G, Moustafa T, Woelkart G, Buttner S, Schmidt A, van de Weijer T, Hesselink M, Jaeger D, Kienesberger PC, Zierler K, Schreiber R, Eichmann T, Kolb D, Kotzbeck P, Schweiger M, Kumari M, Eder S, Schoiswohl G, Wongsiriroj N, Pollak NM, Radner FP, Preiss-Landl K, Kolbe T, Rulicke T, Pieske B, Trauner M, Lass A, Zimmermann R, Hoefler G, Cinti S, Kershaw EE, Schrauwen P, Madeo F, Mayer B \& Zechner R. (2011). ATGL-mediated fat catabolism regulates cardiac mitochondrial function via PPAR-alpha and PGC-1. Nature medicine 17, 1076-1085.

Hannun YA, Loomis CR \& Bell RM. (1986). Protein kinase C activation in mixed micelles. Mechanistic implications of phospholipid, diacylglycerol, and calcium interdependencies. The Journal of biological chemistry 261, 7184-7190.

He J, Goodpaster BH \& Kelley DE. (2004). Effects of weight loss and physical activity on muscle lipid content and droplet size. Obes Res 12, 761-769.

He J, Watkins S \& Kelley DE. (2001). Skeletal muscle lipid content and oxidative enzyme activity in relation to muscle fiber type in type 2 diabetes and obesity. Diabetes 50 , 
817-823.

Helge JW, Stallknecht B, Drachmann T, Hellgren LI, Jimenez-Jimenez R, Andersen JL, Richelsen B \& Bruun JM. (2011). Improved glucose tolerance after intensive life style intervention occurs without changes in muscle ceramide or triacylglycerol in morbidly obese subjects. Acta physiologica 201, 357-364.

Hoeks J, Mensink M, Hesselink MK, Ekroos K \& Schrauwen P. (2012). Long- and mediumchain fatty acids induce insulin resistance to a similar extent in humans despite marked differences in muscle fat accumulation. The Journal of clinical endocrinology and metabolism 97, 208-216.

Hoeks J, van Herpen NA, Mensink M, Moonen-Kornips E, van Beurden D, Hesselink MKC \& Schrauwen P. (2010). Prolonged Fasting Identifies Skeletal Muscle Mitochondrial Dysfunction as Consequence Rather Than Cause of Human Insulin Resistance. Diabetes 59, 2117-2125.

Holloway GP, Han XX, Jain SS, Bonen A \& Chabowski A. (2014). Chronic muscle stimulation improves insulin sensitivity while increasing subcellular lipid droplets and reducing selected diacylglycerol and ceramide species in obese Zucker rats. Diabetologia 57, 832-840.

Hoppeler H. (1999). Skeletal muscle substrate metabolism. International journal of obesity and related metabolic disorders : journal of the International Association for the Study of Obesity 23 Suppl 3, S7-10.

Itani SI, Ruderman NB, Schmieder F \& Boden G. (2002). Lipid-induced insulin resistance in human muscle is associated with changes in diacylglycerol, protein kinase $\mathrm{C}$, and IkappaB-alpha. Diabetes 51, 2005-2011.

Jacob S, Machann J, Rett K, Brechtel K, Volk A, Renn W, Maerker E, Matthaei S, Schick F, Claussen CD \& Haring HU. (1999). Association of increased intramyocellular lipid content with insulin resistance in lean nondiabetic offspring of type 2 diabetic subjects. Diabetes 48, 1113-1119.

Jazet IM, Schaart G, Gastaldelli A, Ferrannini E, Hesselink MK, Schrauwen P, Romijn JA, Maassen JA, Pijl H, Ouwens DM \& Meinders AE. (2008). Loss of $50 \%$ of excess weight using a very low energy diet improves insulin-stimulated glucose disposal and skeletal muscle insulin signalling in obese insulin-treated type 2 diabetic patients. Diabetologia 51, 309-319.

Johnson ML, Distelmaier K, Lanza IR, Irving BA, Robinson MM, Konopka AR, Shulman GI \& Nair KS. (2016). Mechanism by Which Caloric Restriction Improves Insulin Sensitivity in Sedentary Obese Adults. Diabetes 65, 74-84.

Johnson NA, Stannard SR, Mehalski K, Trenell MI, Sachinwalla T, Thompson CH \& Thompson MW. (2003). Intramyocellular triacylglycerol in prolonged cycling with high- and low-carbohydrate availability. Journal of applied physiology 94, 1365-1372.

Jonker JT, de Mol P, de Vries ST, Widya RL, Hammer S, van Schinkel LD, van der Meer RW, Gans RO, Webb AG, Kan HE, de Koning EJ, Bilo HJ \& Lamb HJ. (2013). Exercise and type 2 diabetes mellitus: changes in tissue-specific fat distribution and cardiac function. Radiology 269, 434-442.

Kanaley JA, Shadid S, Sheehan MT, Guo Z \& Jensen MD. (2009). Relationship between plasma free fatty acid, intramyocellular triglycerides and long-chain acylcarnitines in resting humans. J Physiol-London 587, 5939-5950.

Kiens B, Alsted TJ \& Jeppesen J. (2011). Factors regulating fat oxidation in human skeletal 
muscle. Obes Rev 12, 852-858.

Kimmel AR, Brasaemle DL, McAndrews-Hill M, Sztalryd C \& Londos C. (2010). Adoption of PERILIPIN as a unifying nomenclature for the mammalian PAT-family of intracellular lipid storage droplet proteins. Journal of lipid research 51, 468-471.

Kitessa SM \& Abeywardena MY. (2016). Lipid-Induced Insulin Resistance in Skeletal Muscle: The Chase for the Culprit Goes from Total Intramuscular Fat to Lipid Intermediates, and Finally to Species of Lipid Intermediates. Nutrients 8.

Koves TR, Sparks LM, Kovalik JP, Mosedale M, Arumugam R, DeBalsi KL, Everingham K, Thorne L, Phielix E, Meex RC, Kien CL, Hesselink MK, Schrauwen P \& Muoio DM. (2013). PPARgamma coactivator-1alpha contributes to exercise-induced regulation of intramuscular lipid droplet programming in mice and humans. Journal of lipid research 54, 522-534.

Krahmer N, Guo Y, Farese RV, Jr. \& Walther TC. (2009). SnapShot: Lipid Droplets. Cell 139, 1024-1024 e1021.

Krssak M, Falk Petersen K, Dresner A, DiPietro L, Vogel SM, Rothman DL, Roden M \& Shulman GI. (1999). Intramyocellular lipid concentrations are correlated with insulin sensitivity in humans: a ${ }^{1} \mathrm{H}$ NMR spectroscopy study. Diabetologia 42, 113-116.

Krssak M, Petersen KF, Bergeron R, Price T, Laurent D, Rothman DL, Roden M \& Shulman GI. (2000). Intramuscular glycogen and intramyocellular lipid utilization during prolonged exercise and recovery in man: a $13 \mathrm{C}$ and ${ }^{1} \mathrm{H}$ nuclear magnetic resonance spectroscopy study. The Journal of clinical endocrinology and metabolism 85, 748754.

Kuerschner L, Moessinger C \& Thiele C. (2008). Imaging of lipid biosynthesis: how a neutral lipid enters lipid droplets. Traffic 9, 338-352.

Lally JS, Snook LA, Han XX, Chabowski A, Bonen A \& Holloway GP. (2012). Subcellular lipid droplet distribution in red and white muscles in the obese Zucker rat. Diabetologia $55,479-488$.

Lara-Castro C, Newcomer BR, Rowell J, Wallace P, Shaughnessy SM, Munoz AJ, Shiflett AM, Rigsby DY, Lawrence JC, Bohning DE, Buchthal S \& Garvey WT. (2008). Effects of short-term very low-calorie diet on intramyocellular lipid and insulin sensitivity in nondiabetic and type 2 diabetic subjects. Metabolism-Clinical and Experimental 57, $1-8$.

Larson-Meyer DE, Borkhsenious ON, Gullett JC, Russell RR, Devries MC, Smith SR \& Ravussin E. (2008). Effect of dietary fat on serum and intramyocellular lipids and running performance. Med Sci Sports Exerc 40, 892-902.

Larson-Meyer DE, Heilbronn LK, Redman LM, Newcomer BR, Frisard MI, Anton S, Smith SR, Alfonso A, Ravussin E \& team PC. (2006). Effect of calorie restriction with or without exercise on insulin sensitivity, beta-cell function, fat cell size, and ectopic lipid in overweight subjects. Diabetes Care 29, 1337-1344.

Larson-Meyer DE, Newcomer BR \& Hunter GR. (2002). Influence of endurance running and recovery diet on intramyocellular lipid content in women: a ${ }^{1} \mathrm{H}$ NMR study. American journal of physiology Endocrinology and metabolism 282, E95-E106.

Lass A, Zimmermann R, Haemmerle G, Riederer M, Schoiswohl G, Schweiger M, Kienesberger P, Strauss JG, Gorkiewicz G \& Zechner R. (2006). Adipose triglyceride lipase-mediated lipolysis of cellular fat stores is activated by CGI-58 and defective in ChanarinDorfman Syndrome. Cell metabolism 3, 309-319. 
Lee S, Boesch C, Kuk JL \& Arslanian S. (2013). Effects of an overnight intravenous lipid infusion on intramyocellular lipid content and insulin sensitivity in African-American versus Caucasian adolescents. Metabolism-Clinical and Experimental 62, 417-423.

Li Y, Lee S, Langleite T, Norheim F, Pourteymour S, Jensen J, Stadheim HK, Storås TH, Davanger S, Gulseth HL, Birkeland KI, Drevon CA \& Holen T. (2014). Subsarcolemmal lipid droplet responses to a combined endurance and strength exercise intervention. Physiol Rep 2.

Listenberger LL, Ostermeyer-Fay AG, Goldberg EB, Brown WJ \& Brown DA. (2007). Adipocyte differentiation-related protein reduces the lipid droplet association of adipose triglyceride lipase and slows triacylglycerol turnover. Journal of lipid research 48 , 2751-2761.

Machann J, Etzel M, Thamer C, Haring HU, Claussen CD, Fritsche A \& Schick F. (2011). Morning to evening changes of intramyocellular lipid content in dependence on nutrition and physical activity during one single day: a volume selective ${ }^{1} \mathrm{H}-\mathrm{MRS}$ study. MAGMA 24, 29-33.

Mason RR, Meex RC, Russell AP, Canny BJ \& Watt MJ. (2014). Cellular localization and associations of the major lipolytic proteins in human skeletal muscle at rest and during exercise. PloS one 9, e103062.

Meex RC, Schrauwen-Hinderling VB, Moonen-Kornips E, Schaart G, Mensink M, Phielix E, van de Weijer T, Sels JP, Schrauwen P \& Hesselink MK. (2010). Restoration of muscle mitochondrial function and metabolic flexibility in type 2 diabetes by exercise training is paralleled by increased myocellular fat storage and improved insulin sensitivity. Diabetes 59, 572-579.

Molleyres LP \& Rando RR. (1988). Structural studies on the diglyceride-mediated activation of protein kinase C. The Journal of biological chemistry 263, 14832-14838.

Montell E, Turini M, Marotta M, Roberts M, Noe V, Ciudad CJ, Mace K \& Gomez-Foix AM. (2001). DAG accumulation from saturated fatty acids desensitizes insulin stimulation of glucose uptake in muscle cells. American journal of physiology Endocrinology and metabolism 280, E229-237.

Mori T, Takai Y, Yu B, Takahashi J, Nishizuka Y \& Fujikura T. (1982). Specificity of the fatty acyl moieties of diacylglycerol for the activation of calcium-activated, phospholipiddependent protein kinase. Journal of biochemistry 91, 427-431.

Moro C, Galgani JE, Luu L, Pasarica M, Mairal A, Bajpeyi S, Schmitz G, Langin D, Liebisch G \& Smith SR. (2009). Influence of gender, obesity, and muscle lipase activity on intramyocellular lipids in sedentary individuals. The Journal of clinical endocrinology and metabolism 94, 3440-3447.

Murphy DJ \& Vance J. (1999). Mechanisms of lipid-body formation. Trends in biochemical sciences 24, 109-115.

Nielsen J, Mogensen M, Vind BF, Sahlin K, Hojlund K, Schroder HD \& Ortenblad N. (2010). Increased subsarcolemmal lipids in type 2 diabetes: effect of training on localization of lipids, mitochondria, and glycogen in sedentary human skeletal muscle. Am J Physiol Endocrinol Metab 298, E706-713.

Pan DA, Lillioja S, Kriketos AD, Milner MR, Baur LA, Bogardus C, Jenkins AB \& Storlien LH. (1997). Skeletal muscle triglyceride levels are inversely related to insulin action. Diabetes 46, 983-988.

Perreault L, Bergman BC, Hunerdosse DM \& Eckel RH. (2010). Altered intramuscular 
lipid metabolism relates to diminished insulin action in men, but not women, in progression to diabetes. Obesity 18, 2093-2100.

Peters SJ, Samjoo IA, Devries MC, Stevic I, Robertshaw HA \& Tarnopolsky MA. (2012). Perilipin family (PLIN) proteins in human skeletal muscle: the effect of sex, obesity, and endurance training. Appl Physiol Nutr Metab 37, 724-735.

Petersen KF, Dufour S, Befroy D, Garcia R \& Shulman GI. (2004). Impaired mitochondrial activity in the insulin-resistant offspring of patients with type 2 diabetes. $\mathrm{N}$ Engl J Med 350, 664-671.

Petersen KF, Dufour S, Morino K, Yoo PS, Cline GW \& Shulman GI. (2012). Reversal of muscle insulin resistance by weight reduction in young, lean, insulin-resistant offspring of parents with type 2 diabetes. Proceedings of the National Academy of Sciences of the United States of America 109, 8236-8240.

Phielix E, Meex R, Ouwens DM, Sparks L, Hoeks J, Schaart G, Moonen-Kornips E, Hesselink MK \& Schrauwen P. (2012). High oxidative capacity due to chronic exercise training attenuates lipid-induced insulin resistance. Diabetes 61, 2472-2478.

Phillips SM, Green HJ, Tarnopolsky MA, Heigenhauser GJ \& Grant SM. (1996). Progressive effect of endurance training on metabolic adaptations in working skeletal muscle. Am J Physiol 270, E265-272.

Ploegh HL. (2007). A lipid-based model for the creation of an escape hatch from the endoplasmic reticulum. Nature 448, 435-438.

Powell DJ, Hajduch E, Kular G \& Hundal HS. (2003). Ceramide disables 3-phosphoinositide binding to the pleckstrin homology domain of protein kinase B (PKB)/Akt by a PKCzeta-dependent mechanism. Molecular and cellular biology 23, 7794-7808.

Pruchnic R, Katsiaras A, He J, Kelley DE, Winters C \& Goodpaster BH. (2004). Exercise training increases intramyocellular lipid and oxidative capacity in older adults. American journal of physiology Endocrinology and metabolism 287, E857-862.

Rambold AS, Cohen S \& Lippincott-Schwartz J. (2015). Fatty acid trafficking in starved cells: regulation by lipid droplet lipolysis, autophagy, and mitochondrial fusion dynamics. Developmental cell 32, 678-692.

Rico-Sanz J, Moosavi M, Thomas EL, McCarthy J, Coutts GA, Saeed N \& Bell JD. (2000). In vivo evaluation of the effects of continuous exercise on skeletal muscle triglycerides in trained humans. Lipids 35, 1313-1318.

Sacchetti M, Saltin B, Olsen DB \& van Hall G. (2004). High triacylglycerol turnover rate in human skeletal muscle. J Physiol 561, 883-891.

Sakurai Y, Tamura Y, Takeno K, Kumashiro N, Sato F, Kakehi S, Ikeda S, Ogura Y, Saga N, Naito H, Katamoto S, Fujitani Y, Hirose T, Kawamori R \& Watada H. (2011). Determinants of intramyocellular lipid accumulation after dietary fat loading in non-obese men. J Diabetes Investig 2, 310-317.

Samjoo IA, Safdar A, Hamadeh MJ, Glover AW, Mocellin NJ, Santana J, Little JP, Steinberg GR, Raha S \& Tarnopolsky MA. (2013). Markers of skeletal muscle mitochondrial function and lipid accumulation are moderately associated with the homeostasis model assessment index of insulin resistance in obese men. Plos One 8, e66322.

Schenk S \& Horowitz JF. (2007). Acute exercise increases triglyceride synthesis in skeletal muscle and prevents fatty acid-induced insulin resistance. The Journal of clinical investigation 117, 1690-1698.

Schmitz-Peiffer C, Craig DL \& Biden TJ. (1999). Ceramide generation is sufficient to account 
for the inhibition of the insulin-stimulated PKB pathway in C2C12 skeletal muscle cells pretreated with palmitate. The Journal of biological chemistry 274, 24202-24210. Schrauwen-Hinderling VB, Kooi ME, Hesselink MK, Moonen-Kornips E, Schaart G, Mustard KJ, Hardie DG, Saris WH, Nicolay K \& Schrauwen P. (2005). Intramyocellular lipid content and molecular adaptations in response to a 1-week high-fat diet. Obesity research 13, 2088-2094.

Schrauwen-Hinderling VB, Schrauwen P, Hesselink MK, van Engelshoven JM, Nicolay K, Saris WH, Kessels AG \& Kooi ME. (2003a). The increase in intramyocellular lipid content is a very early response to training. The Journal of clinical endocrinology and metabolism 88, 1610-1616.

Schrauwen-Hinderling VB, van Loon LJ, Koopman R, Nicolay K, Saris WH \& Kooi ME. (2003b). Intramyocellular lipid content is increased after exercise in nonexercising human skeletal muscle. J Appl Physiol (1985) 95, 2328-2332.

Schweiger M, Lass A, Zimmermann R, Eichmann TO \& Zechner R. (2009). Neutral lipid storage disease: genetic disorders caused by mutations in adipose triglyceride lipase/ PNPLA2 or CGI-58/ABHD5. American journal of physiology Endocrinology and metabolism 297, E289-296.

Schweiger M, Schoiswohl G, Lass A, Radner FP, Haemmerle G, Malli R, Graier W, Cornaciu I, Oberer M, Salvayre R, Fischer J, Zechner R \& Zimmermann R. (2008). The C-terminal region of human adipose triglyceride lipase affects enzyme activity and lipid droplet binding. The Journal of biological chemistry 283, 17211-17220.

Serup AK, Alsted TJ, Jordy AB, Schjerling P, Holm C \& Kiens B. (2016). Partial Disruption of Lipolysis Increases Postexercise Insulin Sensitivity in Skeletal Muscle Despite Accumulation of DAG. Diabetes 65, 2932-2942.

Shaw CS, Jones DA \& Wagenmakers AJ. (2008). Network distribution of mitochondria and lipid droplets in human muscle fibres. Histochemistry and cell biology 129, 65-72.

Shaw CS, Shepherd SO, Wagenmakers AJ, Hansen D, Dendale P \& van Loon LJ. (2012). Prolonged exercise training increases intramuscular lipid content and perilipin 2 expression in type I muscle fibers of patients with type 2 diabetes. American journal of physiology Endocrinology and metabolism 303, E1158-1165.

Shepherd SO, Cocks M, Tipton KD, Ranasinghe AM, Barker TA, Burniston JG, Wagenmakers AJ \& Shaw CS. (2012). Preferential utilization of perilipin 2-associated intramuscular triglycerides during $1 \mathrm{~h}$ of moderate-intensity endurance-type exercise. Exp Physiol 97, 970-980.

Shepherd SO, Cocks M, Tipton KD, Ranasinghe AM, Barker TA, Burniston JG, Wagenmakers AJ \& Shaw CS. (2013). Sprint interval and traditional endurance training increase net intramuscular triglyceride breakdown and expression of perilipin 2 and 5. J Physiol 591, 657-675.

Skovbro M, Baranowski M, Skov-Jensen C, Flint A, Dela F, Gorski J \& Helge JW. (2008). Human skeletal muscle ceramide content is not a major factor in muscle insulin sensitivity. Diabetologia 51, 1253-1260.

Sogaard D, Ostergard T, Blachnio-Zabielska AU, Baranowski M, Vigelso AH, Andersen JL, Dela F \& Helge JW. (2016). Training Does Not Alter Muscle Ceramide and Diacylglycerol in Offsprings of Type 2 Diabetic Patients Despite Improved Insulin Sensitivity. Journal of diabetes research 2016, 2372741.

Solomon TP, Sistrun SN, Krishnan RK, Del Aguila LF, Marchetti CM, O'Carroll SM, O'Leary 
VB \& Kirwan JP. (2008). Exercise and diet enhance fat oxidation and reduce insulin resistance in older obese adults. Journal of applied physiology 104, 1313-1319.

Stannard SR, Thompson MW, Fairbairn K, Huard B, Sachinwalla T \& Thompson CH. (2002). Fasting for $72 \mathrm{~h}$ increases intramyocellular lipid content in nondiabetic, physically fit men. American journal of physiology Endocrinology and metabolism 283, E11851191.

Steffensen CH, Roepstorff C, Madsen M \& Kiens B. (2002). Myocellular triacylglycerol breakdown in females but not in males during exercise. American journal of physiology Endocrinology and metabolism 282, E634-642.

Stellingwerff T, Boon H, Jonkers RA, Senden JM, Spriet LL, Koopman R \& van Loon LJ. (2007). Significant intramyocellular lipid use during prolonged cycling in endurance-trained males as assessed by three different methodologies. American journal of physiology Endocrinology and metabolism 292, E1715-1723.

Stephens NA \& Sparks LM. (2015). Resistance to the beneficial effects of exercise in type 2 diabetes: are some individuals programmed to fail? The Journal of clinical endocrinology and metabolism 100, 43-52.

Straczkowski M, Kowalska I, Baranowski M, Nikolajuk A, Otziomek E, Zabielski P, Adamska A, Blachnio A, Gorski J \& Gorska M. (2007). Increased skeletal muscle ceramide level in men at risk of developing type 2 diabetes. Diabetologia 50, 2366-2373.

Straczkowski M, Kowalska I, Nikolajuk A, Dzienis-Straczkowska S, Kinalska I, Baranowski M, Zendzian-Piotrowska M, Brzezinska Z \& Gorski J. (2004). Relationship between insulin sensitivity and sphingomyelin signaling pathway in human skeletal muscle. Diabetes 53, 1215-1221.

Stratford S, Hoehn KL, Liu F \& Summers SA. (2004). Regulation of insulin action by ceramide: dual mechanisms linking ceramide accumulation to the inhibition of Akt/protein kinase B. The Journal of biological chemistry 279, 36608-36615.

Szendroedi J, Yoshimura T, Phielix E, Koliaki C, Marcucci M, Zhang D, Jelenik T, Muller J, Herder C, Nowotny P, Shulman GI \& Roden M. (2014). Role of diacylglycerol activation of PKCtheta in lipid-induced muscle insulin resistance in humans. Proceedings of the National Academy of Sciences of the United States of America 111, 9597-9602.

Tarnopolsky MA, Rennie CD, Robertshaw HA, Fedak-Tarnopolsky SN, Devries MC \& Hamadeh MJ. (2007). Influence of endurance exercise training and sex on intramyocellular lipid and mitochondrial ultrastructure, substrate use, and mitochondrial enzyme activity. Am J Physiol Regul Integr Comp Physiol 292, R1271-1278.

Thamer C, Machann J, Bachmann O, Haap M, Dahl D, Wietek B, Tschritter O, Niess A, Brechtel K, Fritsche A, Claussen C, Jacob S, Schick F, Haring HU \& Stumvoll M. (2003). Intramyocellular lipids: anthropometric determinants and relationships with maximal aerobic capacity and insulin sensitivity. The Journal of clinical endocrinology and metabolism 88, 1785-1791.

Thiam AR, Farese RV, Jr. \& Walther TC. (2013). The biophysics and cell biology of lipid droplets. Nature reviews Molecular cell biology 14, 775-786.

Thrush AB, Brindley DN, Chabowski A, Heigenhauser GJ \& Dyck DJ. (2009). Skeletal muscle lipogenic protein expression is not different between lean and obese individuals: a potential factor in ceramide accumulation. The Journal of clinical endocrinology and metabolism 94, 5053-5061. 
Timmers S, de Ligt M, Phielix E, van de Weijer T, Hansen J, Moonen-Kornips E, Schaart G, Kunz I, Hesselink MKC, Schrauwen-Hinderling VB \& Schrauwen P. (2016). Resveratrol as Add-on Therapy in Subjects With Well-Controlled Type 2 Diabetes: A Randomized Controlled Trial. Diabetes Care 39, 2211-2217.

Timmers S, Konings E, Bilet L, Houtkooper RH, van de Weijer T, Goossens GH, Hoeks J, van der Krieken S, Ryu D, Kersten S, Moonen-Kornips E, Hesselink MK, Kunz I, SchrauwenHinderling VB, Blaak EE, Auwerx J \& Schrauwen P. (2011). Calorie restriction-like effects of 30 days of resveratrol supplementation on energy metabolism and metabolic profile in obese humans. Cell metabolism 14, 612-622.

Toledo FG, Menshikova EV, Azuma K, Radikova Z, Kelley CA, Ritov VB \& Kelley DE. (2008). Mitochondrial capacity in skeletal muscle is not stimulated by weight loss despite increases in insulin action and decreases in intramyocellular lipid content. Diabetes 57, 987-994.

Tonks KT, Coster AC, Christopher MJ, Chaudhuri R, Xu A, Gagnon-Bartsch J, Chisholm DJ, James DE, Meikle PJ, Greenfield JR \& Samocha-Bonet D. (2016). Skeletal muscle and plasma lipidomic signatures of insulin resistance and overweight/obesity in humans. Obesity (Silver Spring) 24, 908-916.

Unger RH. (2003). Lipid overload and overflow: metabolic trauma and the metabolic syndrome. Trends Endocrin Met 14, 398-403.

van de Weijer T, Havekes B, Bilet L, Hoeks J, Sparks L, Bosma M, Paglialunga S, Jorgensen J, Janssen MC, Schaart G, Sauerwein H, Smeets JL, Wildberger J, Zechner R, SchrauwenHinderling VB, Hesselink MK \& Schrauwen P. (2013). Effects of bezafibrate treatment in a patient and a carrier with mutations in the PNPLA2 gene, causing neutral lipid storage disease with myopathy. Circulation research 112, e51-54.

van Hees AM, Jans A, Hul GB, Roche HM, Saris WH \& Blaak EE. (2011). Skeletal muscle fatty acid handling in insulin resistant men. Obesity 19, 1350-1359.

van Hees AM, Saris WH, Hul GB, Schaper NC, Timmerman BE, Lovegrove JA, Roche HM \& Blaak EE. (2010). Effects of dietary fat modification on skeletal muscle fatty acid handling in the metabolic syndrome. Int J Obes (Lond) 34, 859-870.

van Loon LJ, Koopman R, Manders R, van der Weegen W, van Kranenburg GP \& Keizer HA. (2004). Intramyocellular lipid content in type 2 diabetes patients compared with overweight sedentary men and highly trained endurance athletes. American journal of physiology Endocrinology and metabolism 287, E558-565.

van Loon LJ, Koopman R, Stegen JH, Wagenmakers AJ, Keizer HA \& Saris WH. (2003a). Intramyocellular lipids form an important substrate source during moderate intensity exercise in endurance-trained males in a fasted state. J Physiol 553, 611-625.

van Loon LJ, Schrauwen-Hinderling VB, Koopman R, Wagenmakers AJ, Hesselink MK, Schaart G, Kooi ME \& Saris WH. (2003b). Influence of prolonged endurance cycling and recovery diet on intramuscular triglyceride content in trained males. American journal of physiology Endocrinology and metabolism 285, E804-811.

Van Proeyen K, Szlufcik K, Nielens H, Deldicque L, Van Dyck R, Ramaekers M \& Hespel P. (2011). High-fat diet overrules the effects of training on fiber-specific intramyocellular lipid utilization during exercise. Journal of applied physiology 111, 108-116.

Vermathen P, Saillen P, Boss A, Zehnder M \& Boesch C. (2012). Skeletal muscle (1)H MRSI before and after prolonged exercise. I. muscle specific depletion of intramyocellular lipids. Magn Reson Med 68, 1357-1367. 
Vistisen B, Hellgren LI, Vadset T, Scheede-Bergdahl C, Helge JW, Dela F \& Stallknecht B. (2008). Effect of gender on lipid-induced insulin resistance in obese subjects. European journal of endocrinology 158, 61-68.

Waltermann M, Hinz A, Robenek H, Troyer D, Reichelt R, Malkus U, Galla HJ, Kalscheuer R, Stoveken T, von Landenberg P \& Steinbuchel A. (2005). Mechanism of lipid-body formation in prokaryotes: how bacteria fatten up. Molecular microbiology 55, 750763.

Walther TC \& Farese RV, Jr. (2009). The life of lipid droplets. Biochimica et biophysica acta 1791, 459-466.

Walther TC \& Farese RV, Jr. (2012). Lipid droplets and cellular lipid metabolism. Annual review of biochemistry $81,687-714$.

Wanner G, Formanek H \& Theimer RR. (1981). The ontogeny of lipid bodies (spherosomes) in plant cells : Ultrastructural evidence. Planta 151, 109-123.

White LJ, Ferguson MA, McCoy SC \& Kim H. (2003). Intramyocellular lipid changes in men and women during aerobic exercise: a (1)H-magnetic resonance spectroscopy study. The Journal of clinical endocrinology and metabolism 88, 5638-5643.

Wietek BM, Machann J, Mader I, Thamer C, Haring HU, Claussen CD, Stumvoll M \& Schick F. (2004). Muscle type dependent increase in intramyocellular lipids during prolonged fasting of human subjects: a proton MRS study. Horm Metab Res 36, 639-644.

Wolins NE, Quaynor BK, Skinner JR, Tzekov A, Croce MA, Gropler MC, Varma V, YaoBorengasser A, Rasouli N, Kern PA, Finck BN \& Bickel PE. (2006). OXPAT/PAT-1 is a PPAR-induced lipid droplet protein that promotes fatty acid utilization. Diabetes 55, 3418-3428.

Yamaguchi T, Matsushita S, Motojima K, Hirose F \& Osumi T. (2006). MLDP, a novel PAT family protein localized to lipid droplets and enriched in the heart, is regulated by peroxisome proliferator-activated receptor alpha. The Journal of biological chemistry 281, 14232-14240.

Yu J, Novgorodov SA, Chudakova D, Zhu H, Bielawska A, Bielawski J, Obeid LM, Kindy MS \& Gudz TI. (2007). JNK3 signaling pathway activates ceramide synthase leading to mitochondrial dysfunction. The Journal of biological chemistry 282, 25940-25949.

Zderic TW, Davidson CJ, Schenk S, Byerley LO \& Coyle EF. (2004). High-fat diet elevates resting intramuscular triglyceride concentration and whole body lipolysis during exercise. American journal of physiology Endocrinology and metabolism 286, E217225.

Zehnder M, Christ ER, Ith M, Acheson KJ, Pouteau E, Kreis R, Trepp R, Diem P, Boesch C \& Decombaz J. (2006). Intramyocellular lipid stores increase markedly in athletes after 1.5 days lipid supplementation and are utilized during exercise in proportion to their content. Eur J Appl Physiol 98, 341-354.

Zehnder M, Ith M, Kreis R, Saris W, Boutellier U \& Boesch C. (2005). Gender-specific usage of intramyocellular lipids and glycogen during exercise. Med Sci Sports Exerc 37, 1517-1524.

Zimmermann R, Strauss JG, Haemmerle G, Schoiswohl G, Birner-Gruenberger R, Riederer M, Lass A, Neuberger G, Eisenhaber F, Hermetter A \& Zechner R. (2004). Fat mobilization in adipose tissue is promoted by adipose triglyceride lipase. Science 306, 1383-1386.

Zweytick D, Athenstaedt K \& Daum G. (2000). Intracellular lipid particles of eukaryotic cells. Biochimica et biophysica acta 1469, 101-120. 
Diet, exercise and lipid droplet dynamics 


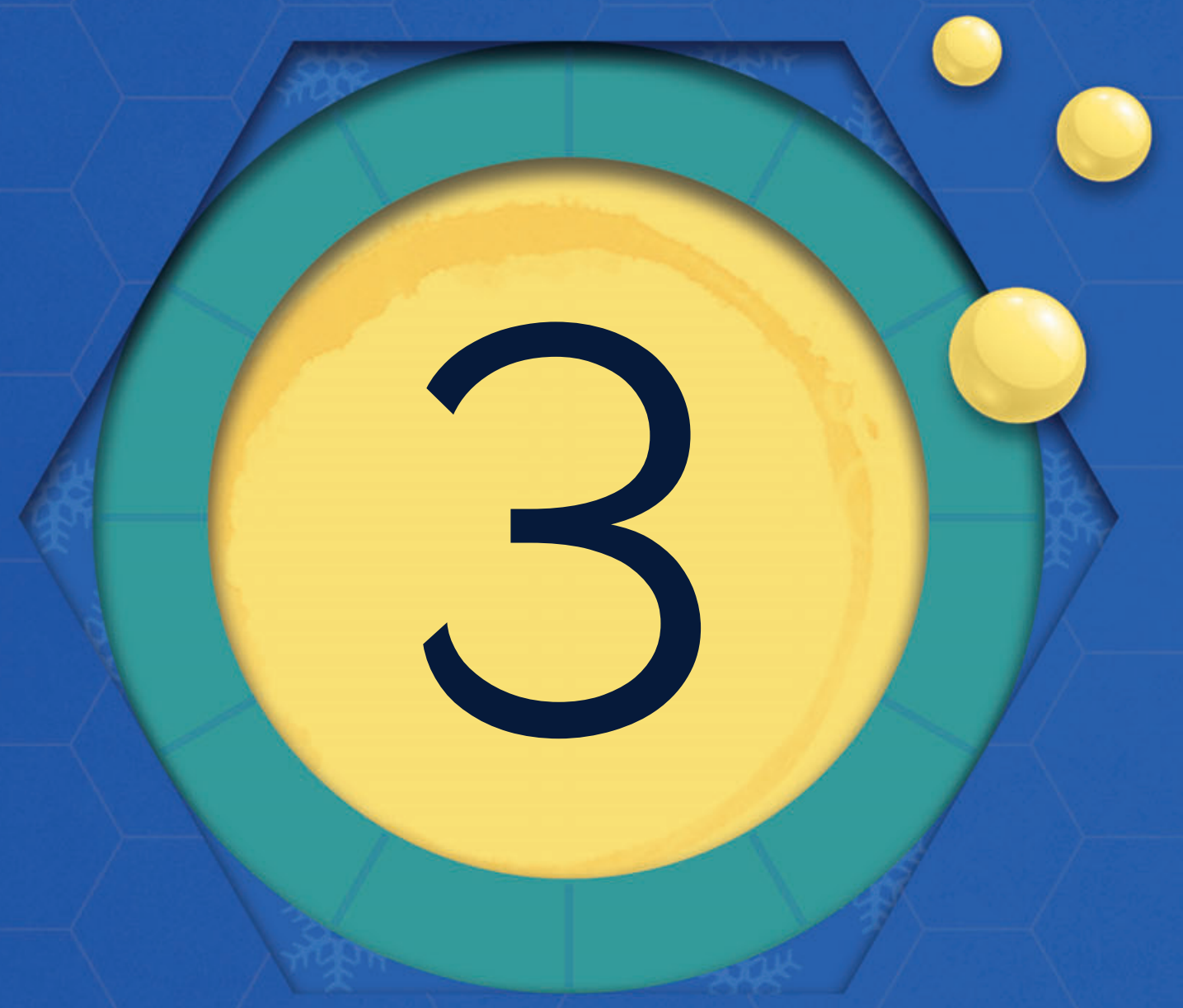

- 0 


\section{Acute cold exposure leads to increased levels of triglycerides during mixed-meal tests in young healthy subjects}

Michiel P.B. Moonen, Nynke van Polanen, Pascal Rense, Johanna A. Jörgensen, Esther Kornips, Maud Beckers, Joris Hoeks, Roel Wierts, Tineke van de Weijer, Bas Havekes, Jogchum Plat, Patrick Schrauwen, Wouter D. van Marken Lichtenbelt 


\section{Chapter 3}

\section{Abstract}

Atherosclerosis and elevated lipid levels are important factors contributing to the growing mortality and morbidity due to cardiovascular disease in the modern world. In order to reduce lipid levels, animal studies revealed that brown adipose tissue (BAT) is able to combust large amounts of circulating triglycerides. Here, we aim to investigate if stimulation of non-shivering thermogenesis via acute cold exposure could reduce lipid levels in humans. Fourteen healthy young volunteers, both male and female, were included. All volunteers underwent three separate mixed meal tests (lasting for 8 hours), under different thermal conditions: a) at thermoneutrality, b) during acute, non-shivering cold exposure and c) at thermoneutrality but after three hours of non-shivering, cold exposure (pre-cooling). Postprandial substrate and lipid metabolism were investigated by regular blood sampling, and skin temperatures and energy expenditure were measured. Muscle biopsies were obtained during acute cold exposure and at thermoneutrality to measure skeletal muscle mitochondrial function. On a separate day, cold-induced brown adipose tissue activity was measured using an ${ }^{18}$ FDG-PET/CT scan. Acute cold exposure increased resting and diet-induced energy expenditure $(17.4 \pm 3.4 \%$ and $10.5 \pm 1.2 \%)$ with a concomitant increase in fat oxidation $(8.6 \pm 1.6 \mathrm{~g}$ vs $5.1 \pm 1.0 \mathrm{~g} ; \mathrm{p}=0.079)$. Contrary to our hypothesis, postprandial triglyceride levels were increased during cold exposure compared to thermoneutral $(8.2 \pm 0.1 \mathrm{mmol} / 1 / \mathrm{min}$ vs $6.2 \pm 0.1 \mathrm{mmol} / 1 / \mathrm{min} ; \mathrm{p}=0.013)$, or compared to pre-cooling $(8.2 \pm 0.1 \mathrm{mmol} / 1 / \mathrm{min}$ vs $4.6 \pm 0.1 \mathrm{mmol} / 1 / \mathrm{min} ; \mathrm{p}=0.030)$. Acute cold exposure did not affect skeletal muscle mitochondrial function (maximum coupled respiration, $\mathrm{p}=0.150$; leak respiration, $\mathrm{p}=0.740$ ). BAT activity and volume did not correlate with postprandial changes in plasma lipid levels. Opposite to findings in animal studies, acute non-shivering thermogenesis does not decrease postprandial lipids levels in young healthy volunteers, despite increased whole-body energy expenditure and fat oxidation. 


\section{Introduction}

In modern society, cardiovascular disease (CVD) has become one of the foremost causes of death, with up to $31.4 \%$ of all deaths worldwide (WHO, 2018). One of the most important risk factors for CVD is hyperlipidaemia (Nordestgaard \& Varbo, 2014), which is classified as elevated plasma levels of cholesterol and/or triglycerides. Indeed, lifestyle and pharmacological therapies are aimed at lowering levels of lowdensity lipoprotein cholesterol, known as the lipoprotein that carries the major risk for CVD (Nordestgaard \& Varbo, 2014) and on the lowering of circulating triglyceride levels. Hypertriglyceridemia is also considered as an important risk factor for CVD (Nordestgaard et al., 2007), even independent of high levels of LDL (Hokanson \& Austin, 1996; Austin et al., 2000).

Preclinical research from the last decade has provided strong evidence that brown adipose tissue (BAT) is an interesting tissue in the clearance of circulating triglycerides. BAT has the unique feature of having a special mitochondrial protein, uncoupling protein 1 (UCP-1) (Cannon \& Nedergaard, 2004), which is used to uncouple the mitochondrial proton gradient from ATP production. Therefore, this mitochondrial uncoupling leads to futile use of substrates, with fatty acids as main substrate (Cannon \& Nedergaard, 2004), thereby producing heat. It has been shown that activation of BAT through cold increases the density of BAT tissue on CT scan images in humans (Baba et al., 2010; Ouellet et al., 2012) indicating that intracellular lipids are combusted. As a result, BAT activation would eventually lead to a depletion of the intracellular lipid stores, and circulatory lipids are needed to replenish BAT lipid stores. Indeed, it was shown in mice that active BAT might be able to take up a large amount of particles containing triglycerides upon cold exposure (Khedoe et al., 2015). It is suggested that activated BAT will first utilize intracellular lipid stores, and upon continued activation will start to increase uptake of fatty acids from lipoprotein particles to maintain the heat production. Indeed, in a recent study with transgenic mice overexpressing UCP1, it was shown that cold exposure increases lipoprotein lipase activity in BAT (Bartelt et al., 2011). Furthermore, it was shown that activation of brown fat in APOE*3-Leiden.CETP mice leads to reduced atherosclerosis (Berbee 


\section{Chapter 3}

et al., 2015). Additionally, in ApoE-/- and LDLR-/- mice, the effect of cold exposure in preventing atherosclerosis is absent (Dong et al., 2013). These results suggest that the clearance of fatty acids from lipoprotein particles by activated BAT plays an important role in atherosclerosis development in mice.

In humans, the exact role of BAT is still under debate. We and others have shown that BAT is functionally present in human adults and activated by acute cold and cold acclimation (van Marken Lichtenbelt et al., 2009; Virtanen et al., 2009; Hanssen et al., 2016) in both obese human subjects (Vijgen et al., 2011) and patients with type 2 diabetes (Hanssen et al., 2015). Next to the presence of active BAT in adult humans, there is evidence that human BAT is involved in cold-induced metabolism (Muzik et al., 2013; Weir et al., 2018). More recently, Chondronikolas et al (Chondronikola et al., 2016) confirmed that short-term cold exposure results in an increased density of BAT tissue suggesting the use of intracellular lipid droplets, and a subsequent activation of white adipose tissue lipolysis to refuel the BAT. Additionally, Blondin et al. have shown that, comparable to mice, BAT is involved in the uptake of dietary fatty acids (Blondin et al., 2017b). In their study, they used only a single meal and a predetermined temperature on each participant. This could mean that they missed a so-called second meal effect and interindividual differences in the response to cold exposure. However, Blondin et al also concluded that the net contribution of BAT to the clearance of dietary fatty acids is small. Indeed, relative to rodents, in humans the amount of BAT is small (Nedergaard et al., 2001; Cannon \& Nedergaard, 2004). Hence, in addition to BAT, skeletal muscle can play a role in cold-induced energy and substrate metabolism. Indeed, it has previously been shown that the uncoupling process in muscle mitochondria is related to an increase in non-shivering thermogenesis (Wijers et al., 2008; Wijers et al., 2011) and muscle tissue could therefore be important in the clearance of plasma triglycerides.

Therefore, the aim of the current study was to investigate if acute cold exposure in humans can impact postprandial substrate metabolism and enhance triglyceride clearance. 


\section{Methods}

\section{Volunteers}

Fourteen healthy young volunteers, nine males and five females, completed the study. Eligibility was evaluated via a screening including assessment of blood parameters, cardiac function (electrocardiography), anthropometric measurements and a questionnaire about lifestyle. Inclusion criteria were: 20-50 years of age, BMI $20-30 \mathrm{~kg} / \mathrm{m}^{2}$, a normal physically active lifestyle ( $<3$ hours exercise per week), nonsmoking for at least 6 months at inclusion, no alcohol use of $>2$ servings per day and stable dietary habits (no weight loss or gain of more than $5 \mathrm{~kg}$ in the past 3 months). No medication use was allowed, except for the use of any form of hormonal anticonception in female volunteers. Volunteers were excluded if they had any active metabolic or cardiovascular diseases, were pregnant or if they had a haemoglobin content below $8.4 \mathrm{mmol} / \mathrm{L}$. All data were collected between April 2018 and November 2019.

\section{Ethical approval}

The study was conducted according to the declaration of Helsinki and was approved by the Ethics Committee of the Maastricht University Medical Centre. The study was registered at https://trialregister.nl (NTR6573). All volunteers provided their written informed consent before screening.

\section{Study design}

The volunteers participated in three meal tests, each characterized by different thermal conditions: thermoneutral (at thermoneutrality), thermoneutral with three hours of preceding cold exposure (pre-cooling) and during acute cold exposure, as shown in Figure 1. On a separate day, all volunteers underwent an ${ }^{18} \mathrm{~F}-\mathrm{FDG}$ PET/ CT-scan directly after four hours of acute non-shivering cold exposure to assess the individual's capacity to activate BAT, as described previously (van der Lans et al., 


\section{Chapter 3}

2013). Between each test day (meal test or PET/CT-scan) there were at least two days to prevent any effect of the previous test on the next test day. All test days were randomized, as to prevent any effect of the order of tests on the measurements.

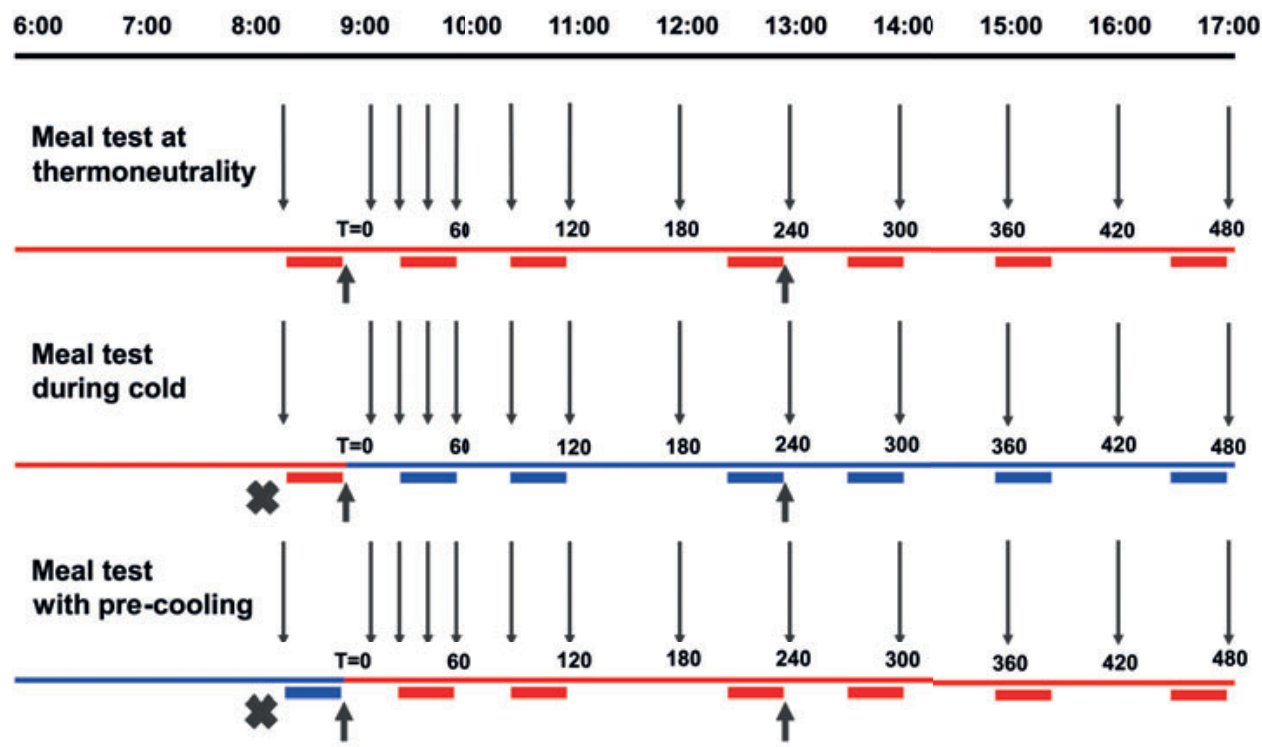

Figure 1. Study design. Overview of the three meal tests. The colour of horizontal lines indicates cooling (blue) or thermoneutral (red) conditions. Bold arrows indicate meal consumption. Crosses show muscle biopsies. Horizontal boxes indicate the indirect calorimetric measurements. Thin arrows show blood drawings.

\section{Cold exposure}

To investigate the effect of BAT activity on lipid metabolism, we cooled volunteers down to maximize non-shivering thermogenesis, as described before (van der Lans et al., 2013). In short, volunteers were wrapped in a water-perfused suit (ThermoWrap Universal 3166; MTRE Advanced Technologies Ltd., Yavne, Israel), while the water temperature was controlled using two temperature management units (Blanketrol M3; Cincinnati Sub Zero, OH, USA). First, volunteers remained at thermoneutral conditions $\left(32{ }^{\circ} \mathrm{C}\right.$ water temperature), after which the water in the suit was cooled in a stepwise fashion, $4{ }^{\circ} \mathrm{C}$ per 15 minutes. This was done until visible shivering occurred, at which point the subjects were heated to $34^{\circ} \mathrm{C}$ for 10 minutes to minimize shivering. After these 10 minutes, the water temperature was set back to $2{ }^{\circ} \mathrm{C}$ above the 
shivering onset temperature. For the remainder of each cooling period, temperature was increased by $1{ }^{\circ} \mathrm{C}$ if visible shivering occurred again.

\section{Meal test}

Volunteers arrived at our research centrum the evening before each meal test, around 6.00 PM. Upon arrival, they consumed a standardized meal, after which they were asked to remain fasted. The volunteers stayed overnight at the lab and were asked to sleep from 10.00 PM onwards. The following morning, volunteers were woken at $5.30 \mathrm{AM}$, after which they moved to a separate room and were asked to lie down on a bed in our research lab.

After placing an intravenous cannula, a fasted blood sample was drawn $(\mathrm{T}=0)$ and energy expenditure and substrate selection was measured by indirect calorimetry with a ventilated hood system (Omnical; Maastricht Instruments, Maastricht, the Netherlands). Between 6.00 AM and 9.00 AM, the volunteers were instructed to remain on the bed, and remain fasted. At 9.00 AM, volunteers were asked to consume a high-fat ( $\sim 60 \%$ fat) shake within ten minutes. The shake had a mean temperature of $43.5 \pm 0.8^{\circ} \mathrm{C}$, as measured with a thermocouple (PL-125-T4USB VS, Voltcraft, Conrad Electronic SE, Hirschau, Germany). The nutritional content of the shake is shown in supplementary Table S1. Subsequently, blood samples were drawn after 15, 30, 45, $60,90,120,180,240,300,360,420$ and 480 minutes. After the blood sampling at $\mathrm{T}=240$, at $1.00 \mathrm{PM}$, the volunteers consumed a second milkshake (with the same composition as in the morning) to investigate the so-called second-meal effect, as described before (Baumgartner et al., 2016). At T30-60, T90-120, T210-240, T270-300, T350-380 and T450480 indirect calorimetry was performed to measure energy expenditure and substrate oxidation by ventilated hood. Volunteers were not allowed to eat or drink anything else throughout the meal test, except for water.

The difference between the meal tests was the temperature to which the volunteers were exposed, as shown in Figure 1. The first meal test was performed at thermoneutrality. The second meal test was performed during cold exposure via the 


\section{Chapter 3}

water-perfused suit to maximize non-shivering thermogenesis during the meal test. The third meal test was performed after cold exposure; here volunteers were exposed to cold from 6.00 AM until 9.00 AM, again to maximize non-shivering thermogenesis (pre-cooling). After 9.00 AM, the volunteers were exposed to a thermoneutral temperature. The pre-cooling was meant to deplete the intracellular lipid storages of BAT tissue.

\section{${ }^{18}$ F-FDG PET/CT scan}

For the determination of cold-induced BAT activity, volunteers first remained at thermoneutral conditions $\left(32{ }^{\circ} \mathrm{C}\right.$ water) for 30 minutes, during which basal metabolic rate was measured with a ventilated hood system (Omnical). Thereafter an individualized cooling procedure was started to determine non-shivering thermogenesis (NST) as described above, after which energy expenditure was measured for 30 minutes. Following this, $75 \mathrm{MBq}$ of ${ }^{18} \mathrm{~F}-\mathrm{FDG}$ was injected via an intravenous cannula. Cold exposure was continued for 60 minutes, while volunteers were instructed to remain lying still. Next, the water-perfused suit was removed and the volunteers underwent a static ${ }^{18} \mathrm{~F}-\mathrm{FDG}$-PET/CT scan.

For the first five volunteers a Gemini TF PET-CT (Philips, the Netherlands) was used (120 kV, $30 \mathrm{mAs}$, six to seven bed position (5 minutes per bed position)) to cover the area from the skull to the iliac crest. For the other nine volunteers a Discovery MI 5R (GE Healthcare, Chicago, Ill, USA) was used (120 kV, $30 \mathrm{mAs}$, a maximum of four bed position was used (4 minutes per bed position)). A PET scan followed the lowdose CT scan. Tracer uptake was determined with the PET scan while the CT scan was used for attenuation correction and anatomical localization of the active BAT. With the use of an EARL reconstruction, the scans from the nine volunteers were made comparable to the scans from the five volunteers as described by Boellaard et al. (Boellaard et al., 2010). This ensured that we were able to compare results from both scanners in this study. 
The scans were analysed with PMOD software (version 3.0; PMOD Technologies). The regions of interest were manually outlined, while thresholds of $1.5 \mathrm{SUV}$ (standardized uptake value) and Hounsfield units between -10 and -180 were used, as described previously by our group (van der Lans et al., 2013). Additionally, fixed volumes $(10 \mathrm{~mm}$ by $10 \mathrm{~mm}$ ) were placed in the cervical adipose tissue behind the clavicle to measure general uptake values as described before (Vosselman et al., 2013). BAT activity was expressed in SUV $\left({ }^{18} \mathrm{~F}-\mathrm{FDG}\right.$ uptake $(\mathrm{kBq} / \mathrm{mL} /$ (injected dose $(\mathrm{kBq} /$ volunteer weight (g))))). The activity was determined as average SUV (SUVmean) and as total SUV (SUVmean times the volume of interest).

\section{Indirect calorimetry}

Whole-body oxygen consumption and carbon dioxide production were measured during fixed time points (Figure 1) in the meal tests with the use of an automated respiratory gas analyser with a ventilated hood system (Omnical). Volunteers were measured in supine position for 30 minutes each time. The measured averaged oxygen and carbon dioxide concentrations were used to calculate energy expenditure, glucose and fat oxidation with the assumption that protein oxidation was negligible (Weir, 1949; Péronnet \& Massicotte, 1991).

\section{Skin temperature}

During all measurements, volunteers were asked to wear shorts and t-shirt (overall clo value 0.49 ), and were instructed to remain supine and resting. Wireless temperature sensors (iButtons DS1922L, Maxim Integrated, San Jose, CA, USA) were placed on 15 ISO-defined sites (ISO-standard 9886:200) during all measurement days to measure skin temperature (van Marken Lichtenbelt et al., 2006). Average skin temperature, as well as proximal and distal temperature, was calculated as described before (Vosselman et al., 2015). 


\section{Chapter 3}

\section{Skeletal muscle biopsies and mitochondrial function}

To assess the effect of acute cold exposure on mitochondrial respiration, two muscle biopsies were taken from the $\mathrm{m}$. vastus lateralis under local anaesthesia using the Bergstrom technique (Bergström et al., 1967). These biopsies were taken after an overnight fast before the consumption of the first shake (Figure 1). One biopsy was taken during cold exposure (under stable non-shivering conditions) and one biopsy was taken under thermoneutral conditions. After the incision was made, a sterile golden temperature probe (BAT-10 Multipurpose Thermometer, Physitemp Instruments LLC, Clifton, NJ, USA) was inserted into the muscle to measure both muscle temperature and skin temperature. Subsequently, the biopsy was taken and the obtained muscle tissue was immediately placed in ice-cold preservation medium (BIOPS, OROBOROS Instruments, Innsbruck, Austria). Muscle fibres were permeabilized with saponin $(50 \mathrm{mg} / \mathrm{ml})$ and afterwards transferred into ice-cold mitochondrial respiration buffer (MiR05, OROBOROS Instruments, Innsbruck, Austria). Next, ex vivo mitochondrial function was determined by measuring oxygen consumption rate upon several substrates using high-resolution respirometry (Oxygraph, OROBOROS Instruments, Innsbruck, Austria) as described previously (Hoeks et al., 2010). All measurements were performed in triplicate or quadruplicate. As a quality measure, cytochrome c $(20 \mu \mathrm{M})$ was added in every individual experiment and the mitochondrial membrane was deemed intact if the response to the cytochrome c addition remained under $20 \%$. Oxygen consumption rate (flux) is expressed as pmol/(s*mg), corrected for wet muscle weight (2-3 mg).

\section{Blood sampling and analyses}

Blood collected in EDTA-coated tubes was immediately stored on ice, centrifuged and plasma was stored at $-80{ }^{\circ} \mathrm{C}$ until further analyses. Blood collected in serum tubes was stored at room temperature for at least 30 minutes to allow coagulation, followed by centrifugation and storage at -80 C until further analyses. Glucose (Hk$\mathrm{CP}$, Axonlab, Amsterdam, the Netherlands), triglycerides (Sigma, Zwijndrecht, the Netherlands) and free fatty acids (FFA) (NEFA-HR, WAKO chemicals, Neuss, 
Germany) were determined enzymatically in EDTA plasma derived from the meal test samples using a Pentra 400 (Horiba, Montpellier, France).

\section{Analysis of lipid particles via NMR spectroscopy}

Blood collected in serum-tubes, stored at $-80^{\circ} \mathrm{C}$ were analysed to specifically measure concentrations of lipoprotein particles using nuclear magnetic resonance (NMR) spectroscopy (Axinon Magnetic Group Analysis, Numares health, Regensburg Germany).

\section{Statistical analyses}

Subject characteristics are reported as mean \pm SD. Other results are reported as mean \pm

SEM. Data are presented for $\mathrm{n}=14$ unless otherwise indicated. All data were evaluated for normal distribution. Data of three different meal test days was compared using a total area-under-the-curve (AUC) and an incremental AUC (iAUC). The AUC and iAUC were calculated for the duration of the meal test, starting at $9.00 \mathrm{AM}$ and ending at 5.00 PM. Differences between meal tests were analysed with a repeated measures ANOVA for parametric data and with a Friedman's ANOVA for non-parametric data. Statistical significance was set at $\mathrm{p}<0.05$. Statistical analyses were performed using IBM SPSS version 26.0 and GraphPad Prism version 8.2.

\section{Results}

\section{Subject characteristics}

Fourteen healthy young volunteers (nine men, five women, age: $26 \pm 7$ years) participated in this study. Volunteers were non-obese (BMI: $23.3 \pm 1.6 \mathrm{~kg} / \mathrm{m}^{2}$ ), were non-smokers, did not use any medication except for hormonal anticonception in women and had an overall sedentary lifestyle (Baecke score 10.4 \pm 2.1 ). The participant characteristics are presented in Table 1 . There were no significant differences between male and female volunteers in BMI $(\mathrm{p}=0.417)$, age $(\mathrm{p}=0.310)$ and triglyceride levels at screening $(\mathrm{p}=0.771)$. 


\section{Chapter 3}

Table 1. Participant characteristics

\begin{tabular}{lc}
\hline Parameter & Mean \pm SD \\
\hline Number of volunteers (female/male) & $5 / 9$ \\
Age (years) & $26 \pm 7$ \\
Body weight $(\mathrm{kg})$ & $72.5 \pm 10.1$ \\
Height (m) & $1.75 \pm 0.10$ \\
BMI (kg/m $\left.{ }^{2}\right)$ & $23.3 \pm 1.6$ \\
Glucose (mmol/L) & $4.9 \pm 0.4$ \\
TG (mmol/L) & $1.05 \pm 0.60$ \\
ASAT (U/L) & $23 \pm 6$ \\
ALAT (U/L) & $24 \pm 9$ \\
GGT (U/L) & $20 \pm 7$ \\
eGFR (ml/min/1.73mm ${ }^{2}$ ) & $89 \pm 9$ \\
Physical activity level (Baecke score) & $10.4 \pm 2.1$ \\
\hline
\end{tabular}

Abbreviations: BMI, body mass index; TG, triglycerides; ASAT, aspartate aminotransferase; ALAT, alanine aminotransferase; GGT, gamma-glutamyl transferase; eGFR, estimated glomerular filtration rate according to the CKD-EPI method.

\section{Skin and muscle temperatures}

First, to investigate the body's response to cold exposure, we measured skin and muscle temperature. During the meal test, the average mean skin temperature was significantly higher at thermoneutrality compared to cold $\left(34.8 \pm 0.1^{\circ} \mathrm{C}\right.$ vs $31.3 \pm 0.5^{\circ} \mathrm{C}$, $\mathrm{p}<0.001)$, however, it was not significantly different compared to the meal test with pre-cooling $\left(33.6 \pm 0.7^{\circ} \mathrm{C}\right.$; $\mathrm{p}=0.156$, Figure 2A). There were no significant differences between genders in mean skin temperature during cold exposure $(p=0.999)$ or after pre-cooling $(\mathrm{p}=0.318)$. There was however a significant difference in mean skin temperature between male and female volunteers at thermoneutral conditions $\left(34.6 \pm 0.1{ }^{\circ} \mathrm{C}\right.$ vs $\left.35.0 \pm 0.1{ }^{\circ} \mathrm{C} ; \mathrm{p}=0.011\right)$ These results are shown supplemental Figure S2A. Mean proximal skin temperature was significantly higher during the meal test at thermoneutrality compared to cold $\left(35.7 \pm 0.04{ }^{\circ} \mathrm{C}\right.$ vs $\left.31.8 \pm 0.6^{\circ} \mathrm{C}, \mathrm{p}<0.001\right)$, however it was not significantly different compared to pre-cooling $\left(34.5 \pm 1.0^{\circ} \mathrm{C} ; \mathrm{p}=0.263\right.$ Figure 2B). There was a significant difference in proximal skin temperature between male and female volunteers during the meal test at thermoneutrality $\left(35.4 \pm 0.1{ }^{\circ} \mathrm{C}\right.$ vs $\left.36.0 \pm 0.1^{\circ} \mathrm{C} ; \mathrm{p}<0.001\right)$ There were no gender differences in the meal test during cold 
$(\mathrm{p}=0.383)$ or with pre-cooling $(\mathrm{p}=0.259)$, as shown in Figure S2B. Similarly, the mean distal temperature was significantly higher during the meal test at thermoneutrality compared to cold $\left(33.4 \pm 0.2^{\circ} \mathrm{C}\right.$ vs $\left.28.7 \pm 0.7^{\circ} \mathrm{C}, \mathrm{p}<0.001\right)$, and it showed a trend towards a significant difference compared to pre-cooling $\left(31.3 \pm 0.8^{\circ} \mathrm{C}\right.$; $\mathrm{p}=0.070$, Figure $\left.2 \mathrm{C}\right)$. There were no gender differences in distal skin temperature during the meal tests ( $\mathrm{p}=0.259$ at thermoneutrality; $\mathrm{p}=0.902$ during cold; $\mathrm{p}=0.383$ with pre-cooling) as shown in Figure S2C.

The mean proximal-distal gradient did not differ significantly between the meal test at thermoneutrality compared to cold $\left(2.4 \pm 0.2^{\circ} \mathrm{C}\right.$ vs $\left.3.0 \pm 0.6{ }^{\circ} \mathrm{C}, \mathrm{p}=0.169\right)$ or precooling $\left(3.2 \pm 0.8{ }^{\circ} \mathrm{C}, \mathrm{p}=0.302\right.$, Figure $\left.2 \mathrm{D}\right)$. There were no gender differences in the meal tests at thermoneutrality, during cold or after pre-cooling $(\mathrm{p}=0.456 ; \mathrm{p}=0.318$; $\mathrm{p}=0.999)$, as shown in Figure S2D. Regarding the mean underarm-finger gradient, we did observe a significantly lower gradient during the meal test at thermoneutrality compared to the meal test during cold $\left(-0.8 \pm 0.1{ }^{\circ} \mathrm{C}\right.$ vs $\left.5.7 \pm 0.9{ }^{\circ} \mathrm{C}, \mathrm{p}<0.001\right)$. The mean underarm-finger gradient was also higher in the meal test during cold exposure compared to the meal test with pre-cooling $\left(1.2 \pm 0.9^{\circ} \mathrm{C}, \mathrm{p}=0.044\right.$, Figure 2E). During the meal test at thermoneutrality, there was a lower gradient in the female volunteers compared to men $\left(-1.7 \pm 0.2{ }^{\circ} \mathrm{C}\right.$ vs $\left.-0.2 \pm 0.1{ }^{\circ} \mathrm{C} ; \mathrm{p}<0.001\right)$. However, there were no gender differences in the meal tests during cold $(\mathrm{p}=0.383)$ or after pre-cooling $(\mathrm{p}=0.318)$ as shown in Figure S2E.

Muscle temperature was significantly higher during thermoneutral condition compared to cold exposure $\left(35.1 \pm 0.2^{\circ} \mathrm{C}\right.$ vs $31.9 \pm 0.4{ }^{\circ} \mathrm{C}$; $\left.\mathrm{p}<0.001\right)$. 

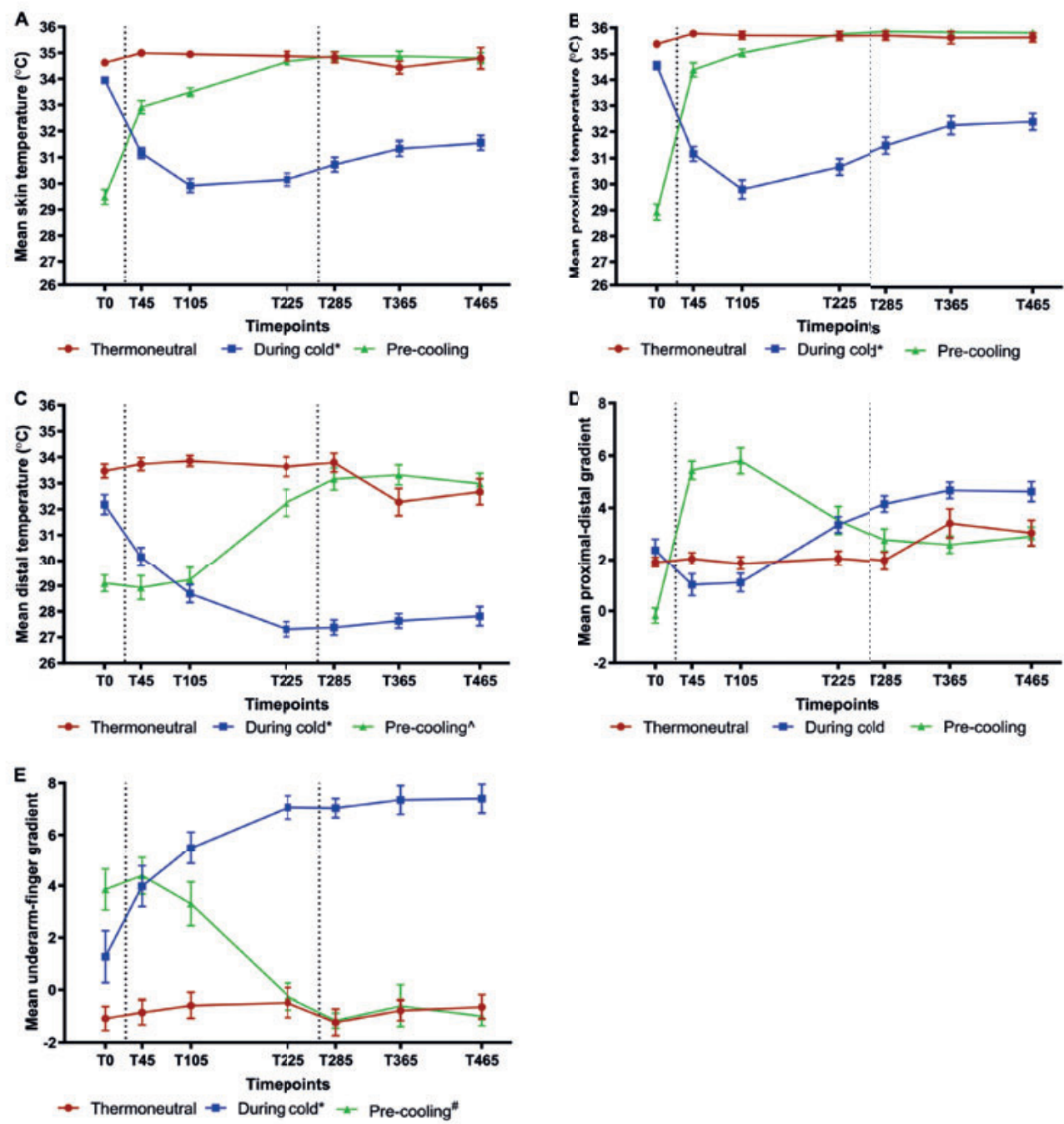

Figure 2. Skin temperatures during the meal tests. A) Mean skin temperature during the different meal tests. B) Mean proximal skin temperature. C) Mean distal skin temperature, D) Mean proximal-distal gradient and E) Mean underarm-finger gradient. The meal test at thermoneutrality is indicated as the red line, the meal test during cold exposure as the blue line, and the meal test after cold as the green line. Dashed vertical lines show the time of consumption of the 1st shake at TO and the 2nd shake at T240. Data is presented as mean \pm SEM. $(n=14)$ * indicates significant different from thermoneutral $(p<0.05)$. \# indicates significant different from cold $(p<0.05) .{ }^{\wedge}$ indicates trends towards significant different from thermoneutral $(p<0.10)$ 


\section{Postprandial energy and substrate metabolism}

Next, we examined if cold exposure had an effect on energy expenditure during the meal test. Total energy expenditure during the meal test, calculated as AUC, was significantly higher during cold exposure compared to thermoneutrality $(2772 \pm 130.3$ $\mathrm{kJ}$ vs $2613 \pm 166.2 \mathrm{~kJ} ; \mathrm{p}=0.020)$ and pre-cooling $(2772 \pm 130.3 \mathrm{~kJ}$ vs $2587 \pm 110.6 \mathrm{~kJ} ; \mathrm{p}=0.005)$ (Figure 3A). However, this was only seen in the male volunteers $(\mathrm{p}=0.015)$, not in female volunteers $(p=0.540)$. Similar results were found when the iAUC values were calculated, with significantly higher energy expenditure values during cold exposure compared to thermoneutrality $(481.2 \pm 55.8 \mathrm{~kJ}$ vs $338.2 \pm 27.5 \mathrm{~kJ} ; \mathrm{p}=0.003)$ and cold exposure compared to pre-cooling $(481.2 \pm 55.8 \mathrm{~kJ}$ vs $302.3 \pm 36.6 \mathrm{~kJ}$; $\mathrm{p}=0.049)$ (Figure $3 \mathrm{~A})$. Again, these differences were present in male volunteers $(\mathrm{p}=0.014)$, however absent in female volunteers $(p=0.209)$. Results for male and female volunteers are shown in Figure S3A.

To calculate diet-induced thermogenesis (DIT), we subtracted average energy expenditure at rest from energy expenditure during the meal. DIT, expressed as a percentage of resting energy expenditure, was $10.5 \pm 1.2 \%$ at thermoneutrality. During cold exposure, the percentage increase in energy expenditure was $17.4 \pm 3.4 \%$, consisting of both DIT and cold-induced thermogenesis (CIT). These data suggest that the difference between DIT and CIT+DIT was $159 \pm 64 \mathrm{~kJ}$ or $6.1 \pm 2.3 \%$ which can be attributed to CIT and suggests additive effects of CIT and DIT.

For male volunteers, average DIT was $10.8 \pm 1.7 \%$, and average DIT+CIT was $19.5 \pm 4.7 \%$. For female volunteers, DIT was $10.1 \pm 2.0 \%$ and DIT+CIT was $13.7 \pm 4.8 \%$. This suggests that while DIT\% is similar in both male and female volunteers, there is a higher, though non-significant increase in energy expenditure in cold in men compared to women $(8.7 \pm 3.2 \%$ vs $3.6 \pm 3.4 \%$; $\mathrm{p}=0.52)$. 

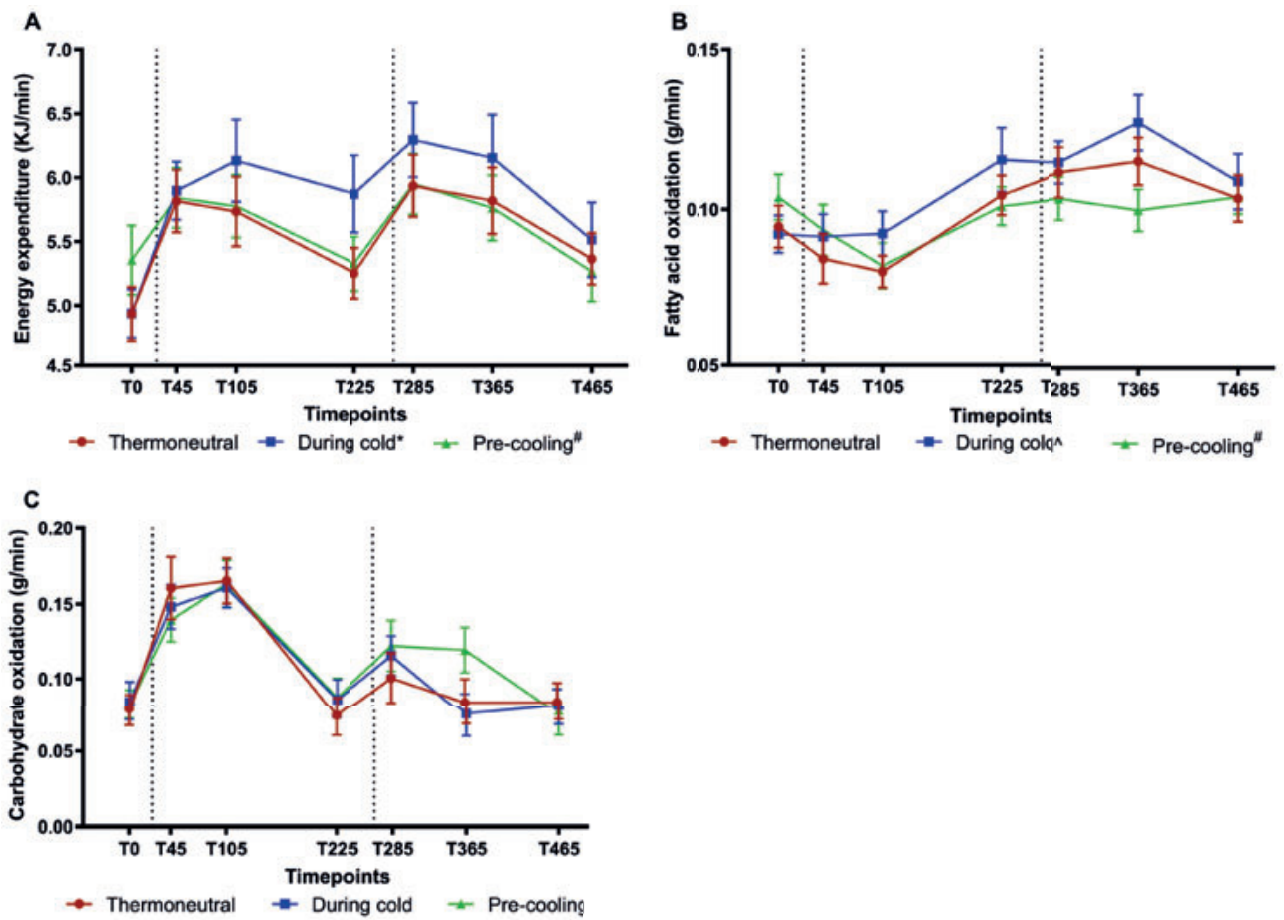

Figure 3. Energy expenditure and substrate oxidation during the meal tests. Energy expenditure (A), fatty acid oxidation (B) and carbohydrate oxidation (C) during the meal tests. The meal test at thermoneutrality is indicated as the red line, the meal test during cold exposure as the blue line and the meal test after cold as the green line. Dashed vertical lines indicate the time of consumption of the 1st shake at T0 and the 2nd shake at T240. Data is presented as mean \pm SEM $(n=14)$. * indicates significant different from thermoneutral $(p<0.05)$. \# indicates significant different from cold $(p<0.05) . \wedge$ indicates trends towards significant different from thermoneutral $(p<0.10)$.

The increase in energy expenditure during cold exposure was mainly accommodated by an increase in fat oxidation. The iAUC of fatty acid oxidation upon the meal test was significantly higher during cold exposure compared to pre-cooling $(8.6 \pm 1.6 \mathrm{~g}$ vs $2.1 \pm 0.7 \mathrm{~g} ; \mathrm{p}=0.002)$ and tended to be higher compared to thermoneutrality $(8.6 \pm 1.6 \mathrm{~g}$ vs 5.1 $\pm 1.0 \mathrm{~g} ; \mathrm{p}=0.079$ ), as shown in Figure 3B. For male volunteers, these differences were also present, as fatty acid oxidation was higher during cold compared to at thermoneutrality $(11.1 \pm 2.1 \mathrm{~g}$ vs $5.4 \pm 1.3 \mathrm{~g} ; \mathrm{p}=0.055)$ and compared to after pre-cooling $(2.2 \pm 1.0 \mathrm{~g} ; \mathrm{p}=0.012)$. In the female subjects, these differences were absent $(\mathrm{p}=0.315)$, as is shown in Figure S3B. No significant differences in iAUC of carbohydrate oxidation were observed between the different meal tests, as shown in Figure 3C, nor in males $(p=0.701)$ or females $(p=0.986)$ separately, as shown in Figure S3C. 


\section{Activity of brown adipose tissue via ${ }^{18}$ F-FDG PET/CT}

To investigate if the interindividual differences in energy expenditure and substrate oxidation to cold exposure were related to the individual's BAT activity, the latter was assessed using ${ }^{18} \mathrm{~F}-\mathrm{FDG}$-PET/CT after four hours of acute non-shivering cold exposure. On average, BAT volume was $66.8 \pm 12.4 \mathrm{~mL}$, with an average activity of $3.2 \pm 0.3 \mathrm{SUV}$ and a maximal activity of $8.9 \pm 1.2 \mathrm{SUV}$. The average BAT activity showed a trend towards significant positive correlation with CIT ( $r=0.46 ; p=0.097)$. However, there was no significant correlation between DIT and average BAT activity $(r=0.09$, $\mathrm{p}=0.739)$, or between fatty acid oxidation and average BAT activity $(r=0.34, p=0.234)$.

\section{Postprandial substrate kinetics}

Postprandial AUCs for triglycerides were not significantly different between the meal tests at thermoneutrality, during cold or with pre-cooling (respectively: $1.6 \pm 0.2$ $\mathrm{mmol} / 1 / \mathrm{min}$ vs $1.8 \pm 0.2 \mathrm{mmol} / 1 / \mathrm{min}$ vs $1.7 \pm 0.2 \mathrm{mmol} / 1 / \mathrm{min} ; \mathrm{p}=0.315$, Figure $4 \mathrm{~A}$ ). Remarkably and contrary to our hypothesis, the iAUC values for triglycerides were significantly higher during cold exposure compared to thermoneutrality $(0.82 \pm 0.1$ $\mathrm{mmol} / \mathrm{l} / \mathrm{min}$ vs $0.62 \pm 0.1 \mathrm{mmol} / \mathrm{l} / \mathrm{min} ; \mathrm{p}=0.013)$ or to pre-cooling $(0.82 \pm 0.1 \mathrm{mmol} / \mathrm{l} / \mathrm{min}$ vs $0.46 \pm 0.1 \mathrm{mmol} / 1 / \mathrm{min} ; \mathrm{p}=0.030$ ).

Similar trends were seen in both the male and female populations with higher iAUC values during cold compared to thermoneutrality albeit not statistically significant (male: $1.4 \pm 0.2 \mathrm{mmol} / \mathrm{l} / \mathrm{min}$ vs $0.8 \pm 0.1 \mathrm{mmol} / \mathrm{l} / \mathrm{min}$; $\mathrm{p}=0.098$ ) (female: $0.4 \pm 0.03 \mathrm{mmol} / \mathrm{l} /$ $\min$ vs $0.2 \pm 0.1 \mathrm{mmol} / 1 / \mathrm{min} ; \mathrm{p}=0.063$ ), as shown in Figure S4A.

Postprandial AUC of plasma FFA was significantly higher during cold exposure compared to thermoneutrality $(550.2 \pm 30.1 \mu \mathrm{mol} / 1 / \mathrm{min}$ vs $392.7 \pm 25.5 \mu \mathrm{mol} / \mathrm{l} / \mathrm{min}$; $\mathrm{p}<0.001)$ and compared to pre-cooling $(550.2 \pm 30.1 \mu \mathrm{mol} / \mathrm{l} / \mathrm{min}$ vs $433.9 \pm 35.2 \mu \mathrm{mol} / \mathrm{l} /$ min; $\mathrm{p}=0.005)$. These effects were also observed in male volunteers, with higher AUC of FFA during cold compared to at thermoneutrality $(552.4 \pm 36.8 \mu \mathrm{mol} / \mathrm{l} / \mathrm{min}$ vs $413.2 \pm 33.1 \mu \mathrm{mol} / \mathrm{l} / \mathrm{min} ; \mathrm{p}=0.004)$ and compared to pre-cooling $(454.1 \pm 50.4 \mu \mathrm{mol} / \mathrm{l} /$ min; $p=0.055)$. These effects were also present in female volunteers, with higher 


\section{Chapter 3}

FFA values during cold compared to thermoneutrality $(546.3 \pm 61.9 \mu \mathrm{mol} / 1 / \mathrm{min}$ vs $355.7 \pm 38.2 \mu \mathrm{mol} / \mathrm{l} / \mathrm{min} ; \mathrm{p}=0.063$ ), as shown in Figure S4B.

Similar effects were observed for FFA iAUC, which was significantly higher during the meal test in the cold compared to thermoneutrality $(278.0 \pm 28 \mu \mathrm{mol} / 1 / \mathrm{min}$ vs $235.4 \pm 19.8 \mu \mathrm{mol} / \mathrm{l} / \mathrm{min}$; $\mathrm{p}=0.042)$ or pre-cooling $(278.0 \pm 28 \mu \mathrm{mol} / \mathrm{l} / \mathrm{min}$ vs $172.8 \pm 17.3$ $\mu \mathrm{mol} / \mathrm{l} / \mathrm{min}$; $\mathrm{p}=0.002)$, as shown in Figure $4 \mathrm{~B}$. For males, higher iAUC values were observed during cold compared to at thermoneutrality $(280.9 \pm 29.0 \mu \mathrm{mol} / 1 / \mathrm{min}$ vs $233.4 \pm 21.0 \mu \mathrm{mol} / \mathrm{l} / \mathrm{min} ; \mathrm{p}=0.055)$ and compared to pre-cooling $(151.7 \pm 16.1 \mu \mathrm{mol} / 1 / \mathrm{min}$; $\mathrm{p}=0.004)$. These effects were absent in female volunteers, as shown in Figure S4B.

Postprandial AUC of plasma glucose was significantly lower during cold exposure compared to thermoneutrality $(5.3 \pm 0.1 \mu \mathrm{mol} / \mathrm{l} / \mathrm{min}$ vs $5.8 \pm 0.1 \mu \mathrm{mol} / \mathrm{l} / \mathrm{min} ; \mathrm{p}=0.007)$ and pre-cooling $(5.8 \pm 0.1 \mu \mathrm{mol} / \mathrm{l} / \mathrm{min}$ vs $5.9 \pm 0.1 \mu \mathrm{mol} / \mathrm{l} / \mathrm{min}$; $\mathrm{p}=0.005$; Figure $4 \mathrm{C})$. For both male $(\mathrm{p}=0.072)$ and female volunteers $(\mathrm{p}=0.057)$ there was a tendency towards significant differences (Figure S4C). 
A

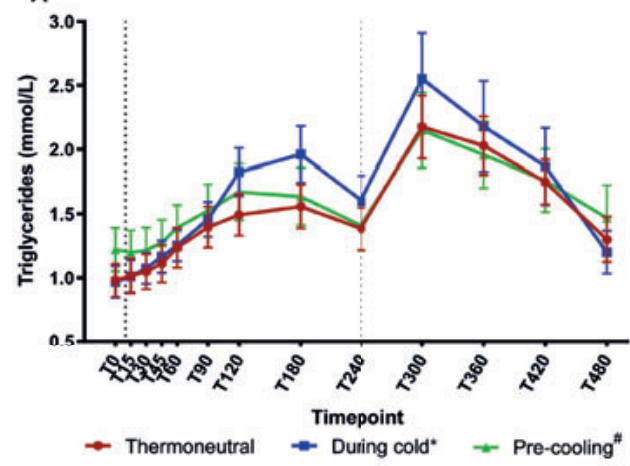

c

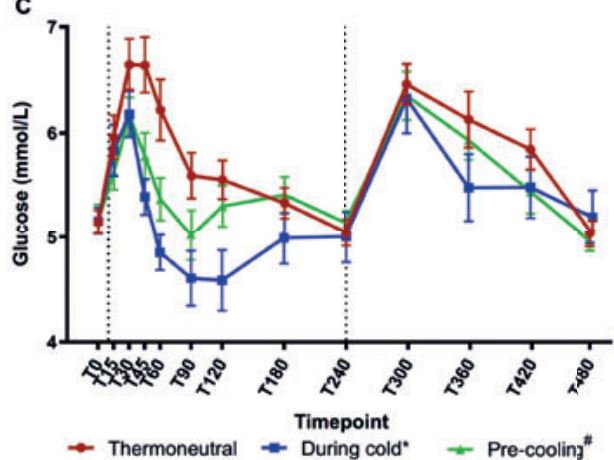

B

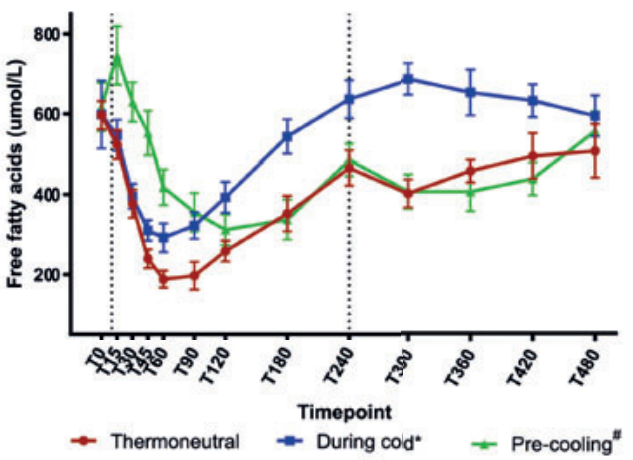

Figure 4. Plasma levels during the meal tests. Plasma levels of triglycerides (A), free fatty acids (B) and glucose (C) during the meal tests. The meal test at thermoneutrality is represented as the red line, the meal test during cold as the blue line and the meal test after cold as the green line. Dashed vertical lines indicate the time of consumption of the 1st shake at TO and the 2nd shake at T240. Data is presented as mean \pm SEM $(n=14) .{ }^{*}$ indicates significant different from thermoneutral $(p<0.05)$. \# indicates significant different from cold $(p<0.05)$. 


\section{Chapter 3}

\section{Mitochondrial function}

To investigate if acute cold exposure affects skeletal muscle mitochondrial function, muscle biopsies were taken during acute cold exposure and under thermoneutral conditions. No significant differences were observed in state 2 respiration (malate + octanoylcarnitine $(\mathrm{MO}), \mathrm{p}=0.776)$ or in ADP-stimulated state 3 respiration $(\mathrm{p}=0.345)$ (Figure 5A). Addition of glutamate (MOG3, $\mathrm{p}=0.369$; Figure 5A); and succinate (MOGS3, $\mathrm{p}=0.428$; Figure 5B) did not lead to a difference between the cold and thermoneutral conditions. Next, maximal uncoupled respiration, measured using FCCP titration, was not significantly different between the two conditions $(\mathrm{p}=0.150$; Figure 5B).

Mitochondrial reserve capacity, as defined by state $3 \mathrm{U} /$ state 3 was significantly higher thermoneutrality compared to cold exposure ( $1.26 \pm 0.02$ vs $1.20 \pm 0.02 ; \mathrm{p}=0.006)$, reflecting that under cold exposure, the skeletal muscle mitochondria have less reserve respiration. To investigate leak respiration, we performed a second trace in which we determined ADP-stimulated state 3 respiration upon malate + pyruvate and leak respiration upon addition of oligomycin (state 4o). No significant differences were observed between thermoneutral and cold conditions for state $3(p=0.939)$ or state 40 respiration $(\mathrm{p}=0.740)$ as shown in Figure $5 \mathrm{C}$. 
A

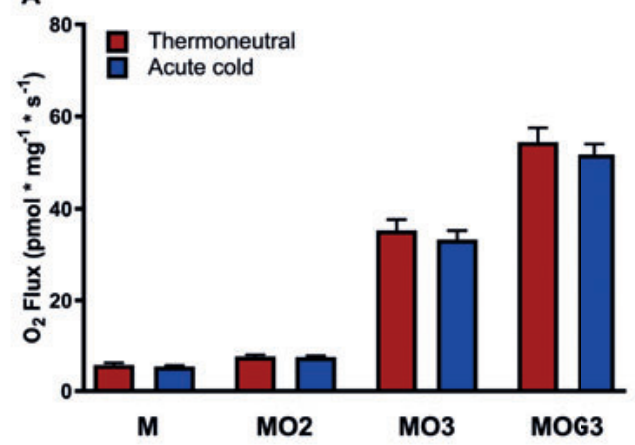

C

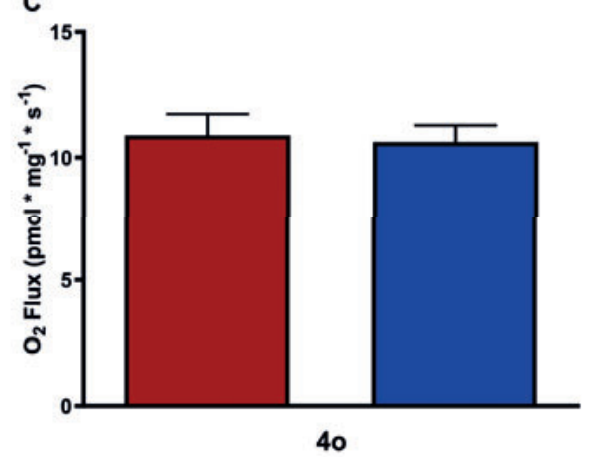

B

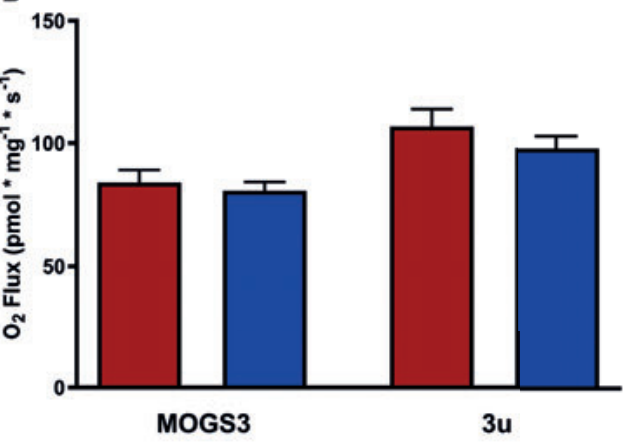

Figure 5. Mitochondrial function did not change upon acute cold exposure. A) Mitochondrial respiration upon malate $(M)$, state 2 respiration upon malate + octanoyl-carnitine (MO2), state 3 respiration after addition of ADP (MO3) and glutamate (MOG3) between thermoneutral and acute cold exposure. B) Maximally coupled respiration after addition of succinate (MOGS3) and FCCP-induced uncoupled (3u) respiration. C) Leak respiration upon oligomycin (state 4o). Data is presented as mean $\pm S E M(n=14$ in $A$ and $B, n=12$ in $C)$. 


\section{Chapter 3}

\section{Discussion}

Hyperlipidaemia is known to be one of the most important risk factors for developing cardiovascular disease (Nordestgaard \& Varbo, 2014). Reducing hyperlipidaemia could therefore decrease cardiovascular morbidity and mortality. There are indications from pre-clinical models that cold-induced activation of BAT leads to an increase in the clearance of plasma triglycerides (Bartelt et al., 2011). Therefore, in this study we aimed at investigating the effects of acute non-shivering cold exposure on postprandial lipidaemia in young healthy subjects.

Previous studies indicate potential metabolic benefits of cold exposure in humans, via activation of brown adipose tissue and/or skeletal muscle. Cold exposure, both acute and chronic exposure, has been shown to increase energy expenditure (van Marken Lichtenbelt et al., 2009; van der Lans et al., 2013). In addition, cold acclimation has shown to improve glucose metabolism in patients with diabetes (Hanssen et al., 2015). In our study, we observed a mean DIT of $10.5 \pm 1.2 \%$. Interestingly the percentual increase in postprandial energy expenditure during cold exposure, reflecting a combination of CIT and DIT, was $17.4 \pm 3.4 \%$. This suggests an additive effect of cold and food intake on energy expenditure in humans, as described before by others (Dauncey, 1980). It has previously been shown that under fasting conditions, CIT without shivering ranges from 0-30 \% (van Marken Lichtenbelt \& Schrauwen, 2011). The present study shows that cold exposure increased energy expenditure during the meal test on top of DIT.

The increase in energy expenditure in the present study was mainly accounted for by an increase in fat oxidation, as was also found previously upon cold exposure (Vallerand \& Jacobs, 1989). Interestingly this increase in energy expenditure during cold exposure was less pronounced in the female volunteers, though not statistically significant. This could indicate that in women there is less additional energy production needed to compensate for the cold exposure. As can be seen from the temperature results, there were no differences between male and female volunteers during the cold exposure. 
Preclinical research in rodents has suggested that cold-induced activation of BAT leads to the clearance of circulating triglycerides (Bartelt et al., 2011; Berbee et al., 2015). Here, we tested the hypothesis that also in humans, cold exposure would lead to a reduction in post-prandial triglyceride levels. Therefore it was hypothesized that cold exposure would acutely lower triglycerides due to the increased usage of lipids during cold exposure in muscle and BAT. In addition, we wanted to test the hypothesis that cold exposure before a meal would deplete intracellular lipid stores in BAT, leading to an increased postprandial triglyceride uptake after cold exposure to replenish lipid stores in BAT. Therefore, we performed postprandial triglyceride kinetics during and upon cold exposure. However, contrary to our hypothesis and in contrast with findings in animals, we observed an increase in both postprandial triglycerides and FFA levels during cold exposure, which may partially be explained by an increase in lipolysis that occurs with acute cold exposure (Ouellet et al., 2012). Furthermore, pre-cooling also did not lower postprandial triglycerides or FFA levels. Together, these results are in contrast to the findings in preclinical studies and suggest that in humans acute cold exposure does not lead to enhanced lipid clearance.

We previously showed that cold acclimation improved substrate metabolism and insulin sensitivity in type 2 diabetes (Hanssen et al., 2015). Here, we found that postprandial glucose levels were lower with acute cold exposure and pre-cooling, which suggests that acute cold exposure could have a potential positive effect on glucose clearance and would be consistent with our previous finding of beneficial effects of cold exposure on insulin sensitivity (Hanssen et al., 2015). In the latter study, we showed that cold exposure can lead to an increase in skeletal muscle GLUT4 translocation. The improved glucose clearance upon cold exposure observed in the current study, could be due to enhanced GLUT4 translocation in muscle tissue.

Although brown adipose tissue is a potent tissue, in humans the amount of brown adipose tissue may not be enough to affect lipid consumption and therefore lower plasma levels, as was discussed previously (Fernandez-Verdejo et al., 2019). Skeletal muscle would be another tissue that could contribute to lipid consumption during cold exposure due to increased leak respiration (Bal et al., 2017; Blondin et al., 


\section{Chapter 3}

2017a). However, we did not observe any effects in maximal coupled or uncoupled respiration between the thermoneutral and cold conditions. Furthermore, we did not observe differences in leak respiration (state 4o) in skeletal muscle tissue, which indicates an absence of any increased proton leak during acute cold exposure. This absence has also been observed in another cold exposure study (Wijers et al., 2008).

Although acute cold exposure is sufficient to lower lipid levels in rodents (Bartelt et al., 2011), in humans the acute cold exposure without shivering did not lead to decreased lipid levels. Additionally, there is the option that longer-term cold acclimation could have a significant effect on triglyceride clearance. In cold-adapted winter swimmers, lower levels of markers of cardiovascular risk compared to non-adapted controls have been found (Kralova Lesna et al., 2015). Therefore, future studies might focus on longer periods of cold exposure or on the effects of shivering thermogenesis, in order to investigate the potential of cold as a treatment option to combat cardiovascular diseases. 
Cold exposure, triglycerides and mitochondrial function

\section{Supplementary data for chapter 3}

Table S1. Nutritional information of meal test shake

\begin{tabular}{lll}
\hline & Content & Energy \% \\
\hline Energy & $755 \mathrm{kcal}$ & 100 \\
Fat & $50.8 \mathrm{~g}$ & 60.6 \\
$\quad$ Saturated fat & $26.1 \mathrm{~g}$ & 31.1 \\
$\quad$ Unsaturated fat & $23.8 \mathrm{~g}$ & 28.4 \\
$\quad$ Cholesterol & $0.9 \mathrm{~g}$ & 1.1 \\
Carbohydrate & $62.3 \mathrm{~g}$ & 33.0 \\
Protein & $10.9 \mathrm{~g}$ & 5.8 \\
\hline
\end{tabular}




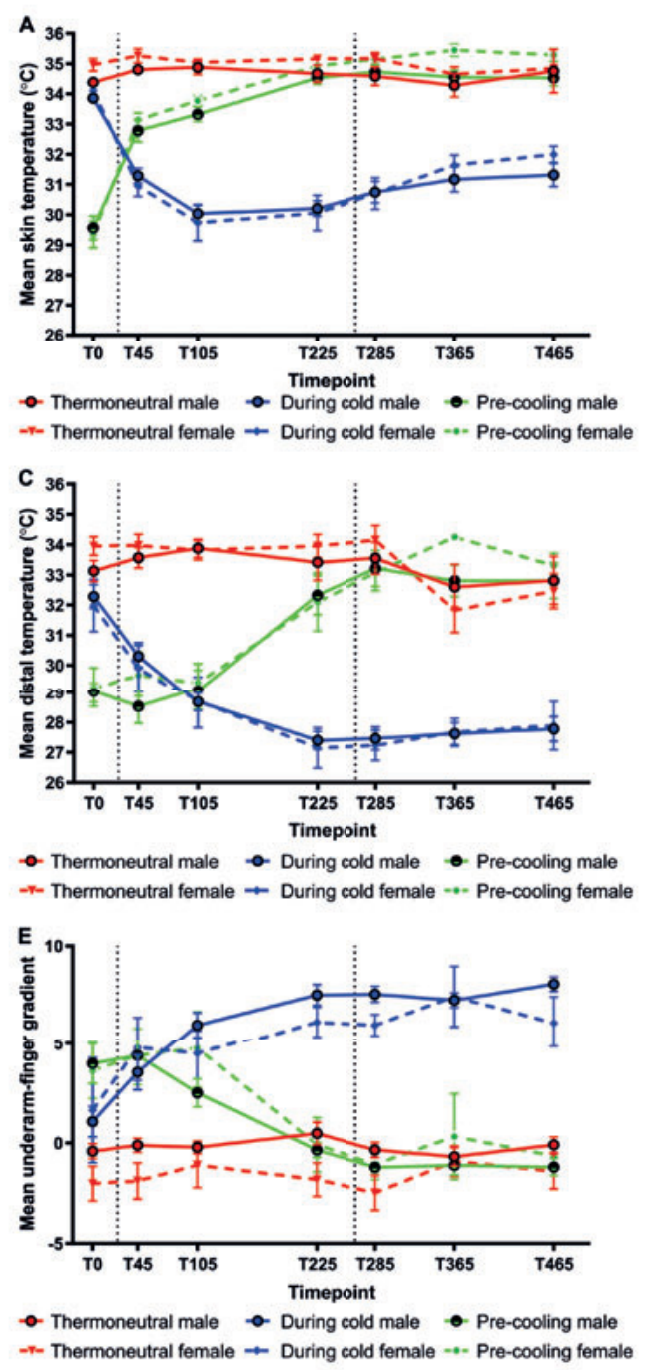

Figure S2. Skin temperatures during the meal tests for different genders. A) Mean skin temperature during the different meal tests. B) Mean proximal skin temperature. C) Mean distal skin temperature, D) Mean proximal-distal gradient and E) Mean underarm-finger gradient. The meal test at thermoneutrality is indicated as the red line, the meal test during cold exposure as the blue line, and the meal test after cold as the green line. Dashed vertical lines show the time of consumption of the 1 st shake at T0 and the 2nd shake at T240. Data is presented as mean \pm SEM ( $n=14 ; 9$ males, 5 females). 

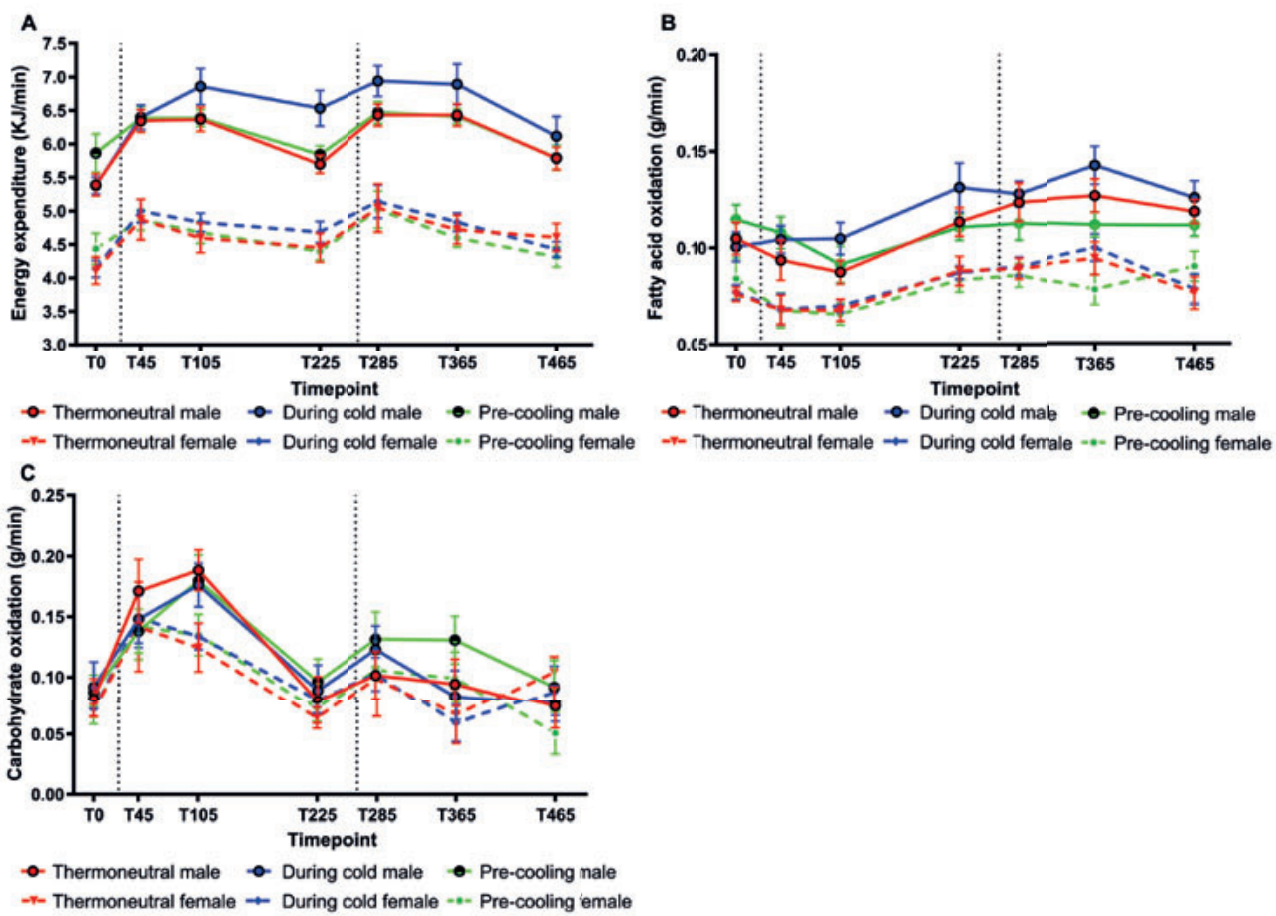

Figure S3. Energy expenditure and substrate oxidation during the meal tests for different genders. Energy expenditure $(\boldsymbol{A})$, fatty acid oxidation $(\boldsymbol{B})$ and carbohydrate oxidation $(\boldsymbol{C})$ during the meal tests. The meal test at thermoneutrality is indicated as the red line, the meal test during cold exposure as the blue line and the meal test after cold as the green line. Dashed vertical lines indicate the time of consumption of the 1 st shake at TO and the $2 n d$ shake at T240. Data is presented as mean \pm SEM ( $n=14 ; 9$ males, 5 females). 
Chapter 3

A

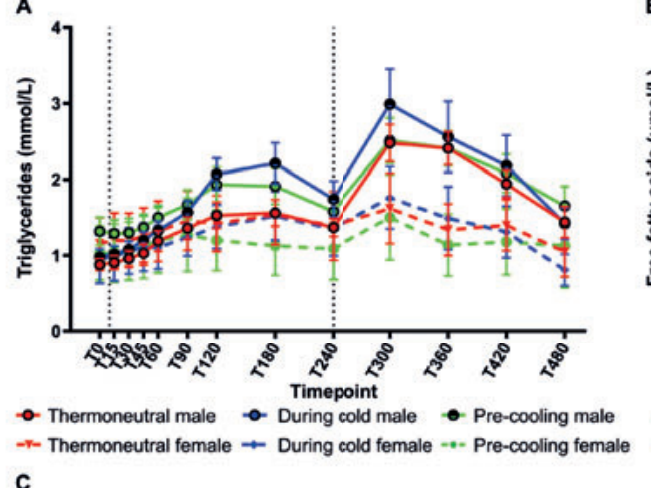

C
B

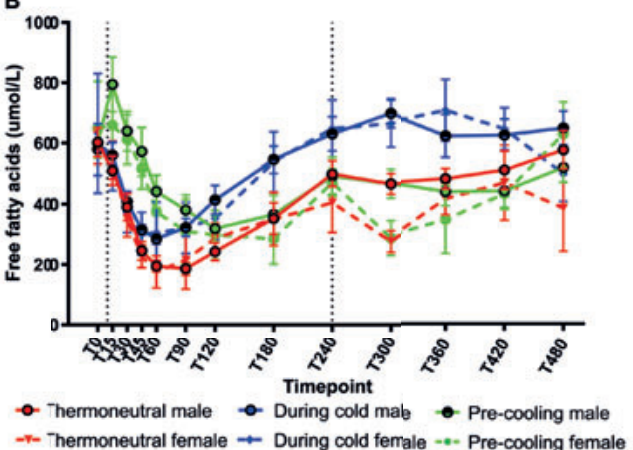

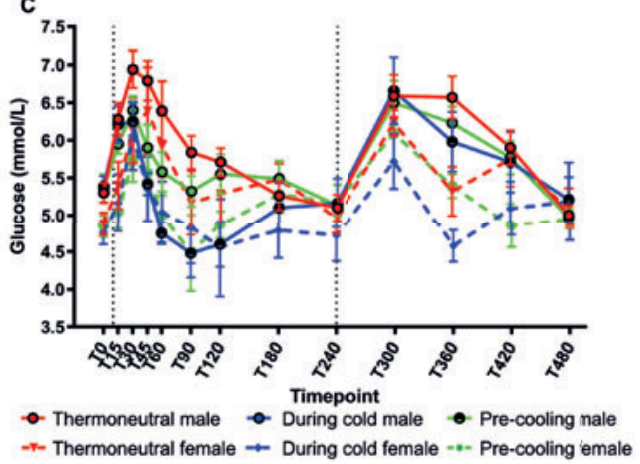

Figure S4. Plasma levels during the meal tests for different genders. Plasma levels of triglycerides (A), free fatty acids (B) and glucose (C) during the meal tests. The meal test at thermoneutrality is represented as the red line, the meal test during cold as the blue line and the meal test after cold as the green line. Dashed vertical lines indicate the time of consumption of the 1st shake at T0 and the 2nd shake at T240. Data is presented as mean \pm SEM ( $n=14 ; 9$ males, 5 females). 


\section{References}

Austin MA, McKnight B, Edwards KL, Bradley CM, McNeely MJ, Psaty BM, Brunzell JD \& Motulsky AG. (2000). Cardiovascular disease mortality in familial forms of hypertriglyceridemia: A 20-year prospective study. Circulation 101, 2777-2782.

Baba S, Jacene HA, Engles JM, Honda H \& Wahl RL. (2010). CT Hounsfield units of brown adipose tissue increase with activation: preclinical and clinical studies. J Nucl Med 51, 246-250.

Bal NC, Maurya SK, Pani S, Sethy C, Banerjee A, Das S, Patnaik S \& Kundu CN. (2017). Mild cold induced thermogenesis: are BAT and skeletal muscle synergistic partners? Biosci Rep 37.

Bartelt A, Bruns OT, Reimer R, Hohenberg H, Ittrich H, Peldschus K, Kaul MG, Tromsdorf UI, Weller H, Waurisch C, Eychmuller A, Gordts PL, Rinninger F, Bruegelmann K, Freund B, Nielsen P, Merkel M \& Heeren J. (2011). Brown adipose tissue activity controls triglyceride clearance. Nat Med 17, 200-205.

Baumgartner S, Mensink RP \& Plat J. (2016). Effects of a Plant Sterol or Stanol Enriched Mixed Meal on Postprandial Lipid Metabolism in Healthy Subjects. PLoS One 11, e0160396.

Berbee JF, Boon MR, Khedoe PP, Bartelt A, Schlein C, Worthmann A, Kooijman S, Hoeke G, Mol IM, John C, Jung C, Vazirpanah N, Brouwers LP, Gordts PL, Esko JD, Hiemstra PS, Havekes LM, Scheja L, Heeren J \& Rensen PC. (2015). Brown fat activation reduces hypercholesterolaemia and protects from atherosclerosis development. Nat Commun 6, 6356 .

Bergström J, Hermansen L, Hultman E \& Saltin B. (1967). Diet, muscle glycogen and physical performance. Acta Physiol Scand 71, 140-150.

Blondin DP, Daoud A, Taylor T, Tingelstad HC, Bezaire V, Richard D, Carpentier AC, Taylor AW, Harper ME, Aguer C \& Haman F. (2017a). Four-week cold acclimation in adult humans shifts uncoupling thermogenesis from skeletal muscles to brown adipose tissue. J Physiol 595, 2099-2113.

Blondin DP, Tingelstad HC, Noll C, Frisch F, Phoenix S, Guerin B, Turcotte EE, Richard D, Haman F \& Carpentier AC. (2017b). Dietary fatty acid metabolism of brown adipose tissue in cold-acclimated men. Nat Commun 8, 14146.

Boellaard R, O'Doherty MJ, Weber WA, Mottaghy FM, Lonsdale MN, Stroobants SG, Oyen WJ, Kotzerke J, Hoekstra OS, Pruim J, Marsden PK, Tatsch K, Hoekstra CJ, Visser EP, Arends B, Verzijlbergen FJ, Zijlstra JM, Comans EF, Lammertsma AA, Paans AM, Willemsen AT, Beyer T, Bockisch A, Schaefer-Prokop C, Delbeke D, Baum RP, Chiti A \& Krause BJ. (2010). FDG PET and PET/CT: EANM procedure guidelines for tumour PET imaging: version 1.0. Eur J Nucl Med Mol Imaging 37, 181-200.

Cannon B \& Nedergaard J. (2004). Brown adipose tissue: function and physiological significance. Physiol Rev 84, 277-359.

Chondronikola M, Volpi E, Borsheim E, Porter C, Saraf MK, Annamalai P, Yfanti C, Chao T, Wong D, Shinoda K, Labbe SM, Hurren NM, Cesani F, Kajimura S \& Sidossis LS. (2016). Brown Adipose Tissue Activation Is Linked to Distinct Systemic Effects on Lipid Metabolism in Humans. Cell Metab 23, 1200-1206.

Dauncey MJ. (1980). Metabolic effects of altering the $24 \mathrm{~h}$ energy intake in man, using direct and indirect calorimetry. Br J Nutr 43, 257-269.

Dong M, Yang X, Lim S, Cao Z, Honek J, Lu H, Zhang C, Seki T, Hosaka K, Wahlberg E, Yang 
J, Zhang L, Lanne T, Sun B, Li X, Liu Y, Zhang Y \& Cao Y. (2013). Cold exposure promotes atherosclerotic plaque growth and instability via UCP1-dependent lipolysis. Cell Metab 18, 118-129.

Fernandez-Verdejo R, Marlatt KL, Ravussin E \& Galgani JE. (2019). Contribution of brown adipose tissue to human energy metabolism. Mol Aspects Med 68, 82-89.

Hanssen MJ, Hoeks J, Brans B, van der Lans AA, Schaart G, van den Driessche JJ, Jorgensen JA, Boekschoten MV, Hesselink MK, Havekes B, Kersten S, Mottaghy FM, van Marken Lichtenbelt WD \& Schrauwen P. (2015). Short-term cold acclimation improves insulin sensitivity in patients with type 2 diabetes mellitus. Nat Med 21, 863-865.

Hanssen MJ, van der Lans AA, Brans B, Hoeks J, Jardon KM, Schaart G, Mottaghy FM, Schrauwen P \& van Marken Lichtenbelt WD. (2016). Short-term Cold Acclimation Recruits Brown Adipose Tissue in Obese Humans. Diabetes 65, 1179-1189.

Hoeks J, van Herpen NA, Mensink M, Moonen-Kornips E, van Beurden D, Hesselink MK \& Schrauwen P. (2010). Prolonged fasting identifies skeletal muscle mitochondrial dysfunction as consequence rather than cause of human insulin resistance. Diabetes 59, 2117-2125.

Hokanson JE \& Austin MA. (1996). Plasma triglyceride level is a risk factor for cardiovascular disease independent of high-density lipoprotein cholesterol level: a meta-analysis of population-based prospective studies. J Cardiovasc Risk 3, 213-219.

Khedoe PP, Hoeke G, Kooijman S, Dijk W, Buijs JT, Kersten S, Havekes LM, Hiemstra PS, Berbée JF, Boon MR \& Rensen PC. (2015). Brown adipose tissue takes up plasma triglycerides mostly after lipolysis. J Lipid Res 56, 51-59.

Kralova Lesna I, Rychlikova J, Vavrova L \& Vybiral S. (2015). Could human cold adaptation decrease the risk of cardiovascular disease? J Therm Biol 52, 192-198.

Muzik O, Mangner TJ, Leonard WR, Kumar A, Janisse J \& Granneman JG. (2013). 15O PET measurement of blood flow and oxygen consumption in cold-activated human brown fat. J Nucl Med 54, 523-531.

Nedergaard J, Golozoubova V, Matthias A, Asadi A, Jacobsson A \& Cannon B. (2001). UCP1: the only protein able to mediate adaptive non-shivering thermogenesis and metabolic inefficiency. Biochim Biophys Acta 1504, 82-106.

Nordestgaard BG, Benn M, Schnohr P \& Tybjaerg-Hansen A. (2007). Nonfasting triglycerides and risk of myocardial infarction, ischemic heart disease, and death in men and women. Jama 298, 299-308.

Nordestgaard BG \& Varbo A. (2014). Triglycerides and cardiovascular disease. Lancet 384, 626-635.

Ouellet V, Labbe SM, Blondin DP, Phoenix S, Guerin B, Haman F, Turcotte EE, Richard D \& Carpentier AC. (2012). Brown adipose tissue oxidative metabolism contributes to energy expenditure during acute cold exposure in humans. J Clin Invest 122, 545-552.

Péronnet F \& Massicotte D. (1991). Table of nonprotein respiratory quotient: an update. Can J Sport Sci 16, 23-29.

Vallerand AL \& Jacobs I. (1989). Rates of energy substrates utilization during human cold exposure. Eur J Appl Physiol Occup Physiol 58, 873-878.

van der Lans AA, Hoeks J, Brans B, Vijgen GH, Visser MG, Vosselman MJ, Hansen J, Jorgensen JA, Wu J, Mottaghy FM, Schrauwen P \& van Marken Lichtenbelt WD. (2013). Cold acclimation recruits human brown fat and increases nonshivering thermogenesis. J Clin Invest 123, 3395-3403. 
van Marken Lichtenbelt WD, Daanen HA, Wouters L, Fronczek R, Raymann RJ, Severens NM \& Van Someren EJ. (2006). Evaluation of wireless determination of skin temperature using iButtons. Physiol Behav 88, 489-497.

van Marken Lichtenbelt WD \& Schrauwen P. (2011). Implications of nonshivering thermogenesis for energy balance regulation in humans. Am J Physiol Regul Integr Comp Physiol 301, R285-296.

van Marken Lichtenbelt WD, Vanhommerig JW, Smulders NM, Drossaerts JM, Kemerink GJ, Bouvy ND, Schrauwen P \& Teule GJ. (2009). Cold-activated brown adipose tissue in healthy men. N Engl J Med 360, 1500-1508.

Vijgen GH, Bouvy ND, Teule GJ, Brans B, Schrauwen P \& van Marken Lichtenbelt WD. (2011). Brown adipose tissue in morbidly obese subjects. PLoS One 6, e17247.

Virtanen KA, Lidell ME, Orava J, Heglind M, Westergren R, Niemi T, Taittonen M, Laine J, Savisto NJ, Enerbäck S \& Nuutila P. (2009). Functional brown adipose tissue in healthy adults. N Engl J Med 360, 1518-1525.

Vosselman MJ, Brans B, van der Lans AA, Wierts R, van Baak MA, Mottaghy FM, Schrauwen P \& van Marken Lichtenbelt WD. (2013). Brown adipose tissue activity after a highcalorie meal in humans. Am J Clin Nutr 98, 57-64.

Vosselman MJ, Hoeks J, Brans B, Pallubinsky H, Nascimento EB, van der Lans AA, Broeders EP, Mottaghy FM, Schrauwen P \& van Marken Lichtenbelt WD. (2015). Low brown adipose tissue activity in endurance-trained compared with lean sedentary men. Int J Obes (Lond) 39, 1696-1702.

Weir G, Ramage LE, Akyol M, Rhodes JK, Kyle CJ, Fletcher AM, Craven TH, Wakelin SJ, Drake AJ, Gregoriades ML, Ashton C, Weir N, van Beek EJR, Karpe F, Walker BR \& Stimson RH. (2018). Substantial Metabolic Activity of Human Brown Adipose Tissue during Warm Conditions and Cold-Induced Lipolysis of Local Triglycerides. Cell Metab 27, 1348-1355 e1344.

Weir JB. (1949). New methods for calculating metabolic rate with special reference to protein metabolism. J Physiol 109, 1-9.

WHO (2018). Global Health Estimates 2016: Deaths by Cause, Age, Sex, by Country and by Region, 2000-2016. World Health Organization, Geneva.

Wijers SL, Schrauwen P, Saris WH \& van Marken Lichtenbelt WD. (2008). Human skeletal muscle mitochondrial uncoupling is associated with cold induced adaptive thermogenesis. PLoS One 3, e1777.

Wijers SL, Schrauwen P, van Baak MA, Saris WH \& van Marken Lichtenbelt WD. (2011). Beta-adrenergic receptor blockade does not inhibit cold-induced thermogenesis in humans: possible involvement of brown adipose tissue. J Clin Endocrinol Metab 96, E598-605. 


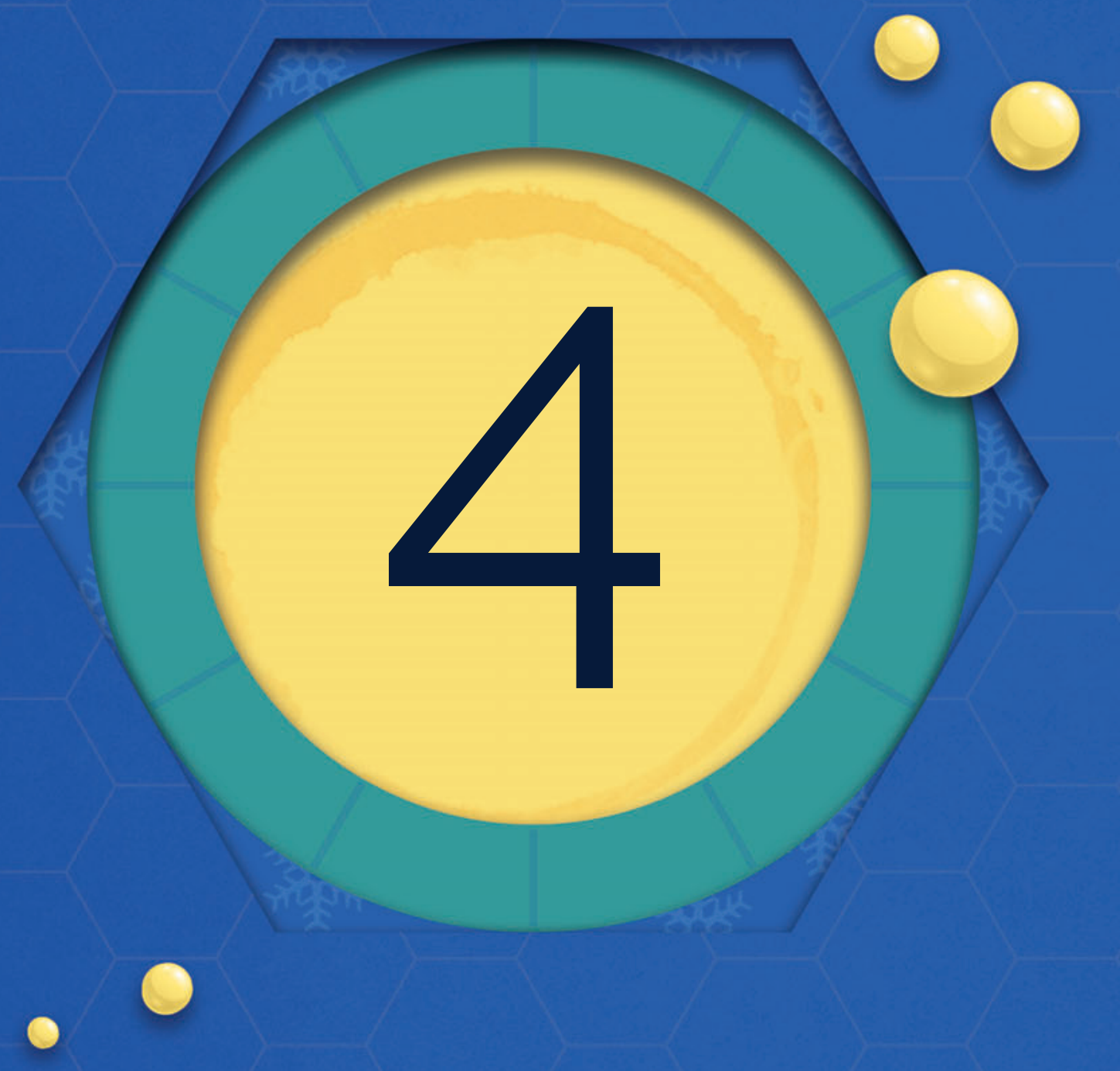




\section{Circadian misalignment disturbs the skeletal muscle lipidome in healthy young men}

Jan-Frieder Harmsen, Nynke van Polanen, Michel van Weeghel, Jakob Wefers, Joris Hoeks, Frédéric M. Vaz, Mia L. Pras-Raves, Antoine H. C. van Kampen, Gert Schaart, Dirk van Moorsel, Jan Hansen, Matthijs K. C. Hesselink, Riekelt H. Houtkooper and Patrick Schrauwen 


\section{Chapter 4}

\section{Abstract}

Circadian misalignment, as seen in shift work, is associated with an increased risk to develop type 2 diabetes. In an experimental setting, we recently showed that a rapid day-night shift for three consecutive nights leads to misalignment of the core molecular clock, induction of the PPAR pathway, and insulin resistance in skeletal muscle of young, healthy men. Here, we investigated if circadian misalignment affects the skeletal muscle lipidome and intramyocellular lipid droplet characteristics explaining the misalignment-induced insulin resistance. Fourteen healthy men underwent one aligned and one circadian misalignment period, both consisting of $\sim 3.5$ days. In the misaligned condition, day and night were rapidly shifted by 12 hours leading to opposite eating, sleep and activity times compared with the aligned condition. For each condition, two muscle biopsies were taken from the m. vastus lateralis in the morning and evening and subjected to semi-targeted lipidomics and confocal microscopy analysis. We found that only $2 \%$ of detected lipids were different between morning and evening in the aligned condition, whereas $12 \%$ displayed a morning-evening difference upon misalignment. Triacylglycerols, in particular species of a carbon length $\geq 55$, were the most abundant lipid species changed upon misalignment. Cardiolipins were decreased upon misalignment, whereas phosphatidylcholines consistently followed the same morning-evening pattern, suggesting regulation by the circadian clock. Cholesteryl esters adjusted to the shifted behaviour. Lipid droplet characteristics remained unaltered upon misalignment. Together, these findings show that simulated shift work disturbs the skeletal muscle lipidome, which may contribute to misalignment-induced insulin resistance. 


\section{Introduction}

Our 24-hour culture, characterized by working and eating late, reduced sleep quantity and quality, and excessive light exposure at night is more and more recognized for its negative impact on metabolic health (Koshy et al., 2019) and the development of type 2 diabetes mellitus (T2DM) (Sulli et al., 2018; Gutierrez-Monreal et al., 2020). In humans, skeletal muscle insulin resistance is an early hallmark in the development of T2DM (DeFronzo \& Tripathy, 2009) and we recently demonstrated that circadian misalignment introduced by a rapid day-night shift for as short as three consecutive nights already resulted in a $23 \%$ reduction of skeletal muscle insulin sensitivity in comparison with a control condition of similar duration (Wefers et al., 2018). The impaired insulin sensitivity was accompanied by changes in muscle gene expression related to peroxisome proliferator-activated receptor (PPAR) signalling and fat metabolism as well as by elevated overnight fasted plasma free

fatty acid levels (Wefers et al., 2018), suggesting that a disturbed skeletal muscle lipid metabolism is involved in the development of muscle insulin resistance upon circadian misalignment. Indeed, it has been shown on numerous occasions that accumulation of lipids and/or certain lipid species within skeletal muscle cells can contribute to the development of muscle insulin resistance (Amati et al., 2011; Nielsen et al., 2017; Daemen et al., 2018). Interestingly, we have recently shown in young healthy volunteers that the skeletal muscle lipidome displays 24-hour rhythmicity (Held et al., 2020); whether this rhythmicity is driven by the circadian clock or due to feeding and fasting schedules could not be deduced. Nevertheless, together with the previously described diurnal fluctuations in insulin sensitivity, with greater sensitivity in the morning hours (Van Cauter et al., 1991; Saad et al., 2012), these findings suggest that 24-hour rhythms in the muscle lipidome and insulin sensitivity may be linked and that circadian misalignment could disturb the lipidome, which in turn may contribute to the development of insulin resistance.

To test this hypothesis, we here investigated the effect of circadian misalignment on the skeletal muscle lipidome as well as on intramyocellular lipid droplet characteristics, using semi-quantitative ultrahigh-performance liquid chromatography-high 


\section{Chapter 4}

resolution mass spectrometry (UPLC/HRMS)- based lipidomics and high resolution confocal microscopy, respectively. To this end, we analysed muscle biopsies taken from our recent study (Wefers et al., 2018), in which participants spent 3-4 days under controlled laboratory conditions adhering either to a control or a circadian misalignment protocol reinforced by scheduled meals and physical activity during the respective day-time period and sleep opportunity during the respective nighttime period. In both conditions, two muscle biopsies were taken, one in the respective behavioural fasted morning ( $8 \mathrm{AM}$ or $8 \mathrm{PM}$ ) and one in the behavioural fed evening (8 PM or $8 \mathrm{AM}$ ), enabling us to investigate muscle lipid metabolism under realistic day-night shift conditions. In addition, comparing morning-evening differences in the aligned and misaligned condition can also reveal information on the respective influence of the circadian versus behavioural clock on the muscle lipidome.

\section{Material and Methods}

\section{Participants}

Fourteen young healthy lean male individuals [age: $22.4 \pm 2.8$ years; body mass index (BMI): $22.3 \pm 2.1 \mathrm{~kg} / \mathrm{m}^{2}$; mean $\pm \mathrm{SD}$ ] participated in this study. Participants were nonsmokers, had no active diseases, used no medication, and did not engage in exercise for more than 3 hours per week, as verified by questionnaires. Moreover, participants had regular bedtimes (11 PM \pm 2 hours), regular sleep duration of 7-9 hours, did not perform shift work or travel across more than one time zone in the 3 months preceding the study, and were not definite morning larks or night owls, assessed with a Morningness-Eveningness Questionnaire Self-Assessment (MEQ-SA: $54 \pm 7$, mean $\pm S D$ ). The study was conducted according to the principles of the declaration of Helsinki and approved by the Ethics Committee of the Maastricht University Medical Centre. All participants provided written informed consent. The study was registered at clinicaltrials.gov with identifier NCT02580513. 


\section{Study design}

Study procedures have been described in detail before (Wefers et al., 2018). Briefly, each participant underwent one control period and one circadian misalignment period in a randomized cross-over design with a wash-out period of 4-10 weeks. In the aligned and misaligned condition, participants stayed in a respiration chamber for 3 and 3.5 days, respectively. Lighting in the chamber was set to 4 lux when participants were awake and switched off during sleeping opportunities. Participants had no information on time during their entire stay in the chamber. In the control condition, participants adhered to a normal diurnal lifestyle, including scheduled sleep from 11 PM to 7 AM and meals provided at 8 AM, 12:30 PM, 3 PM and 8 PM. In the circadian misalignment condition, the behavioural cycle was shifted by 12 hours on the second day spent in the chamber. At 3 PM, participants had to switch off the lights and try to sleep lying down until 7 PM. Subsequently, participants behaved as if it was a new morning and adhered to the same series of scheduled meals followed by sleeping from 11 AM until 7 PM. Light physical activity (in the form of stepping) was performed 1 hour after each meal in both conditions to prevent sedentary behaviour. The study design is summarized in Figure 1.

\section{Study meals}

All study meals were standardized and in line with the Dutch and US dietary guidelines. Caloric requirements for the study days were calculated by multiplying sleeping metabolic rate as determined during the first night of each condition with an activity factor of 1.5. Daily caloric intake was distributed over three meals and a snack. Breakfast accounted for $\sim 20 \%$, lunch for $\sim 25 \%$, a snack for $\sim 10 \%$, and dinner for $\sim 45 \%$ of energy. Overall daily macronutrient distribution was $\sim 52 \%$ as carbohydrates, $\sim 31 \%$ as fat ( $\sim 9 \%$ saturated), and $\sim 14 \%$ as protein. Breakfast and lunch mostly consisted of bread and cold cuts, whereas dinner was a hot meal, contributing to a relatively higher fat content for dinner, as is typical in the Netherlands 


\section{Aligned condition:}

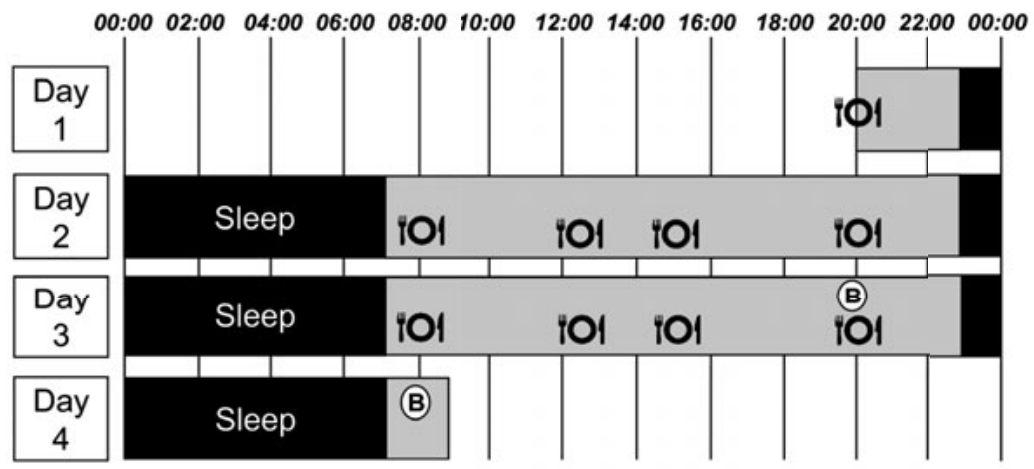

Misaligned condition:

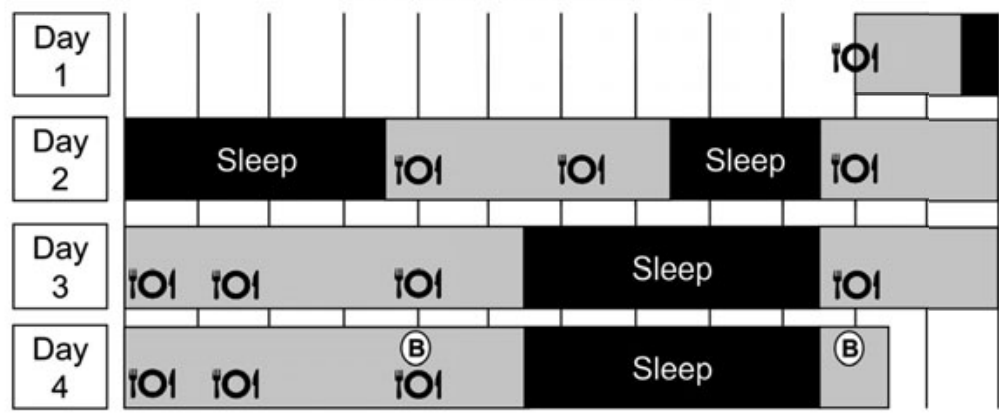

$\square$ = Dim light (4 lux) $\square$ = Dark (lights out) IOI = Meal (breakfast, lunch,

(B) $=$ Muscle biopsy snack and dinner)

Figure 1. Study design.

\section{Skeletal muscle biopsies}

For each condition, two skeletal muscle biopsies were taken from the m. vastus lateralis under local anaesthesia (1\% lidocaine, without epinephrine) at $8 \mathrm{AM}$ and 8 PM corresponding to the behavioural morning and evening in the aligned condition and behavioural evening and morning in the misaligned condition, respectively. Consecutive biopsies were obtained by moving from distal to proximal. Whether the left or right leg was used in the first condition for the first biopsy was randomized, and the subsequent biopsy was performed on the other leg, alternating thereafter between both legs. Muscle tissue was immediately frozen in melting isopentane and stored at $-80^{\circ} \mathrm{C}$ for further analysis. 


\section{Immunohistochemistry}

For histochemical analysis, the two muscle biopsies taken in the behavioural morning in the fasted state (aligned: $8 \mathrm{AM}$, misaligned: $8 \mathrm{PM}$ ) were subjected to confocal microscopy analysis to assess lipid droplet (LD) morphology $(n=13)$. For one participant, muscle tissue could not be used for LD analysis due to bad tissue morphology. Muscle samples were cut in 5- $\mu \mathrm{m}$ thick sections and mounted on glass slides. A section from the aligned and misaligned condition from one participant was mounted on the same glass slide to minimize staining intensity variability between conditions. Cryosections were air-dried at room temperature (RT) for 30 minutes and permeabilized for 5 minutes with 0.25\% TX-100 (648466, Merck, Darmstadt, Germany). Primary antibodies against Laminin (L9393; Sigma-Aldrich; 1:25) and myosin heavy chain type I (A4.840; Developmental Studies Hybridoma Bank; 1:25) were applied and sections were incubated for 45-60 minutes in a humidified chamber at RT. Subsequently, sections were incubated for 90 minutes with the appropriate secondary antibodies conjugated with AlexaFluor 405 and AlexaFluor 555 at $37^{\circ} \mathrm{C}$. LDs were visualized with Bodipy 493/503 (D3922, Thermo Fisher Scientific; 1:100). After washing, sections were mounted with Mowiol and covered with \#1 coverslips and stored in the dark at RT until imaging.

\section{Visualization and quantification}

Muscle tissues were visualized and quantified as described previously (Daemen et al., 2018). In short, cross-sections of $\sim 20$ muscle fibres with previously determined type I/type II ratio were imaged under a Leica TCS SP8 confocal microscope using a $63 x 1.4$ N.A. oil immersion objective. Deconvolution was performed with Huygens Professional software (Scientific Volume Imaging B.V., Hilversum, the Netherlands), image quantification with Image J version 1.52p (NIH, Bethesda, USA) and further analysis with MATLAB R2013a (The Mathworks, Inc., Natick, Massachusetts, USA). Intramyocellular lipid content was calculated as the percentage of the total lipid area to the total cell area in type I muscle fibres, type II muscle fibres and in both muscle fibre types combined. LD number was total LD count corrected for cell area and 


\section{Chapter 4}

LD size was measured by total lipid area divided by the total LD count. Note that lipid content is the product of lipid droplet size and number. LD distribution, that is distinction between intermyofibrillar (IMF) LDs and subsarcolemmal (SS) LDs, was determined as described previously (Daemen et al., 2018).

\section{Lipidomics analysis}

Lipid extraction and lipidomics analysis were performed as described previously (Herzog et al., 2016; Held et al., 2020). From the entire 1478 lipids detected in all four biopsies, 130 lipids with a relative abundance of less than 0.05 and 177 lipids with pronounced variation in the quality control batch (coefficient of variation $>30 \%$ ) were excluded from further analyses. Thus, 1171 lipids were subjected to further analyses. Subclustering into different lipid classes was conducted as described previously (Held et al., 2020).

\section{Statistical analysis}

Data are presented as mean \pm SEM (standard error of the mean). The effect of circadian misalignment on LD characteristics was assessed by paired samples t-tests as only the behavioural morning of each condition (Aligned: 8 AM, Misaligned: 8 $\mathrm{PM}$ ) was subjected to this analysis. To test for changes in the muscle lipidome, all four time points were included (Aligned: $8 \mathrm{AM}$ and $8 \mathrm{PM}$, Misaligned: $8 \mathrm{AM}$ and $8 \mathrm{PM})$. Therefore, two-way repeated measures ANOVAs were calculated to reveal time (8 AM vs 8 PM), condition (aligned vs misaligned) or interaction effects. To identify lipid species with a morning vs evening difference in both conditions, paired samples t-tests were conducted for each lipid species within each condition. To correlate altered lipid classes / species to muscle insulin sensitivity, Pearson correlation coefficients were calculated. The level of significance was set to $<0.05$ for all analyses. Statistical analyses were performed using an in-house developed metabolomics pipeline (Herzog et al., 2016) written in the R programming language (http://www.r-project.org) and further processed with the GraphPad Prism 8 software package (GraphPad Software, San Diego, CA, USA). 


\section{Results}

\section{Lipid droplet morphology did not change upon misalignment}

We have previously shown that our misalignment protocol of 3.5 days resulted in compromised insulin sensitivity in the peripheral tissue, especially skeletal muscle (Wefers et al., 2018). Insulin sensitivity was determined by a hyperinsulinemic euglycemic clamp after an "overnight" fast, either at 8 AM (aligned) or 8 PM (misaligned) following the respective muscle biopsy. As intramyocellular lipid content is related to insulin sensitivity in non-athletes (Gemmink et al., 2017), we first investigated in the present study if the reduction in insulin sensitivity upon circadian misalignment is accompanied by altered lipid droplet morphology. No difference in total lipid content (mixed fibre type) was observed between the aligned and misaligned condition $(0.330 \pm 0.052 \%$ vs $0.324 \pm 0.042 \%$, respectively, $p=0.886$,

Figure 2A). As expected, type I muscle fibres had higher lipid content compared with type II muscle fibres $(\mathrm{p}<0.01)$, but there were no differences in total lipid content between the aligned and misaligned condition in either fibre type.

Similar amounts of lipids can be stored in more but smaller, or fewer but larger LDs and the latter LD characteristics have been associated with insulin resistance (Covington et al., 2017; Nielsen et al., 2017; Daemen et al., 2018). Therefore, we next examined if LD size and number were affected by circadian misalignment. However, LD number was not significantly different between the aligned and misaligned condition in both fibre types $\left(0.015 \pm 0.002 \# / \mu \mathrm{m}^{2} \mathrm{vs} 0.014 \pm 0.002 \# / \mu \mathrm{m}^{2}\right.$, respectively, $\mathrm{p}=0.772$, Figure 2B), neither in type I nor type II muscle fibres when analysed separately. Similarly, LD size was not significantly different between the aligned and misaligned conditions $\left(0.219 \pm 0.005 \mu \mathrm{m}^{2}\right.$ vs $0.223 \pm 0.006 \mu \mathrm{m}^{2}, \mathrm{p}=0.374$, both muscle fibres, Figure 2C), irrespective of muscle fibre type. 


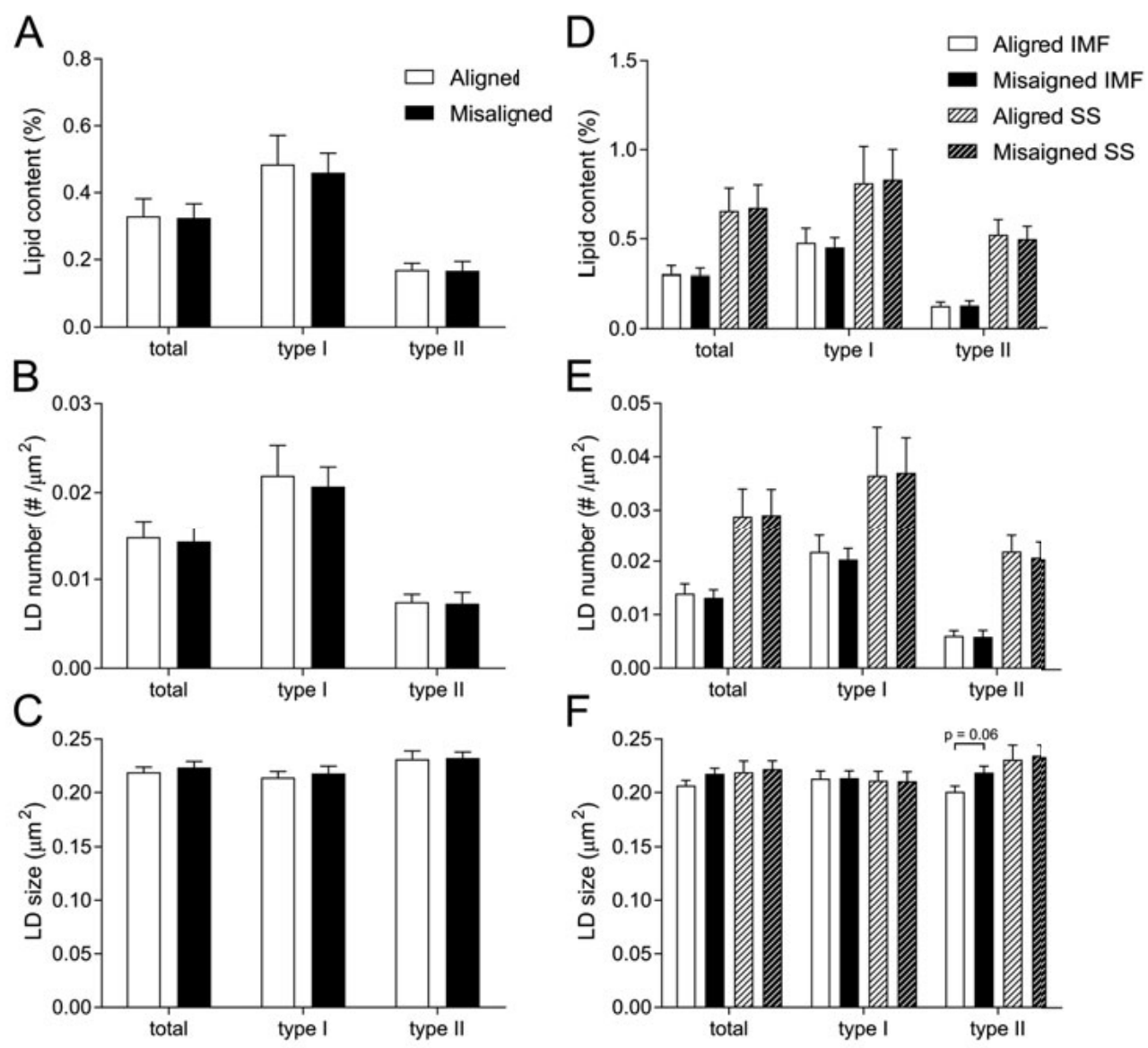

Figure 2. Lipid droplet (LD) morphology did not change upon circadian misalignment. Lipid content $(\boldsymbol{A}), L D$ number $(\boldsymbol{B})$ and $L D$ size $(\boldsymbol{C})$ were not significantly different between the aligned (8 AM) and misaligned (8 PM) condition. Distribution of LDs within the muscle cell, either intermyofibrillar (IMF) or subsarcolemmal (SS) did also not reveal differences (D-F). Intermyofibrillar LD size in type II fibres $(\boldsymbol{F})$ tended to be increased upon misalignment ( $p=0.06$; paired t-test). Data are displayed as mean $\pm S E M(n=13)$. 


\section{Misalignment did not affect intramyocellular lipid droplet Iocalization}

LDs within the muscle fibres can be distributed among the contractile elements or near the sarcolemma. Using confocal microscopy, we can distinguish between intermyofibrillar (IMF) LDs and subsarcolemmal (SS) LDs (Daemen et al., 2018). We found no differences in total lipid content (both muscle fibres) in the separate IMF and SS regions between the aligned vs misaligned condition (IMF: $0.304 \pm 0.047 \%$ vs $0.297 \pm 0.040 \%, p=0.872$; SS: $0.656 \pm 0.128 \%$ vs $0.675 \pm 0.129 \%, p=0.836$, Figure $2 \mathrm{D})$, nor in type I or type II muscle fibres separately.

Similar findings were obtained for LD number, as no differences in both muscle fibres between the aligned vs misaligned condition were observed (IMF: $0.014 \pm 0.002$ \#/ $\mu \mathrm{m}^{2}$ vs $0.013 \pm 0.001 \# / \mu \mathrm{m}^{2}, \mathrm{p}=0.637$; SS: $0.029 \pm 0.005 \# / \mu \mathrm{m}^{2}$ vs $0.029 \pm 0.005 \# / \mu \mathrm{m}^{2}$, $\mathrm{p}=0.933$, Figure 2E), nor for type I or type II muscle fibres. For LD size, no differences

were found in both muscle fibres and type I muscle fibres. LD size in type II muscle fibres, however, tended to increase in the IMF region, but did not reach statistical significance $\left(0.201 \pm 0.005 \mu \mathrm{m}^{2}\right.$ vs $0.219 \pm 0.006 \mu \mathrm{m}^{2} ; \mathrm{p}=0.060$, Figure $\left.2 \mathrm{~F}\right)$.

\section{Muscle lipidome displays greater morning-evening differences after misalignment}

Confocal microscopy provides interesting information on LD morphology, yet does not reveal data on the composition of the intramyocellular lipids. Therefore, we next performed a semi-quantitative lipidomics analysis to investigate if skeletal muscle lipid composition is altered upon circadian misalignment. For this purpose, we included all four time points (8 AM and 8 PM for both aligned and misaligned) in the analysis, enabling us to study if morning vs evening differences of individual muscle lipids were affected by misalignment. In total, we detected 1171 lipid species representing 23 lipid classes (Table 1) that were present in all participants. In the aligned condition, only 19 out of 1171 lipids (2\%) were significantly different between morning and evening (Figure 3A), whereas 101 (12\%) lipid species were different between morning and evening in the misaligned condition, i.e. respective lipid 


\section{Chapter 4}

species were predominantly higher in the behavioural morning after the overnight fast upon misalignment (Figure 3B). Out of the lipids demonstrating a significant morning vs evening difference, only two lipid species (TG(O-46:11) and DG(O42:5)) were shared in both conditions (Figure 3C). We next focused on the 101 lipids in which a morning-evening difference was introduced upon misalignment and summarized these lipids into clusters according to their chemical properties. Out of the 101 lipids, 54 lipids were triradylglycerols - an umbrella term that encompasses triacylglycerols (TG) and alkyldiacylglycerols (TG[O]) (13\% of all 404 detected lipids from this particular lipid cluster; $40 \mathrm{TG}$ and $14 \mathrm{TG}[\mathrm{O}]), 24$ were glycerophospholipids (5\%), 19 were sphingolipids (14\%), 3 were diradylglycerols (2\%, DG and DG[O]) and 1 was a cholesteryl ester (13\%) (Figure 3D).

Table 1. Overview of lipid clusters that are divided into separate lipid classes according to their chemical properties.

\begin{tabular}{|l|l|l|}
\hline Lipid cluster & Abbreviation & Common name \\
\hline Glycerophospho- & BMP & Bis(monoacylglycero)phosphate \\
& CL, MLCL & Cardiolipin, Monolysocardiolipin \\
& LPC & Lysophosphatidylcholine \\
& LPC[O] & Alkyl/alkenyllysophosphatidylcholine \\
& LPE & Lysophosphatidylethanolamine \\
& LPG & Alkyl/alkenyllysophosphatidylethanolamine \\
& PA & Lysophosphatidylglycerol \\
& PC & Phosphatidic acid \\
& PC[O] & Phosphatidylcholine \\
& PE & Alkyl/alkenylphosphatidylcholine \\
& PE[O] & Phosphatidylethanolamine \\
& PI & Alkyl/alkenylphosphatidylethanolamine \\
& PS & Phosphatidylinositol \\
& DG & Phosphatidylserine \\
\hline Diradylglycerol & DG[O] & Alkyl/alkenylacylglycerol \\
\hline lipids & TG & Triacylglycerol \\
\hline Triradylglycerol & TG[O] & Alkyl/alkenyldiacylglycerol \\
\hline lipids & Cer[d] & Ceramide \\
\hline Sphingolipids & HexCer[d] & Hexosylceramide \\
& SM & Sphingomyelin/Ceramide phosphocholine \\
& SPH & Sphingosine/Sphinganine \\
\hline Sterol lipids & CE & Cholesteryl ester \\
\hline
\end{tabular}




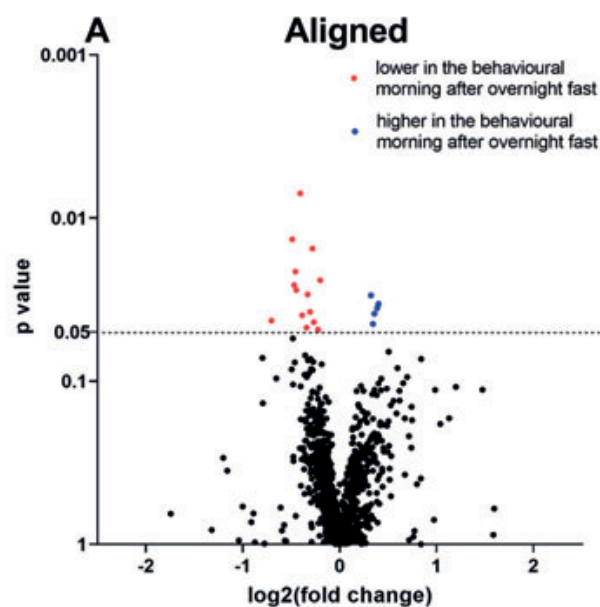

B Misaligned

\section{Morning vs. evening difference:}

D Lipids that changed within Misaligned:

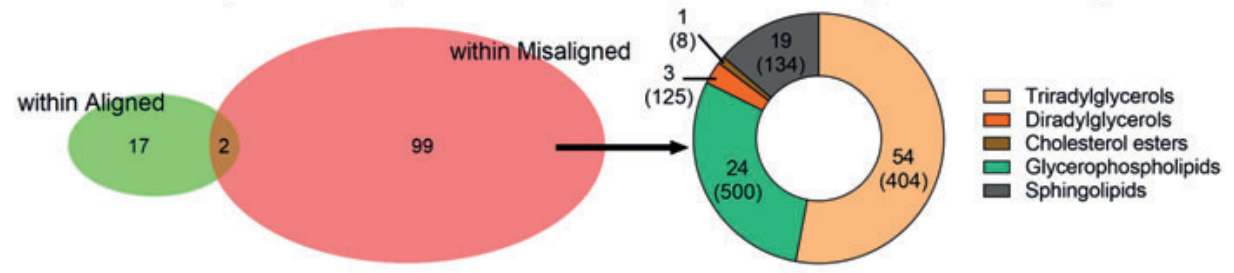

Figure 3. Volcano plots for the comparison ( $8 A M$ vs 8 PM) within the aligned $(A)$ and misaligned condition (B) for the entire muscle lipidome. A-B) A negative log2 fold change indicates that respective lipids were lower (red dots) in the behavioural morning after an overnight fast compared with the behavioural evening, and a positive log2 fold change indicates higher levels (blue dots). Note that only 19 out of 1171 lipids (2\%) were significantly different between morning and evening in the aligned condition, whereas 101 out of 1171 lipids (12\%) were different between morning and evening in the misaligned condition. A list with the names of the altered lipid species can be found in supplemental Tables S1 and S2. C) Out of all the lipids showing a morning vs evening difference, only two lipids were shared in both conditions. D) Classification of the 101 lipids significantly different between morning and evening within the misaligned condition according to the five main lipid clusters. Values in parentheses represent the overall detected absolute number of lipids in this cluster from the entire lipidome pool of 1171 lipids. Note that $\sim 13 \%$ of all triradylglycerols (triacylglycerols and alkyldiacylglycerols) were different between morning and evening in the misaligned condition. 


\section{Circadian misalignment alters triacylglycerol composition}

As triradylglycerols were the major lipid cluster in which morning-evening differences appeared after circadian misalignment, we next looked for patterns related to carbon chain length and saturation level within triradylglycerol species. Noticeably, the lipidomics platform we used does not provide the exact fatty acid composition of the lipids but only presents the total carbon chain length and the total number of double bonds (both of the radyl groups in the side-chains). Nonetheless, the morningevening differences in triradylglycerols induced by circadian misalignment showed a clear pattern with respect to chain length but not to saturation level. Indeed, in the misaligned condition, we observed a significant increase of longer triradylglycerol species ( $\geq 55$ carbons), which were higher in the behavioural morning (8 PM) as compared with the behavioural evening ( $8 \mathrm{AM})$. The different pattern between aligned and misaligned within the main subcluster of triradylglycerols, triacylglycerols (TG), is illustrated in Figure 4.

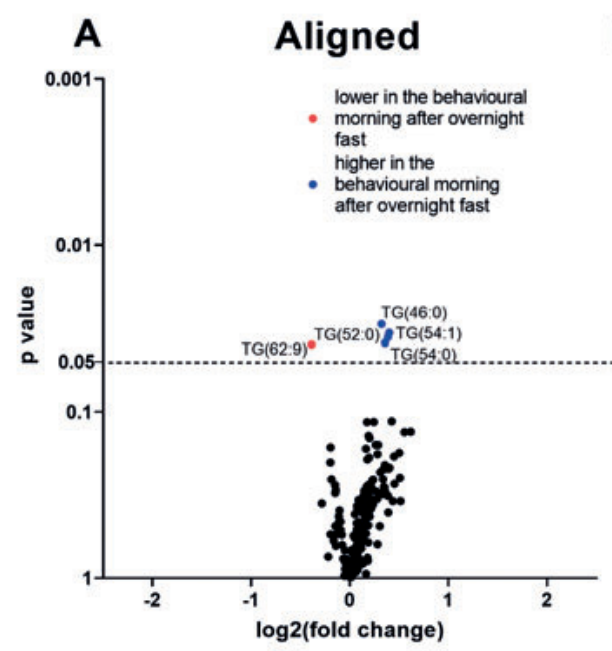

B Misaligned

Figure 4. Volcano plot for triacylglycerols (TG) without any hydroxy [M+OH] and dihydroxy/peroxy species $[\mathrm{M}+\mathrm{OOH}]$ within the aligned condition $(A)$ and the misaligned condition (B). Note that in the misaligned condition most TGs scatter to the right indicating that TGs were consistently higher in the behavioural morning at 8 PM. Also, within the misaligned condition, the significantly higher TG species (blue dots) consisted exclusively of $\geq 55$ carbons. The exact log2(fold change) values for each individual TG species that was significantly altered can be extracted from supplemental Tables S1 and S2. 


\section{Lipid classes show distinct response patterns to misalignment}

Next, we aimed to investigate if a lipid class was affected by circadian misalignment (example in Figure 5A) or if it continues to follow clock time (example in Figure 5B) - likely reflecting rhythmicity induced by the biological clock. A third possibility of how the skeletal muscle lipidome could respond to circadian misalignment is that it adjusts to the new behaviour, for example if certain lipid classes are lower after the overnight fast compared with the fed state in the aligned condition, this difference should persist upon misalignment irrespective of clock time (example in Figure 5C). Thus, by two-way repeated measures ANOVAs we tested for time (8 AM vs 8 PM; clock-driven), condition (aligned vs misaligned), and/or interaction (may follow behaviour) effects. This analysis revealed a significant time effect for phosphatidylcholines (PC, $\mathrm{p}=0.047$, Figure 5D), suggesting that $\mathrm{PC}$ are higher at 8 PM irrespective of behaviour supporting the notion that these lipid classes are subjected to the control of the intrinsic skeletal muscle clock. Lipid classes for which a significant condition effect was found were the cardiolipins ( $C L, p=0.022$, Figure 5E), with the most abundant species CL(72:8) (reflecting $~ 85 \%$ of the total sum of detected $\mathrm{CL}, \mathrm{p}=0.029$; Figure 5J) and monolysocardiolipin (MLCL) $(\mathrm{p}=0.025)$ driving the effect, and a trend for increased lysophosphatidylethanolamines (LPE) upon misalignment $(\mathrm{p}=0.087$, Figure 5F). Although CL(72:8) and MLCL did not show morning-evening differences, their levels were generally lower upon circadian misalignment, suggesting that they are negatively affected by misalignment, which could be due to unanticipated food intake and/or physical activity. Finally, a significant interaction effect was found for cholesteryl esters (CE, p=0.044, Figure 5G), suggesting that this lipid class rapidly adapts to the new behaviour after the rapid day-night shift. This effect seemed to be primarily driven by the levels of the most abundant CE species (cholesteryl docosahexaenoic acid (CE(22:6) reflecting $~ 70 \%$ on average of the total sum of detected CE, p=0.027; Figure 5K). For hexosylceramides (HexCer[d], Figure 5H) and ceramide phosphocholines (SM[d], Figure 5I), we found a tendency towards an interaction effect (both: $\mathrm{p}=0.056$ ). None of the altered lipid classes/species was significantly correlated to skeletal muscle insulin sensitivity, neither when considering the absolute nor the \%change values between the two clamps. 
1. Example patterns of effects detected by the 2way ANOVA:

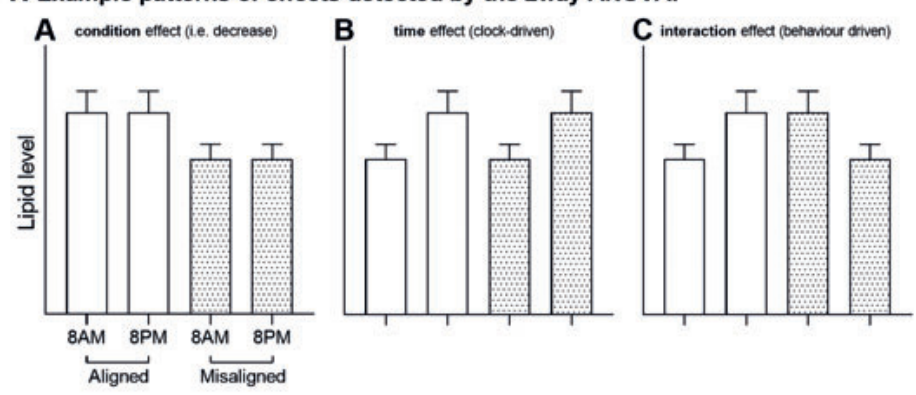

2. Overview of lipid classes with a time, condition or interaction of $p<0.1$ :
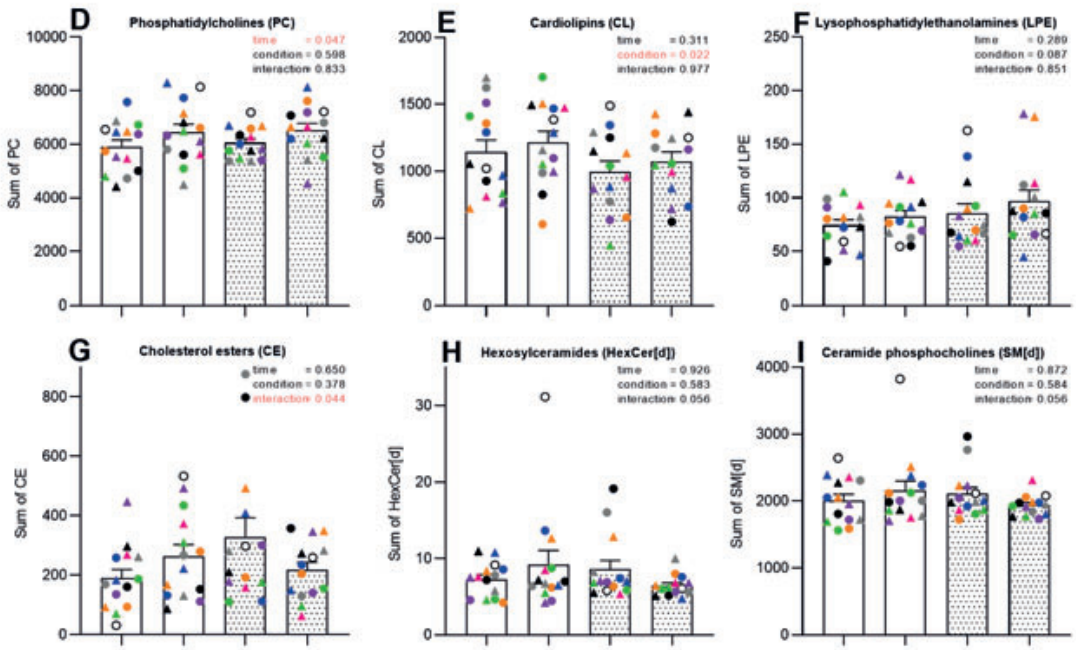

3. Most abundant lipid species within the lipid classes with an effect of $p<0.05$ :
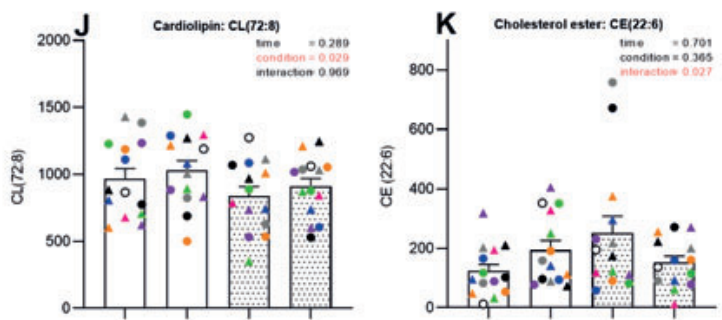

Figure 5. Lipid classes upon circadian misalignment. (1) Example patterns of the effects detected by the two-way repeated measures ANOVA (time, condition and interaction). (2) Overview of results for the sums of selected lipid classes (any effect of $p<0.1$ ) without any hydroxy $[\mathrm{M}+\mathrm{OH}]$ and dihydroxy/ peroxy species $[\mathrm{M}+\mathrm{OOH}]$. Each coloured icon represents the total sum of the respective lipid class of an individual participant. Data are displayed as mean \pm SEM $(n=14)$. (3) For those lipid classes, in which the ANOVA revealed a significant effect, the most abundant species are shown. Note that except for the phospatidylcholines (PC), the effect within the lipid class is strongly driven by the level of the most abundant species. Significant results $(p<0.05)$ are highlighted in red. Other detected lipid classes that were unaffected by misalignment are shown in supplemental Figure S1. 


\section{Discussion}

We recently demonstrated that a rapid day-night shift introduced skeletal muscle insulin resistance, when measured after an "overnight" fast at 8 PM. In the here presented follow-up study, we performed detailed analyses of skeletal muscle LD morphology and the muscle lipidome to investigate if this misalignmentinduced insulin resistance was paralleled by an altered lipid metabolism. We could demonstrate that short-term circadian misalignment was not associated with changes in intramyocellular LD characteristics but did result in alterations in the muscle lipidome, which were however not directly related to changes in insulin sensitivity.

For the LD characteristics, we observed no changes in the muscle biopsies taken in the aligned (8 AM) and misaligned (8 PM) condition, which therefore cannot explain the reductions in insulin sensitivity observed between these time points. It could be that the misalignment protocol was too short to result in detectable changes in lipid content, and it should be acknowledged that we tested lean healthy young men who are known to have relatively low levels of lipid content (Goodpaster et al., 2001; Daemen et al., 2018). Using a similar analysis approach, we previously found day-night rhythmicity in LD number and LD size in skeletal muscle biopsies from comparably healthy young men, especially in type I muscle fibres (Held et al., 2020). However, the clock times at which biopsies were taken in the present study (8 AM vs $8 \mathrm{PM}$ ) might have influenced the outcome. In this regard, our current findings are in line with our previous study, in which we demonstrated that lipid droplet characteristics were relatively stable at the respectively closest time points (8 AM and 6 PM in (Held et al., 2020)) and likewise intramyocellular lipid content determined by ${ }^{1} \mathrm{H}-\mathrm{MRS}$ in lower leg muscles was stable at similar clock times (Machann et al., 2011).

Whereas the lipidome showed minimal morning-evening (8 AM vs 8 PM) differences under aligned conditions (only 19 out of 1171 detected lipids), $\sim 5$ times more lipids (101 out of 1171) displayed a morning-evening difference upon misalignment. Most lipids that changed between morning and evening upon misalignment were triradylglycerols. More specifically, polyunsaturated triradylglycerol species of a 


\section{Chapter 4}

carbon length $\geq 55$ were more abundant at $8 \mathrm{PM}$ in the fasted state (the behavioural morning) upon misalignment likely reflecting a higher degree of incorporation of long-chain or very long-chain polyunsaturated fatty acids in these lipids. This finding could point to a preferable oxidation of short-chain fatty acids and/or fatty acid elongation. Interestingly, we previously showed that one of the enriched gene sets when comparing the 8 AM with 8 PM biopsies in the misaligned condition was related to fatty acid elongation (Wefers et al., 2018). Taken together, these findings suggest that circadian misalignment impacts on the process of fatty acid elongation, resulting in long-chain, polyunsaturated triradylglycerol species as observed in the current study. In this context, it is also interesting to note that elongases (involved in elongation of very long-chain fatty acid) can be either rhythmically expressed or impaired upon disruption of clock genes (Zhang et al., 2014; Hodge et al., 2015; Loizides-Mangold et al., 2017). Furthermore, in contrast to fatty acids with shorter acyl chains, fatty acids with longer chain length contribute to insulin resistance, that is 10-12 vs 16-18 carbons (Hommelberg et al., 2009; Montgomery et al., 2013), which hints at a link between the muscle triradylglycerol profile and the skeletal muscle insulin resistance, observed upon misalignment. However, exploratory correlation analysis between long-chain, polyunsaturated triradylglycerol levels, and muscle insulin sensitivity did not reveal significant associations.

We recently demonstrated 24-hour rhythmicity of the skeletal muscle lipidome by performing lipidomics in muscle biopsies taken from lean, healthy subjects around the clock (Held et al., 2020). However, in that study, volunteers followed a normal 24-hour day-night pattern, including consumption of three meals a day and some physical activity in between; therefore, it could not be concluded if the rhythmicity in lipid species was driven by the circadian clock or by the 24-hour behaviour of the volunteers. As the current study design allowed us to better distinguish between these two factors, we used two-way repeated measures ANOVAs on the current muscle lipidomics dataset to test for time (which would suggest regulation by the circadian clock), condition (suggesting a specific effect of misalignment) and/or interaction (suggesting regulation by behaviour) effects. The latter interaction effect can imply 
that certain lipid classes might strictly adjust to the times of food intake and/or physical activity and would hence display different levels in the fasted behavioural morning biopsy (after sleep) than in the fed behavioural evening biopsy (after some physical activity over the course of the respective daytime). PC, the only lipid class with a significant time effect, did not adjust to the new behaviour reinforced by the rapid shift and hence inverted times of food intake and physical activity, suggesting that PC mainly follow the control of the molecular core clock in skeletal muscle. Indeed, we previously observed that the expression of core clock components in skeletal muscle was not inverted in line with the shifted behaviour (Wefers et al., 2018). Consistently, silencing of the Clock gene in human primary myotubes lowers the amplitude of circadian rhythmicity in PC (Loizides-Mangold et al., 2017). We previously showed that several PC species showed 24-hour rhythmicity in humans (Held et al., 2020) and the current results suggest that their 24-hour rhythmicity is indeed most likely driven by the circadian clock.

The dimeric phospholipid cardiolipin (CL) was the only lipid class that showed a condition effect, evidenced by lower levels upon misalignment independent of morning or evening. CL is an essential component of the mitochondrial inner membrane regulating the activity and stability of numerous membrane-bound protein complexes and the electron transport chain (Houtkooper \& Vaz, 2008; Kameoka et al., 2018). Lower CL levels are often considered a reflection of reduced mitochondrial mass and functioning (Houtkooper \& Vaz, 2008). However, circadian misalignment did not induce changes in mitochondrial respiration upon a lipid substrate and even a higher mitochondrial respiration upon pyruvate as a substrate (Wefers et al., 2018). It could be speculated that more persistent, long-term reductions in CL would ultimately also result in mitochondrial dysfunction upon misalignment; however, this cannot be deduced from the current data. For some cholesteryl esters (CE), we previously showed 24-hour rhythmicity in skeletal muscle peaking in the early evening (Held et al., 2020). In the current study, CE was the only lipid class for which a significant interaction effect was found. Accordingly, CE seem to follow the shifted behaviour instead of being generally lower or higher with misalignment, suggesting 


\section{Chapter 4}

that CE strongly respond to the new behaviour and are hence rather independent of the molecular clock. Again, exploratory correlation analysis between these species and muscle insulin sensitivity did not reveal significant associations, suggesting that none of the identified lipids can be directly linked to the misalignment-induced insulin resistance.

Other factors may hence play a greater role in the induction of insulin resistance, such as plasma free fatty acids and core temperature that were both elevated in the behavioural morning upon misalignment (Wefers et al., 2018). The contribution of misalignment-associated sleep deprivation should also not be underestimated, as it leads to increased catecholamine levels (Grimaldi et al., 2016), which could affect muscle insulin sensitivity by upregulating lipolysis. Alternatively, the 24-hour rhythm in cortisol has been shown to be reversed upon circadian misalignment (Scheer et al., 2009), suggesting that atypically low cortisol levels in the behavioural morning are involved in the muscle insulin resistance detected at this time of the day. However, investigation of these hypotheses was beyond the scope of the present study, as the scope of this study was to investigate the impact of circadian misalignment on the muscle lipidome and lipid droplet morphology.

One limitation of the current study, inherent to performing such experiments in humans, is the fact that we had limited number of time points on which muscle biopsies were sampled. As our muscle biopsies were targeted to be in conjunction with the hyperinsulinemic-euglycemic clamp to determine insulin sensitivity, biopsies were taken at $8 \mathrm{AM}$ and $8 \mathrm{PM}$ in the aligned and misaligned condition, respectively. To further detect morning-evening differences, we planned the two other biopsies at $8 \mathrm{PM}$ and $8 \mathrm{AM}$ in the aligned and misaligned arm, respectively. However, in our previous study on 24-hour rhythmicity in the skeletal muscle lipidome (Held et al., 2020), which was performed over the same period as the current experiment, we found that most lipids were only increased late in the night in biopsies taken at $4 \mathrm{AM}$ after subjects were asleep, whereas at 8 AM, 1 PM, 6 PM and 11 PM only few lipid species were altered. Similarly, the observation of Loizides-Mangold et al. (LoizidesMangold et al., 2017) that $\sim 20 \%$ of the human muscle lipidome was rhythmic was 
based on six muscle biopsies performed over 24 hours with most lipids peaking during the night. Therefore, our current findings are to some extent limited to those lipid species that did show variance between morning (8 AM) and evening (8 PM). We suspect that by performing a biopsy at the middle of the respective sleeping period, future studies may reveal many more lipid classes to be disrupted upon circadian misalignment.

In conclusion, we demonstrated that controlled short-term circadian misalignment disturbed the skeletal muscle lipidome, whereas lipid droplet morphology remained unaltered. We were able to distinguish between lipid classes that strongly followed the intrinsic skeletal muscle clock, lipid classes that were generally lowered upon misalignment and lipid classes that adjusted to the new behaviour. Future studies should assess mechanisms how these identified lipid classes may contribute to the acutely observed skeletal muscle insulin resistance and chronically to the increased risk to develop T2DM upon repetitive shift work. 


\section{Supplementary data for chapter 4}

Table S1. Paired t-tests results for the significantly altered lipid species (19) within the aligned condition: 8PM (behavioural evening) vs 8AM (behavioural morning).

\begin{tabular}{lll}
\hline Lipid species & p-value & Log2 fold change \\
\hline TG(O-46:11) & 0.007068 & -0.406509 \\
Cer(d46:1) [M+OH]+ & 0.013488 & -0.490473 \\
TG(40:1) [M+OOH]+ & 0.015383 & -0.281409 \\
PC(O-32:1) [M+OH]+ [M+OH]+ & 0.021285 & -0.457143 \\
PC(38:5) & 0.024064 & -0.20152 \\
Cer(d45:1) [M+OOH]+ & 0.025785 & -0.470307 \\
DG(39:3) [M+OOH]+ & 0.027639 & -0.448694 \\
Cer(d45:2) [M+OH]+ & 0.029374 & -0.331642 \\
TG(46:0) & 0.029775 & 0.32239 \\
TG(54:1) & 0.033601 & 0.40107 \\
TG(52:0) & 0.035651 & 0.38677 \\
PC(38:7) & 0.037651 & -0.306493 \\
TG(54:0) & 0.038526 & 0.35828 \\
TG(62:9) & 0.039466 & -0.387008 \\
Hex2Cer(d36:2) & 0.04249 & -0.703925 \\
Cer(d40:0) & 0.043442 & -0.266857 \\
TG(58:2) $[\mathrm{M}+\mathrm{OH}]+$ & 0.044555 & 0.34338 \\
Cer(d38:0) & 0.046973 & -0.342206 \\
Cer(d45:1) & 0.048261 & -0.223589 \\
\hline
\end{tabular}

Positive log 2 fold change value indicates a higher level of the respective lipid in the behavioural morning ( $8 A M)$ and a negative value a higher value in the behavioural evening (8PM). 
Table S2. Paired t-tests results for the significantly altered lipid species (101) within the misaligned condition: 8AM (behavioural evening) vs 8PM (behavioural morning).

\begin{tabular}{|c|c|c|}
\hline Lipid species & $\mathrm{p}$-value & Log2 fold change \\
\hline $\mathrm{TG}(\mathrm{O}-64: 9)[\mathrm{M}+\mathrm{OH}]+$ & 0.001159 & 0.561476 \\
\hline TG(O-64:8) [M+OH]+ & 0.002207 & 0.592007 \\
\hline $\mathrm{TG}(\mathrm{O}-62: 8)[\mathrm{M}+\mathrm{OH}]+$ & 0.003108 & 0.543794 \\
\hline $\mathrm{PC}(35: 1)$ & 0.003151 & 0.288173 \\
\hline $\mathrm{PC}(\mathrm{O}-36: 3)$ & 0.003339 & 0.426799 \\
\hline TG(O-66:7) [M+OH]+ & 0.003542 & 0.557565 \\
\hline $\mathrm{PC}(\mathrm{O}-36: 2)$ & 0.003631 & 0.427869 \\
\hline $\mathrm{TG}(\mathrm{O}-64: 10)[\mathrm{M}+\mathrm{OH}]+$ & 0.003859 & 0.565825 \\
\hline HexCer(d26:1) [M+OH]+ & 0.004172 & 0.428041 \\
\hline TG(64:6) & 0.004293 & 0.637622 \\
\hline TG(57:9) & 0.004442 & 0.406319 \\
\hline TG(58:8) & 0.004486 & 0.27737 \\
\hline PC(37:2) & 0.005194 & 0.314159 \\
\hline $\mathrm{TG}(58: 7)$ & 0.005245 & 0.356976 \\
\hline $\mathrm{TG}(\mathrm{O}-62: 9)[\mathrm{M}+\mathrm{OH}]+$ & 0.006182 & 0.426509 \\
\hline TG(O-66:9) [M+OH]+ & 0.006528 & 0.682719 \\
\hline $\mathrm{PC}(\mathrm{O}-34: 2)$ & 0.007226 & 0.3828 \\
\hline $\mathrm{TG}(\mathrm{O}-46: 11)$ & 0.00725 & 0.376956 \\
\hline DG(24:0) [M+OOH]+ & 0.008662 & 0.197656 \\
\hline $\mathrm{TG}(\mathrm{O}-29: 0)$ & 0.00869 & 0.344235 \\
\hline $\mathrm{TG}(62: 6)$ & 0.008988 & 0.531238 \\
\hline TG(60:6) & 0.009517 & 0.535812 \\
\hline $\mathrm{PC}(36: 1)$ & 0.010508 & 0.277027 \\
\hline $\mathrm{SM}(\mathrm{d} 44: 5)$ & 0.01083 & -0.21913 \\
\hline $\mathrm{PC}(\mathrm{O}-33: 2)$ & 0.011225 & 0.634857 \\
\hline $\mathrm{PC}(\mathrm{O}-34: 3)$ & 0.011311 & 0.3079 \\
\hline $\mathrm{SM}(\mathrm{d} 42: 1)[\mathrm{M}+\mathrm{OOH}]+$ & 0.012557 & -0.90774 \\
\hline $\mathrm{PC}(\mathrm{O}-38: 3)$ & 0.013771 & 0.239525 \\
\hline $\mathrm{SM}(\mathrm{t} 40: 1)$ & 0.014501 & -0.43632 \\
\hline TG(63:7) & 0.015187 & 0.745415 \\
\hline $\mathrm{PC}(\mathrm{O}-35: 3)$ & 0.015475 & 0.363409 \\
\hline $\mathrm{CE}(17: 1)[\mathrm{M}+\mathrm{OOH}]+$ & 0.015573 & 0.377651 \\
\hline $\mathrm{PC}(\mathrm{O}-40: 3)$ & 0.015592 & 0.384571 \\
\hline DG(O-42:5) & 0.016037 & 0.262092 \\
\hline $\mathrm{TG}(60: 7)$ & 0.016137 & 0.414931 \\
\hline
\end{tabular}


Chapter 4

\begin{tabular}{|c|c|c|}
\hline $\operatorname{SM}(\mathrm{t} 42: 2)$ & 0.017437 & -0.47059 \\
\hline TG(59:5) & 0.019202 & 0.536089 \\
\hline TG(O-33:0) & 0.019664 & 0.157531 \\
\hline TG(57:4) & 0.019895 & 0.47138 \\
\hline $\mathrm{PC}(35: 3)$ & 0.020007 & 0.252522 \\
\hline TG(60:5) & 0.020516 & 0.519396 \\
\hline Cer(d40:3) & 0.020792 & -0.48846 \\
\hline TG(61:6) & 0.021108 & 0.835651 \\
\hline TG(58:6) & 0.021284 & 0.397931 \\
\hline $\mathrm{SM}(\mathrm{d} 44: 4)$ & 0.021414 & -0.14707 \\
\hline $\mathrm{PC}(36: 2)$ & 0.022341 & 0.181966 \\
\hline $\mathrm{SM}(\mathrm{d} 30: 0)$ & 0.023033 & 0.180219 \\
\hline DG(24:0) & 0.023532 & 0.228403 \\
\hline TG(O-30:0) & 0.024207 & 0.240898 \\
\hline Cer(d38:2) & 0.025317 & -0.38003 \\
\hline PC(O-36:4) & 0.025737 & 0.326377 \\
\hline TG(55:4) & 0.025761 & 0.369069 \\
\hline $\mathrm{TG}(57: 7)$ & 0.025831 & 0.420054 \\
\hline $\mathrm{SPH}(18: 1)[\mathrm{M}+\mathrm{OOH}]+$ & 0.026318 & 0.197844 \\
\hline PC(37:3) & 0.026356 & 0.213629 \\
\hline PC(35:2) & 0.027136 & 0.23441 \\
\hline TG(59:4) & 0.027301 & 0.585517 \\
\hline TG(63:6) & 0.027629 & 0.672101 \\
\hline $\mathrm{TG}(46: 1)[\mathrm{M}+\mathrm{OH}]+$ & 0.027643 & 0.227709 \\
\hline TG(63:5) & 0.027802 & 0.529947 \\
\hline $\operatorname{Cer}(\mathrm{d} 48: 1)[\mathrm{M}+\mathrm{OH}]+$ & 0.029465 & 0.351198 \\
\hline $\mathrm{SM}(\mathrm{d} 42: 1)$ & 0.030105 & -0.18088 \\
\hline LPC(12:0) & 0.031799 & 0.203058 \\
\hline PC $(34: 3)$ & 0.031825 & 0.222476 \\
\hline TG(61:5) & 0.032836 & 0.644535 \\
\hline TG(O-34:0) & 0.033146 & 0.198953 \\
\hline $\mathrm{SM}(\mathrm{t} 36: 1)$ & 0.033193 & -0.49184 \\
\hline TG(55:6) & 0.033202 & 0.348483 \\
\hline PE(42:10) & 0.033328 & 0.250551 \\
\hline PC $(26: 0)$ & 0.033559 & 0.20159 \\
\hline TG(58:0) & 0.033928 & 0.350797 \\
\hline TG(56:6) & 0.034217 & 0.27379 \\
\hline
\end{tabular}


Circadian misalignment and muscle lipidome

\begin{tabular}{|c|c|c|}
\hline TG(60:8) & 0.034387 & 0.329366 \\
\hline $\operatorname{SM}(\mathrm{d} 44: 6)$ & 0.034484 & -0.28324 \\
\hline $\mathrm{TG}(58: 5)$ & 0.035033 & 0.446046 \\
\hline Cer(d44:0) [M+OH]+ & 0.036537 & 0.356549 \\
\hline $\mathrm{TG}(57: 6)$ & 0.037484 & 0.433101 \\
\hline $\mathrm{PC}(36: 3)$ & 0.037545 & 0.190611 \\
\hline TG(62:5) & 0.038227 & 0.463687 \\
\hline $\mathrm{SPH}(18: 0)[\mathrm{M}+\mathrm{OH}]^{+}$ & 0.03887 & 0.251579 \\
\hline TG(61:6) & 0.040193 & 0.576546 \\
\hline TG(O-61:4) [M+OH]+ & 0.040706 & 0.593148 \\
\hline TG(48:0) [M+OOH]+ & 0.041131 & 0.289932 \\
\hline TG(55:5) & 0.041765 & 0.322496 \\
\hline TG(59:7) & 0.042026 & 0.545196 \\
\hline $\mathrm{TG}(56: 2)[\mathrm{M}+\mathrm{OH}]+$ & 0.042222 & 0.239013 \\
\hline TG(59:3) & 0.042712 & 0.643149 \\
\hline TG(O-58:9) & 0.042803 & 0.377352 \\
\hline $\mathrm{PC}(\mathrm{O}-34: 4)$ & 0.042903 & 0.262585 \\
\hline $\mathrm{TG}(57: 8)$ & 0.043583 & 0.287926 \\
\hline Cer(d45:1) & 0.044612 & 0.141789 \\
\hline HexCer(d28:1) [M+OOH]+ & 0.04596 & 0.347986 \\
\hline $\mathrm{PC}(33: 1)$ & 0.046222 & 0.252839 \\
\hline $\mathrm{TG}(60: 4)$ & 0.046899 & 0.522898 \\
\hline TG(59:2) & 0.046985 & 0.546224 \\
\hline TG(59:4) & 0.047622 & 0.609455 \\
\hline $\mathrm{PC}(\mathrm{O}-38: 6)[\mathrm{M}+\mathrm{OH}]+[\mathrm{M}+\mathrm{OH}]+$ & 0.047963 & -0.48041 \\
\hline Cer(d43:0) [M+OH]+ & 0.048984 & 0.288979 \\
\hline TG(57:4) & 0.049531 & 0.568773 \\
\hline TG(56:7) & 0.049926 & 0.244178 \\
\hline TG(63:4) & 0.049991 & 0.375153 \\
\hline
\end{tabular}

Positive log2 fold change value indicates a higher level of the respective lipid in the behavioural morning ( $8 P M)$ and a negative value a higher value in the behavioural evening ( $8 A M)$. 

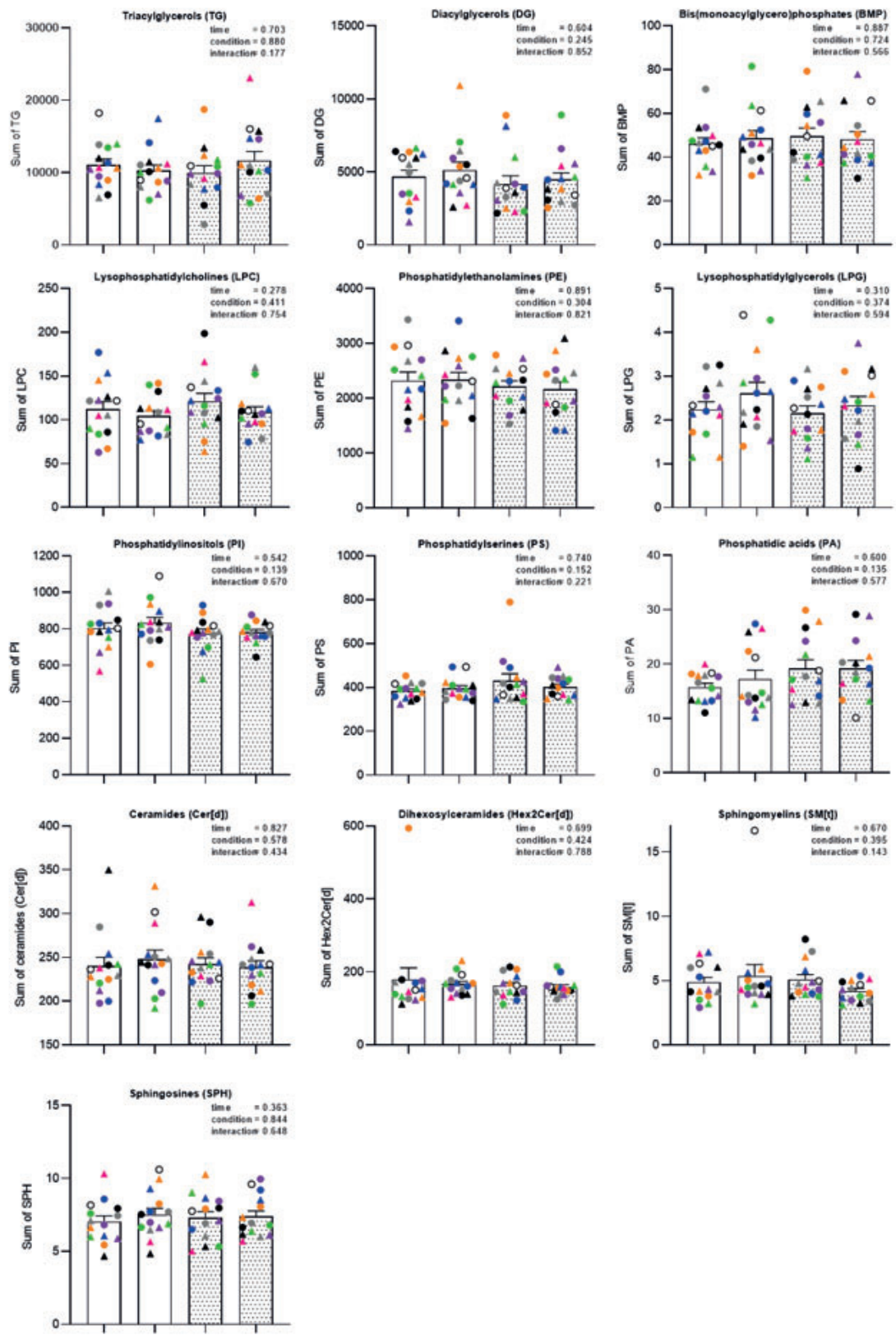

Figure S1. Overview of results for the sums of other lipid classes without any hydroxy [M+OH] and dihydroxy/peroxy species [M+OOH]. Each coloured icon represents the total sum of the respective lipid class of an individual participant. Data are displayed as mean \pm SEM $(n=14)$. 


\section{References}

Amati F, Dubé JJ, Alvarez-Carnero E, Edreira MM, Chomentowski P, Coen PM, Switzer GE, Bickel PE, Stefanovic-Racic M, Toledo FGS \& Goodpaster BH. (2011). Skeletal muscle triglycerides, diacylglycerols, and ceramides in insulin resistance: another paradox in endurance-trained athletes? Diabetes 60, 2588-2597.

Covington JD, Johannsen DL, Coen PM, Burk DH, Obanda DN, Ebenezer PJ, Tam CS, Goodpaster BH, Ravussin E \& Bajpeyi S. (2017). Intramyocellular Lipid Droplet Size Rather Than Total Lipid Content is Related to Insulin Sensitivity After 8 Weeks of Overfeeding. Obesity 25, 2079-2087.

Daemen S, Gemmink A, Brouwers B, Meex RCR, Huntjens PR, Schaart G, Moonen-Kornips E, Jörgensen J, Hoeks J, Schrauwen P \& Hesselink MKC. (2018). Distinct lipid droplet characteristics and distribution unmask the apparent contradiction of the athlete's paradox. Mol Metab 17, 71-81.

DeFronzo RA \& Tripathy D. (2009). Skeletal muscle insulin resistance is the primary defect in type 2 diabetes. Diabetes Care 32 Suppl 2, S157-163.

Gemmink A, Goodpaster BH, Schrauwen P \& Hesselink MKC. (2017). Intramyocellular lipid droplets and insulin sensitivity, the human perspective. Biochim Biophys Acta Mol Cell Biol Lipids 1862, 1242-1249.

Goodpaster BH, He J, Watkins S \& Kelley DE. (2001). Skeletal muscle lipid content and insulin resistance: evidence for a paradox in endurance-trained athletes. J Clin Endocrinol Metab 86, 5755-5761.

Grimaldi D, Carter JR, Van Cauter E \& Leproult R. (2016). Adverse Impact of Sleep Restriction and Circadian Misalignment on Autonomic Function in Healthy Young Adults. Hypertension 68, 243-250.

Gutierrez-Monreal MA, Harmsen JF, Schrauwen P \& Esser KA. (2020). Ticking for Metabolic Health: The Skeletal-Muscle Clocks. Obesity (Silver Spring) 28 Suppl 1, S46-s54.

Held NM, Wefers J, van Weeghel M, Daemen S, Hansen J, Vaz FM, van Moorsel D, Hesselink MKC, Houtkooper RH \& Schrauwen P. (2020). Skeletal muscle in healthy humans exhibits a day-night rhythm in lipid metabolism. Mol Metab 37, 100989.

Herzog K, Pras-Raves ML, Vervaart MAT, Luyf ACM, van Kampen AHC, Wanders RJA, Waterham HR \& Vaz FM. (2016). Lipidomic analysis of fibroblasts from Zellweger spectrum disorder patients identifies disease-specific phospholipid ratios. J Lipid Res 57, 1447-1454.

Hodge BA, Wen Y, Riley LA, Zhang X, England JH, Harfmann BD, Schroder EA \& Esser KA. (2015). The endogenous molecular clock orchestrates the temporal separation of substrate metabolism in skeletal muscle. Skelet Muscle 5, 17.

Hommelberg PPH, Plat J, Langen RCJ, Schols AMWJ \& Mensink RP. (2009). Fatty acidinduced NF-kappaB activation and insulin resistance in skeletal muscle are chain length dependent. Am J Physiol Endocrinol Metab 296, E114-120.

Houtkooper RH \& Vaz FM. (2008). Cardiolipin, the heart of mitochondrial metabolism. Cell Mol Life Sci 65, 2493-2506.

Kameoka S, Adachi Y, Okamoto K, Iijima M \& Sesaki H. (2018). Phosphatidic Acid and Cardiolipin Coordinate Mitochondrial Dynamics. Trends Cell Biol 28, 67-76.

Koshy A, Cuesta M, Boudreau P, Cermakian N \& Boivin DB. (2019). Disruption of central and peripheral circadian clocks in police officers working at night. Faseb j 33, 6789-6800. 


\section{Chapter 4}

Loizides-Mangold U, Perrin L, Vandereycken B, Betts JA, Walhin J-P, Templeman I, Chanon S, Weger BD, Durand C, Robert M, Paz Montoya J, Moniatte M, Karagounis LG, Johnston JD, Gachon F, Lefai E, Riezman H \& Dibner C. (2017). Lipidomics reveals diurnal lipid oscillations in human skeletal muscle persisting in cellular myotubes cultured in vitro. Proc Natl Acad Sci U S A 114, E8565-E8574.

Machann J, Etzel M, Thamer C, Haring H-U, Claussen CD, Fritsche A \& Schick F. (2011). Morning to evening changes of intramyocellular lipid content in dependence on nutrition and physical activity during one single day: a volume selective ${ }^{1} \mathrm{H}-\mathrm{MRS}$ study. MAGMA 24, 29-33.

Montgomery MK, Osborne B, Brown SHJ, Small L, Mitchell TW, Cooney GJ \& Turner N. (2013). Contrasting metabolic effects of medium- versus long-chain fatty acids in skeletal muscle. J Lipid Res 54, 3322-3333.

Nielsen J, Christensen AE, Nellemann B \& Christensen B. (2017). Lipid droplet size and location in human skeletal muscle fibers are associated with insulin sensitivity. Am J Physiol Endocrinol Metab 313, E721-E730.

Saad A, Dalla Man C, Nandy DK, Levine JA, Bharucha AE, Rizza RA, Basu R, Carter RE, Cobelli C, Kudva YC \& Basu A. (2012). Diurnal pattern to insulin secretion and insulin action in healthy individuals. Diabetes 61, 2691-2700.

Scheer FA, Hilton MF, Mantzoros CS \& Shea SA. (2009). Adverse metabolic and cardiovascular consequences of circadian misalignment. Proc Natl Acad Sci U S A 106, 4453-4458.

Sulli G, Manoogian ENC, Taub PR \& Panda S. (2018). Training the Circadian Clock, Clocking the Drugs, and Drugging the Clock to Prevent, Manage, and Treat Chronic Diseases. Trends Pharmacol Sci 39, 812-827.

Van Cauter E, Blackman JD, Roland D, Spire JP, Refetoff S \& Polonsky KS. (1991). Modulation of glucose regulation and insulin secretion by circadian rhythmicity and sleep. J Clin Invest 88, 934-942.

Wefers J, van Moorsel D, Hansen J, Connell NJ, Havekes B, Hoeks J, van Marken Lichtenbelt WD, Duez H, Phielix E, Kalsbeek A, Boekschoten MV, Hooiveld GJ, Hesselink MKC, Kersten S, Staels B, Scheer F \& Schrauwen P. (2018). Circadian misalignment induces fatty acid metabolism gene profiles and compromises insulin sensitivity in human skeletal muscle. Proc Natl Acad Sci U S A 115, 7789-7794.

Zhang R, Lahens NF, Ballance HI, Hughes ME \& Hogenesch JB. (2014). A circadian gene expression atlas in mammals: implications for biology and medicine. Proc Natl Acad Sci U S A 111, 16219-16224. 
Circadian misalignment and muscle lipidome 


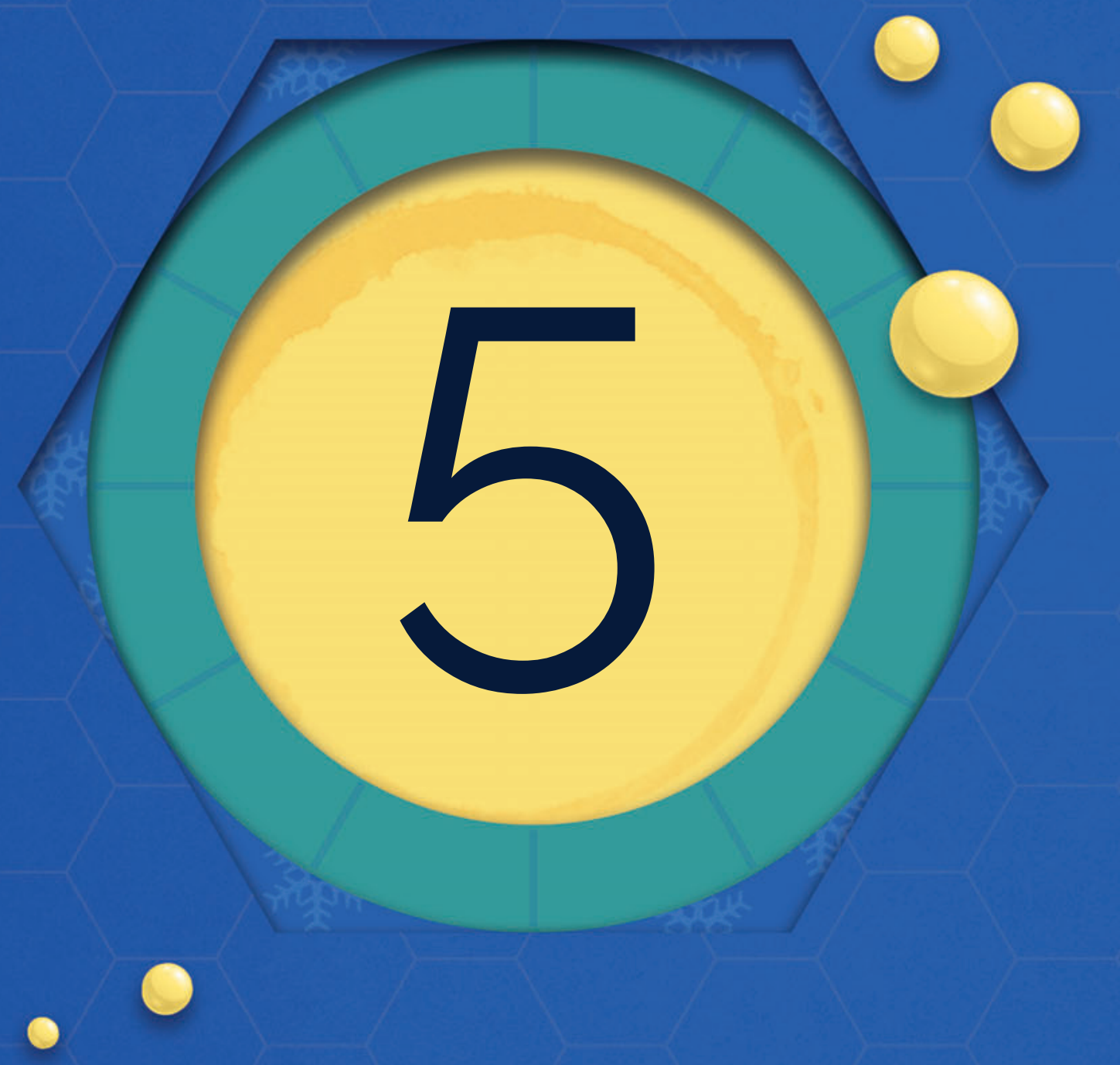




\section{Resveratrol-induced remodelling of myocellular lipid stores: a study in metabolically compromised humans}

Nynke van Polanen, Evelyn Zacharewicz, Marlies de Ligt, Silvie

Timmers, Esther Moonen-Kornips, Gert Schaart, Joris Hoeks, Patrick

Schrauwen, Matthijs K.C. Hesselink

Published in Physiological Reports 2021 Jan; 9(2): e14692 


\section{Chapter 5}

\section{Abstract}

In non-athletes, insulin sensitivity correlates negatively with intramyocellular lipid (IMCL) content. In athletes, however, a pattern of benign IMCL storage exists, which is characterized by lipid storage in type I muscle fibres, in small and numerous lipid droplets (LDs) preferable coated with PLIN5, without affecting insulin sensitivity. Administration of resveratrol has been promoted for its beneficial effects on glucose homeostasis. We observed that 30 days of oral resveratrol administration $(150 \mathrm{mg} /$ day) in metabolically compromised individuals showed a $33 \%$ increase in IMCL (placebo vs resveratrol; $0.86 \pm 0.090 \mathrm{AU}$ vs $1.14 \pm 0.11 \mathrm{AU}, \mathrm{p}=0.003$ ) without impeding insulin sensitivity. Thus, the aim of the present study was to examine if a resveratrolmediated increase in IMCL content, in metabolically compromised individuals, changes the LD phenotype towards the phenotype we previously observed in athletes. For this, we studied IMCL, LD number, LD size, subcellular distribution and PLIN5 coating in different fibre types using high-resolution confocal microscopy. As proof of concept, we observed a 2.3-fold increase $(\mathrm{p}=0.038)$ in lipid accumulation after 48 hours of resveratrol incubation in cultured human primary muscle cells. In vivo analysis showed that resveratrol-induced increase in IMCL is predominantly in type I muscle fibres (placebo vs resveratrol; $0.97 \pm 0.16 \%$ vs $1.26 \pm 0.09 \%$; $=0.030$ ) in both the subsarcolemmal $(\mathrm{p}=0.016)$ and intermyofibrillar region $(\mathrm{p}=0.026)$ and particularly in PLIN5 coated LDs $(\mathrm{p}=0.024)$. These data indicate that administration of resveratrol augments IMCL content in metabolically compromised individuals towards a LD phenotype that mimics an 'athlete like phenotype'. 


\section{Introduction}

Compromised insulin-mediated glucose uptake in muscle is commonly observed in middle-aged, overweight individuals and is a hallmark in the pathogenesis of insulin resistance and type 2 diabetes mellitus (T2DM) development (DeFronzo \& Tripathy, 2009). In sedentary populations, there is a negative association between insulin sensitivity and intramyocellular lipid (IMCL) storage (Pan et al., 1997; Krssak et al., 1999; Goodpaster et al., 2000). Paradoxically, trained athletes with similarly high IMCL levels as patients with T2DM are highly insulin-sensitive (Goodpaster et al., 2001). This paradox appears to originate from differences in lipid composition (Metcalfe et al., 2019), lipid morphology (Nielsen et al., 2017; Daemen et al., 2018) and/ or subcellular distribution of lipid droplets (LDs) within the muscle (Daemen et al., 2018; Perreault et al., 2018).

To alleviate insulin resistance and its metabolic consequences, caloric restriction and physical activity are among the most powerful interventions. However, long-term adherence to these interventions is low (Middleton et al., 2013). This has triggered the search for drug or nutritional interventions that mimic the effects of caloric restriction and/or exercise. In this respect, the polyphenol resveratrol is of interest as it promotes mitochondrial function and metabolic health in model systems via SIRT1 and PGC1 $\alpha$ (Lagouge et al., 2006). In rodents, resveratrol blunted high-fat diet-induced hepatic lipid storage (Alberdi et al., 2013; Zhou et al., 2018), muscle lipid storage (Chen et al., 2011; Zhang et al., 2019; Gong et al., 2020) and improved insulin sensitivity (Sun et al., 2007; Jeon et al., 2012). Improved glucose and lipid handling was also observed in some human trials (Brasnyo et al., 2011; Timmers et al., 2011; Bhatt et al., 2012), but not in others (Yoshino et al., 2012; Poulsen et al., 2013; van der Made et al., 2015; Timmers et al., 2016; Kjaer et al., 2017; de Ligt et al., 2018). The inconsistency in these results may originate from differences in dosing, type of resveratrol used and phenotypical differences in the target population. Administration of $150 \mathrm{mg}$ trans-resveratrol daily for 30 days was performed in three populations; middle aged normoglycemic obese individuals (Timmers et al., 2011), middle-aged overweight first-degree relatives of patients with T2DM with compromised glucose homeostasis (de Ligt et al., 2018), and 


\section{Chapter 5}

patients with T2DM (Timmers et al., 2016). In these studies, resveratrol administration consistently improved mitochondrial function while the effects on markers of insulin sensitivity were less consistent. Interestingly, the improvement in mitochondrial function was paralleled by increased muscle lipid and reduced liver lipid content in two of these studies (Timmers et al., 2011; Timmers et al., 2016), effects similar to what has been observed after exercise training.

In highly insulin-sensitive trained athletes, IMCL is stored predominantly in type I muscle fibres and dispersed alongside myofibrils (intermyofibrillar (IMF) LDs) in numerous small LDs (Daemen et al., 2018), with significantly more PLIN5 present in athletes than in T2DM individuals, a LD phenotype we referred to as an 'athlete like phenotype' (Gemmink et al., 2020). PLIN5 is a LD coat protein known to promote mitochondrial biogenesis and function (Bosma et al., 2013), in a SIRT1 and PGC1 $\alpha$ dependent fashion (Najt et al., 2020). Upon exercise training, the morphological LD characteristics in patients with T2DM changed towards an athlete-like phenotype, along with improved insulin sensitivity (Daemen et al., 2018). These observations prompted us to examine the hypothesis that in metabolically compromised individuals, a resveratrol-mediated increase in IMCL content changes the LD phenotype from the phenotype observed in metabolically compromised individuals towards the phenotype we observed in (highly insulin-sensitive) trained individuals.

To examine if the potency of resveratrol to promote muscle lipid storage is a cell autonomous effect, we first examined the effect of resveratrol in an in vitro system of cultured primary muscle cells. Subsequently, we examined if resveratrol-induced elevation of IMCL after 30 days of in vivo resveratrol supplementation changed the morphological characteristics of myocellular LDs such that they mimic the profile of lipid storage previously observed in athletes. 


\section{Methods}

\section{Ethical approval}

The current study relies on a retrospective analyses of samples obtained from three different clinical trials registered as NCT00998504, NCT01638780, and NCT02129595 (Timmers et al., 2011; Timmers et al., 2016; de Ligt et al., 2018) as well as on primary myotubes grown from previously obtained muscle biopsies (Vosselman et al., 2015). The Medical Ethic Committee of Maastricht University approved the original studies in accordance with the Declaration of Helsinki. All participants provided written informed consent before participation.

\section{Design of the in vivo study}

During the in vivo part of the study, individuals received $150 \mathrm{mg} /$ day trans-resveratrol (99.9\%) and a placebo for 30 days in a double-blinded, crossover trial with a washout period of at least 30 days. Middle-aged, obese, normoglycemic individuals (OB n=11) (Timmers et al., 2011), individuals with an increased risk of developing diabetes for having at least one first-degree relative with T2DM along with impaired glucose homeostasis (FDR n=13) (de Ligt et al., 2018) or patients with type 2 diabetes (T2DM $\mathrm{n}=17$ ) (Timmers et al., 2016) were studied. All participants were men. Details of these studies can be found in the respective papers. At baseline, body mass, BMI and body fat (DXA) were determined. After 27-30 days of supplementation (placebo or resveratrol), fasting blood plasma values of glucose and insulin (to compute HOMA-IR (Matthews et al., 1985)), free fatty acids (FFA) and triglycerides (TG) and plasma values of resveratrol and its direct metabolite dihydroresveratrol (DHR) were measured. A graded exercise test on a stationary bike was performed to assess maximal oxygen uptake $\left(\mathrm{VO}_{2} \mathrm{max}\right)$. Basal fatty acid oxidation was determined from indirect calorimetry. Ex vivo mitochondrial function was measured in permeabilized muscle fibres with high-resolution respirometry (OROBOROS Instruments, Innsbruck, Austria) by measuring state 3 respiration (ADP stimulated respiration after addition of malate and octanoylcarnitine) and state $U$ (maximal uncoupled 


\section{Chapter 5}

respiration induced by titration of FCCP).

\section{Muscle biopsies and subgroup selection}

Muscle biopsies were obtained from the $\mathrm{m}$. vastus lateralis in the morning at day 30 according to the Bergstrom method (Bergstrom, 1975) and processed for further analysis. Quantitative histological examination of IMCL by Oil red O was performed in $88 \%$ (36 out of 41 ) of the individuals. In the remaining $12 \%$, the histological appearance of the biopsy was insufficient to permit valid quantitative IMCL analysis. IMCL content (lipid area within a muscle fibre/area of the muscle fibre ${ }^{*} 100 \%$ ) along with muscle fibre typing (immunolabelling of myosin heavy chain type I) was quantified essentially according to Koopman et al. (Koopman et al., 2001). Sections from both conditions (placebo and resveratrol) were randomly mounted on the same glass slides, imaged and analysed in a blinded fashion. For all three studies, putative 'batch-effects' in the histological staining were accounted for by adjusting the data of the individual studies to the mean of that study. This resulted in a dataset with 36 individuals, showing a significant increase in IMCL content of 33\% upon resveratrol administration $(0.86 \pm 0.09 \mathrm{AU}$ in placebo vs $1.14 \pm 0.11 \mathrm{AU}$ upon resveratrol, $\mathrm{p}=0.003$, Table 1).

Given the extremely laborious nature of the detailed analysis of LD morphology, subcellular distribution and coating with relevant proteins, we studied our hypothesis in a subset of the original study population. Since the complete group consists of OB, FDR and T2DM individuals, we selected 3-4 individuals from each study that displayed an increase in IMCL (primary inclusion criteria). Secondary inclusion factors were high-quality histology (essential for valid confocal microscopy) and a match for age, $\mathrm{BMI}$ and $\mathrm{VO}_{2} \max$ with the complete group. This resulted in 10 individuals ( $3 \mathrm{OB}, 3$ FDR and 4 T2DM) whose biopsies were processed for detailed analysis of $\mathrm{LD}$ characteristics in the present study. The subgroup is a valid reflection of the complete group as indicated by the similar subject characteristics (Table 1). 
Tabel 1: Subject characteristics of the complete group and subgroup for immunohistochemistry.

\begin{tabular}{lccccc}
\hline & \multicolumn{2}{c}{ All participants (n=36) } & \multicolumn{2}{c}{ Subgroup (n=10) } & \\
& Placebo & Resveratrol & Placebo & Resveratrol & p-value ${ }^{1}$ \\
\hline Age $(\mathrm{y})$ & $61 \pm 8$ & & $58 \pm 8$ & & 0.415 \\
BMI $\left(\mathrm{kg} / \mathrm{m}^{2}\right)$ & $30.2 \pm 2.6$ & & $30.5 \pm 2.8$ & & 0.810 \\
Body fat (\%) & $26.7 \pm 3.6$ & & $27.2 \pm 3.0$ & & 0.685 \\
Resveratrol (ng/ml) & $\mathrm{ND}$ & $356.0 \pm 201.2$ & $\mathrm{ND}$ & $337.4 \pm 195.5$ & \\
DHR (ng/ml) & $\mathrm{ND}$ & $415.0 \pm 359.7$ & $\mathrm{ND}$ & $323.3 \pm 281.5$ & \\
Glucose (mmol/L) & $6.3 \pm 1.3$ & $6.3 \pm 1.4$ & $6.2 \pm 0.9$ & $6.2 \pm 1.0$ & 0.847 \\
Insulin (pmol/L) & $82.7 \pm 35.5$ & $82.2 \pm 35.0$ & $94.1 \pm 56.6$ & $92.3 \pm 47.1$ & 0.458 \\
HOMA-IR & $3.3 \pm 1.3$ & $3.3 \pm 1.6$ & $3.6 \pm 1.9$ & $3.6 \pm 1.7$ & 0.519 \\
TG (mmol/L) & $1.93 \pm 1.13$ & $1.97 \pm 0.93$ & $2.34 \pm 1.73$ & $2.38 \pm 1.29$ & 0.369 \\
FFA (mmol/L) & $0.72 \pm 0.27$ & $0.75 \pm 0.25$ & $0.78 \pm 0.23$ & $0.87 \pm 0.23$ & 0.528 \\
IMCL (AU) & $0.86 \pm 0.54$ & $1.14 \pm 0.64^{*}$ & $0.73 \pm 0.34$ & $1.44 \pm 0.74^{*}$ & 0.492 \\
VO max (ml/kg/min) & $25.3 \pm 4.8$ & $25.0 \pm 5.3$ & $26.8 \pm 4.3$ & $27.1 \pm 5.5$ & 0.375 \\
FAO (umol/kg BW/min) & $3.3 \pm 0.8$ & $3.2 \pm 0.9$ & $3.1 \pm 0.9$ & $3.0 \pm 0.9$ & 0.640 \\
State 3 (pmol/(s*mg)) & $27.9 \pm 5.9$ & $31.5 \pm 7.1^{*}$ & $29.2 \pm 6.9$ & $34.0 \pm 8.7^{*}$ & 0.561 \\
State U (pmol/(s*mg)) & $84.2 \pm 19.3$ & $93.3 \pm 20.1^{*}$ & $83.7 \pm 18.8$ & $100.4 \pm 16.6^{*}$ & 0.944 \\
\hline BM & & & & & \\
\hline
\end{tabular}

BMI, body mass index; DHR, dihydroresveratrol; FAO, fatty acid oxidation; FFA, free fatty acids; HOMA-IR, Homeostatic Model Assessment for Insulin Resistance; IMCL, intramyocellular lipids; ND, non-detectable; State 3, ADP stimulated mitochondrial respiration after addition of malate and octanoylcarnitine; State $U$, maximal uncoupled respiration induced by titration of FCCP; TG, triacylglycerol; $\mathrm{VO}_{2}$ max, maximal aerobic capacity. Data are expressed as mean $\pm S D$. ${ }^{*} p<0.05$; values are significant different between resveratrol and placebo (Student's paired t-test). ${ }^{1} p$-values reflect the difference between all participants $(n=36)$ versus the subgroup $(n=10)$ in the placebo arm (Student's unpaired $t$-test).

\section{Cell culturing}

Satellite cells were previously isolated, as described by Sparks et al. (Sparks et al., 2011), from muscle biopsies of three untrained individuals (Vosselman et al., 2015). Primary human myoblasts were cultured in growth medium containing low glucose (5.5 mM) Dulbecco's Modified Eagle Medium (DMEM) (Gibco, Thermo Fisher Scientific, Landsmeer, the Netherlands), 10\% foetal bovine serum (Gibco), 1\% fetuin (Sigma-Aldrich, Zwijndrecht, the Netherlands), 0.7\% bovine serum albumin (Sigma-Aldrich), 0.1\% dexamethasone (Sigma-Aldrich), 0.1\% Gentamycin (Gibco), $0.1 \%$ human epidermal growth factor (Gibco) and 0.02\% Fungizone (Gibco). At $70-80 \%$ confluence, medium was replaced by a differentiation medium containing 


\section{Chapter 5}

low glucose DMEM, 2\% horse serum (Gibco), 1\% fetuin and $2 \%$ penicillin and streptomycin (Sigma-Aldrich). Cells were maintained at $37^{\circ} \mathrm{C}$ and $5 \% \mathrm{CO}_{2}$ and medium was replaced every 2-3 days. After 6 days of differentiation, myotubes were incubated with or without $50 \mathrm{ng} / \mathrm{ml}$ resveratrol (R-5010, Sigma-Aldrich) for 48 hours, inspired by work from others (Kim et al., 2013).

\section{Live-cell imaging}

To monitor and quantify the effects of resveratrol incubation on IMCL accumulation in vitro over time we first performed live-cell imaging in myotubes from an untrained donor for 48 hours with 1-hour intervals. Live-cell imaging was performed using a FEI Corrsight spinning disk confocal microscope, equipped with an ORCA-Flash 4.0 V2 CMOS camera, using a 40x 0.9 N.A. air objective (Zeiss, Breda, the Netherlands) at $37^{\circ} \mathrm{C}$. Bodipy 493/503 (D3922; Molecular probes, Fisher Scientific; 1:250) was added to the medium (and remained present throughout the imaging period) to visualize the LDs and CellMask (C10046; Invitrogen, Fisher Scientific; 1:1000) was used to visualize the plasma membranes. Upon thresholding the Bodipy-derived signal, total lipid area, total LD number and average LD size were quantified in multiple wells.

\section{Visualisation of fixed cells}

Based upon the results from live-cell imaging, we picked the 48-hour time point for detailed examination in fixed cells. Human primary myotubes from three different donors were grown on \#1 coverslips and incubated with resveratrol for 48 hours. Myotubes were fixed with $3.7 \%$ formaldehyde and stained with $286 \mathrm{nM}$ DAPI (4',6-diamidino-2-phenylindole, dihydrochloride; D1306; Invitrogen) to visualize the nuclei and stained with Bodipy 493/503 (1:100) to visualize the LDs. Coverslips were mounted on glass slides with Mowiol. Imaging was performed with a FEI Corrsight spinning disk confocal microscope, using a 63x 1.4 N.A. oil immersion objective (Zeiss) and with a Nikon E800 fluorescence microscope (Nikon, Amsterdam, the Netherlands), coupled to a Nikon DS-Fi1c colour CCD camera (Nikon), using a 40x objective. Images obtained from the Corrsight were analysed similar to the live-cell 
images. Images from the Nikon microscope were analysed upon thresholding and Bodipy-derived signal was corrected for the number of nuclei.

\section{High-resolution confocal imaging of lipid droplet characteristics}

\section{Immunohistochemistry}

Biopsies from the $\mathrm{m}$. vastus lateralis were frozen in liquid nitrogen-cooled isopentane. Five $\mu \mathrm{m}$ thick sections were serial cut with a cryostat (CM3050; Leica Biosystems, Amsterdam, the Netherlands) at $-20^{\circ} \mathrm{C}$, mounted on glass slides and air-dried for 30 minutes. To ensure identical incubation conditions, sections from placebo and resveratrol were mounted on the same glass slide. Slides were fixed with $3.7 \%$ formaldehyde and permeabilized with $0.25 \%$ TX-100. Primary antibodies against Laminin (L9393; Sigma-Aldrich; 1:25), MHC-1 (A4.840; Developmental Studies Hybridoma Bank, Iowa, USA; 1:25) and PLIN5 (GP31; ProGen Biotechnik, Heidelberg, Germany; 1:25) were used. Appropriate secondary antibodies were conjugated with AlexaFluor (AF) 405, AF 555 and AF 647. Bodipy 493/503 (1:100) was applied simultaneously with the secondary antibodies to visualize LDs.

\section{Imaging and quantification}

Imaging and quantification of IMCL, LD size, number and subcellular distribution in type I and type II muscle fibres was essentially performed according to Daemen et al. (Daemen et al., 2018). Briefly, cross-sections of circa 20 muscle fibres (with a fibre type I/II ratio that matched the individual fibre typing (on average $45 \%$ type I and $55 \%$ type II fibres)) were imaged with a Leica TCS SP8 confocal microscope using a 63x 1.4 N.A. oil immersion objective. Deconvolution was performed with Huygens Professional software (Scientific Volume Imaging B.V., Hilversum, the Netherlands), quantification by ImageJ version 1.52p (NIH, Bethesda, USA) (Schneider et al., 2012) and further calculations with MATLAB R2013a (The Mathworks, Inc., Natick, Massachusetts, USA). IMCL was determined as the total lipid area corrected for the total cell area. LD number is defined as total LD count corrected for cell area and 


\section{Chapter 5}

LD size is the total lipid area divided by the total LD count. Subcellular distribution was analysed by making a distinction between intermyofibrillar (IMF) LDs or subsarcolemmal (SS) LDs, whereas the SS region represents the outer $8 \%$ of each cell and the IMF region the remaining $92 \%$ of the cell. Moreover, LDs were subdivided into PLIN5-positive LDs (PLIN5+) and LDs without PLIN5 (PLIN5-), as previously described (Gemmink et al., 2018).

\section{Western blot}

Protein levels of PLIN5 in human muscle tissue were determined by Western blot in 8/10 individuals from the subgroup. Tissues were lysed using Bio-Plex Cell Lysis kit (171-304011; Bio-Rad, Veenendaal, the Netherlands) and equal amounts of protein were loaded onto the gels (Figure S1). After gel electrophoresis, proteins were transferred by Western blotting and a Revert total protein stain (LI-COR Bioscience, Westburg, Leusden, the Netherlands) was performed to determine the protein quality and the protein quantity on the blots. Then, the membranes were blocked with LI-COR Blocking buffer for $30 \mathrm{~min}$ and incubated with a primary antibody against PLIN5 (1:2500, diluted in LI-COR Blocking buffer) overnight at room temperature. A IRDye800-conjugated secondary antibody was used for visualization PLIN5 by an Odyssey near infrared scanner (LI-COR Biosciences). Western blots were quantified with Image Studio version 5.2 (LI-COR Biosciences) and values were normalized to total protein stain.

\section{Statistical analysis}

Statistical analysis was performed with Prism version 8.2 (GraphPad, San Diego, CA, USA) and SPSS, version 25 (IBM Corp., Armonk, NY, USA). After testing for normality (Shapiro-Wilk normality test), differences between placebo and resveratrol were tested by two-tailed Student's paired t-test. Time course analysis was performed by two-way ANOVA. Results are presented as mean \pm SEM, unless indicated otherwise. A p-value $<0.05$ was considered statistically significant. 


\section{Results}

\section{Resveratrol stimulates lipid accumulation in a cell autonomous manner}

Resveratrol has previously been shown to augment IMCL in vivo (Table 1) (Timmers et al., 2011; Timmers et al., 2016; de Ligt et al., 2018). To examine if these effects were muscle cell autonomous, we incubated human primary muscle cells with resveratrol and used fluorescent microscopy to determine intracellular lipid accumulation. First, lipid accumulation was followed by live-cell imaging after resveratrol incubation, revealing an overall significant time and treatment effect. Within the first 6 hours, LD area was $12 \%$ higher after resveratrol treatment compared to control. This difference between the conditions was sustained and increased towards $\sim 41-46 \%$ from 31 to 48 hours (Figure 1A). This increase in LD area originated from an increase in LD number (Figure 1B), with a 15\% greater LD number within the first 6 hours upon resveratrol treatment, which further increased to $46-49 \%$ between 31 and 48 hours compared to control. No changes in LD size were observed (Figure 1C). Subsequently, we chose the 48 hour time point to perform cell experiments with myotubes from three different untrained donors that were incubated with resveratrol. In fixed cells, lipid area increased after 48 hours (1.7-fold increase, Figure 1D). This increase originates predominantly from an increase in LD number (placebo $323 \pm 265$ vs resveratrol 917 \pm 445 , Figure 1E) rather than size (placebo $0.39 \pm 0.01 \mu \mathrm{m}^{2}$ vs resveratrol $0.30 \pm 0.03$ $\mu \mathrm{m}^{2}$, Figure 1F). Moreover, LD accumulation was related to the number of nuclei to obtain values related to cell number and promote comparison to the in vivo analyses. From multiple experiments, we observed a 2.3 -fold increase $(\mathrm{p}=0.038$, Figure 1G) in lipid area relative to nuclei (placebo vs resveratrol, Figure $\mathbf{1 H}$ vs $\mathbf{1 I}$ ). 

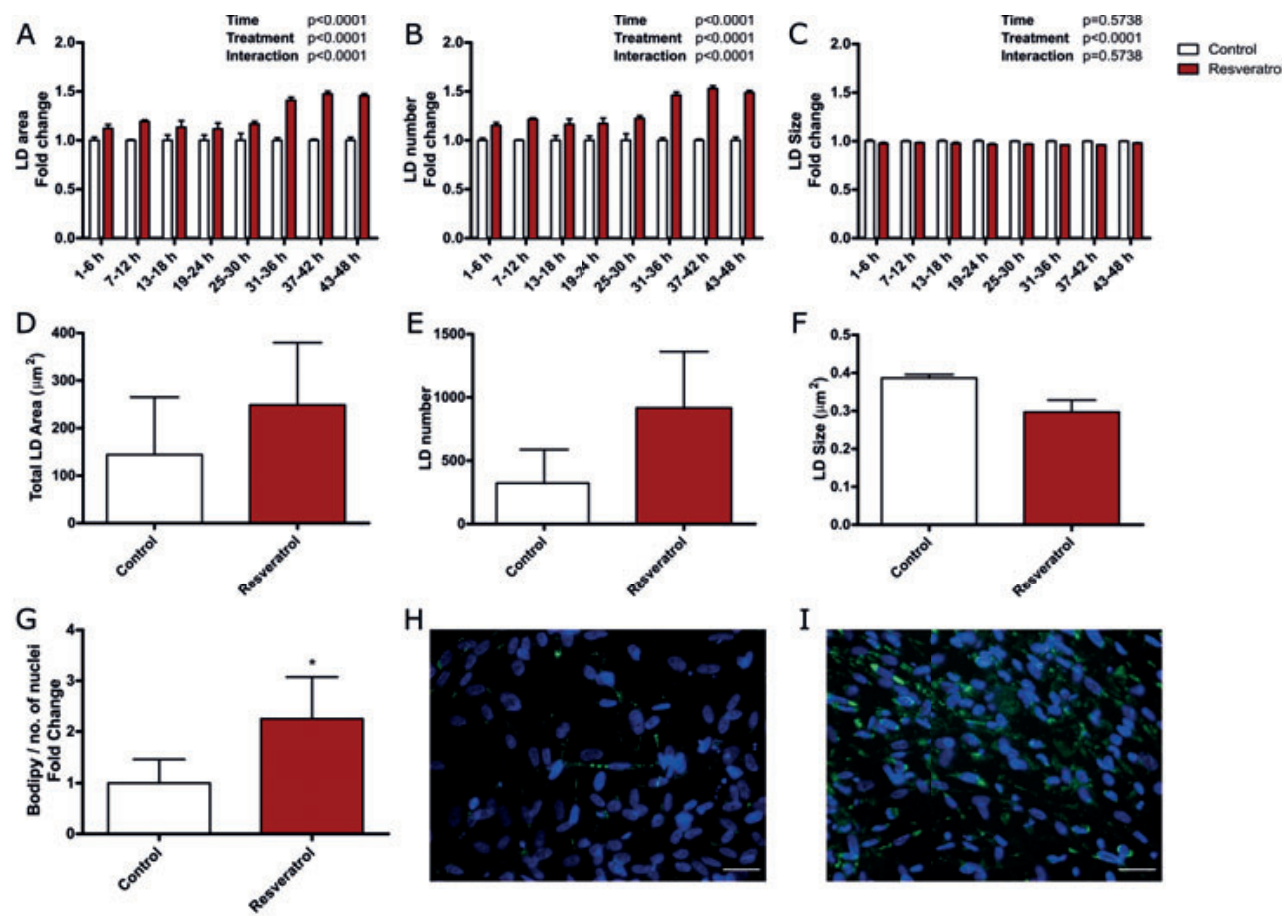

Figure 1. Resveratrol treatment stimulates lipid accumulation in human muscle cells. Human myoblasts were cultured and differentiated and incubated with or without $50 \mathrm{ng} / \mathrm{ml}$ resveratrol. A-C) Time course analysis for $L D$ area $(A), L D$ number $(B)$ and $L D$ size $(C)$. Data are obtained from 1 donor, from multiple wells. Data are presented as mean fold change \pm SEM ( $n=6$ time points); analysed by two-way ANOVA. D-F) Effect of resveratrol on total $L D$ area $(D)$, total $L D$ number $(E)$ and average $L D$ size $(F)$ after 48 hours incubation. Data are obtained from 3 donors, from multiple wells in fixed cells. Data are presented as mean \pm SEM (n=3). G-I) After an incubation of 48 hours, cells were fixed and nuclei were stained with DAPI (blue) and neutral lipids with Bodipy 493/503 (green). Bodipy area/nuclei was determined with or without resveratrol. Data are obtained from three donors and 2-4 repeated experiments. Data are presented as mean fold change \pm SEM $(n=9)$. * significantly different from control $(p<0.05$, paired t-test). Images are representative for $G$ incubated without $(H)$ and with resveratrol $(I)$. Scale bar $=40 \mu \mathrm{m}$.

\section{Resveratrol supplementation in vivo induces LD accumulation in a fibre type and subcellular region-specific fashion.}

In metabolically compromised individuals, 30 days of resveratrol supplementation augmented IMCL content (Table 1). Detailed analysis revealed that IMCL increased in type I muscle fibres (placebo $0.97 \pm 0.16 \%$ vs resveratrol $1.26 \pm 0.09 \%$; $=0.030$; Figure 2A+D) but not in type II muscle fibres (placebo $0.62 \pm 0.09 \%$ vs resveratrol 0.66 $\pm 0.15 \%$; $=0.794$; Figure $2 \mathrm{~A}+\mathrm{E})$. While in the cell model, resveratrol-induced increase in LD originated predominantly from an increase in LD number, the significant increase in total IMCL in vivo originates from the combined effect of a non-significant 

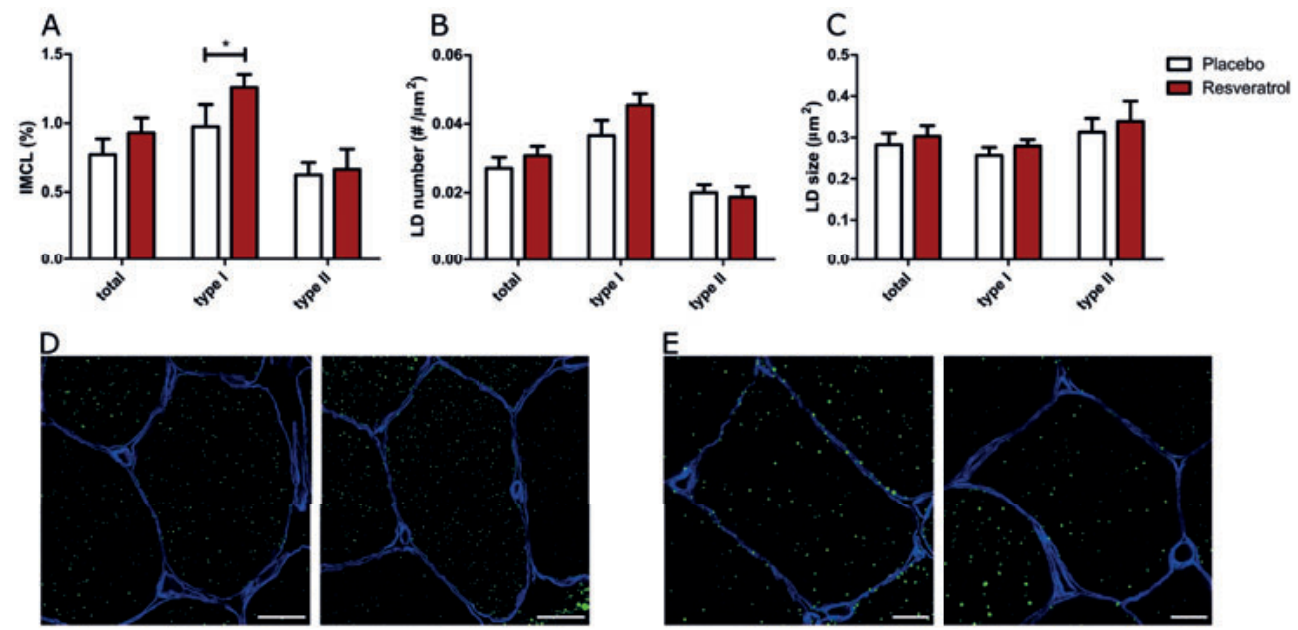

Figure 2. Resveratrol increases IMCL in type I muscle fibres. Effect of in vivo resveratrol supplementation of $150 \mathrm{mg} /$ day trans-resveratrol on IMCL (A), LD number $(\boldsymbol{B})$ and LD size (C) in human muscle biopsies. IMCL was determined by lipid area (Bodipy staining)/cell area*100\%. Parameters were analysed in type I muscle fibres, type II muscle fibres and for both fibre types combined. Data are presented as mean \pm SEM (n=10); ${ }^{*} p<0.05$ (paired t-test). Images are representative for IMCL in type I muscle fibres (D) and type II (E) muscle fibres with placebo on the left and resveratrol on the right. Cell membranes are stained in blue and LDs in green. Scale bar is $20 \mu \mathrm{m}(D)$ or $10 \mu \mathrm{m}$ (E).

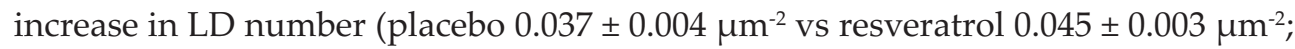
$\mathrm{p}=0.083$; Figure 2B) and a non-significant increase in size (placebo $0.26 \pm 0.02 \mu^{2} \mathrm{vs}$ resveratrol $0.28 \pm 0.02 \mu \mathrm{m}^{2} ; \mathrm{p}=0.181$; Figure $2 \mathrm{C}$ ).

Examination of subcellular distribution of LDs in type I fibres revealed an increase in IMCL in the SS as well as in the IMF region $(2.04 \pm 0.33 \%$ vs $2.83 \pm 0.23 \%$, p =0.016 for placebo vs resveratrol in the SS region and $0.90 \pm 0.14 \%$ vs $1.15 \pm 0.08 \%$, p $=0.026$ for placebo vs resveratrol in the IMF region; Figure 3A). While in the SS region, the increase in total LD content was due to a non-significant increase in LD number (placebo $0.077 \pm 0.010 \mu \mathrm{m}^{-2}$ vs resveratrol $0.100 \pm 0.008 \mu \mathrm{m}^{-2} ; \mathrm{p}=0.069$, Figure 3B) and LD size (placebo $0.26 \pm 0.02 \mu \mathrm{m}^{2}$ vs resveratrol $0.29 \pm 0.02 \mu \mathrm{m}^{2} ; \mathrm{p}=0.080$, Figure $3 \mathrm{C}$ ), the change in LD content in the IMF region originated from a non-significant increase

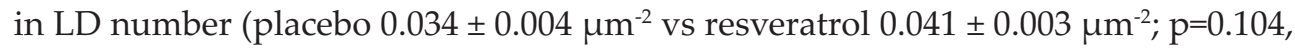
Figure 3B) and a significant increase in LD size (placebo $0.25 \pm 0.02 \mu^{2}$ vs resveratrol $0.28 \pm 0.02 \mu \mathrm{m}^{2} ; \mathrm{p}=0.042$, Figure $3 \mathrm{C}$ ). No significant changes were observed in type II muscle fibres for IMCL (SS: $p=0.692 ; \mathrm{IMF}: \mathrm{p}=0.881$ ), LD number (SS: $\mathrm{p}=0.757$; IMF: $\mathrm{p}=0.813$ ) and LD size (SS: $\mathrm{p}=0.262$; IMF: $\mathrm{p}=0.452$ ) (Figure 3A-C). 

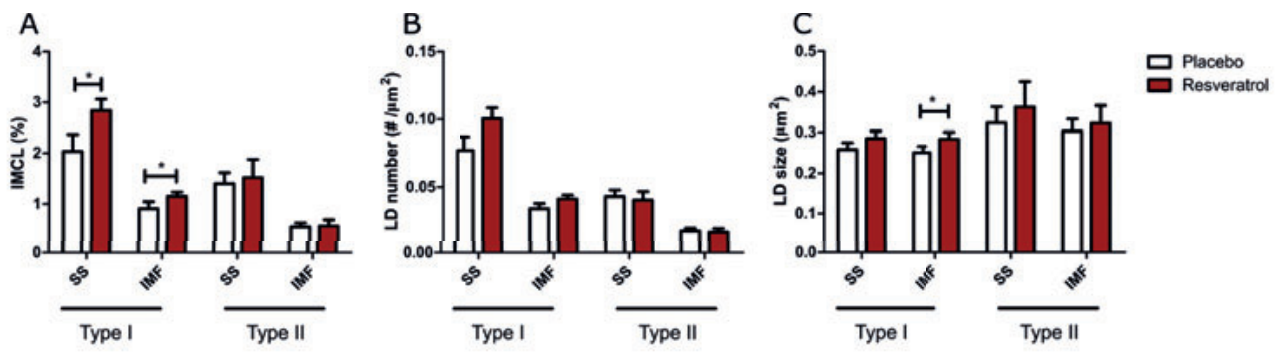

Figure 3. Resveratrol increases IMCL in type I muscle fibres in both the intermyofibrillar (IMF) and subsarcolemmal (SS) region. Effect of in vivo resveratrol supplementation of $150 \mathrm{mg} /$ day transresveratrol on IMCL $(\boldsymbol{A})$, lipid droplet $(L D)$ number $(\boldsymbol{B})$ and $L D$ size $(\boldsymbol{C})$ in human muscle biopsies. $L D$ s are analysed separately in the IMF region and SS region, in both type I and type II muscle fibres. IMCL was determined by lipid area (Bodipy staining)/cell area*100\%. Data are presented as mean \pm SEM $(n=10)$; ${ }^{*} p<0.05$ (paired $t$-test).

\section{Resveratrol promotes IMCL storage predominantly in LDs coated with PLIN5 present in type I muscle fibres}

We and others previously observed that preferential storage of lipids in LDs coated with PLIN5 permits augmented IMCL content in oxidative muscle, while not impeding insulin sensitivity (Gemmink et al., 2016; Shepherd et al., 2017; Gemmink et al., 2018). To examine the preferred lipid storage in LDs coated with PLIN5 in the resveratrol-mediated increase in lipid content in oxidative muscle, we made the distinction between LDs coated with PLIN5 (PLIN5+ LDs) and LDs without PLIN5 (PLIN5- LDs) (Figure 4E). Interestingly, we observed a significant increase in lipid storage in PLIN5+ LDs (delta increase 0.231 percent point, $\mathrm{p}=0.024$, Figure $4 \mathrm{~A}+\mathrm{D}$ ) but not in PLIN5- LDs (delta increase 0.067 percent point, $p=0.457$, Figure $4 A+D$ ) in type I muscle fibres upon resveratrol supplementation. The increase in lipid storage in PLIN5+ LDs was accounted for by a non-significant increased number of PLIN5+ LDs $(\mathrm{p}=0.091$, Figure 4B) and a significant increase in size $(\mathrm{p}=0.023$; Figure $4 \mathrm{C})$. For type II muscle fibres (known to have limited PLIN5 content) no such observations were made (IMCL type II fibres PLIN5+ LDs; delta increase -0.022 percent point, $\mathrm{p}=0.466$ ). PLIN5 protein content was not different between placebo and resveratrol (Figure 4F). 

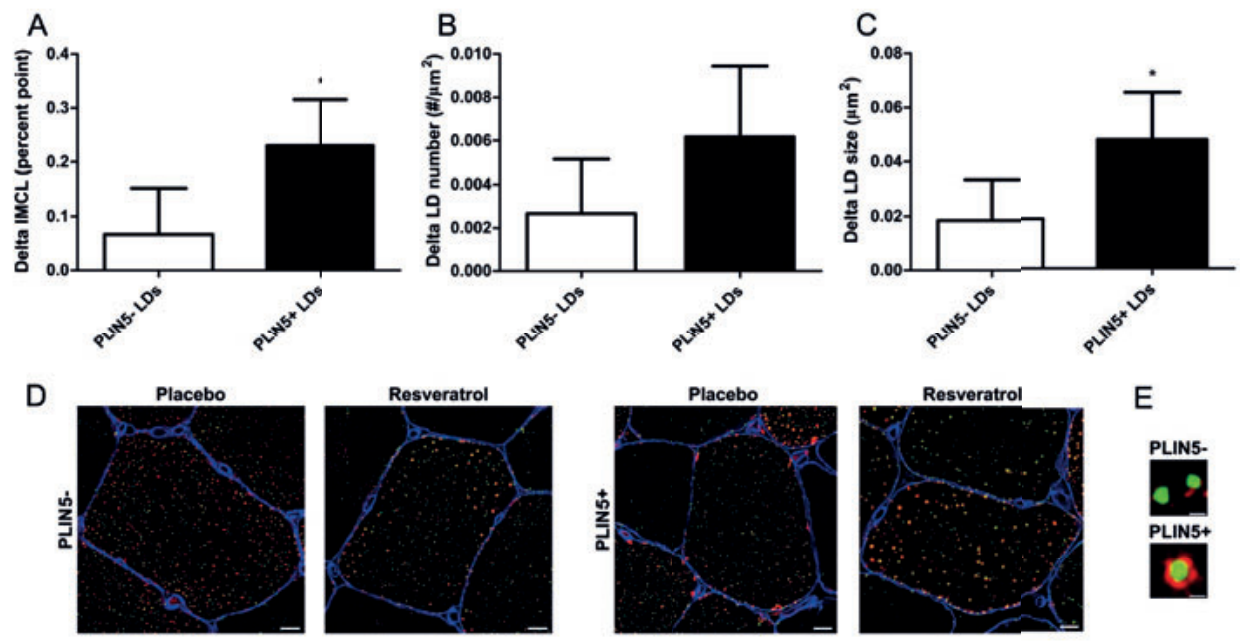

E

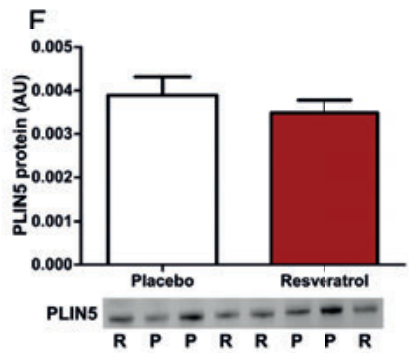

Figure 4. Resveratrol increases IMCL in specifically PLIN5 coated lipid droplets in type I muscle fibres. LDs are distinguished between PLIN5 negative LDs (PLIN5-) and PLIN5-positive LDS (PLIN5+). The increase in IMCL after supplementation of $150 \mathrm{mg} /$ day trans-resveratrol was significant in PLIN5+ LDs and not in PLIN5- LDs (A). This increase in IMCL was due to a non-significant increase in LD number (B) and to a significant increase in $L D$ size $(\boldsymbol{C})$. Data are from human muscle biopsies and presented as mean delta \pm SEM ( $n=10)$; 'significant difference between placebo and resveratrol $(p<0.05$; paired $t$-test). The increase in IMCL after resveratrol supplementation is visualized in representative images for PLIN5- LDs and PLIN5+ LDs. Scale bar $=10 \mu m$ (D). A LD is PLIN5+ when there is overlay of PLIN5 staining with the $L D$ staining. Scale bar $=1 \mu m(E)$. Total PLIN5 protein did not change after resveratrol supplementation determined via Western blot (F). Data are from human muscle biopsies and presented as mean \pm SEM $(n=8)$. Blot images are from four different individuals. $P=$ placebo; $R=$ resveratrol. 


\section{Chapter 5}

\section{Discussion}

The current study showed that resveratrol augments IMCL content in human skeletal muscle and results in remodelling of LDs in a cell autonomous fashion. In human clinical trials, it has been observed that 30 days of $150 \mathrm{mg} /$ day resveratrol supplementation can augment overall IMCL storage without impeding insulin sensitivity in metabolically compromised individuals. Hypothetically, this is indicative of a benign 'athlete-like' IMCL storage pattern. In line with this hypothesis, we observed that resveratrol particularly promoted IMCL storage in oxidative type I muscle fibres and LDs coated with PLIN5.

The cell model indicates that the IMCL inducing effects of resveratrol are not secondary to alterations in whole-body lipid metabolism and the increase in IMCL upon resveratrol originated from an increase in LD number. In the human intervention study, resveratrol also induced a significant increase in overall IMCL content. The increase in IMCL originates from a $24 \%$ non-significant $(p=0.083$ ) increase in LD number without profound changes in LD size. The direction of these morphological changes indeed matches with an athlete like phenotype (Gemmink et al., 2020). Unlike in athletes, the resveratrol-mediated increase in IMCL was not only found in intermyofibrillar region but in the subsarcolemmal region as well.

Type I muscle fibres are known to store higher amounts of lipids compared to type II muscle fibres and are more oxidative (van Loon et al., 2003; van Loon et al., 2004; Schrauwen-Hinderling et al., 2006). For storage of IMCL in type I fibres, we previously reported that the number of LDs correlates positively with insulin sensitivity, whereas in type II fibres the size of LDs correlates negatively with insulin sensitivity (Daemen et al., 2018). Thus, the preferred storage of IMCL in type I fibres rather than in type II fibres and in more LDs rather than large LDs matches with the observation that the resveratrol-mediated increase in IMCL does not impede insulin sensitivity.

Interestingly, the increase in IMCL observed in type I muscle fibres was most profound in LDs coated with PLIN5. Preferred storage of IMCL in PLIN5+ LDs was previously observed in trained athletes (Gemmink et al., 2018) and found to 
ameliorate insulin resistance (Gemmink et al., 2016). Again, this fits the hypothesis that resveratrol promotes lipid storage in a benign, athlete-like fashion. PLIN5 is a LD coat protein with a major role in lipid turnover (Wang et al., 2011a; MacPherson et al., 2013) and supports to match LD lipolysis with mitochondrial fat oxidation to prevent lipotoxicity and insulin resistance (Wang et al., 2011b; Bosma et al., 2013; Laurens et al., 2016). To our knowledge, no research has currently been performed to investigate the role of resveratrol on PLIN5 in humans. In mice, resveratrol was reported to increase PLIN5 protein content in the muscle (Mehdi et al., 2018). In the present human study, we did not find an increase in total PLIN5 content. In fact, the observed increase in PLIN5+ LDs (in the absence of changes in total PLIN5 content) is suggestive of PLIN5 redistribution (Gemmink et al., 2016; Shepherd et al., 2017). The current study design did not permit more mechanistic studies as to how resveratrol may mediate augmented lipid storage in PLIN5-coated LDs in type I fibres. While pre-clinical models revealed activation of PGC1 $\alpha$ upon resveratrol (Lagouge et al., 2006), this is not observed consistently (Higashida et al., 2013). Although the results on induction of PGC1 $\alpha$ in human skeletal muscle by resveratrol are equivocal, it is important to note that overexpression of PGC1 $\alpha$ in skeletal muscle induces gene and protein expression of a series of proteins involved in LD dynamics including PLIN5 (Koves et al., 2013).

In contrast to observations in trained athletes, the resveratrol-mediated increase in IMCL was not limited to the IMF region, but was also observed in the SS region. High levels of SS LDs are associated with lower insulin sensitivity (Nielsen et al., 2010; Chee et al., 2016; Daemen et al., 2018). Fibre type specific examinations revealed that IMCL stored in SS LDs impeded insulin sensitivity particularly if found in type II and not type I fibres (Daemen et al., 2018). The induction of IMCL in SS LDs upon resveratrol was almost exclusively observed in type I fibres, possibly explaining why this increase was not paralleled by compromised insulin sensitivity. Why resveratrol promotes lipid storage in both the SS and IMF compartments is currently not known. Based on anatomical location, one could speculate that the vicinity of SS LDs with the capillary network encompassing the type I fibres renders SS LDs, a physically 


\section{Chapter 5}

nearby storage site for extracted circulatory fats. On the other hand, IMF LDs are closely located to the contractile elements (Tarnopolsky et al., 2007) and have been suggested to fuel mitochondrial fat oxidation during prolonged submaximal exercise (Hood, 2001). Upon exercise or exercise training, fatty acids can be released from SS LD's and be re-incorporated in IMF LDs to fuel mitochondrial oxidation upon demand. The observation that lipid content in SS LDs decreases after exercise without changes in lipid content in IMF LDs supports this notion (Nielsen et al., 2010; Li et al., 2014). Regular physical exercise is an obvious difference between changes in the athletes versus the resveratrol-mediated increase in IMCL content in a metabolically compromised non-physically active population observed here. Regular physical exercise requires an efficient system for IMF LD turnover. Administration of resveratrol in the absence of regular exercise may not trigger such a mechanism, which may explain the increase in both IMF and SS LDs.

This is the first study that has examined the effect of resveratrol on intramyocellular lipid storage in primary human muscle cells as well as in vivo in humans. Our unique quantitative microscopy approach permits examination of the effect of resveratrol in a fibre type and region-specific fashion at the level of individual lipid droplets. More conventional approaches will only give results based on muscle homogenates or extractions that will not result in the type of detailed information needed to explain how resveratrol can augment IMCL content while not impeding insulin sensitivity. The downside of this approach is that it is extremely laborious, which limits the number of subjects that could be included in the current study.

In conclusion, we observed that resveratrol stimulates lipid accumulation in human muscle cells in culture as well as in vivo in metabolically compromised humans. The pattern of IMCL storage upon administration of resveratrol mimics the pattern of IMCL storage in trained athletes. Deduced from this resemblance, a phenotype of benign IMCL storage is emerging with LDs being stored in type I muscle fibres in numerous small LDs most of which are coated with PLIN5. Thus, a favourable remodelling of the IMCL storage pattern can be achieved upon resveratrol administration. 


\section{Supplementary data for chapter 5}

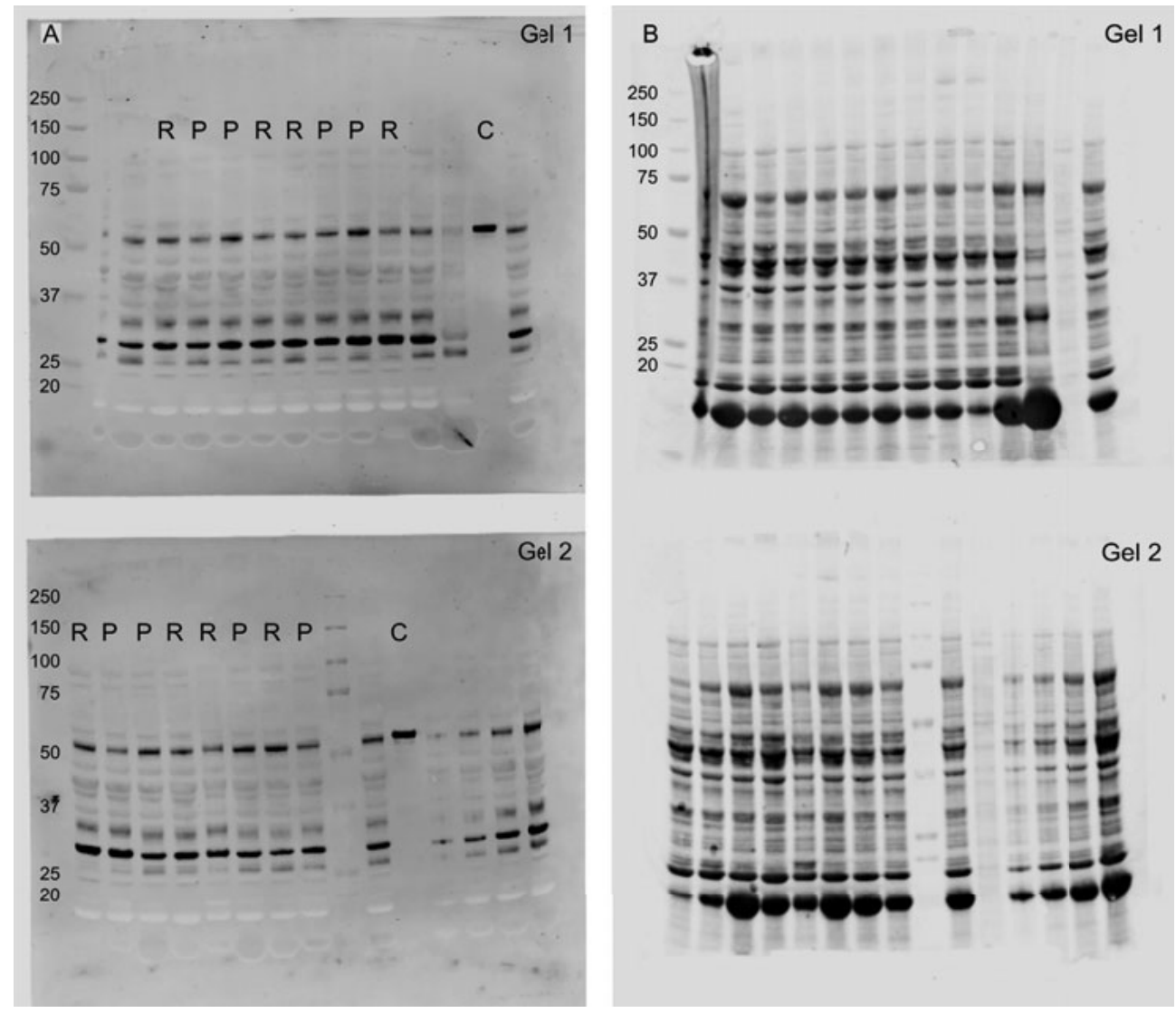

Figure S1. PLIN5 western blot. Images of the PLIN5 western blot (A) and Revert total protein stain $(\boldsymbol{B})$. In eight individuals, PLIN5 protein was analysed after placebo $(P)$ and resveratrol $(R)$ supplementation, where 4 paired samples were run on each gel. Samples were compared with a positive control $(C)$. The numbers of the protein ladders are in $k D$ and present in lane 1 on gel 1 and in lane 9 on gel 2. 


\section{References}

Alberdi G, Rodriguez VM, Macarulla MT, Miranda J, Churruca I \& Portillo MP. (2013). Hepatic lipid metabolic pathways modified by resveratrol in rats fed an obesogenic diet. Nutrition 29, 562-567.

Bergstrom J. (1975). Percutaneous needle biopsy of skeletal muscle in physiological and clinical research. Scand J Clin Lab Invest 35, 609-616.

Bhatt JK, Thomas S \& Nanjan MJ. (2012). Resveratrol supplementation improves glycemic control in type 2 diabetes mellitus. Nutr Res 32, 537-541.

Bosma M, Sparks LM, Hooiveld GJ, Jorgensen JA, Houten SM, Schrauwen P, Kersten S \& Hesselink MKC. (2013). Overexpression of PLIN5 in skeletal muscle promotes oxidative gene expression and intramyocellular lipid content without compromising insulin sensitivity. Bba-Mol Cell Biol L 1831, 844-852.

Brasnyo P, Molnar GA, Mohas M, Marko L, Laczy B, Cseh J, Mikolas E, Szijarto IA, Merei A, Halmai R, Meszaros LG, Sumegi B \& Wittmann I. (2011). Resveratrol improves insulin sensitivity, reduces oxidative stress and activates the Akt pathway in type 2 diabetic patients. Br J Nutr 106, 383-389.

Chee C, Shannon CE, Burns A, Selby AL, Wilkinson D, Smith K, Greenhaff PL \& Stephens FB. (2016). Relative Contribution of Intramyocellular Lipid to Whole-Body Fat Oxidation Is Reduced With Age but Subsarcolemmal Lipid Accumulation and Insulin Resistance Are Only Associated With Overweight Individuals. Diabetes 65, 840-850.

Chen LL, Zhang HH, Zheng J, Hu X, Kong W, Hu D, Wang SX \& Zhang P. (2011). Resveratrol attenuates high-fat diet-induced insulin resistance by influencing skeletal muscle lipid transport and subsarcolemmal mitochondrial beta-oxidation. Metabolism 60, 1598-1609.

Daemen S, Gemmink A, Brouwers B, Meex RCR, Huntjens PR, Schaart G, Moonen-Komips E, Jorgensen J, Hoeks J, Schrauwen P \& Hesselink MKC. (2018). Distinct lipid droplet characteristics and distribution unmask the apparent contradiction of the athlete's paradox. Molecular Metabolism 17, 71-81.

de Ligt M, Bruls YMH, Hansen J, Habets MF, Havekes B, Nascimento EBM, Moonen-Kornips E, Schaart G, Schrauwen-Hinderling VB, van Marken Lichtenbelt W \& Schrauwen P. (2018). Resveratrol improves ex vivo mitochondrial function but does not affect insulin sensitivity or brown adipose tissue in first degree relatives of patients with type 2 diabetes. Mol Metab 12, 39-47.

DeFronzo RA \& Tripathy D. (2009). Skeletal muscle insulin resistance is the primary defect in type 2 diabetes. Diabetes Care 32 Suppl 2, S157-163.

Gemmink A, Bosma M, Kuijpers HJ, Hoeks J, Schaart G, van Zandvoort MA, Schrauwen P \& Hesselink MK. (2016). Decoration of intramyocellular lipid droplets with PLIN5 modulates fasting-induced insulin resistance and lipotoxicity in humans. Diabetologia 59, 1040-1048.

Gemmink A, Daemen S, Brouwers B, Huntjens PR, Schaart G, Moonen-Kornips E, Jorgensen J, Hoeks J, Schrauwen P \& Hesselink MKC. (2018). Dissociation of intramyocellular lipid storage and insulin resistance in trained athletes and type 2 diabetes patients; involvement of perilipin 5? J Physiol 596, 857-868.

Gemmink A, Schrauwen P \& Hesselink MKC. (2020). Exercising your fat (metabolism) into shape: a muscle-centred view. Diabetologia 63, 1453-1463. 
Gong L, Guo S \& Zou Z. (2020). Resveratrol ameliorates metabolic disorders and insulin resistance in high-fat diet-fed mice. Life Sci 242, 117212.

Goodpaster BH, He J, Watkins S \& Kelley DE. (2001). Skeletal muscle lipid content and insulin resistance: evidence for a paradox in endurance-trained athletes. J Clin Endocrinol Metab 86, 5755-5761.

Goodpaster BH, Theriault R, Watkins SC \& Kelley DE. (2000). Intramuscular lipid content is increased in obesity and decreased by weight loss. Metabolism 49, 467-472.

Higashida K, Kim SH, Jung SR, Asaka M, Holloszy JO \& Han DH. (2013). Effects of resveratrol and SIRT1 on PGC-1alpha activity and mitochondrial biogenesis: a reevaluation. PLoS Biol 11, e1001603.

Hood DA. (2001). Plasticity in skeletal, cardiac, and smooth muscle - Invited review: Contractile activity-induced mitochondrial biogenesis in skeletal muscle. J Appl Physiol 90, 11371157.

Jeon BT, Jeong EA, Shin HJ, Lee Y, Lee DH, Kim HJ, Kang SS, Cho GJ, Choi WS \& Roh GS. (2012). Resveratrol attenuates obesity-associated peripheral and central inflammation and improves memory deficit in mice fed a high-fat diet. Diabetes 61, 1444-1454.

Kim MY, Lim JH, Youn HH, Hong YA, Yang KS, Park HS, Chung S, Ko SH, Shin SJ, Choi BS, Kim HW, Kim YS, Lee JH, Chang YS \& Park CW. (2013). Resveratrol prevents renal lipotoxicity and inhibits mesangial cell glucotoxicity in a manner dependent on the AMPK-SIRT1-PGC1alpha axis in db/db mice. Diabetologia 56, 204-217.

Kjaer TN, Ornstrup MJ, Poulsen MM, Stodkilde-Jorgensen H, Jessen N, Jorgensen JOL, Richelsen B \& Pedersen SB. (2017). No Beneficial Effects of Resveratrol on the Metabolic Syndrome: A Randomized Placebo-Controlled Clinical Trial. J Clin Endocr Metab 102, 1642-1651.

Koopman R, Schaart G \& Hesselink MK. (2001). Optimisation of oil red O staining permits combination with immunofluorescence and automated quantification of lipids. Histochem Cell Biol 116, 63-68.

Koves TR, Sparks LM, Kovalik JP, Mosedale M, Arumugam R, DeBalsi KL, Everingham K, Thorne L, Phielix E, Meex RC, Kien CL, Hesselink MKC, Schrauwen P \& Muoio DM. (2013). PPAR gamma coactivator-1 alpha contributes to exercise-induced regulation of intramuscular lipid droplet programming in mice and humans. J Lipid Res 54, 522-534.

Krssak M, Falk Petersen K, Dresner A, DiPietro L, Vogel SM, Rothman DL, Roden M \& Shulman GI. (1999). Intramyocellular lipid concentrations are correlated with insulin sensitivity in humans: a ${ }^{1} \mathrm{H}$ NMR spectroscopy study. Diabetologia 42, 113-116.

Lagouge M, Argmann C, Gerhart-Hines Z, Meziane H, Lerin C, Daussin F, Messadeq N, Milne J, Lambert P, Elliott P, Geny B, Laakso M, Puigserver P \& Auwerx J. (2006). Resveratrol improves mitochondrial function and protects against metabolic disease by activating SIRT1 and PGC-1alpha. Cell 127, 1109-1122.

Laurens C, Bourlier V, Mairal A, Louche K, Badin PM, Mouisel E, Montagner A, Marette A, Tremblay A, Weisnagel JS, Guillou H, Langin D, Joanisse DR \& Moro C. (2016). Perilipin 5 fine-tunes lipid oxidation to metabolic demand and protects against lipotoxicity in skeletal muscle. Sci Rep-Uk 6.

Li Y, Lee S, Langleite T, Norheim F, Pourteymour S, Jensen J, Stadheim HK, Storas TH, Davanger S, Gulseth HL, Birkeland KI, Drevon CA \& Holen T. (2014). Subsarcolemmal lipid droplet responses to a combined endurance and strength exercise intervention. 
Physiol Rep 2.

MacPherson RE, Ramos SV, Vandenboom R, Roy BD \& Peters SJ. (2013). Skeletal muscle PLIN proteins, ATGL and CGI-58, interactions at rest and following stimulated contraction. Am J Physiol Regul Integr Comp Physiol 304, R644-650.

Matthews DR, Hosker JP, Rudenski AS, Naylor BA, Treacher DF \& Turner RC. (1985). Homeostasis model assessment: insulin resistance and beta-cell function from fasting plasma glucose and insulin concentrations in man. Diabetologia 28, 412-419.

Metcalfe LK, Smith GC \& Turner N. (2019). Defining lipid mediators of insulin resistance: controversies and challenges. J Mol Endocrinol 62, R65-R82.

Middleton KR, Anton SD \& Perri MG. (2013). Long-Term Adherence to Health Behavior Change. Am J Lifestyle Med 7, 395-404.

Najt CP, Khan SA, Heden TD, Witthuhn BA, Perez M, Heier JL, Mead LE, Franklin MP, Karanja KK, Graham MJ, Mashek MT, Bernlohr DA, Parker L, Chow LS \& Mashek DG. (2020). Lipid Droplet-Derived Monounsaturated Fatty Acids Traffic via PLIN5 to Allosterically Activate SIRT1. Mol Cell 77, 810-824 e818.

Nielsen J, Christensen AE, Nellemann B \& Christensen B. (2017). Lipid droplet size and location in human skeletal muscle fibers are associated with insulin sensitivity. Am J Physiol Endocrinol Metab 313, E721-E730.

Nielsen J, Mogensen M, Vind BF, Sahlin K, Hojlund K, Schroder HD \& Ortenblad N. (2010). Increased subsarcolemmal lipids in type 2 diabetes: effect of training on localization of lipids, mitochondria, and glycogen in sedentary human skeletal muscle. Am J Physiol Endocrinol Metab 298, E706-713.

Pan DA, Lillioja S, Kriketos AD, Milner MR, Baur LA, Bogardus C, Jenkins AB \& Storlien LH. (1997). Skeletal muscle triglyceride levels are inversely related to insulin action. Diabetes 46, 983-988.

Perreault L, Newsom SA, Strauss A, Kerege A, Kahn DE, Harrison KA, Snell-Bergeon JK, Nemkov T, D’Alessandro A, Jackman MR, MacLean PS \& Bergman BC. (2018). Intracellular localization of diacylglycerols and sphingolipids influences insulin sensitivity and mitochondrial function in human skeletal muscle. JCI Insight 3.

Poulsen MM, Vestergaard PF, Clasen BF, Radko Y, Christensen LP, Stodkilde-Jorgensen H, Moller N, Jessen N, Pedersen SB \& Jorgensen JO. (2013). High-dose resveratrol supplementation in obese men: an investigator-initiated, randomized, placebocontrolled clinical trial of substrate metabolism, insulin sensitivity, and body composition. Diabetes 62, 1186-1195.

Schneider CA, Rasband WS \& Eliceiri KW. (2012). NIH Image to ImageJ: 25 years of image analysis. Nat Methods 9, 671-675.

Schrauwen-Hinderling VB, Hesselink MK, Schrauwen P \& Kooi ME. (2006). Intramyocellular lipid content in human skeletal muscle. Obesity (Silver Spring) 14, 357-367.

Shepherd SO, Strauss JA, Wang Q, Dube JJ, Goodpaster B, Mashek DG \& Chow LS. (2017). Training alters the distribution of perilipin proteins in muscle following acute free fatty acid exposure. J Physiol 595, 5587-5601.

Sparks LM, Moro C, Ukropcova B, Bajpeyi S, Civitarese AE, Hulver MW, Thoresen GH, Rustan AC \& Smith SR. (2011). Remodeling lipid metabolism and improving insulin responsiveness in human primary myotubes. PLoS One 6, e21068.

Sun C, Zhang F, Ge X, Yan T, Chen X, Shi X \& Zhai Q. (2007). SIRT1 improves insulin sensitivity under insulin-resistant conditions by repressing PTP1B. Cell Metab 6, 307-319. 
Tarnopolsky MA, Rennie CD, Robertshaw HA, Fedak-Tarnopolsky SN, Devries MC \& Hamadeh MJ. (2007). Influence of endurance exercise training and sex on intramyocellular lipid and mitochondrial ultrastructure, substrate use, and mitochondrial enzyme activity. Am J Physiol-Reg I 292, R1271-R1278.

Timmers S, de Ligt M, Phielix E, van de Weijer T, Hansen J, Moonen-Kornips E, Schaart G, Kunz I, Hesselink MK, Schrauwen-Hinderling VB \& Schrauwen P. (2016). Resveratrol as Add-on Therapy in Subjects With Well-Controlled Type 2 Diabetes: A Randomized Controlled Trial. Diabetes Care 39, 2211-2217.

Timmers S, Konings E, Bilet L, Houtkooper RH, van de Weijer T, Goossens GH, Hoeks J, van der Krieken S, Ryu D, Kersten S, Moonen-Kornips E, Hesselink MKC, Kunz I, Schrauwen-Hinderling VB, Blaak E, Auwerx J \& Schrauwen P. (2011). Calorie restriction-like effects of 30 days of resveratrol supplementation on energy metabolism and metabolic profile in obese humans. Cell Metab 14, 612-622.

van der Made SM, Plat J \& Mensink RP. (2015). Resveratrol does not influence metabolic risk markers related to cardiovascular health in overweight and slightly obese subjects: a randomized, placebo-controlled crossover trial. PLoS One 10, e0118393.

van Loon LJ, Koopman R, Manders R, van der Weegen W, van Kranenburg GP \& Keizer HA. (2004). Intramyocellular lipid content in type 2 diabetes patients compared with overweight sedentary men and highly trained endurance athletes. Am J Physiol Endocrinol Metab 287, E558-565.

van Loon LJ, Koopman R, Stegen JH, Wagenmakers AJ, Keizer HA \& Saris WH. (2003). Intramyocellular lipids form an important substrate source during moderate intensity exercise in endurance-trained males in a fasted state. J Physiol 553, 611-625.

Vosselman MJ, Hoeks J, Brans B, Pallubinsky H, Nascimento EB, van der Lans AA, Broeders EP, Mottaghy FM, Schrauwen P \& van Marken Lichtenbelt WD. (2015). Low brown adipose tissue activity in endurance-trained compared with lean sedentary men. Int J Obes (Lond) 39, 1696-1702.

Wang H, Bell M, Sreenevasan U, Hu H, Liu J, Dalen K, Londos C, Yamaguchi T, Rizzo MA, Coleman R, Gong DW, Brasaemle D \& Sztalryd C. (2011a). Unique Regulation of Adipose Triglyceride Lipase (ATGL) by Perilipin 5, a Lipid Droplet-associated Protein. J Biol Chem 286.

Wang H, Sreenevasan U, Hu H, Saladino A, Polster BM, Lund LM, Gong DW, Stanley WC \& Sztalryd C. (2011b). Perilipin 5, a lipid droplet-associated protein, provides physical and metabolic linkage to mitochondria. J Lipid Res 52, 2159-2168.

Yoshino J, Conte C, Fontana L, Mittendorfer B, Imai S, Schechtman KB, Gu C, Kunz I, Rossi Fanelli F, Patterson BW \& Klein S. (2012). Resveratrol supplementation does not improve metabolic function in nonobese women with normal glucose tolerance. Cell Metab 16, 658-664.

Zhang YJ, Zhao H, Dong L, Zhen YF, Xing HY, Ma HJ \& Song GY. (2019). Resveratrol ameliorates high-fat diet-induced insulin resistance and fatty acid oxidation via ATMAMPK axis in skeletal muscle. Eur Rev Med Pharmacol Sci 23, 9117-9125.

Zhou R, Yi L, Ye XK, Zeng XL, Liu K, Qin Y, Zhang QY \& Mi MT. (2018). Resveratrol Ameliorates Lipid Droplet Accumulation in Liver Through a SIRT1/ATF6-Dependent Mechanism. Cell Physiol Biochem 51, 2397-2420. 


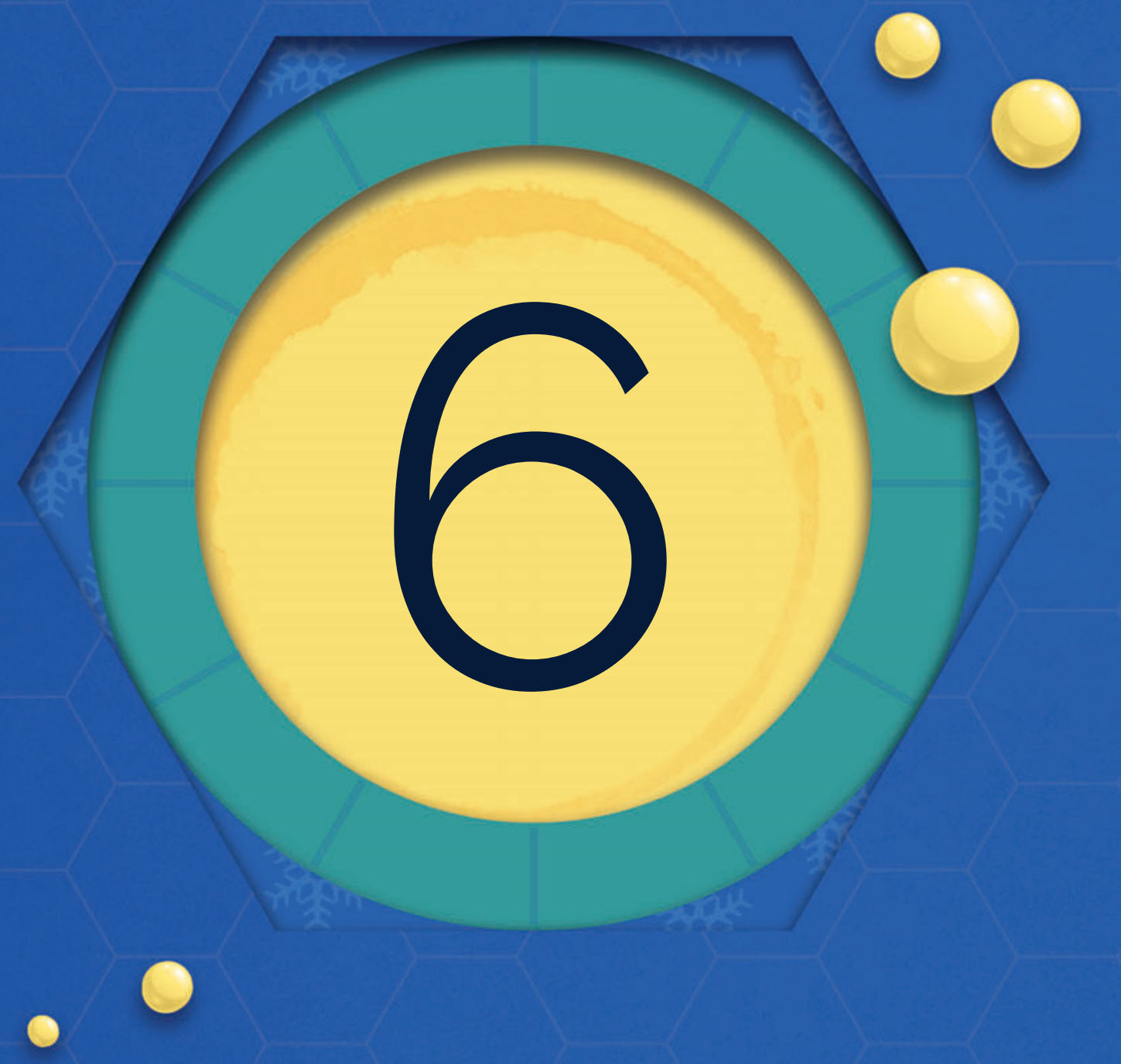




\section{Post-exercise changes in myocellular lipid droplet characteristics of young}

lean individuals are affected by circulatory non-esterified fatty acids

Nynke van Polanen*, Sabine Daemen*, Lena Bilet, Esther Phielix, Esther Moonen-Kornips, Vera B. Schrauwen-Hinderling, Patrick Schrauwen, Matthijs K.C. Hesselink

*These authors contributed equally 


\section{Chapter 6}

\section{Abstract}

Intramyocellular lipid (IMCL) content is an energy source during acute exercise. Nonesterified fatty acid (NEFA) levels can compete with IMCL utilization during exercise. IMCL content is stored as lipid droplets (LDs) that vary in size, number, subcellular distribution and in coating with LD protein PLIN5. Little is known about how these factors are affected during exercise and recovery. Here, we aimed to investigate the effects of acute exercise with and without elevated NEFA levels on intramyocellular LD size and number, intracellular distribution and PLIN5 coating, using highresolution confocal microscopy. In a cross-over study, 9 healthy lean young men performed a 2-hour moderate intensity cycling protocol in the fasted (high NEFA levels) and glucose-fed state (low NEFA levels). IMCL and LD parameters were measured at baseline, directly after exercise and $4 \mathrm{~h}$ post-exercise. We found that total IMCL content was not changed directly after exercise (irrespectively of condition), but IMCL increased $4 \mathrm{~h}$ post-exercise in the fasting condition, which was due to an increased number of LDs rather than changes in size. The effects were predominantly detected in type I muscle fibres and in LDs coated with PLIN5. Interestingly, subsarcolemmal, but not intermyofibrillar IMCL content, was decreased directly after exercise in the fasting condition and was replenished during the $4 \mathrm{~h}$ recovery period. In conclusion, acute exercise affects IMCL storage during exercise and recovery, particularly in type I muscle fibres, in the subsarcolemmal region and in the presence of PLIN5. Moreover, the effects of exercise on IMCL content are affected by plasma NEFA levels. 


\section{Introduction}

One of the earliest hallmarks of type 2 diabetes (T2DM) is resistance of the peripheral tissues to the action of insulin, generally referred to as insulin resistance. Obesity is the major risk factor for insulin resistance and is characterized by excessive accumulation of fat in the body. Limited expandability of adipose tissue results in spill-over of fatty acids (FAs) from the adipose tissue into the circulation with accumulation of lipids in non-adipose tissue like muscle as a consequence (Unger et al., 2010). Intramyocellular lipid (IMCL) content is associated with the development of insulin resistance (Krssak et al., 1999).

While IMCL content can be detrimental for insulin sensitivity, it also serves as an energy source during muscle contraction. Prolonged exercise training results in elevated IMCL content (Schrauwen-Hinderling et al., 2003a). Likewise, endurance trained athletes are characterized by elevated IMCL levels (Goodpaster et al., 2001; Daemen et al., 2018), probably serving as an increased intracellular pool of FAs to fuel oxidation during prolonged exercise. Using palmitate-labelled stable isotope tracers, it has been demonstrated that IMCL is indeed utilized during exercise (Martin et al., 1993; Romijn et al., 1993; Phillips et al., 1996; Coyle et al., 1997; van Loon et al., 2003; Stellingwerff et al., 2007). Some studies have shown that utilization of IMCL during acute exercise results in reduced IMCL content as measured directly after exercise by proton magnetic resonance spectroscopy ( $\left.{ }^{1} \mathrm{H}-\mathrm{MRS}\right)$ or in muscle biopsies (biochemically or histochemically) (Brechtel et al., 2001; Schrauwen-Hinderling et al., 2003b; Stellingwerff et al., 2007; Vermathen et al., 2012; Egger et al., 2013; Shepherd et al., 2013; Bucher et al., 2014). Other acute exercise studies, however, failed to detect a drop in IMCL content (Kiens \& Richter, 1998; Rico-Sanz et al., 1998; Bergman et al., 1999; Roepstorff et al., 2002). These seemingly contrasting results may originate from differences in the methodology used (biochemically, histologically or non-invasively via MRS) as well as from differences in non-esterified fatty acid (NEFA) concentrations in the circulation. Upon exercise, stimulation of adipose tissue lipolysis occurs and plasma NEFA levels increase. Plasma derived NEFAs compete with IMCL-derived FAs for oxidation but may also refuel IMCL stores during exercise and during the 


\section{Chapter 6}

post-exercise period. In inactive muscle, it has indeed been reported that elevated plasma NEFA levels augment IMCL content (Schrauwen-Hinderling et al., 2003b; Hoeks et al., 2010). This indicates that elevated plasma NEFA levels promote incorporation of FAs in IMCL, independent of the need of FAs for oxidation.

Many studies examining the effect of exercise on IMCL content have used ${ }^{1} \mathrm{H}-\mathrm{MRS}$ or biochemical triglyceride extraction to determine IMCL content. However, these methodologies do not take lipid droplet (LD) distribution and morphology into account. IMCL is largely stored in LDs of which the pattern of storage is affected by training status and disease state. Interestingly, we have recently shown that in endurance athletes, IMCL is dispersed in numerous small LDs, specifically in the intermyofibrillar (IMF) region of type I fibres while in T2DM patients most IMCL is stored in large LDs, predominantly in the subsarcolemmal (SS) region of type II muscle fibres (Daemen et al., 2018). This pattern fits with IMCL being an important source of fuel during exercise, as hypothetically, LDs in the IMF region may primarily serve to deliver FAs to mitochondria for oxidation. Hence, it can be hypothesized that IMF LDs would reduce in number and/or size upon exercise. On the other hand, SS LDs are less likely to fuel contraction during exercise but might be prone to grow in size and/or number due to their vicinity to the capillary bed and the resulting exposure to high circulating NEFA levels, such as may be the case in T2DM (which is characterized by high NEFA levels). These putative region and fibre type specific effects may also blunt the overall effect of exercise on total IMCL content. As of now, only one study has examined region specific effects of exercise and recovery on IMCL content and LD size and number (Jevons et al., 2020). This study showed a more profound lowering effect of exercise on IMF LDs than on SS LDs upon 4 hours of exercise at $56 \% \mathrm{VO}_{2} \mathrm{Max}$ in trained athletes (Jevons et al., 2020), however in this study NEFA levels were not reported.

Perilipin 5 (PLIN5) is a LD coat protein involved in LD dynamics by affecting uptake, retention and release of FAs from LDs to mitochondria for oxidation, depending on demand, nutrition or training status (Bosma et al., 2013; Shepherd et al., 2017; Gemmink et al., 2018b). We previously observed that fasting-induced increases in 
plasma NEFA levels resulted in preferential storage of FAs in PLIN5 coated LDs (Gemmink et al., 2016). Others have shown that during moderate intensity exercise, PLIN5 coated LDs are more prone to reduce size compared to LDs devoid of PLIN5 (Shepherd et al., 2013). Together, this suggests a role for PLIN5 post-exercise and during high NEFA levels. Here, we examined the effect of acute exercise (moderate intensity, i.e. $50 \%$ of pre-determined maximal power output) on LD size and number while taking muscle fibre type and subcellular LD distribution into account, and making the distinction between LDs coated with PLIN5 (PLIN5+) and those devoid of PLIN5 (PLIN5-). Furthermore, to investigate the effects of circulatory NEFA levels on exercise mediated oxidation and post-exercise replenishment of myocellular LDs, the study was performed in both the glucose-fed (low NEFA levels) and the fasted state (high NEFA levels), along with measurements of glucose and fat oxidation.

\section{Methods}

\section{Participants}

Nine healthy lean young men participated in this study. Participation in competitive sports ( $>2$ hours per week), an unstable body weight ( $>3 \mathrm{~kg}$ change in preceding six months), medication use or any medical condition requiring treatment were exclusion criteria. The institutional medical ethics committee approved the study in accordance with the Declaration of Helsinki, and all participants gave their informed written consent. The study is registered as NCT01906333 at https://clinicaltrials.gov. Participant characteristics are shown in Table 1. 


\section{Chapter 6}

Table 1. Participant characteristics

\begin{tabular}{lc}
\hline Characteristics & \\
\hline Age (years) & $23.2 \pm 2.2$ \\
Height $(\mathrm{m})$ & $1.85 \pm 0.09$ \\
Weight $(\mathrm{kg})$ & $77.9 \pm 9.5$ \\
$\mathrm{BMI}\left(\mathrm{kg} / \mathrm{m}^{2}\right)$ & $22.7 \pm 1.8$ \\
Fat mass $(\%)$ & $14.4 \pm 5.5$ \\
$\mathrm{VO}_{2 \max }\left(\mathrm{ml} \mathrm{O}_{2} / \mathrm{kg} / \mathrm{min}\right)$ & $43.3 \pm 6.1$ \\
\hline
\end{tabular}

Data are means $\pm S D(n=9) ; B M I, B o d y$ Mass Index; $V_{2}$ max, maximal aerobic capacity.

\section{Study protocol}

At the beginning of the study, body composition (hydrostatic weighing) and maximal aerobic capacity $\left(\mathrm{VO}_{2} \mathrm{max}\right)$ were determined in all participants. The experimental trial comprised two test days separated by at least one week and were performed in random order. Participants refrained from physical activity two days prior to the test days and consumed a standardized meal the evening prior to the test days. On the test days, participants reported to the laboratory after an overnight fast (no caloric intake after 10 PM the preceding day). A muscle biopsy was taken from the $\mathrm{m}$. vastus lateralis under local anaesthesia (2\% lidocaine), according to the Bergstrom technique (Bergstrom et al., 1967). The biopsy was immediately frozen in melting isopentane and stored at $-80^{\circ} \mathrm{C}$ for later immunohistochemical analysis. Thereafter, a Teflon cannula was inserted into an antecubital forearm vein for sampling of blood and participants rested for $30 \mathrm{~min}$. At baseline $(\mathrm{t}=-60)$, the participants ingested either 1.4 $\mathrm{g} / \mathrm{kg}$ body weight of glucose (dissolved in water to a $20 \%$ solution and flavoured with $1 \mathrm{ml}$ lemon juice) or the same amount of plain water. After this, participants started exercising on a stationary bike at $50 \%$ of their pre-determined maximal power output (Wmax) ( $\mathrm{t}=0 \mathrm{~min}$ ) for two hours. During exercise, blood samples were drawn and substrate oxidation was measured by indirect calorimetry (Omnical, Maastricht, the Netherlands) every $30 \mathrm{~min}$ for $15 \mathrm{~min}$ ( $\mathrm{at} \mathrm{t}=30,60,90$ and $120 \mathrm{~min}$ ) with continuous heart rate monitoring. Participants consumed the glucose or water drink every 30 min during exercise. Immediately upon cessation of exercise, a second muscle biopsy was taken. Subsequently, participants bed-rested for $4 \mathrm{~h}$, followed by a third muscle 
biopsy $4 \mathrm{~h}$ post-exercise. During the $4 \mathrm{~h}$ post-exercise period, drinks (glucose or plain water) were consumed hourly and blood samples were drawn (prior to the drinks) and measurements of substrate oxidation were performed by indirect calorimetry for $30 \mathrm{~min}$ (at $\mathrm{t}=180,240,300$ and $360 \mathrm{~min}$ ). Fat oxidation and substrate oxidation was calculated from $\mathrm{VO}_{2}$ and $\mathrm{VCO} 2$ using the formulas of Peronnet (Peronnet \& Massicotte, 1991). The experimental design is depicted in Figure 1.

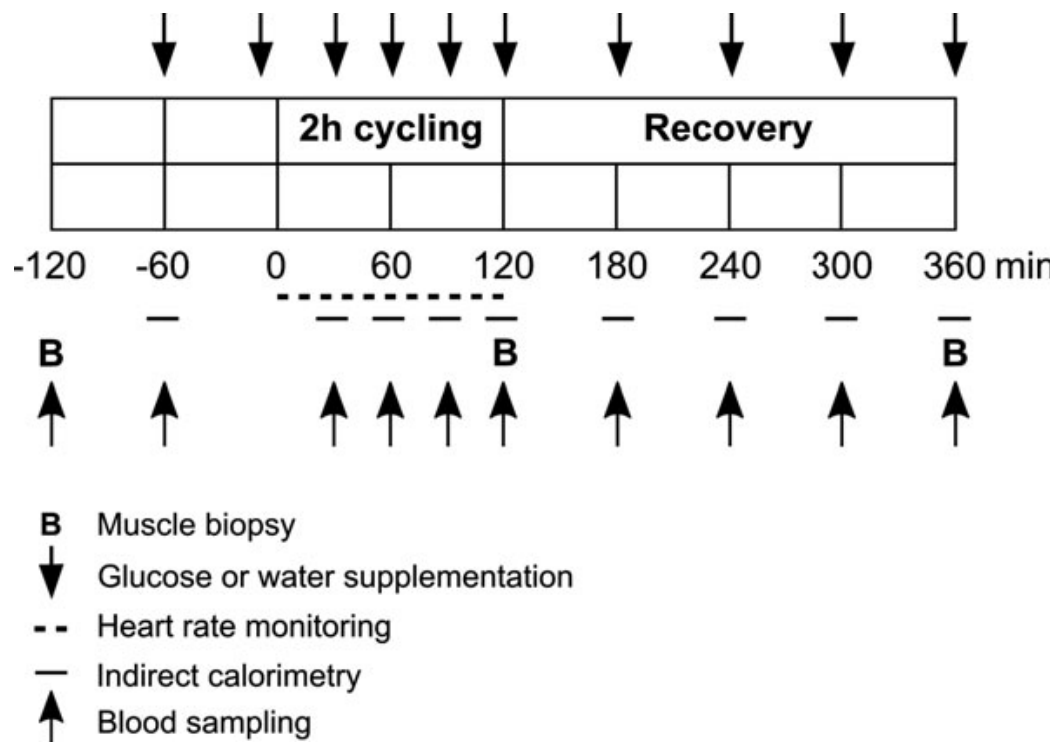

Figure 1. Experimental design. The experimental design of the study. All participants performed the protocol twice, once in the fasted state consuming water and once with glucose supplementation, with at least one week in between.

\section{Measurements prior to test days}

A routine incremental cycling test on a stationary bike until exhaustion was used to determine maximal exercise capacity as described previously (Kuipers et al., 1985) and hydrostatic weighing with simultaneous measurement of lung volume was performed to determine body composition. The equation of Siri (Siri, 1956) was used to calculate fat percentage, fat mass and fat-free mass. 


\section{Chapter 6}

\section{Blood sample analysis}

Blood samples were collected in EDTA-containing tubes and immediately spun at high speed and frozen in liquid nitrogen and subsequently stored at $-80^{\circ} \mathrm{C}$ until assayed. Plasma NEFAs and glucose were measured with enzymatic assays automated on a Cobas Fara/Mira (NEFA: Wako Nefa C test kit; Wako Chemicals, Neuss, Germany) (glucose: hexokinase method; Roche, Basel, Switzerland).

\section{Histochemical analysis}

From the muscle biopsies, $5 \mu \mathrm{m}$ thick sections were cut and mounted on glass slides. To exclude effects of staining variability, all samples from one participant were mounted on the same glass slide. Immunohistochemical stainings were performed as previously described (Daemen et al., 2018). Primary antibodies against laminin (L9393; Sigma-Aldrich, Zwijndrecht, the Netherlands; 1:25), myosin heavy chain type I (A4.840; Developmental Studies Hybridoma Bank, Iowa, USA; 1:25) and PLIN5 (GP31; Progen Biotechnik, Heidelberg, Germany; 1:25) were used. Appropriate secondary antibodies were conjugated with Alexa Fluor 405, Alexa Fluor 555 or Alexa Fluor 647. LDs were stained with Bodipy 493/503 (D3922; Molecular Probes, Thermo Fisher Scientific, Landsmeer, the Netherlands; 1:100).

\section{Image acquisition and analysis}

Images were taken with a Leica TCS SP8 STED microscope (Leica Microsystems, Amsterdam, the Netherlands), in confocal mode with similar settings as described previously (Daemen et al., 2018). For each time point of each participant, images of twenty fibres in cross-sectional view were taken with appropriate type I to type II fibre ratio, determined as previously described (Daemen et al., 2018). Images were deconvolved using Huygens Professional software (Scientific Volume Imaging B.V., Hilversum, the Netherlands). IMCL, LD size, number and distribution were analysed with ImageJ (NIH, Bethesda, Maryland, USA) and MATLAB (The MathWorks Inc., Natick, Massachusetts, USA). IMCL was determined by total lipid area (Bodipy staining)/cell area (\% lipid area fraction), LD number by total LD number/cell area 
$\left(\# / \mu \mathrm{m}^{2}\right)$ and LD size by total lipid area/total LD count $\left(\mu \mathrm{m}^{2}\right)$. This was calculated for all fibres combined (taking into account the pre-determined type I to type II fibre ratio) and in type I and type II fibres separately. As previously explained, the SS region was defined as the outer $8 \%$ of the cell and IMF region as the remaining $92 \%$ using a custom-made MATLAB script (Daemen et al., 2018). Within each region, IMCL, LD number and size was determined for all fibres combined. Finally, LDs were subdivided into LDs coated with PLIN5 (PLIN5+) and LDs devoid of PLIN5 (PLIN5-) using a custom written MATLAB script (Gemmink et al., 2018a). For both PLIN5+ and PLIN5- LDs, number and size was computed for all fibres combined.

\section{Statistics}

Data are reported as mean \pm SEM. Statistical analyses were performed using SPSS version 25 (IBM SPSS Inc., NC, USA). A repeated measures ANOVA was performed with time and treatment (fasting or glucose) as within-subject factors for energy expenditure, substrate oxidation and plasma values of NEFAs and glucose. Thereafter a pairwise comparison was performed with a Bonferroni correction for multiple comparisons. A mixed model was used to compare IMCL content, LD size, number and PLIN5 coating. In case of a time effect, paired t-tests were performed between time points for both the glucose and fasting condition. If there was a significant treatment effect, glucose and fasting were compared at each time point using paired $\mathrm{t}$-tests. Statistical significance was set a priori at $\mathrm{p}<0.05$.

\section{Results}

\section{Plasma non-esterified fatty acids and glucose}

There was a significant time $(\mathrm{p}<0.001)$, treatment $(\mathrm{p}<0.001)$ and time $\mathrm{e}^{*}$ treatment interaction $(p<0.001)$ effect for plasma NEFA concentrations. Plasma NEFA concentrations were substantially higher post-exercise $(p<0.001)$ and 4 h postexercise in the fasted state $(\mathrm{p}=0.001)$ compared with the glucose-fed state (Figure 2A). Moreover, plasma NEFA concentrations were increased at the end of exercise 


\section{Chapter 6}

$(\mathrm{p}<0.001)$ and $4 \mathrm{~h}$ post-exercise $(\mathrm{p}=0.025)$ in the fasted condition compared with baseline, whereas plasma NEFA concentrations were decreased $4 \mathrm{~h}$ post-exercise $(t=360)$ in the glucose-fed state compared with baseline $(p=0.047$, Figure $2 A)$.

During the entire experimental testing, plasma glucose concentrations remained within the normoglycemic range. Plasma glucose concentrations showed a significant treatment $(\mathrm{p}=0.002)$ and time*treatment interaction $(\mathrm{p}=0.008)$ effect. Plasma glucose concentrations were higher directly post-exercise $(\mathrm{p}=0.002)$ and $4 \mathrm{~h}$ post-exercise $(\mathrm{p}=0.008)$ in the glucose-fed state compared with the fasted state (Figure 2B). Moreover, plasma glucose concentrations were increased at the end of exercise $(t=120)$ compared with baseline $(t=-60)$ in the glucose-fed state $(p=0.029)$, whereas plasma glucose concentrations were decreased $4 \mathrm{~h}$ post-exercise in the fasted state compared with baseline ( $\mathrm{p}=0.003$, Figure $2 \mathbf{B})$.
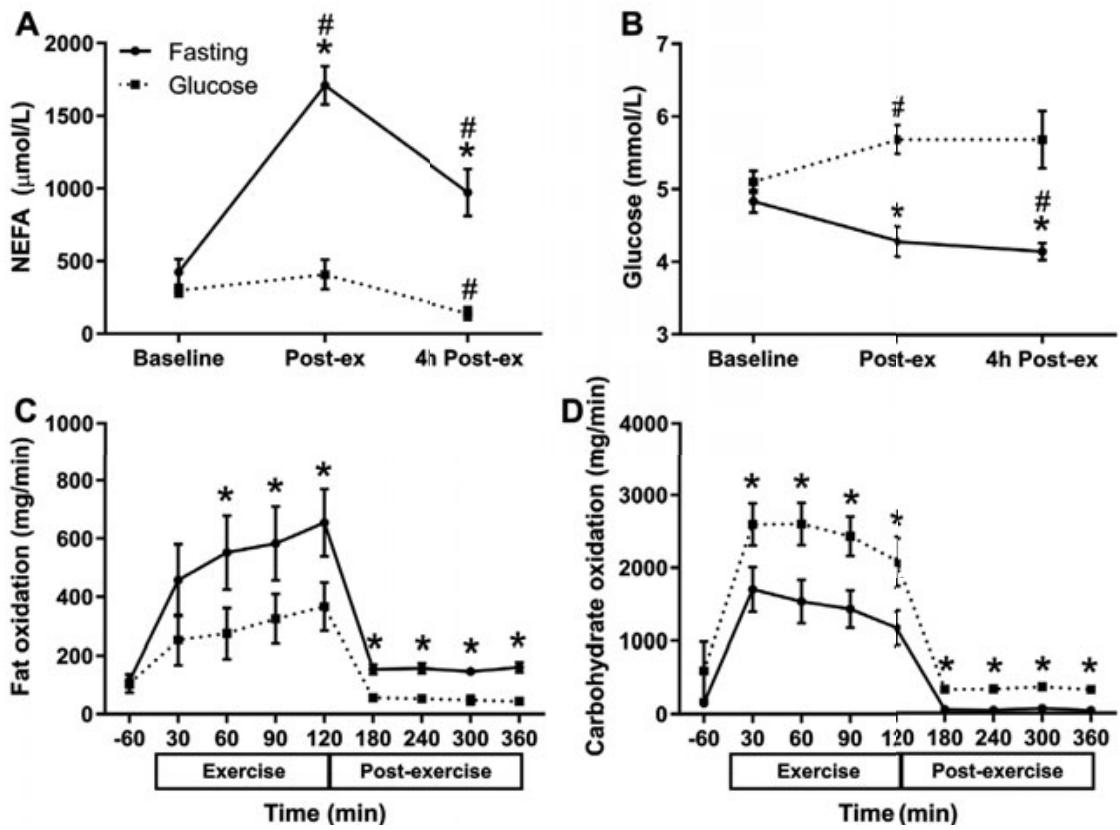

Figure 2. Differential substrate levels and usage with fasting versus glucose supplementation. Plasma non-esterified fatty acids (NEFA) levels (A), plasma glucose levels (B), fat oxidation (C) and carbohydrate oxidation (D) before, during and post-exercise with (dotted line/squares) and without (solid line/circles) glucose supplementation. * $p<0.05$ compared with glucose-fed state, \# $p<0.05$ compared with baseline $(t=-60 \mathrm{~min})$. Data are mean $\pm \operatorname{SEM}(n=9)$. 


\section{Substrate oxidation}

During exercise, there was a significant time $(\mathrm{p}<0.001)$ and treatment $(\mathrm{p}=0.042)$ effect for fat oxidation and a significant treatment $(\mathrm{p}=0.018)$ effect for carbohydrate oxidation (and a tendency for a time effect $(\mathrm{p}=0.082)$ ). This was reflected in a higher carbohydrate oxidation and lower fat oxidation in the glucose-fed state compared to the fasted state $(\mathrm{p}<0.05$, Figure $2 \mathrm{C}+\mathrm{D})$. In the post-exercise period, a significant treatment effect was observed for both fat $(\mathrm{p}=0.004)$ and carbohydrate $(\mathrm{p}=0.011)$ oxidation. Carbohydrate oxidation was higher and fat oxidation was lower in the glucose-fed state versus the fasting condition during all time points of the recovery period ( $<<0.05$, Figure $2 C+D$ ). Surprisingly, we observed that energy expenditure during exercise in the fasted state was modestly, but significantly, lower than in the glucose-fed state ( $p<0.05$, Table 2$)$. Two individuals were unable to maintain the workload demanded throughout the full two hours, if fasted. Thus, workload was lowered to facilitate completion of the trial, which might explain the differences in EE during exercise. Post-exercise, all subjects rested and energy expenditure was similar between the two conditions ( $p>0.05$, Table 2$)$.

Table 2. Energy expenditure during and after exercise in glucose-fed and fasted state.

\begin{tabular}{lccc}
\hline Treatment & & Glucose & Fasted \\
\hline \multirow{2}{*}{ Exercise } & Time (min) & EE (kJ/min) & EE (kJ/min) \\
& 30 & $51.0 \pm 3.0$ & $45.0 \pm 2.6^{*}$ \\
Post-exercise & 50 & $51.8 \pm 3.2$ & $46.0 \pm 2.9^{*}$ \\
& 120 & $52.3 \pm 3.2$ & $45.6 \pm 2.9^{*}$ \\
& 180 & $51.4 \pm 3.5$ & $44.2 \pm 3.0^{*}$ \\
& 240 & $7.5 \pm 0.5$ & $6.9 \pm 0.4$ \\
& 300 & $7.1 \pm 0.4$ & $6.9 \pm 0.4$ \\
& 360 & $7.3 \pm 0.4$ & $6.8 \pm 0.5$ \\
\end{tabular}

Data are means $\pm S E M, n=9$ during exercise, $n=7$ during post-exercise. EE, energy expenditure; ${ }^{*} p<0.05$ compared with glucose-fed state. 


\section{Intramyocellular lipid content and lipid droplet morphology}

Total IMCL content (based on type I and II fibres together) displayed a significant time $(p=0.013)$ and treatment effect $(p=0.007)$. Irrespective of the fed or fasted condition, IMCL content was not significantly changed from baseline, directly post-exercise (Figure 3A). At the same time point, however, LD size appeared modestly reduced directly post-exercise in the fasted state $(p=0.004$, Figure $3 B$ ) but not in the glucosefed state. Four hours post-exercise, however, IMCL content significantly $(p=0.036)$ increased in the fasting condition compared to directly post exercise, which resulted in a significantly higher IMCL content in the fasted state than in glucose-fed state $4 \mathrm{~h}$ post-exercise $(p=0.016$, Figure 3A). LD number displayed a tendency $(p=0.084)$ for a time effect. The increase in IMCL content in the fasted state during the recovery period appeared to result from an increased number of LDs (Figure 3C) rather than an increase in LD size ( $p=0.754$, Figure 3B). These observations are also visualized in Figure 3J which displays type I fibres of the same participant $4 \mathrm{~h}$ post-exercise in both fasting and glucose-fed state.

When distinguishing between fibre types, the elevated IMCL content $4 \mathrm{~h}$ post-exercise in the fasted condition compared to the glucose-fed state was only observed in the type I fibres ( $p=0.012$, Figure 3D). Higher total IMCL content 4 h post-exercise is more likely to originate from the near significant increase $(p=0.058)$ of IMCL directly postexercise than from the modest non-significant $(\mathrm{p}=0.841)$ drop in the glucose-fed state. Higher IMCL content in type I fibres $4 \mathrm{~h}$ post-exercise was paralleled by an increase in the number of LDs ( $p=0.010$, Figure 3F) while LD size did not change $(p=0.602$, Figure 3E). Similar to the overall picture, exercise in the fasted condition reduced LD size in type I fibres ( $p=0.002$, Figure 3E). No significant effects of exercise on IMCL content, LD number nor size were observed in type II fibres (Figure 3G-I). Thus, the effects of exercise on IMCL seems to be mainly due to effects in type I fibres. Of note, in these untrained participants, type I and type II fibres represented $42 \pm 3.1 \%$ and $58 \pm 3.1 \%$ of total fibres, respectively. 

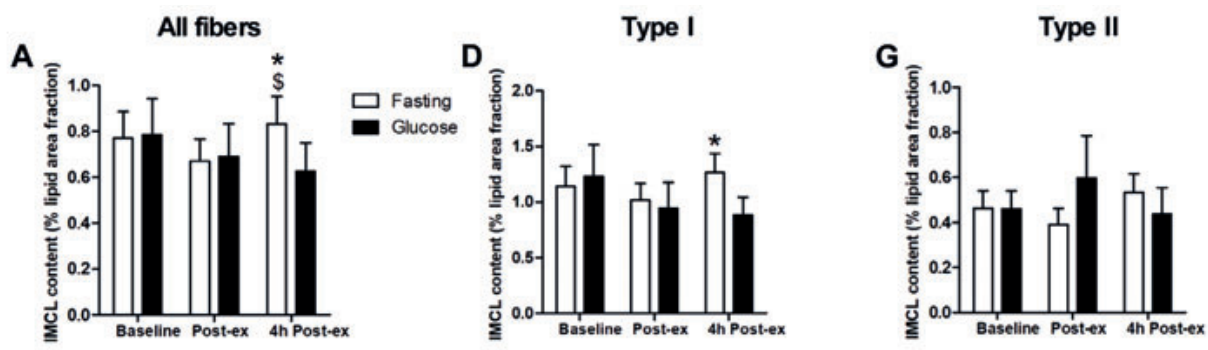

B

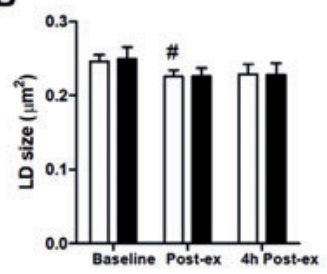

C

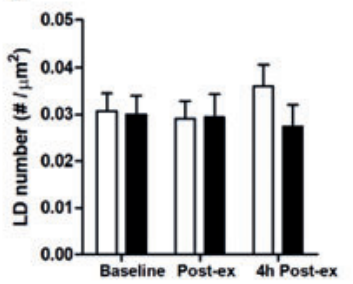

J

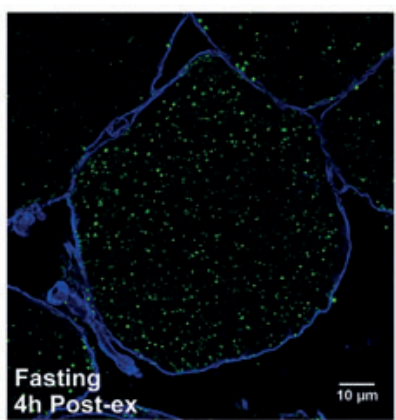

E

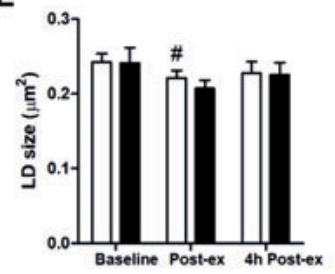

F
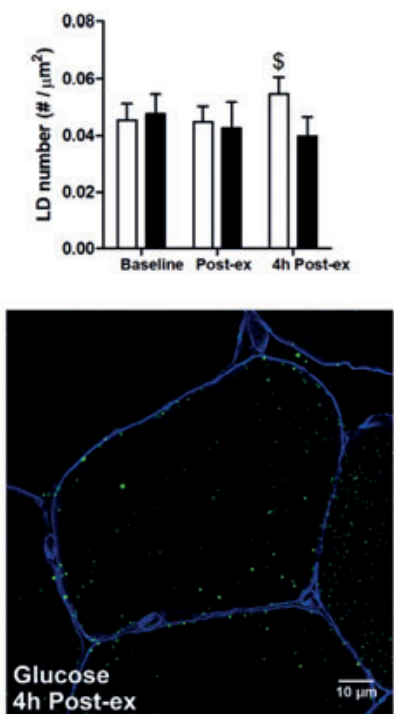

H

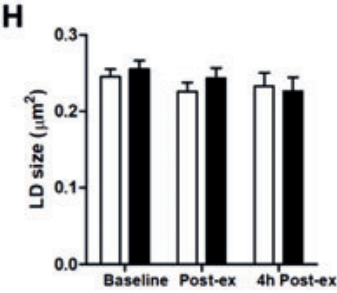

I

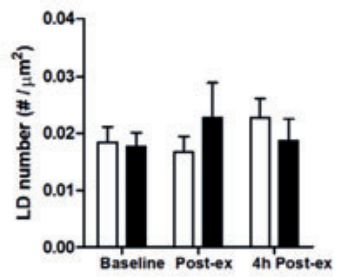

Figure 3. Increased IMCL content 4 h post-exercise with fasting. Intramyocellular lipid (IMCL) content $(\boldsymbol{A}, \boldsymbol{D}, \boldsymbol{G})$, lipid droplet $(L D)$ size $(\boldsymbol{B}, \boldsymbol{E}, \boldsymbol{H})$ and $L D$ number $(\boldsymbol{C}, \boldsymbol{F}, \boldsymbol{I})$ in all fibres, type I and type II fibres. Representative images of type I fibres of the same participant $4 \mathrm{~h}$ post-exercise in fasting condition or with glucose-supplementation $(\boldsymbol{J})$. * $p<0.05$ compared with glucose-fed state; \# $p<0.05$ compared with baseline; $\$$ compared with post-exercise. Data are mean \pm SEM $(n=9)$. 


\section{Chapter 6}

\section{Cellular distribution of lipid droplets}

We next investigated if exercise differentially affected IMCL content, LD size and LD number in the SS vs the IMF region. Contrary to our expectations, and in the absence of significant changes in total IMCL content, we observed a significant decrease in IMCL content in the SS region directly after exercise in the fasting condition $(p=0.012$, Figure 4A). The decrease in SS IMCL content originated from a decrease in LD size ( $p=0.005$, Figure 4B) as well as in LD number $(p=0.040$, Figure $4 C)$. Four hours postexercise, SS IMCL content was replenished in the high NEFA (fasted) condition $(\mathrm{p}=0.028$, Figure 4A), mainly due to an increase in LD number (Figure 4C, $\mathrm{p}=0.040$ ). As a result, IMCL content in the SS region was significantly higher in the fasted vs the glucose-fed state $4 \mathrm{~h}$ post-exercise ( $\mathrm{p}=0.005$, Figure $4 \mathrm{~A}$ ). In the IMF region, no significant differences in IMCL content or LD number and size in the IMF region were detected (Figure 4D-F).

\section{PLIN5 coating of lipid droplets}

LDs can be separated into pools of LDs decorated with PLIN5 (PLIN5+ LDs) and those devoid of PLIN5 (PLIN5- LDs) (Figure 5H). Interestingly, we observed a time $(p=0.007)$ and treatment effect $(p=0.004)$ for IMCL content in PLIN5+ LDs (Figure 5A). Four hours post-exercise, IMCL content in PLIN5+ LDs was significantly higher in the fasting state compared to the glucose-fed state $(\mathrm{p}=0.013)$ (Figure $\mathbf{5 A + G})$. This was predominately due to a tendency $(p=0.062)$ for an increase in lipid content in the fasting condition during the recovery period, rather than due to a drop in IMCL content in the glucose fed state (Figure 5A, p=0.534). The higher IMCL content in the fasted state, 4 h post-exercise, seems to originate from a higher LD number (Figure 5C), rather than from differences in LD size (Figure 5B). For PLIN5- LDs we could not detect any significant changes in IMCL content, LD size or number with exercise or during the recovery period (Figure 5D-F). 

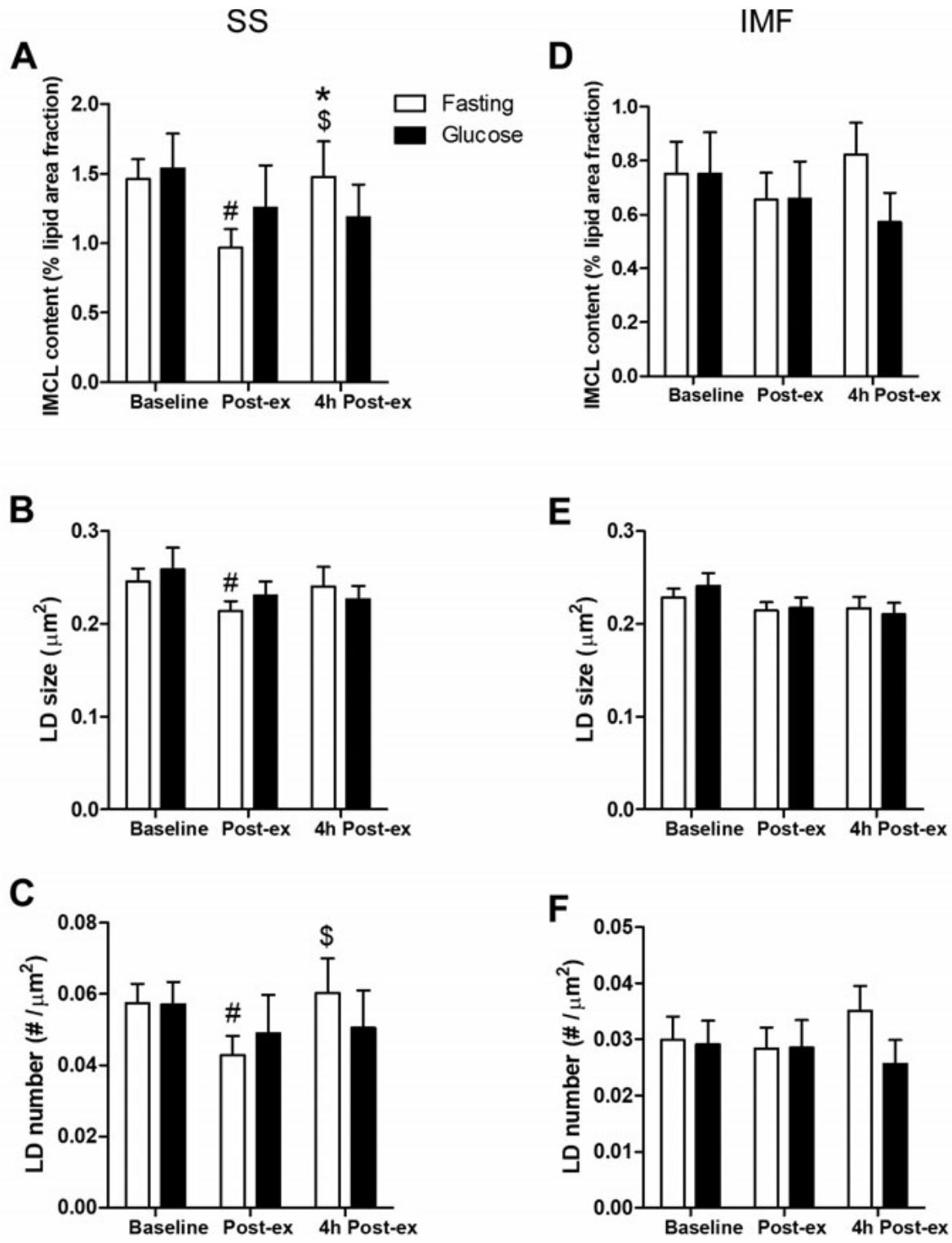

Figure 4. Subsarcolemmal IMCL content transiently decreases directly post-exercise and is replenished during recovery in the fasted state. IMCL content $(A, D), L D$ size $(B, E)$ and $L D$ number $(\boldsymbol{C}, \boldsymbol{F})$ in all fibres in the subsarcolemmal (SS) or intermyofibrillar (IMF) region of the muscle fibre. ${ }^{*} p<0.05$ compared with glucose-fed state; \# $p<0.05$ compared with baseline; \$ compared with post-exercise. Data are mean \pm SEM $(n=9)$. 

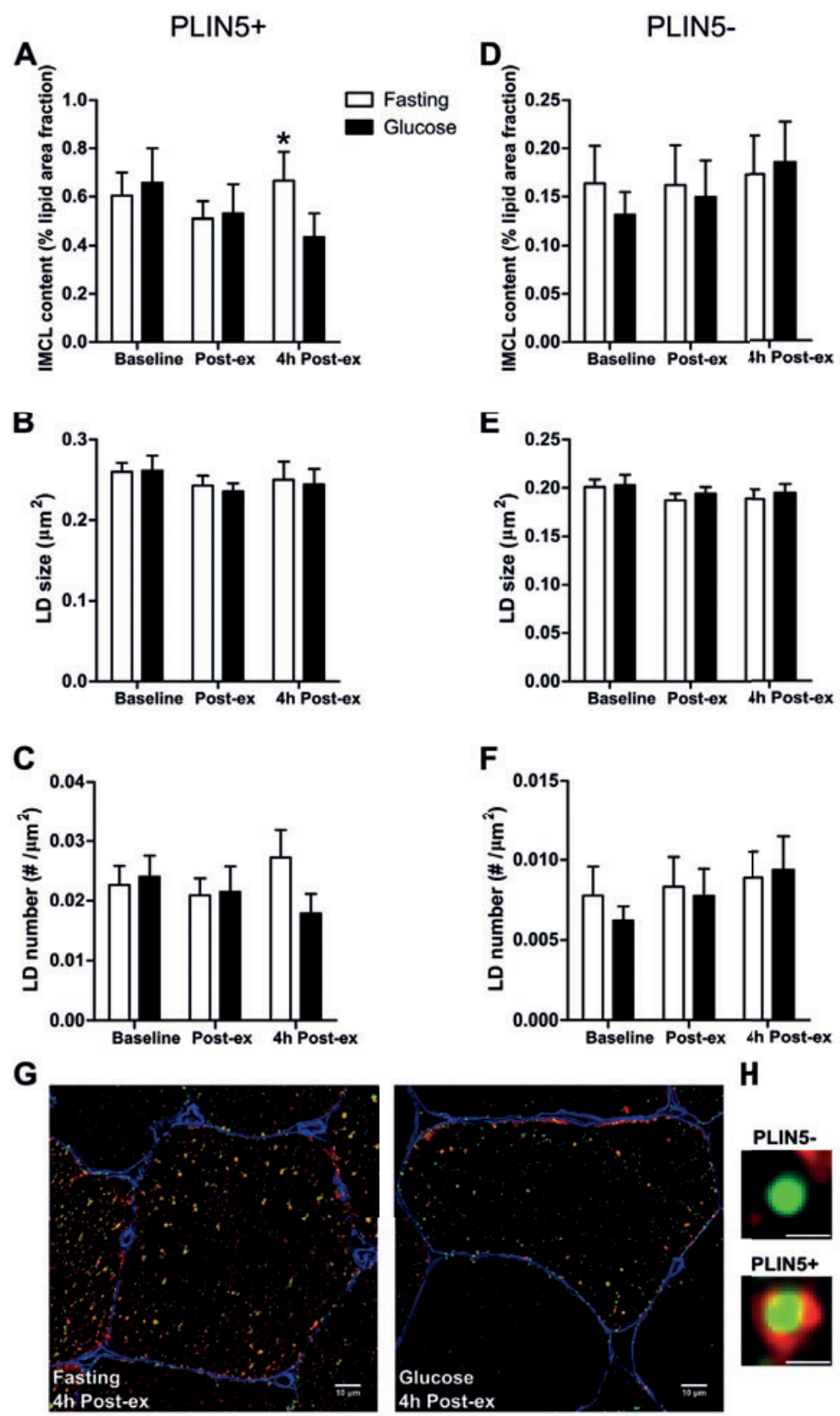

Figure 5. Increased IMCL content 4 h post-exercise in the fasting state was specifically in lipid droplets coated with PLIN5. IMCL content $(\boldsymbol{A}, \boldsymbol{D}), L D$ size $(\boldsymbol{B}, \boldsymbol{E})$ and $L D$ number $(\boldsymbol{C}, \boldsymbol{F})$ in all fibres in LDs coated with PLIN5 (PLIN5+) or devoid of PLIN5 (PLIN5-). Representative images of type I fibres of the same participant $4 \mathrm{~h}$ post-exercise in fasting condition or with glucose-supplementation in PLIN5+ LDs (G). Example of a PLIN5- and a PLIN5+ LD. Scale bar $=1 \mu \mathrm{m}(\boldsymbol{H}) .{ }^{*} p<0.05$ compared with glucose-fed state. Data are mean \pm SEM $(n=9)$. 


\section{Discussion}

Data on the effect of acute exercise on IMCL content are ambiguous. The different and sometimes contradictory data on post-exercise IMCL content may originate from a variety of factors, including muscle fibre typology, subcellular location of the stored fat and nutritional status, or more specifically, circulatory NEFA levels. Here, we examined the effect of an acute bout of exercise on IMCL content and distribution in healthy lean young men under a fasted and glucose-fed condition. It was observed that the effect of an acute bout of exercise on IMCL is affected by nutritional status during and after exercise, probably related to plasma NEFA levels. Changes in IMCL content upon exercise were muscle fibre type and region specific and dependent on the presence of PLIN5 on the LD. We observed that IMCL content decreased in the SSbut not in the IMF- region upon acute exercise and that $4 \mathrm{~h}$ following exercise, IMCL content was elevated in the fasted but not in the glucose-fed state. These significant changes were restricted to type I fibres, LDs decorated with PLIN5 and under fasted conditions, when plasma NEFA levels were highest.

In contrast to the glucose-fed state, moderate intensity exercise in the fasted state profoundly elevated plasma NEFA levels and increased fat oxidation, similar to what we reported previously using the same study design (Bilet et al., 2011; Bilet et al., 2015). We previously showed that the increase in plasma NEFA content and fat oxidation in the fasted state upon exercise was paralleled by increased cardiac (Bilet et al., 2011) and hepatic (Bilet et al., 2015) fat content $4 \mathrm{~h}$ post-exercise. This increase in ectopic fat accumulation was completely blunted upon consumption of a glucose drink during and after exercise, which resulted in blunted NEFA levels and fat oxidation rates. These observations in heart and liver are in line with the results of the current study, revealing restoration of IMCL $4 \mathrm{~h}$ post-exercise in the fasted, but not the glucose-fed state. This was observed as well in trained athletes who were fasted during and after exercise (Jevons et al., 2020). We suggest that these findings may be related to higher plasma NEFA levels (and lower fat oxidation), which we report in parallel with the changes in IMCL. However, we cannot exclude that other factors, such as higher glucose flux or higher insulin levels in the glucose-fed condition, may be involved in 


\section{Chapter 6}

the differential effects of exercise in the fed or fasted state on IMCL content $4 \mathrm{~h}$ post exercise. In addition, timing of the measurements could be a factor in the results we obtained, as we have recently shown that IMCL displays $24 \mathrm{~h}$ rhythmicity under resting conditions in healthy volunteers (Held et al., 2020). It should be noted that in the current study, the timespan of the entire protocol, i.e. exercise and recovery, was approximately 8 hours and that we used a cross-over design where volunteers were their own controls and both arms were carried out at the same time points. Therefore, although we cannot exclude a small effect of the diurnal rhythm of IMCL content on our findings, such an effect likely is insufficient to explain the difference we observe between the glucose-fed and fasted state.

In the fasted state, we observed a transient non-significant drop in IMCL content during exercise with an increase in IMCL in the recovery phase, resulting in higher IMCL levels in the fasted than in the glucose fed state. The higher IMCL levels originated from an increase in number (rather than size) of LDs, similar to was has been reported by others (Jevons et al., 2020). In line with previous studies (Shepherd et al., 2013; Jevons et al., 2020), the overall changes in IMCL content were detected in type I muscle fibres (that store most IMCL and are predominantly recruited at exercise with this low intensity) but not in type II fibres. These findings are in line with previous observations that trained individuals store a higher number of LDs in type I muscle fibres compared to sedentary individuals (Daemen et al., 2018). Tracer studies revealed that FAs derived from IMCL contribute to total fat oxidation during exercise (Martin et al., 1993; Romijn et al., 1993; Phillips et al., 1996; Coyle et al., 1997; van Loon et al., 2003). The increased IMCL-derived fat oxidation can result in a drop in IMCL content (Chee et al., 2016) and consequently changes in LD size and number (Jevons et al., 2020). Directly post-exercise, we did not observe a significant drop in total IMCL content in the present study. In the fasted state, fat oxidation rates and plasma NEFA levels were higher throughout exercise and recovery compared to the glucose-fed state. While the methodology used in the present study does not permit statements on FA flux through the LD, it is conceivable that profound elevation of plasma NEFAs in the fasted condition (and the concomitant increased NEFA delivery 
to the muscle) may refuel the LDs during exercise, hence preventing a significant drop in IMCL. Indeed, re-esterification of fat (Sacchetti et al., 2002) and increased IMCL synthesis (Bergman et al., 1999) in exercising muscle have been shown. As expected, glucose levels and carbohydrate oxidation levels were much higher in the glucose-fed state, indicating that carbohydrates were the main energy source in the glucose-fed condition.

In that respect, it is interesting to note that exercise in the fasted state resulted in a transient drop in IMCL content in the SS region, followed by restoration 4 hours post-exercise, whereas no significant effects were observed in the IMF region. LDs in the IMF region and in vicinity of mitochondria supposedly fuel muscle contraction (Hood, 2001). Thus, our observation in lean untrained individuals does not support our hypothesis that IMF LDs would contribute more to lipid oxidation during exercise than SS LDs, and therefore would reduce in size and/or number more profoundly. Our findings also do not match with recent observations in trained athletes (Jevons et al., 2020) and young active individuals (Chee et al., 2016), where it was found that exercise resulted in a drop in IMCL in the IMF region, which was restored $4 \mathrm{~h}$ postexercise (Jevons et al., 2020). On the other hand, our results are in line with previous findings where the number of SS LDs was reduced in the leg muscle of elite skiers after 1 hour cross-country skiing (Koh et al., 2017), however they found a decrease in IMF LD number in the arms. It should be noted, that prolonged exercise training programs reduce SS lipid content (Nielsen et al., 2010; Devries et al., 2013; Li et al., 2014) and trained individuals display a lower SS lipid compared to sedentary individuals (Daemen et al., 2018). The untrained participants in the current study may have higher SS lipid storage compared to athletes, possibly explaining this discrepancy. Another potential explanation for the observation that exercise in the fasted state seems to specifically affect SS LDs may include FA trafficking from SS to IMF LDs; a high IMF LD-derived fat oxidation (with IMF lipid content quantitatively being the larger pool) may trigger FA flux from SS LDs towards IMF LDs. FA transport from the SS to the IMF region is more likely than LD motility in the skeletal muscle due to its organized structure (Bosma, 2016). As both LD number and size decreased in 


\section{Chapter 6}

the present study, a transfer of FAs is expected rather than a transfer of LDs, as the latter would result in a decrease of primarily LD number. However, the importance of lipid oxidation in the SS mitochondria themselves during exercise should also be considered, as exercise training more profoundly affects mitochondrial density and oxidative capacity of SS mitochondria compared to IMF mitochondria (Bizeau et al., 1998; Koves et al., 2005). Regardless the exact mechanism, our data suggests a yet unidentified role for SS LDs in acute exercise and recovery of exercise.

The higher and elevated IMCL levels in fasted state after 4 hours of recovery were detected when plasma NEFA levels were still profoundly elevated, while rates of fat oxidation had dropped substantially. Jointly, these data suggest that IMCL is restored and LDs serve as a sink to store excess FAs, an observation that we showed previously when comparing active and non-active muscle during exercise (SchrauwenHinderling et al., 2003b) or upon prolonged fasting (Gemmink et al., 2016). The storage of excess fat upon prolonged fasting was accounted for specifically by LDs decorated with PLIN5 (PLIN5+ LDs) (Gemmink et al., 2016). PLIN5 is a LD coat protein involved in lipid storage and release of fat for oxidation (for review see (Gemmink et al., 2020)). Others have shown that PLIN5 coated LDs are preferentially used for lipolysis during exercise (Shepherd et al., 2013). The increase in IMCL content after 4 h of recovery in the fasting state, was completely accounted for by PLIN5+ LDs in the present study, suggesting preferential storage of FAs into PLIN5+ LDs post-exercise, which has previously been observed by others as well (Jevons et al., 2020). PLIN5 interacts with enzymes such as ATGL (Granneman et al., 2011; MacPherson et al., 2013; Keenan et al., 2021) and these enzymes may be involved in this regulation of LD turnover as well. In that context, it is interesting to note that we have previously shown that acute exercise leads to a rapid upregulation of PGC1 and PPAR gene expression (Russell et al., 2005), which are transcription factors regulating lipid metabolism gene expression. Interestingly, we previously showed that glucose ingestion during exercise lead to blunted expression of lipid metabolism genes (Civitarese et al., 2005), suggesting that transcriptional regulation may be involved in the regulation of LD turnover. Moreover, it has been observed that HSL preferentially recruits to PLIN5+ LDs after 
acute exercise (Whytock et al., 2018). Jointly, these data indicate involvement of LD coating proteins such as PLIN5, ATGL, HSL, DGAT and other regulation proteins in LD turnover during fasted conditions, and show that PLIN5+ LDs can serve as a sink for excess FAs post-exercise, but also serve to fuel oxidation during exercise (Shepherd et al., 2013; Jevons et al., 2020). These observations match the dual role that has been postulated for PLIN5 in skeletal muscle; regulating FA flux from LD to mitochondria upon metabolic demand (Gemmink et al., 2018b) as well as protecting against lipotoxicity under basal conditions by stimulating lipid storage (Wang et al., 2011; Gemmink et al., 2016).

Our study comes with some limitations especially when it comes to generalizability. Thus, volunteers in our study were lean, young men and it is possible that other results will be found when studying women. Furthermore, our volunteers were relatively untrained and, as also discussed above, other findings can be expected when studying endurance trained athletes. Furthermore, different types of exercise, for example higher intensity exercise, may result in different conclusions regarding the role of SS and IMF lipids in exercise.

In summary, we have shown here that acute exercise affects IMCL storage and LD morphology during exercise and during recovery from exercise, and that the effects of exercise on IMCL content are affected by nutritional status, reflected in higher plasma NEFA levels, and fat oxidation rates. Remarkably, we observed a decrease in lipids stored in the SS region of the muscle fibre acutely after exercise in the fasting condition. During recovery from exercise in the fasting state, IMCL levels increased specifically in type I fibres and in PLIN5+ LDs, predominantly via an increased number of LDs in the SS region. Future studies should aim at unravelling intracellular FAs fluxes during exercise to establish a greater understanding of IMCL metabolism. 


\section{References}

Bergman BC, Butterfield GE, Wolfel EE, Casazza GA, Lopaschuk GD \& Brooks GA. (1999). Evaluation of exercise and training on muscle lipid metabolism. Am J Physiol 276, E106-117.

Bergstrom J, Hermansen L, Hultman E \& Saltin B. (1967). Diet, muscle glycogen and physical performance. Acta physiologica Scandinavica 71, 140-150.

Bilet L, Brouwers B, van Ewijk PA, Hesselink MK, Kooi ME, Schrauwen P \& SchrauwenHinderling VB. (2015). Acute exercise does not decrease liver fat in men with overweight or NAFLD. Sci Rep 5, 9709.

Bilet L, van de Weijer T, Hesselink MK, Glatz JF, Lamb HJ, Wildberger J, Kooi ME, Schrauwen P \& Schrauwen-Hinderling VB. (2011). Exercise-induced modulation of cardiac lipid content in healthy lean young men. Basic research in cardiology 106, 307-315.

Bizeau ME, Willis WT \& Hazel JR. (1998). Differential responses to endurance training in subsarcolemmal and intermyofibrillar mitochondria. Journal of applied physiology $85,1279-1284$.

Bosma M. (2016). Lipid droplet dynamics in skeletal muscle. Experimental Cell Research 340, 180-186.

Bosma M, Sparks LM, Hooiveld GJ, Jorgensen JA, Houten SM, Schrauwen P, Kersten S \& Hesselink MK. (2013). Overexpression of PLIN5 in skeletal muscle promotes oxidative gene expression and intramyocellular lipid content without compromising insulin sensitivity. Biochim Biophys Acta 1831, 844-852.

Brechtel K, Niess AM, Machann J, Rett K, Schick F, Claussen CD, Dickhuth HH, Haering HU \& Jacob S. (2001). Utilisation of intramyocellular lipids (IMCLs) during exercise as assessed by proton magnetic resonance spectroscopy ( $\left.{ }^{1} \mathrm{H}-\mathrm{MRS}\right)$. Hormone and metabolic research $=$ Hormon- und Stoffwechselforschung $=$ Hormones et metabolisme 33, 63-66.

Bucher J, Krusi M, Zueger T, Ith M, Stettler C, Diem P, Boesch C, Kreis R \& Christ E. (2014). The effect of a single $2 \mathrm{~h}$ bout of aerobic exercise on ectopic lipids in skeletal muscle, liver and the myocardium. Diabetologia 57, 1001-1005.

Chee C, Shannon CE, Burns A, Selby AL, Wilkinson D, Smith K, Greenhaff PL \& Stephens FB. (2016). Relative Contribution of Intramyocellular Lipid to Whole-Body Fat Oxidation Is Reduced With Age but Subsarcolemmal Lipid Accumulation and Insulin Resistance Are Only Associated With Overweight Individuals. Diabetes 65, 840-850.

Civitarese AE, Hesselink MK, Russell AP, Ravussin E \& Schrauwen P. (2005). Glucose ingestion during exercise blunts exercise-induced gene expression of skeletal muscle fat oxidative genes. Am J Physiol Endocrinol Metab 289, E1023-1029.

Coyle EF, Jeukendrup AE, Wagenmakers AJ \& Saris WH. (1997). Fatty acid oxidation is directly regulated by carbohydrate metabolism during exercise. Am J Physiol 273, E268-275.

Daemen S, Gemmink A, Brouwers B, Meex RCR, Huntjens PR, Schaart G, Moonen-Kornips E, Jorgensen J, Hoeks J, Schrauwen P \& Hesselink MKC. (2018). Distinct lipid droplet characteristics and distribution unmask the apparent contradiction of the athlete's paradox. Mol Metab 17, 71-81.

Devries MC, Samjoo IA, Hamadeh MJ, McCready C, Raha S, Watt MJ, Steinberg GR \& Tarnopolsky MA. (2013). Endurance training modulates intramyocellular lipid compartmentalization and morphology in skeletal muscle of lean and obese women. 
The Journal of clinical endocrinology and metabolism 98, 4852-4862.

Egger A, Kreis R, Allemann S, Stettler C, Diem P, Buehler T, Boesch C \& Christ ER. (2013). The effect of aerobic exercise on intrahepatocellular and intramyocellular lipids in healthy subjects. PloS one 8, e70865.

Gemmink A, Bosma M, Kuijpers HJ, Hoeks J, Schaart G, van Zandvoort MA, Schrauwen P \& Hesselink MK. (2016). Decoration of intramyocellular lipid droplets with PLIN5 modulates fasting-induced insulin resistance and lipotoxicity in humans. Diabetologia 59, 1040-1048.

Gemmink A, Daemen S, Brouwers B, Huntjens PR, Schaart G, Moonen-Kornips E, Jorgensen J, Hoeks J, Schrauwen P \& Hesselink MKC. (2018a). Dissociation of intramyocellular lipid storage and insulin resistance in trained athletes and type 2 diabetes patients; involvement of perilipin 5? The Journal of physiology 596, 857-868.

Gemmink A, Daemen S, Kuijpers HJH, Schaart G, Duimel H, Lopez-Iglesias C, van Zandvoort M, Knoops K \& Hesselink MKC. (2018b). Super-resolution microscopy localizes perilipin 5 at lipid droplet-mitochondria interaction sites and at lipid droplets juxtaposing to perilipin 2. Biochim Biophys Acta Mol Cell Biol Lipids 1863, 1423-1432.

Gemmink A, Schrauwen P \& Hesselink MKC. (2020). Exercising your fat (metabolism) into shape: a muscle-centred view. Diabetologia 63, 1453-1463.

Goodpaster BH, He J, Watkins S \& Kelley DE. (2001). Skeletal muscle lipid content and insulin resistance: evidence for a paradox in endurance-trained athletes. The Journal of clinical endocrinology and metabolism 86, 5755-5761.

Granneman JG, Moore HP, Mottillo EP, Zhu Z \& Zhou L. (2011). Interactions of perilipin-5 (Plin5) with adipose triglyceride lipase. J Biol Chem 286, 5126-5135.

Held NM, Wefers J, van Weeghel M, Daemen S, Hansen J, Vaz FM, van Moorsel D, Hesselink MKC, Houtkooper RH \& Schrauwen P. (2020). Skeletal muscle in healthy humans exhibits a day-night rhythm in lipid metabolism. Mol Metab 37, 100989.

Hoeks J, van Herpen NA, Mensink M, Moonen-Kornips E, van Beurden D, Hesselink MK \& Schrauwen P. (2010). Prolonged fasting identifies skeletal muscle mitochondrial dysfunction as consequence rather than cause of human insulin resistance. Diabetes 59, 2117-2125.

Hood DA. (2001). Invited Review: contractile activity-induced mitochondrial biogenesis in skeletal muscle. Journal of applied physiology 90, 1137-1157.

Jevons EFP, Gejl KD, Strauss JA, Ortenblad N \& Shepherd SO. (2020). Skeletal muscle lipid droplets are resynthesized before being coated with perilipin proteins following prolonged exercise in elite male triathletes. Am J Physiol Endocrinol Metab 318, E357-E370.

Keenan SN, De Nardo W, Lou J, Schittenhelm RB, Montgomery MK, Granneman JG, Hinde E \& Watt MJ. (2021). Perilipin 5 S155 phosphorylation by PKA is required for the control of hepatic lipid metabolism and glycemic control. J Lipid Res 62, 100016.

Kiens B \& Richter EA. (1998). Utilization of skeletal muscle triacylglycerol during postexercise recovery in humans. Am J Physiol 275, E332-337.

Koh HE, Nielsen J, Saltin B, Holmberg HC \& Ortenblad N. (2017). Pronounced limb and fibre type differences in subcellular lipid droplet content and distribution in elite skiers before and after exhaustive exercise. J Physiol 595, 5781-5795.

Koves TR, Noland RC, Bates AL, Henes ST, Muoio DM \& Cortright RN. (2005). Subsarcolemmal and intermyofibrillar mitochondria play distinct roles in regulating skeletal muscle 
fatty acid metabolism. Am J Physiol Cell Physiol 288, C1074-1082.

Krssak M, Falk Petersen K, Dresner A, DiPietro L, Vogel SM, Rothman DL, Roden M \& Shulman GI. (1999). Intramyocellular lipid concentrations are correlated with insulin sensitivity in humans: a ${ }^{1} \mathrm{H}$ NMR spectroscopy study. Diabetologia 42, 113-116.

Kuipers H, Verstappen FT, Keizer HA, Geurten P \& van Kranenburg G. (1985). Variability of aerobic performance in the laboratory and its physiologic correlates. International journal of sports medicine 6, 197-201.

Li Y, Lee S, Langleite T, Norheim F, Pourteymour S, Jensen J, Stadheim HK, Storas TH, Davanger S, Gulseth HL, Birkeland KI, Drevon CA \& Holen T. (2014). Subsarcolemmal lipid droplet responses to a combined endurance and strength exercise intervention. Physiol Rep 2.

MacPherson RE, Ramos SV, Vandenboom R, Roy BD \& Peters SJ. (2013). Skeletal muscle PLIN proteins, ATGL and CGI-58, interactions at rest and following stimulated contraction. Am J Physiol Regul Integr Comp Physiol 304, R644-650.

Martin WH, 3rd, Dalsky GP, Hurley BF, Matthews DE, Bier DM, Hagberg JM, Rogers MA, King DS \& Holloszy JO. (1993). Effect of endurance training on plasma free fatty acid turnover and oxidation during exercise. Am J Physiol 265, E708-714.

Nielsen J, Mogensen M, Vind BF, Sahlin K, Hojlund K, Schroder HD \& Ortenblad N. (2010). Increased subsarcolemmal lipids in type 2 diabetes: effect of training on localization of lipids, mitochondria, and glycogen in sedentary human skeletal muscle. Am J Physiol Endocrinol Metab 298, E706-713.

Peronnet F \& Massicotte D. (1991). Table of nonprotein respiratory quotient: an update. Can J Sport Sci 16, 23-29.

Phillips SM, Green HJ, Tarnopolsky MA, Heigenhauser GF, Hill RE \& Grant SM. (1996). Effects of training duration on substrate turnover and oxidation during exercise. Journal of applied physiology 81, 2182-2191.

Rico-Sanz J, Hajnal JV, Thomas EL, Mierisova S, Ala-Korpela M \& Bell JD. (1998). Intracellular and extracellular skeletal muscle triglyceride metabolism during alternating intensity exercise in humans. The Journal of physiology 510 ( Pt 2), 615-622.

Roepstorff C, Steffensen CH, Madsen M, Stallknecht B, Kanstrup IL, Richter EA \& Kiens B. (2002). Gender differences in substrate utilization during submaximal exercise in endurance-trained subjects. Am J Physiol Endocrinol Metab 282, E435-447.

Romijn JA, Coyle EF, Sidossis LS, Gastaldelli A, Horowitz JF, Endert E \& Wolfe RR. (1993). Regulation of endogenous fat and carbohydrate metabolism in relation to exercise intensity and duration. Am J Physiol 265, E380-391.

Russell AP, Hesselink MK, Lo SK \& Schrauwen P. (2005). Regulation of metabolic transcriptional co-activators and transcription factors with acute exercise. Faseb j 19, 986-988.

Sacchetti M, Saltin B, Osada T \& van Hall G. (2002). Intramuscular fatty acid metabolism in contracting and non-contracting human skeletal muscle. The Journal of physiology 540, 387-395.

Schrauwen-Hinderling VB, Schrauwen P, Hesselink MK, van Engelshoven JM, Nicolay K, Saris WH, Kessels AG \& Kooi ME. (2003a). The increase in intramyocellular lipid content is a very early response to training. The Journal of clinical endocrinology and metabolism 88, 1610-1616.

Schrauwen-Hinderling VB, van Loon LJ, Koopman R, Nicolay K, Saris WH \& Kooi ME. 
(2003b). Intramyocellular lipid content is increased after exercise in nonexercising human skeletal muscle. Journal of applied physiology 95, 2328-2332.

Shepherd SO, Cocks M, Tipton KD, Ranasinghe AM, Barker TA, Burniston JG, Wagenmakers AJ \& Shaw CS. (2013). Sprint interval and traditional endurance training increase net intramuscular triglyceride breakdown and expression of perilipin 2 and 5 . The Journal of physiology 591, 657-675.

Shepherd SO, Strauss JA, Wang Q, Dube JJ, Goodpaster B, Mashek DG \& Chow LS. (2017). Training alters the distribution of perilipin proteins in muscle following acute free fatty acid exposure. The Journal of physiology 595, 5587-5601.

Siri WE. (1956). The gross composition of the body. Advances in biological and medical physics 4, 239-280.

Stellingwerff T, Boon H, Jonkers RA, Senden JM, Spriet LL, Koopman R \& van Loon LJ. (2007). Significant intramyocellular lipid use during prolonged cycling in endurance-trained males as assessed by three different methodologies. Am J Physiol Endocrinol Metab 292, E1715-1723.

Unger RH, Clark GO, Scherer PE \& Orci L. (2010). Lipid homeostasis, lipotoxicity and the metabolic syndrome. Biochim Biophys Acta 1801, 209-214.

van Loon LJ, Koopman R, Stegen JH, Wagenmakers AJ, Keizer HA \& Saris WH. (2003). Intramyocellular lipids form an important substrate source during moderate intensity exercise in endurance-trained males in a fasted state. The Journal of physiology 553, 611-625.

Vermathen P, Saillen P, Boss A, Zehnder M \& Boesch C. (2012). Skeletal muscle (1)H MRSI before and after prolonged exercise. I. muscle specific depletion of intramyocellular lipids. Magnetic resonance in medicine : official journal of the Society of Magnetic Resonance in Medicine / Society of Magnetic Resonance in Medicine 68, 1357-1367.

Wang H, Bell M, Sreenivasan U, Hu H, Liu J, Dalen K, Londos C, Yamaguchi T, Rizzo MA, Coleman R, Gong D, Brasaemle D \& Sztalryd C. (2011). Unique regulation of adipose triglyceride lipase (ATGL) by perilipin 5, a lipid droplet-associated protein. J Biol Chem 286, 15707-15715.

Whytock KL, Shepherd SO, Wagenmakers AJM \& Strauss JA. (2018). Hormone-sensitive lipase preferentially redistributes to lipid droplets associated with perilipin- 5 in human skeletal muscle during moderate-intensity exercise. The Journal of physiology 596, 2077-2090. 


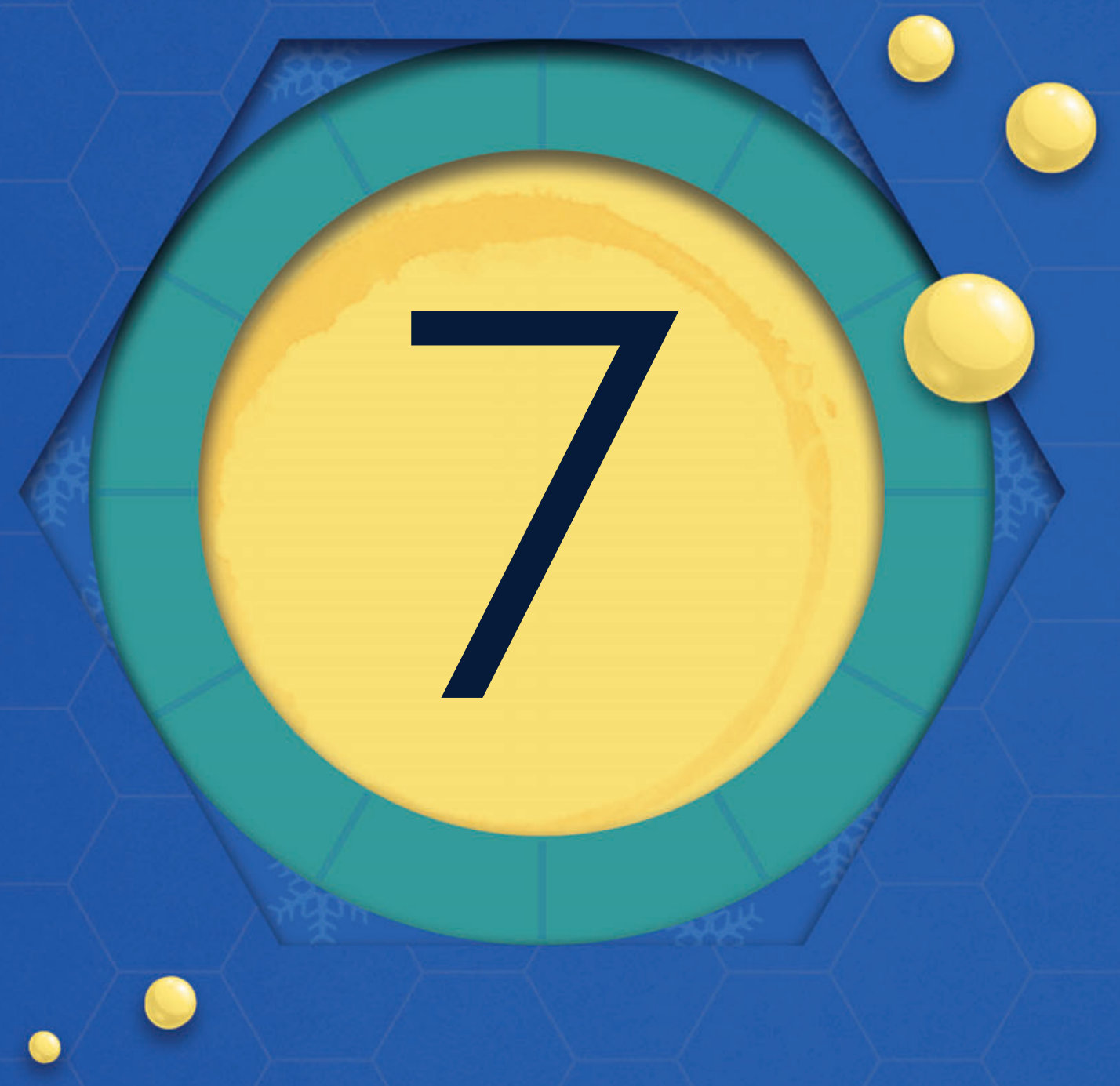




\section{A novel microscopy tool to study lipid droplet dynamics in vitro in human primary myotubes}

Nynke van Polanen, Anne Gemmink, Gert Schaart, Kèvin Knoops,

Patrick Schrauwen, Matthijs K.C. Hesselink

In preparation 


\section{Chapter 7}

\section{Abstract}

Intramyocellular lipids are stored as lipid droplets (LDs). LDs adjust to changes in supply and energy demand and this capacity is referred as LD dynamics. Compromised LD dynamics may contribute to metabolic disturbances observed in muscle insulin resistance. Many studies have investigated factors of LD dynamics, albeit in a static state. Therefore, a tool to study LD dynamics in a time-bound fashion is warranted. Thus, we aimed to develop an in vitro model to study LD dynamics in cultured human primary myotubes (HPMs) and the pioneering steps are described in this chapter. HPMs were first incubated with Bodipy-FL (green)-labelled C12 fatty acids (FAs) along with oleate (total lipid load of $50 \mu \mathrm{M}$ ) to form the first LDs within 12-24 hours. After washing, the HPMs were incubated with Bodipy-558/568 (red)-labelled C12 FAs together with oleate for another 6-12 hours. Retrospectively, LDs were classified as preformed LDs (formed during the first incubation without incorporating FAs from the second incubation), incorporating LDs (preformed LDs incorporating new FAs from the second incubation and therefore contain both fluorescent FAs) and new LDs (formed during the second incubation). Confocal and live-cell fluorescent microscopy were used to visualize LDs within HPMs. As expected, proof of concept results show that both fluorescent FAs were incorporated into LDs without the likelihood of false-positive or false-negative colocalization. The fluorescent FAs were heterogeneously formed within and between HPMs. The ratio of fluorescent FAs within a LD and the ratio of different LD pools can determine the dynamic nature of the individual LD and the total LD pool within a HPM respectively. Pilot data revealed a more rapid lipid accumulation in HPMs from insulin-sensitive donors compared to insulin-resistant donors. This tool can contribute to the mechanistic understanding of LD dynamics in donors with different metabolic phenotypes. 


\section{Introduction}

Lipid droplets (LD) are organelles consisting of a lipid core surrounded by a phospholipid monolayer (Farese \& Walther, 2009), present in virtually all cell types. LDs are essential storage sites of neutral lipids, such as triacylglycerols (TAGs) and cholesterol esters that can deliver fatty acids (FAs) for oxidative metabolism. Especially in skeletal muscle, which is subject to profound changes in energy demand and supply, LDs can readily release FAs for oxidation or take up FAs from the circulation and the sarcoplasm to avoid lipotoxicity to occur (Walther et al., 2017). The inherent capacity of LDs to readily adjust to changes in energy demand and supply is referred to as LD dynamics.

Compromised LD dynamics has been suggested to underlie metabolic aberrations as observed in the insulin-resistant state (Badin et al., 2013; Daemen et al., 2018). Most of these suggestions are based upon evaluation of proteins, genes or metabolites involved in LD dynamics, albeit under static conditions. Some studies have used stable isotope-based FA tracers to measure incorporation of exogenous FAs in the myocellular LDs and release of FAs to fuel fat oxidation in vivo (van Loon et al., 2003; Bergman et al., 2018) providing a surrogate marker of LD dynamics. Although valuable, this method provides only little mechanistic insight into factors contributing to LD dynamics. Moreover, this method does not provide information on the fate of the lipids, whether FAs are incorporating into new or already existing LDs. Such information may be beneficial in understanding how excess FA supply can lead to lipotoxicity.

This stresses the need for tool development to measure LD dynamics in a time-bound fashion. Hence, we aimed to develop an in vitro model to examine LD dynamics and FA handling in cultured human primary myotubes (HPMs), by using two differently labelled FAs (green and red). First, we add Bodipy-FL (green)-labelled C12 FAs together with oleate to form LDs within HPMs for 12-24 hours. After washing, the HPMs are incubated with Bodipy-558/568 (red)-labelled C12 FAs together with oleate for another 6-12 hours. With this design, we can determine the fate of the FAs and 


\section{Chapter 7}

examine if excess FAs (from the second incubation) are incorporated preferentially into new LDs or are shuttled towards LDs that were already present upon the first incubation. Ultimately, we aim to apply this novel tool to study differences in LD dynamics in human primary muscle cells cultured from insulin-resistant versus insulin-sensitive donors. This chapter describes the pioneering and essential steps in setting up this novel tool.

\section{Material and methods}

\section{Human primary myotubes culture}

Human primary myotubes (HPMs) were cultured from previously obtained muscle biopsies from an untrained healthy individual, a trained individual (Vosselman et al., 2015) and a patient with type 2 diabetes (T2DM) (Brouwers et al., 2017). All donors were male. The Medical Ethic Committee of Maastricht University approved these studies in accordance with the Declaration of Helsinki. All participants provided written informed consent before participation. Participant characteristics of these donors are displayed in Table 1. The muscle biopsies were obtained from the $\mathrm{m}$. vastus lateralis according to the Bergstrom method (Bergstrom, 1975) and HPMs were isolated and purified as previously described (Sparks et al., 2011). HPMs from the untrained donor were used for proof-of-principle experiments in fixed cells, HPMs from the trained individual and the patient were used for live-cell imaging.

Table 1. Participant characteristics of the donors

\begin{tabular}{lccc}
\hline & Untrained & Trained & T2DM \\
\hline Age $(\mathrm{y})$ & 23 & 31 & 56 \\
Sex & male & male & male \\
$\mathrm{BMI}\left(\mathrm{kg} / \mathrm{m}^{2}\right)$ & 22.7 & 22.7 & 31.9 \\
$\operatorname{VO}{ }_{2} \max (\mathrm{ml} / \mathrm{min} / \mathrm{kg})$ & 42.7 & 56.0 & 26.2 \\
$\operatorname{GIR}(\mathrm{ml} / \mathrm{h})$ & 250 & 290 & 100 \\
\hline
\end{tabular}


Satellite cells from the untrained healthy donor were grown on \#1 coverslips and cultured in growth medium containing Dulbecco's Modified Eagle Medium (DMEM) low glucose (5.5 mM) medium (Gibco, Thermo Fisher Scientific, Landsmeer, the Netherlands) supplemented with $10 \%$ foetal bovine serum (Gibco), $0.5 \mathrm{mg} / \mathrm{ml}$ bovine serum albumin (Sigma-Aldrich, Zwijndrecht, the Netherlands), $1 \mu \mathrm{M}$ dexamethasone (Sigma-Aldrich), $0.5 \mathrm{mg} / \mathrm{ml}$ fetuin (Sigma-Aldrich), $0.5 \mu \mathrm{g} / \mathrm{ml}$ Fungizone (Gibco), $50 \mu \mathrm{g} / \mathrm{ml}$ Gentamycin (Gibco), $10 \mathrm{ng} / \mathrm{ml}$ human epidermal growth factor (Gibco) and $0.5 \%$ penicillin and streptomycin (Gibco). At $90 \%$ confluence, differentiation was triggered by replacing the culture medium with alpha Minimum Essential Medium ( $\alpha$-MEM) low glucose medium (Gibco) supplemented with $2 \%$ foetal bovine serum, $0.5 \mathrm{mg} / \mathrm{ml}$ fetuin, and $2 \%$ penicillin and streptomycin (Gibco), for approximately 6 days. Medium was replaced every $2-3$ days and $\mathrm{HPMs}$ were kept at $37^{\circ} \mathrm{C}$ and $5 \% \mathrm{CO}_{2}$.

\section{Double labelled experiment with two fluorescent fatty acids}

To study LD dynamics in HPMs a double labelled experiment was designed with two different FA labels, inspired by work from others in hepatocytes (Wang et al., 2010) and mouse embryonic fibroblasts (Rambold et al., 2015). As depicted in Figure 1A, we used Bodipy FL-C12 (D3822; Invitrogen, Thermo Fisher Scientific, Landsmeer, the Netherlands) and Bodipy 558/568-C12 (D3835; Invitrogen), which yield a green and red fluorescent signal upon excitation respectively. Both FAs have a chain of 12 carbon atoms with a Bodipy label attached to the methyl end (Figure 1A). In vitro, these fluorescent FAs act similar as an 18-carbon FA (Rambold et al., 2015; Kolahi et al., 2016), such as oleate.

In general, the experiment started after 5 days of differentiation and HPMs were incubated for 12-24 hours with Bodipy FL-C12 along with unlabelled oleate (total lipid load of $50 \mu \mathrm{M})$. After washing with Dulbecco's Phosphate-Buffered Saline (DPBS; Gibco), Bodipy 558/568-C12 was added in combination with oleate for 6-12 hours, hence resulting in a similar lipid load of $50 \mu \mathrm{M}$. In this way, we classify LDs present upon first incubation as preformed LD as these are formed before the second incubation, reflecting an initial reservoir of LDs before an additional supply 


\section{Chapter 7}

of FAs. After the second incubation we differentiate between LDs formed during the second incubation (new LDs) and preformed LDs incorporating newly supplied FAs (incorporating LDs) (Figure 1B). Preformed LDs that do not take up newly supplied FAs (or barely) remain classified as preformed LDs. Others have used different concentration of Bodipy FL-C12 and Bodipy 558/568-C12 in various cell types, ranging from $1 \mu \mathrm{M}$ - 6 M (Wang et al., 2010; Rambold et al., 2015; Kolahi et al., 2016). To our knowledge, we are the first to supply these fluorescent FAs to HPMs. Therefore, we have tried different concentrations $(1,2,3,4,6,8$, and $16 \mu \mathrm{M})$ and we found an optimal concentration of $3 \mu \mathrm{M}$, to obtain minimal background staining and an optimal fluorescence intensity. To facilitate FA uptake by the HPMs, the fluorescent FAs were accommodated with unlabelled oleate. The concentration of oleate was matched with concentration of the fluorescent FAs to obtain a final lipid load of 50 $\mu \mathrm{M}$ in each experiment. Furthermore, we have tried different incubation time periods.

A

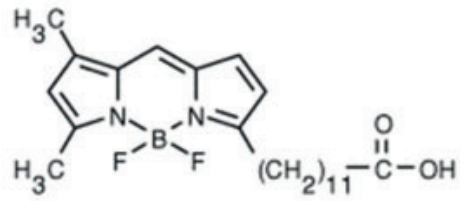

Bodipy FL-C12

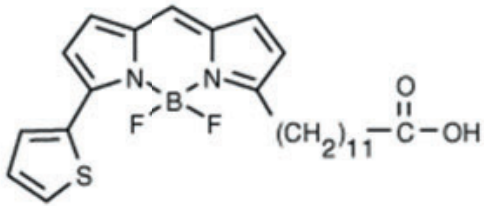

Bodipy 558/568-C12

B

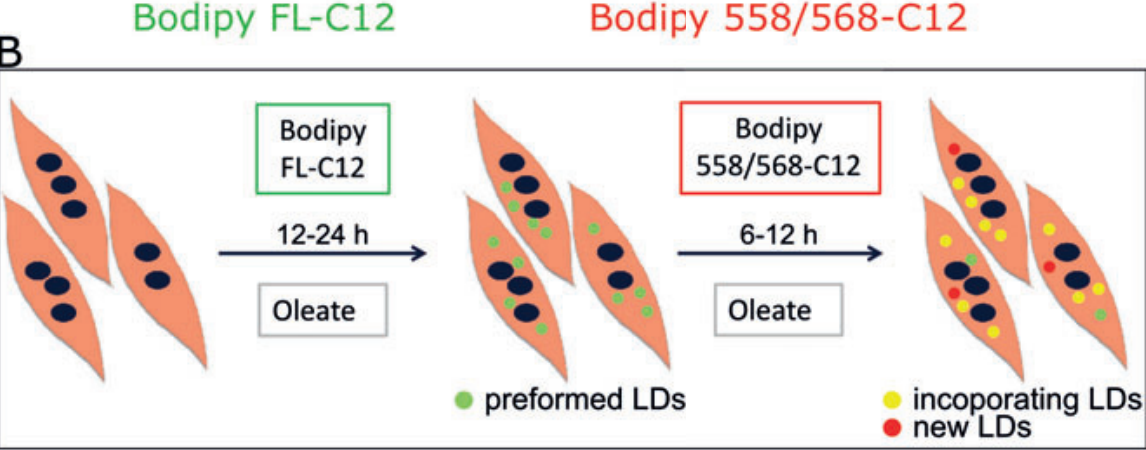

Figure 1. General experimental set-up. A) Chemical structure of Bodipy FL-C12 and Bodipy 558/568C12. Images are altered from the Thermo Fisher Scientific website. B) Differentiated human primary myotubes were incubated first with Bodipy-FL (green)-labelled C12 fatty acids (FAs) along with oleate for 12-24 hours, followed by an incubation with Bodipy-558/568 (red)-labelled C12 FAs along with oleate for another 6-12 hours. Every incubation had a total lipid load of $50 \mu \mathrm{M}$. Incubation with both fluorescent FAs may result in a variety of green, red and yellow (both fluorescent FAs present) lipid droplets. LDs can be formed during first incubation (preformed LDs, depicted here in green), LDs formed during the second incubation (newly formed LDs, depicted here in red) and preformed LDs incorporating newly supplied FAs (incorporating $L D s$, depicted here in yellow). 
The first incubation was between 12-24 hours, whereas the second incubation either 6 or 12 hours. To examine if the order of adding the different fluorescent FAs affected the incorporation of the label into the LD, we reversed the order of incubation with the fluorescent FAs in a parallel experiment (i.e., now starting with Bodipy 558/568C12 incubation, which after a thorough rinse, was followed by an incubation with Bodipy FL-C12).

\section{Fixation and imaging of human primary myotubes}

After incubation with the FAs, the cells were fixed with 3.7\% formaldehyde and stained with DAPI to visualize nuclei (4',6-diamidino-2-phenylindole, dihydrochloride; D1306; Invitrogen; 1:100). Coverslips were mounted on object glass slides with Mowiol and imaging was performed with a Leica TCS SP8 confocal microscope using a 100x 1.4 N.A. oil immersion objective. The fluorophores DAPI, Bodipy FL-C12 and Bodipy 538/568-C12 were excited with a laser wavelength of 405, 500 and $558 \mathrm{~nm}$ respectively and emission fluorescence was detected between 415-460 nm, 510-530 nm and 568-630 $\mathrm{nm}$ respectively (Figure 2). All fluorophores were excited in sequence. Deconvolution of the confocal images was performed with Huygens Professional software (Scientific Volume Imaging B.V., Hilversum, the Netherlands).

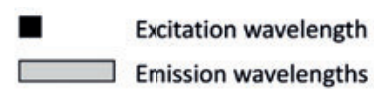

Channel 1 - DAPI /MDH (blue)

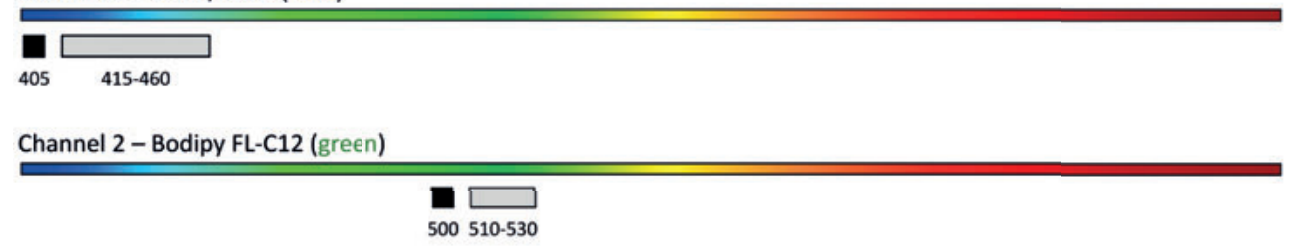

Channel 3-Bodipy 558/568-C12 (red)

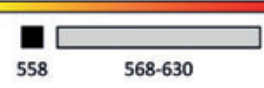

Figure 2. Settings of the excitation and emission wavelengths for DAPI/MDH, Bodipy FL-C12 and Bodipy 558/568-C12. 


\section{Chapter 7}

\section{Testing for crosstalk, bleed-through and Fluorescence Resonance Energy Transfer}

When applying two fluorophores within one assay, it is important to check for crosstalk, bleed-through or Fluorescence Resonance Energy Transfer (FRET), as these might bias the results. For this, the settings for excitation and emission were applied as depicted in Figure 2, with an additional excitation of $500 \mathrm{~nm}$ with an emission window of 568-630. It should be noted that a combination of an excitation wavelength of 558 together with an emission frame 510-530 is not applicable, as a stoke shift takes place in fluorescent microscopy. An overview of the experiment is depicted in Table 2. To test for crosstalk and bleed-through, HPMs were incubated with respectively solely Bodipy 558/568-C12 (16 $\mu \mathrm{M})$ and oleate $(34 \mu \mathrm{M})$ for 24 hours, or with solely Bodipy FL-C12 $(6 \mu \mathrm{M})$ and oleate $(44 \mu \mathrm{M})$ for 30 hours. HPMs were fixed, stained with DAPI and imaged as previously described.

Table 2. Overview of incubations and settings for crosstalk, bleed-through and FRET.

\begin{tabular}{lccccc}
\hline & \multicolumn{2}{c}{ Incubation } & \multicolumn{2}{c}{ Settings } \\
\hline & Bodipy label & $\begin{array}{c}\text { Concentration } \\
(\boldsymbol{\mu M})\end{array}$ & $\begin{array}{c}\text { Time } \\
(\mathbf{h})\end{array}$ & $\begin{array}{c}\text { Excitation and Emission } \\
(\mathbf{n m})\end{array}$ \\
\hline Crosstalk & Bodipy 558/568-C12 & 16 & 24 & 500 & $510-530$ \\
& & & & 500 & $568-630$ \\
Bleed- & Bodipy FL-C12 & 6 & 30 & 558 & $568-630$ \\
through & & & & 500 & $510-530$ \\
& & & & 500 & $568-630$ \\
FRET & Bodipy FL-C12 & 6 & 24 & 475 & $568-630$ \\
& Bodipy 558/568-C12 & 2 & 6 & 475 & $660-700$ \\
& & & & 558 & $660-700$ \\
\hline
\end{tabular}

FRET = Fluorescence Resonance Energy Transfer

As Bodipy FL-C12 and Bodipy 558/568-C12 can be both incorporated in the same LD and are therefore in close proximity, FRET can occur and lead to an overestimation of the red fluorescence and an underestimation of the green fluorescence. To test whether FRET occurred, HPMs were incubated with $6 \mu \mathrm{M}$ Bodipy FL-C12 $(+44 \mu \mathrm{M}$ 
oleate) for 24 hours and, after washing, incubated with $2 \mu \mathrm{M}$ Bodipy 558/568-C12 $(+48 \mu \mathrm{M}$ oleate) for 6 hours (Table 2$)$ and subsequently fixed, stained with DAPI and imaged. Alternative imaging settings were applied to make sure that there was no contamination of crossover or bleed-through. There is a known excitation and emission spectrum for Bodipy FL-C12 (Thermo Fisher), but not for Bodipy 558/568C12. Therefore, an emission spectrum was made for both fluorophores using confocal microscopy in the $\lambda$ xy settings. Bodipy FL-C12 was excited with $480 \mathrm{~nm}$ and images were taken with steps of 5 nm starting from 490 until 595 nm. Bodipy 558/568-C12 was excited with $500 \mathrm{~nm}$ and images were taken every $9 \mathrm{~nm}$ starting from 510 until 776. With this information possible FRET emission was measured between 660-700 when excited with 475 (Table 2).

\section{Protocol for MDH staining in fixed human primary myotubes}

In our experiments, the fluorescent FAs are incubated together with non-labelled FAs (oleate). There is a possibility that LDs are formed with unlabelled oleate, but without any of the Bodipy-labelled FAs, and are therefore unintentionally missed in our observations. Thus, we used the blue fluorescent neutral lipid dye MDH (SM1000a; Abgent, San Diego, USA; 1:250) as a control to stain all LDs after incubation with Bodipy FL-C12 and Bodipy 558/568-C12. In this experiment, HPMs from an untrained donor were incubated overnight with $6 \mu \mathrm{M}$ Bodipy FL-C12 (+ $44 \mu \mathrm{M}$ oleate) followed by a 6-hour incubation with $2 \mu \mathrm{M}$ Bodipy 558/568-C12 (+ $48 \mu \mathrm{M}$ oleate). In parallel, the order of the fluorescent FAs was reversed. After incubation with both FAs, the HPMs were fixed with 3.7\% formaldehyde and stained with $\mathrm{MDH}$, to visualize all neutral lipids in a Bodipy-labelled FA independent manner. Again, coverslips were mounted on object glass slides and imaging was performed with a Leica TCS SP8 confocal microscope. MDH was excited with a laser wavelength of 405 and emission fluorescence was detected between 415-460 nm (Figure 2).

Every image contains three channels: the $\mathrm{MDH}$ channel with yields a blue fluorescence upon excitation (blue channel), the Bodipy FL-C12 channel that yields green fluorescence (green channel) and the Bodipy 558/568-C12 channel that yields 


\section{Chapter 7}

red fluorescence (red channel). The first quick step to determine colocalization, is to visually examine the merged images for blue LDs. These blue MDH-positive LDs are an indication of LDs containing only lipids with oleate incorporation, but in the absence of Bodipy-labelled FAs. Furthermore, a Pearson correlation coefficient (PCC) was calculated. For this, the total number of LDs were calculated in each image. For every LD, the mean intensity of each fluorophore (MDH, Bodipy FL-C12 or Bodipy $558 / 568-C 12$ ) was calculated and the $r$ is computed based on these mean intensity values. A min-max normalization is performed to plot the normalized mean intensity of each fluorophore within each LD (where each data point represents a LD). In this way, MDH (blue channel) is correlated with Bodipy FL-C12 (green channel), Bodipy 558/568-C12 (red channel), and with Bodipy FL-C12 and Bodipy 558/568-C12 combined. Among LDs, different concentrations of Bodipy 558/568-C12 or Bodipy FL-C12 can be present. We are primary interested if a LD contains MDH with at least one of the fluorophores and not solely MDH (false-negatives). In this way a Manders Colocalization Coefficients, M1 and M2, is more feasible than PCC, because PCC is influenced by the ratio of fluorescent intensities (Dunn et al., 2011). As this ratio most likely varies within each LD, this might cause a low PCC, while they do colocalize. Manders Colocalization Coefficients compare the pixels from two channels and provides two values: the fraction of channel A in channel B (M1) and fraction B in channel A (M2). Therefore, M1 and M2 were calculated in addition to PCC.

\section{Quantification of fixed human primary myotubes}

Furthermore, we wanted to test our customized script made in the Image J software (NIH, Bethesda, USA) (Schneider et al., 2012) to quantify different LD populations (preformed, incorporating and new LDs) originated from the double labelled experiment. HPMs were first incubated for 12 hours with $3 \mu \mathrm{M}$ Bodipy FL-C12 (+ $47 \mu \mathrm{M}$ oleate), followed by a 12-hour incubation with $3 \mu \mathrm{M}$ Bodipy 558/568-C12 (+ $47 \mu \mathrm{M}$ oleate). In addition, we reversed the order of incubation of the labels in a parallel experiment. After incubation, HPMs were fixed, stained with DAPI and imaged according to the settings described in Figure 2. Images were deconvoluted 
before quantification and quantification was performed on single images (no stacks). The quantification steps in the customized Image J script are described below, where Bodipy FL-C12 was used for the first incubation step.

In the ImageJ script, first a binary image is made from the green channel (Bodipy FL-C12) and the red channel (Bodipy 558/568-C12). An image of all LDs is computed by adding the binary image of the green channel to the red channel.

All LDs = binary image green channel + binary image red channel

Determination of preformed LDs, incorporating LDs and new LDs requires multiple steps. To determine new LDs, the binary image of the green channel is subtracted from the binary image with all LDs. This new created image will contain newly formed LDs, but also the incorporation of new FAs into LDs that were present already (the incorporating LDs). By using a white top hat filter, the incorporating LDs will be omitted from the image with only the newly formed LDs remaining in the image.

New LDs = all LDs - binary image green channel

$\rightarrow$ white top hat filter

To define incorporating LDs, the binary image of the green channel is again subtracted from the binary image with all LDs. Next, the image of the new LD is subtracted. On the resulting image, filtering is applied based on size $\left(0.05 \mu \mathrm{m}^{2}\right.$-infinity) and shape (circularity 0-0.95), to remove single pixel remnants. This image is compared to the image containing all LDs. LDs that have signal in both images are classified as incorporating LDs.

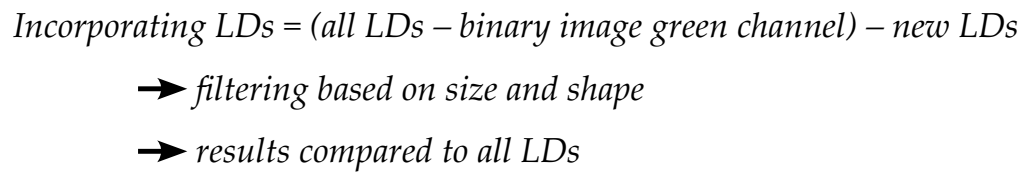

To identify the preformed LDs, the binary images of new LDs and incorporating LDs are combined and compared to the binary image of all LDs. LDs that are present in the image of all LDs (determined by particle analysis), but not in the combined image of new LDs + incorporating LDs are considered preformed LDs. 


\section{Chapter 7}

Preformed $L D s=$ all LDs $-($ new LDs + incorporating $L D s)$

$\rightarrow$ determined by particle analysis

From the images with the LD classes (preformed, incorporating and new LDs) a segmentation image can be computed to visualize the different groups and compare it with the original image. Furthermore, each LD is determined as a region of interest (ROI) and within this ROI the mean intensities of Bodipy FL-C12 and Bodipy 558/568C12 are determined from the raw image (non-deconvoluted).

In this way we can determine the mean intensity of each fluorescent FA per LD. These values can be used to make scatter plots and to determine the intensity ratio of the two Bodipy labelled FAs. If a LD has a higher new/old FA ratio, the LD is more dynamic than a LD with a low ratio. Moreover, a ratio of newly formed LDs/ preformed LDs can be computed, whereas a high ratio means a higher fraction of new LDs within this image.

\section{Fluorescent live-cell microscopy}

Live-cell imaging was performed to study lipid incorporation over time and to get more information about the ideal incubation time. For live-cell imaging, we used HPMs from a trained individual and a patient with T2DM. The experiment was performed twice. The first experiment was performed with HPMs from a trained individual, the second experiment with HPMs from both trained and T2DM individuals. All experiments were performed in duplicate (wells) and within each well, images were taken on four different locations. Cell culturing was similar as for the untrained individual, aside from the myoblasts were grown and differentiated on a $\mu$-Slide 8 Well (80826; Ibidi, Germany). After 6 days of differentiation, the differentiation medium was replaced by phenol red-free medium supplemented with $5.5 \mathrm{mM}$ glucose, $2 \mathrm{mM}$ glutamine (GlutaMAX, Gibco) and 2\% FBS. HPMs were prior imaging incubated with CellMask (C10046; Invitrogen; 1:1000) for 30 minutes at $37^{\circ} \mathrm{C}$ to better visualize the cells. After washing with DPBS, cells were incubated with oleate (50 $\mu \mathrm{M}$ ) and Bodipy 493/503 (D3922; Molecular Probes, Leiden, 
the Netherlands; $1: 500$ ) in phenol red-free medium at $\mathrm{t}=0$ and imaged with a FEI CorrSight spinning disk confocal microscope, equipped with an ORCA-Flash 4.0 V2 CMOS camera. Imaging was performed with a 40x 0.9 N.A. air objective (Zeiss, Breda, the Netherlands) at $37^{\circ} \mathrm{C}\left(5 \% \mathrm{CO}_{2}\right)$ for 24 hours with 1-hour intervals. Total LD area (Bodipy signal, $\mu \mathrm{m}^{2}$ ), total LD number (count) and average LD size (Total LD area/total LD number) was determined in each image. To normalize for differences in surface covered by HPMs in the field of view, fold changes were calculated by dividing each measurement by the baseline measurement $(t=1)$. Before incubation, no lipids were added to the medium.

\section{Results}

\section{Crosstalk and bleed-through can be avoided and Fluorescence Resonance Energy Transfer is negligible}

To avoid bias in our results, we have tested for crosstalk, bleed-through and FRET. Crosstalk (Figure 3A) takes place when another fluorophore is excited along with the desired fluorophore at a certain wavelength. In our case, if crosstalk occurs, the excitation wavelength used for Bodipy FL-C12 excites Bodipy 558/568-C12 as well. Therefore, we tested crosstalk with solely Bodipy 558/568-C12 along with the excitation settings of Bodipy FL-C12 and collecting in the emission window of Bodipy 558/568-C12. Bleed-through (Figure 3B), however, occurs when one fluorophore is excited and its fluorescence signal can be detected in both emissions windows. For example, when Bodipy FL-C12 is excited with $500 \mathrm{~nm}$, fluorescence is not only collected between 510-530, but also between 568-630. Therefore, bleed-through was tested in presence of Bodipy FL-C12 solely. Bodipy FL-C12 was excited with $500 \mathrm{~nm}$ and emission was collected between 568-630.

When Bodipy 558/568-C12 was excited with 500, we could not detect fluorescence between 510-530. Between 568-630, we detected a low fluorescence signal, indicating that Bodipy 558/568-C12 can be excited with $500 \mathrm{~nm}$ (Figure 3C). However, this signal (grey line) was only $8 \%$ of the signal when Bodipy 558/568-C12 was excited by 558 


\section{Chapter 7}

nm (red line). Bodipy FL-C12 could not be excited with 558, as no signal was present (red line, Figure 3D). To test for bleed-through, Bodipy FL-C12 was excited with 500 and fluorescence was examined between 510-530 and 568-630. Bodipy FL-C12 can be excited with 500 and fluorescence is detected in the desired emission frame, but also some signal is present between 568-630 (grey line, Figure 3D), indicating bleedthrough. The bleed-through fluorescence is $23 \%$ of the fluorescence detected between 510-530 (green line, Figure 3D). Compared to crosstalk, the bias by bleed-through is a

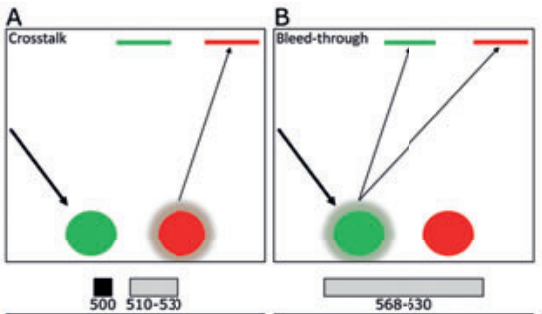

C
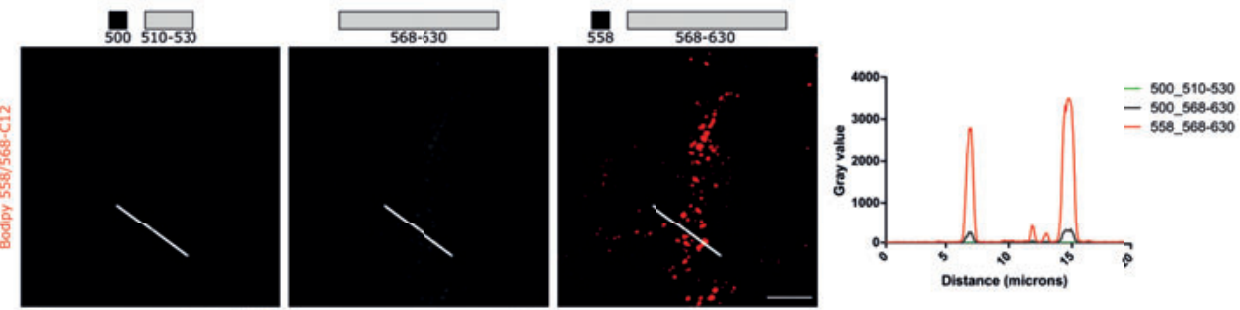

D
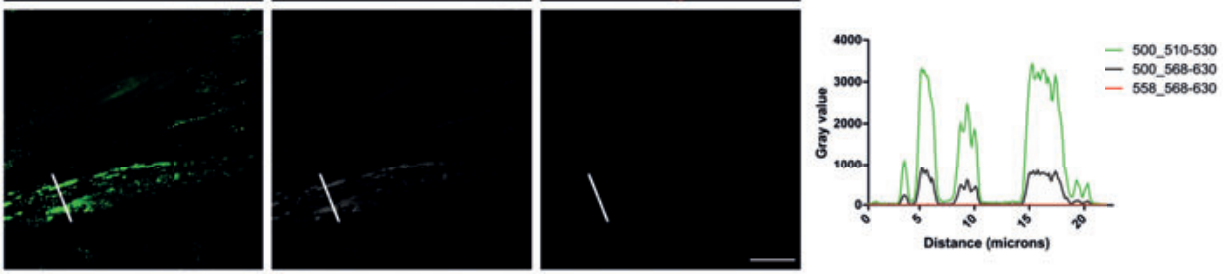

E
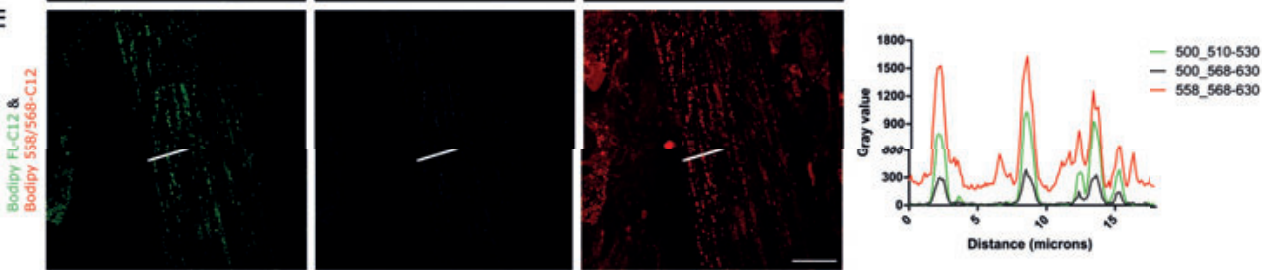

Figure 3. Crosstalk and bleed-through analysis with Bodipy FL-C12 and Bodipy 558/568-C12. A) Schematic overview of crosstalk. The sample is excited with $500 \mathrm{~nm}$ (to initially excite the green fluorophore). If crosstalk would take place, the red fluorophore would be excited as well and send fluorescence. B) Schematic overview of bleed-through. If the green fluorophore is excited with $500 \mathrm{~nm}$, fluorescence can be measured in both 510-530 and 568-630 if bleed-through would take place. C-E) Images taken on the confocal microscope from fixed cells incubated with solely Bodipy 558/568-C12 (16 $\mu \mathrm{M})$ and oleate (34 $\mu M)(C)$, or with solely Bodipy FL-C12 $(6 \mu M)$ and oleate $(44 \mu M)(D)$ or incubated with both Bodipy FLC12 $(6 \mu \mathrm{M})$ and Bodipy 558/568-C12 $(2 \mu \mathrm{M})$ supplemented with oleate (44 and $48 \mu \mathrm{M}$ respectively) (E). Sections were excited with 500 and 558 with an emission window of 510-530 and 568-630. The graphs represent the grey values of each picture, measured among the white line with a certain distance $(\mu \mathrm{m})$. Scale bar $=10 \mu \mathrm{m}(C)$ or $20 \mu \mathrm{m}$ (D\&E). 
bit stronger. It should be noted, however, that both cross-talk (8\%) and bleed through $(23 \%)$ is still way lower than the fluorescent signal of interest. When both Bodipylabelled FAs were present (Figure 3E), both Bodipy Fl-C12 and Bodipy 558/568-C12 were indeed detectable within the corresponding emission windows. Fluorescence was detected between 568-630 when the sample was excited with 500 (grey line). This signal is probably a combination of crosstalk from Bodipy 558/568-C12 and (based on the previous results) predominantly bleed-through from Bodipy FL-C12. However, it was on average 39\% of the fluorescence detected between 510-530 (green line) and 14\% of the fluorescence between 568-630 (red line). Based on these results, is important to excite the Bodipy-labelled FAs sequentially rather than simultaneously. Since Bodipy 558/568-C12 cannot emit light between 510-530 (Figure 3C) and Bodipy FL-C12 cannot be excited with 558 (Figure 3D), crosstalk and bleed-through can be avoided and the likelihood of bias by false-positive colocalization is negligible.

In addition, we examined potential bias originating from FRET, which means the capacity to transfer energy from one fluorophore (donor) to another when in close proximity and thereby excite that fluorophore (acceptor). This can result in an overestimation of the fluorescence of acceptor fluorophore and an underestimation of the donor fluorophore. As green fluorescence contains more energy, FRET can only take place from Bodipy FL-C12 to Bodipy 558/568-C12 and not reversely (Figure 4A). Ideally, FRET would be measured by using an excitation of $500 \mathrm{~nm}$ (excitation Bodipy FL-C12) and collecting emission between 568-630 (emission Bodipy 558/568C12). However, the emission collected between 568-630 would be contaminated with crosstalk and bleed-through as shown in Figure 3. To find settings in which we still can measure FRET, but to exclude contamination by crosstalk and bleed-through, an emission spectrum of each fluorophore was determined (Figure 4B). From these results we observed that Bodipy FL-C12 could not be detected in an emission window of 660-700 (supplementary Figure S1A), but Bodipy 558/568-C12 was still detectable (red line, Figure 4C). Bodipy FL-C12 was excited with 475 (which cannot excite Bodipy 558/568-C12, see Figure S1B) and possible FRET would have been detected between 660-700. As shown in Figure 4C, no fluorescence was detected (grey line), 


\section{Chapter 7}

indicating that FRET is negligible. As a control, green fluorescence was measured between 510-530, indicated that Bodipy FL-C12 can be excited with $475 \mathrm{~nm}$ (green line, Figure 4C).
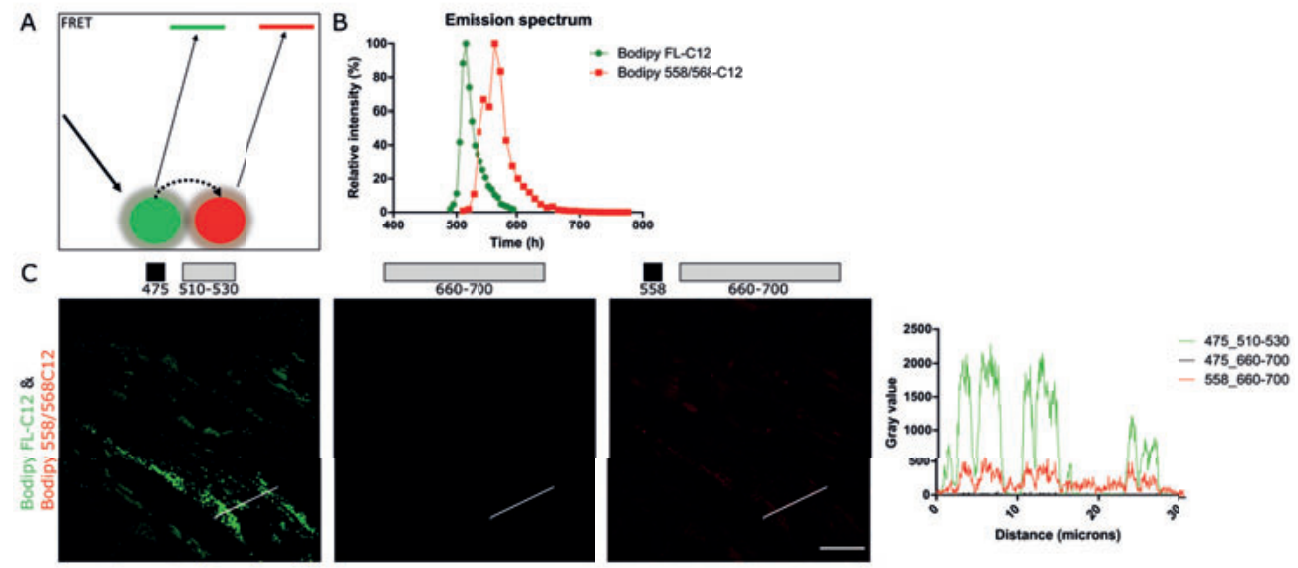

Figure 4. Analysis of fluorescence resonance energy transfer with Bodipy FL-C12 and Bodipy 558/568-C12. A) Schematic overview of Fluorescence Resonance Energy Transfer (FRET). When the sample is excited with $500 \mathrm{~nm}$ (to initially excite the green fluorophore), FRET can take place when both fluorophores are nearby. In that case, the green fluorophore can transfer energy to the red fluorophore which emits subsequently fluorescence. B) Emission spectrum of Bodipy FL-C12 and Bodipy 558/568-C12 analysed with confocal microscopy. C) Images taken on the confocal microscope from fixed cells incubated with Bodipy FL-C12 (6 $\mu \mathrm{M})$ and Bodipy 558/568-C12 (2 $\mu \mathrm{M})$ supplemented with oleate (44 and $48 \mu \mathrm{M}$ respectively). Sections were either excited at 475 or $558 \mathrm{~nm}$, with an emission of 510-530 or 660-700. These settings are chosen to make sure that the red fluorophore is not excited with $475 \mathrm{~nm}$ and the green fluorescence cannot be detected in the 660-700 emission window. If FRET would take place, it would be detected in the second image (475_660-700), however no fluorescence is observed and the quantified grey values are negligible. Grey values are measured among the white line (displayed in the images) and visualized in the corresponding graph. Scale bar $=20 \mu \mathrm{m}$. 


\section{Bodipy FL-C12 and Bodipy 558/568-C12 are incorporated into all lipid droplets present}

We were interested if our experiment (incubation of both fluorescent FAs) resulted in LDs with one or both fluorescently labelled FAs and not in LDs with solely oleate. Thus, if we image Bodipy FL-C12 and Bodipy 558/568-C12, do we include all LDs? To test this, HPMs were fixed after incubation with both fluorescent labelled FAs and stained with MDH to visualize all neutral lipids. Upon successive incubation of Bodipy FL-C12 and subsequently Bodipy 558/568-C12 (or reversely) (Figure 5A and 5B) in combination with oleate, none of the LDs detected stained entirely (and solely) blue upon MDH. This indicates that all LDs within the cells incorporated at least some of the exogenous Bodipy-labelled FAs, irrespective of the order of fluorescent FAs.

Determining colocalization by simply observing merged images may be subjective, therefore from a cropped image (Figure 5C) scatter plots were computed from the normalized mean intensity of each fluorophore present in individual LDs (Figure 5D). Interestingly, we plotted the (normalized) mean intensities of Bodipy FL-C12 and Bodipy 558/568-C12 from each LD and observed two clear distinctive pools of LDs (Figure 5D). One group of LDs contained relative more Bodipy FL-C12 (here: new LDs), while the other group mainly Bodipy 558/568-C12 (here: preformed LDs). To quantify whether using our experimental set-up resulted in labelling of all LDs present, we performed a Pearson's correlation of MDH (blue channel) with Bodipy FL-C12 (green channel) and Bodipy 558/568-C12 (red channel) combined (i.e. yellow channel in Figure 5). This resulted in a PCC of $r=0.530$ and very few LDs with solely MDH signal were detected. PCC is generally influenced by intensities of two fluorophores or the proportion of the fluorophores within a LD. This may cause a low value despite colocalization. To circumvent this influence and consider co-occurrence, Manders Colocalization Coefficients M1 and M2 were calculated to see if MDH positive LDs also contained Bodipy labelled FAs (M1) and if a LD with Bodipy labelled FAs also stains positive with MDH (M2). The results for the images depicted in Figure 5C are shown in Table 3. These results show that the fraction in the 


\section{Chapter 7}

MDH channel is also present in the green and red channel combined (yellow: blue; $\mathrm{M} 2=1$ ), indicating that our experimental set-up results in labelling of all LDs present.

Table 3. Manders Colocalization Coefficients

\begin{tabular}{lcc}
\hline Channel A : Channel B & M1 & M2 \\
\hline Red : Blue & 0.998 & 0.979 \\
Green : Blue & 0.976 & 1.000 \\
Red : Green & 1.000 & 0.960 \\
Yellow : Blue & 0.994 & 1.000 \\
\hline
\end{tabular}

M1: the fraction of probe $A$ in areas containing probe $B ; M 2$ : the fraction of probe $B$ in areas containing probe A; Channel red = Bodipy 558/568-C12; Channel blue = MDH; Channel green = Bodipy FL-C12; Channel yellow $=$ Bodipy 558/568-C12 + Bodipy FL-C12

Upon making these computations in a total of 20 images, the average M1 and M2 values (yellow:blue) were both 0.99 , indicating a perfect colocalization (close to 1 ), irrespective of the order of fluorescent FAs. For Bodipy FL-C12, the average M1 and M2 values were 0.99 and 0.86 respectively (green:blue). For Bodipy 558/568-C12, the average M1 and M2 of these 20 images was 0.99 and 0.95 respectively (red:blue). These values imply that the presence of solely blue LDs (i.e. LDs with oleate but without fluorescently labelled FAs) is negligible. 


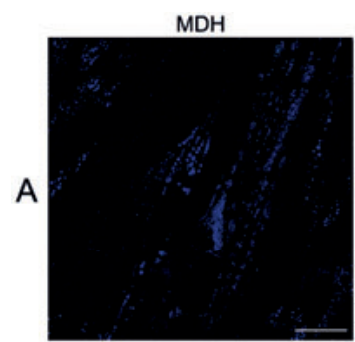

$\mathrm{MDH}$
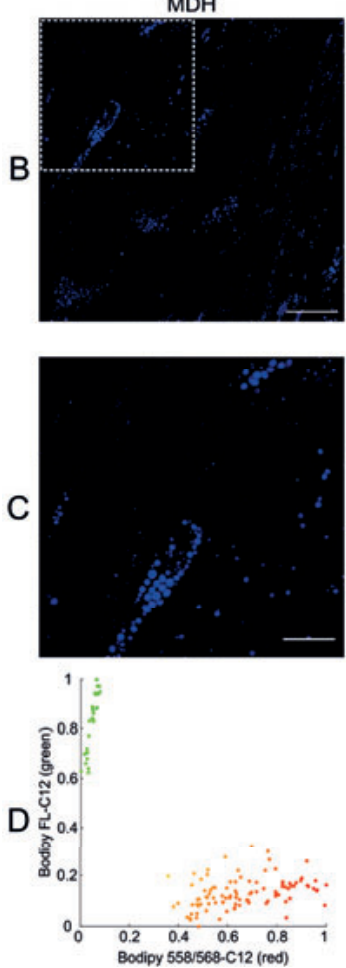

Bodipy FL-C12

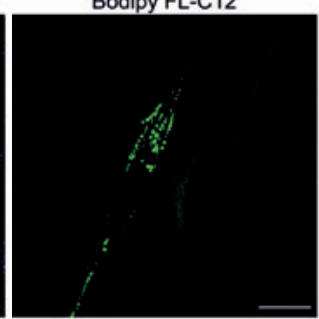

Bodipy 558/568-C12
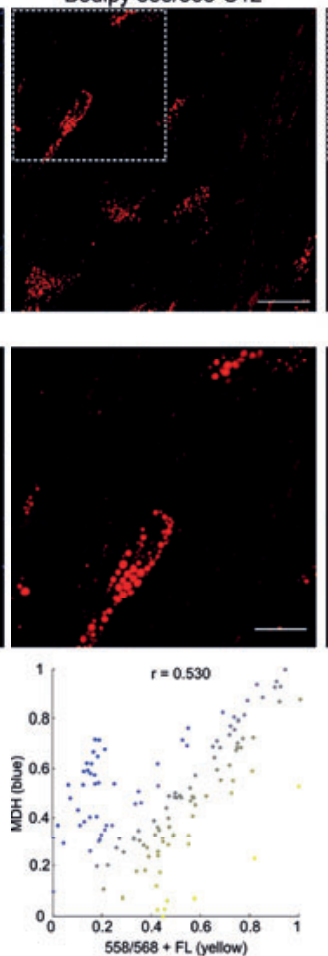

Bodipy 558/568-C12

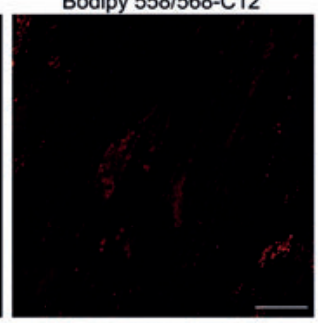

Bodipy FL-C12
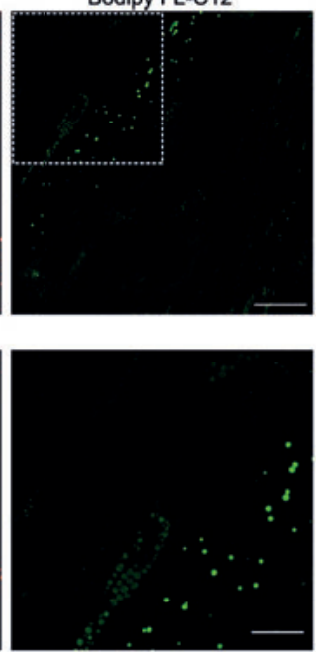

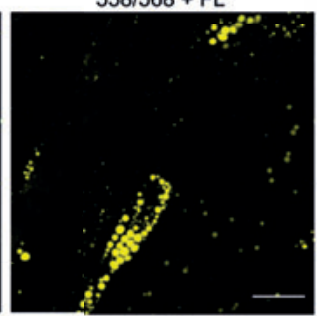

Merged

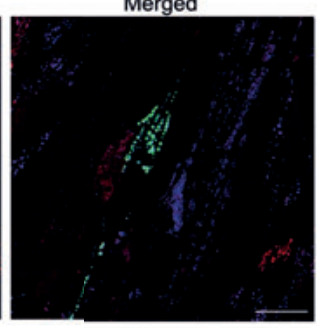

Merged

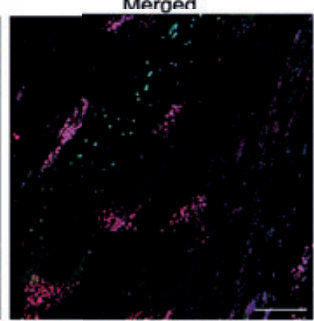

$558 / 568+\mathrm{FL}$

Figure 5. Fluorescent fatty acids are incorporated into all lipid droplets. Differentiated primary muscle cells from an untrained individual after an overnight incubation with $6 \mu \mathrm{M}$ Bodipy FL-C12 followed by a 6-hour incubation with $2 \mu \mathrm{M}$ Bodipy 558/568-C12 (A) or the other way around (B). Cells are fixed with $3.7 \%$ formaldehyde, all neutral lipids are stained with $\mathrm{MDH}$ (blue) and sections are imaged with a laser scanning confocal microscope. Scale bar $=20 \mu \mathrm{m}$. C) Cropped images from panel B. Last image is the result of the addition of the red image (Bodipy 558/568-C12) and the green image (Bodipy FL-C12). Scale bar $=10 \mu \mathrm{m}$. D) Scatter plots of normalized (min-max normalization) mean intensity values within each lipid droplet (LD). Each dot represents a LD. Normalized mean intensity of Bodipy 558/568-C12 (red) within each $L D$ is plotted against the normalized mean intensity of Bodipy FL-C12 (green) (graph 1). This is repeated for Bodipy 558/568-C12 + Bodipy FL-C12 (yellow) vs MDH (graph 2). For the second graph, a Pearson correlation coefficient $(r)$ is calculated. 


\section{Chapter 7}

\section{Time course analysis to determine the incubation time}

Previously, different incubation times have been used for the fluorescent FAs in different cell cultures (Wang et al., 2010; Rambold et al., 2015). For our HPMs, we wanted to determine how long it takes to incorporate the lipids into the LDs from the medium and therefore which timeframes would be most appropriate for the first and second lipid incubations. To test this, we used live-cell imaging and HPMs were incubated with Bodipy 493/503 together with $50 \mu \mathrm{M}$ oleate. After incubation, the cells were immediately followed for 24 hours and an image was taken hourly.

After addition of FAs, intracellular lipid content increased and reached a plateau after approximately 10 hours (Figure 6). Both LD area and LD number did not evidently change after this time point. The increase in lipid area is largely explained by an increase in LD number. LD size increased slightly over time, but would not noticeably influence lipid area. In Figure 6, the first experiment is displayed as Trained-1 (Figure 6A-C, including duplicates), the second experiment is labelled as Trained-2 (Figure 6D-F, including duplicates) and mean values of both experiments are displayed in Figure 6G-I. In the first experiment, the plateau is observed a bit earlier in time compared to the second experiment (Figure 6A vs 6D). This difference may originate from a higher absolute baseline lipid area in experiment 1 compared to experiment $2\left(678.45 \pm 323.04 \mu \mathrm{m}^{2}\right.$ vs $169.41 \pm 62.55 \mu \mathrm{m}^{2}$; mean $\left.\pm \mathrm{SD}\right)$. For comparison, values are displayed in fold change to normalize for baseline lipid content and differences in surface covered by HPMs. All duplicates (D1 and D2) are an average of four images, expect for Trained-1 D2 (two images).

These data show that it takes approximately 10 hours to reach maximal lipid accumulation in HPMs from this trained individual after one addition of FAs. When designing a double labelled experiment, the incubation time can therefore be carefully planned as it may take 10 hours to form the maximal number of preformed LDs after the first incubation. It depends on the research question when the second incubation is scheduled (i.e. during lipid accumulation or during the plateau). For the next experiments we choose 12 hours for both incubations. 

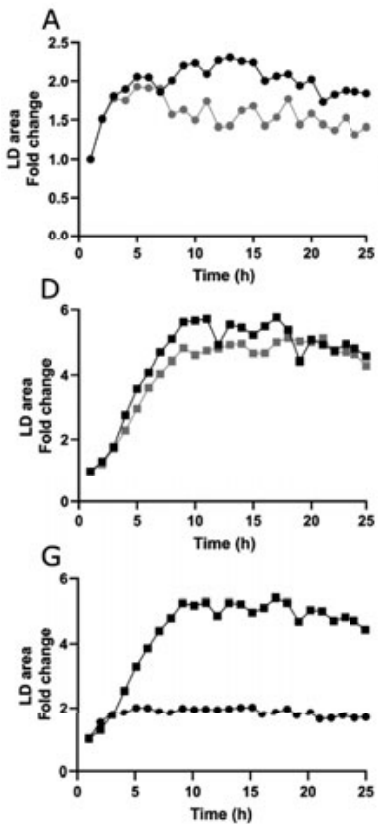
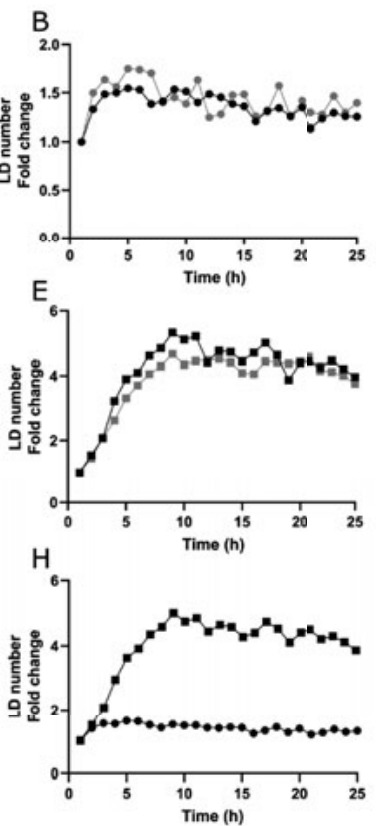

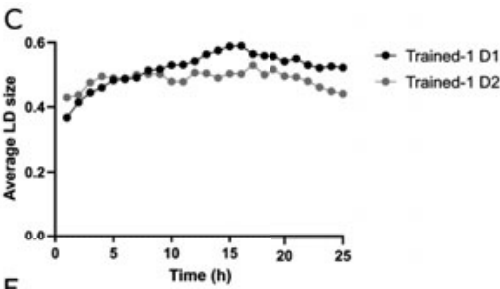

F
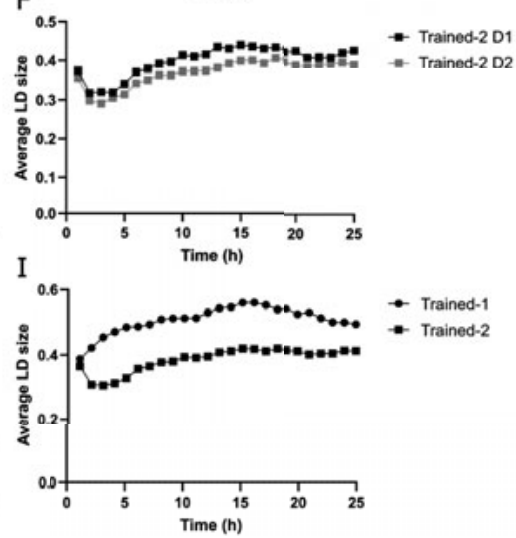

Figure 6. Time course analysis of lipid incorporation into primary myotubes of trained individuals. Human primary myotubes were differentiated for 6 days and incubated with oleate $(50 \mu \mathrm{M})$ and Bodipy 493/503 at $T=0$. Images were captured every hour with a spinning disk confocal microscope. A-C) Duplicates (D1 and D2) of experiment 1 (Trained-1). Duplicates represents one well which is an average of four images (D1) or two images (D2) on different locations within a well. Graphs display fold change increase in lipid area (total Bodipy signal in an image) relative to time point 1 (A), fold change increase in lipid droplet $(L D)$ number (total $L D$ count in an image) relative to time point $1(B)$ and average $L D$ size $\left(\mu m^{2}\right)$ (C). D-F) Duplicates of experiment 2 (Trained-2), with fold change increase in $L D$ area (D), fold change increase in $L D$ number $(E)$ and average $L D$ size $(F)$. Duplicates represents one well which is an average of four images on different locations within a well. G-I) Fold change increase of $L D$ area (G), LD number $(H)$ and average $L D$ size (I) in myotubes from trained individuals over time (hours). Data points represent the average values of duplicate measurements (Trained 1: A-C, $n=6$ images; Trained-2: $D-F, n=8$ images). 


\section{Chapter 7}

\section{Incorporation of two fluorescent fatty acids results in a variety of lipid droplets}

Incubation of both fluorescent FAs can result in a variety of green, yellow and red LDs, indicating that there are preformed, incorporating and new LDs respectively (Figure 7A). Preformed LDs are formed during the first incubation and contain mainly the first fluorescent FA (green LDs in Figure 7A). These are called preformed as they represent the first LD reservoir and are formed before the second incubation with FAs. During the second incubation the preformed LDs can incorporate the new FAs (Bodipy 558/568-C12 in Figure 7A) and contain both fluorescent FAs with different concentrations. These LDs are defined as incorporating LDs (visualized as yellow LDs). LDs that do not (or barely) incorporate new FAs remain classified as preformed LDs (green LDs in Figure 7A). During the second incubation new LDs can be formed and contain mostly the second label (Bodipy 558/568-C12 in Figure 7A) and these are classified as new LDs and indicated as red LDs in Figure 7A. Initial results show that both fluorescent FAs are taken up by the HPMs and the order of incubation (first Bodipy FL-C12 or Bodipy 558/568-C12) does not seem to matter (Figure 7B and 7C). The end result is a spectrum of different LDs. Interestingly, cells display a heterogeneous uptake of the fluorescent FAs within one donor. 


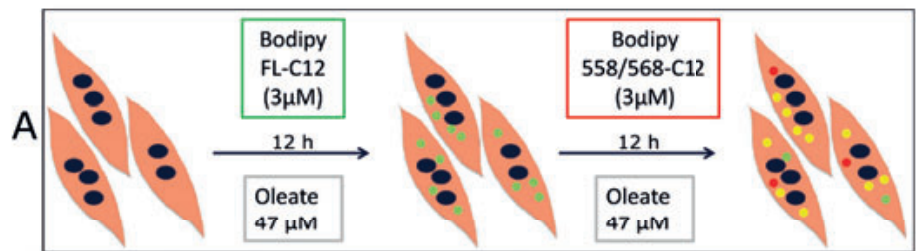

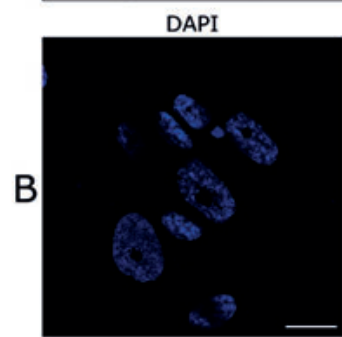

DAPI

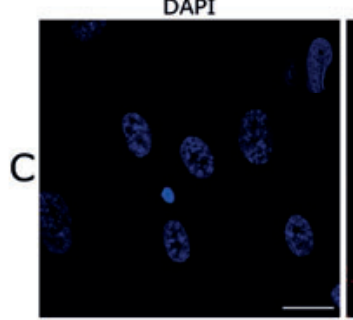

Bodipy FL-C12

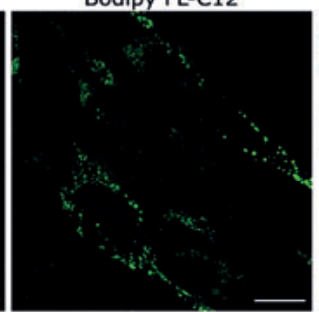

Bodipy 558/568-C12

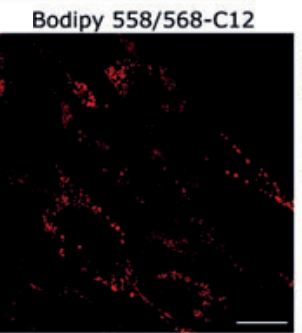

Bodipy FL-C12

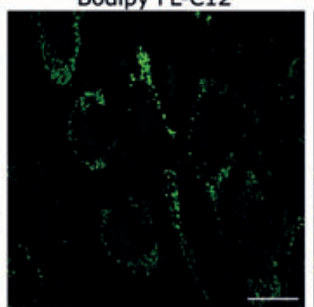

Merged

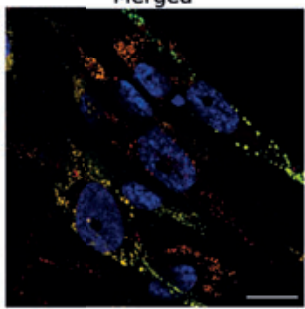

Merged

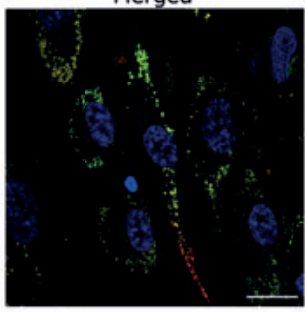

Figure 7. Fluorescent fatty acids are heterogeneously incorporated into human primary myotubes. A) Overall set-up of the experiment. Human primary myotubes are incubated first with $3 \mu M$ Bodipy FLC12 fatty acids (FAs) (green) for 12 hours along with $47 \mu \mathrm{M}$ oleate followed by a 12-hour incubation with $3 \mu \mathrm{M}$ Bodipy 558/568-C12 FAs (red) along with $47 \mu \mathrm{M}$ oleate. Incubation with both fluorescent FAs may result in a variety or red, green and yellow (both Bodipy labelled FAs present) lipid droplets (LDs). B-C) Differentiated human primary muscle cells from an untrained individual after a 12-hour incubation with 3 $\mu M$ Bodipy FL-C12 followed by a 12-hour incubation with $3 \mu$ M Bodipy 558/568-C12 (B) or the other way around (C). Incubation with both FAs result in different colours of LDs and a heterogeneously uptake of $L D$ s by the cells. $L D$ s can be classified as preformed $L D s$ (formed during the first incubation), incorporating $L D s$ (FAs from the second incubation incorporated into the preformed $L D s$ ) and the new $L D$ s (containing predominantly FAs from the second incubation). Nuclei are stained with DAPI (blue). Cells are fixed with $3.7 \%$ formaldehyde and imaged with a laser scanning confocal microscope. Scale bar $=20 \mu \mathrm{m}$. 


\section{Chapter 7}

\section{Quantification methods can distinguish between different lipid droplets}

With the current tool, we have shown that both Bodipy FL-C12 and Bodipy 558/568-C12 are present in all LDs formed, creating different kind of groups of LDs (preformed, incorporating and new LDs). Furthermore, we are able to quantify and visualize these groups as depicted in Figure 8. In this example, when the normalized mean intensity of Bodipy FL-C12 (green channel, Figure 8A) was plotted with the normalized mean intensity of Bodipy 558/568-C12 (red channel, Figure 8A) per LD, three clusters of LDs became apparent (Figure $\mathbf{8 B}$ ). Within this analysis we can distinguish between preformed, incorporating and new LDs and visualize them in the segmentation image (Figure 8D). Moreover, we are able to visualize the mean intensity ratio between the new and preformed LDs, whereas a high ratio (visualized as yellow/white in Figure 8D) means a higher fraction of newly provided FAs. In this example, 3 cells display different ratios, indicating that one cell contains relatively more new LDs, while another cell contains more preformed LDs. For each LD, the area (size) can be determined and the distribution of size can be visualized as well (Figure 8D, third image). A LD with a low Bodipy 558/568-C12/ Bodipy FL-C12 ratio (more preformed) is not necessarily larger than a LD with a higher ratio (Figure $8 \mathrm{C}$ ). 
A

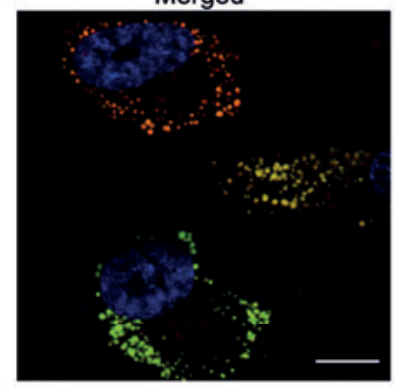

B

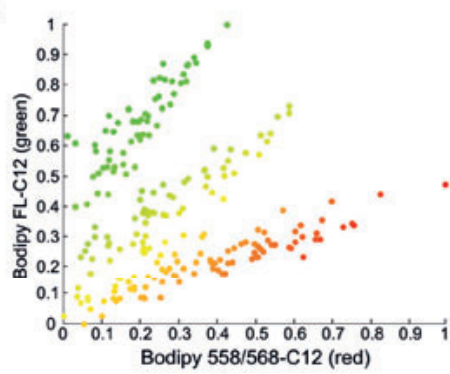

Bodipy FL-C12

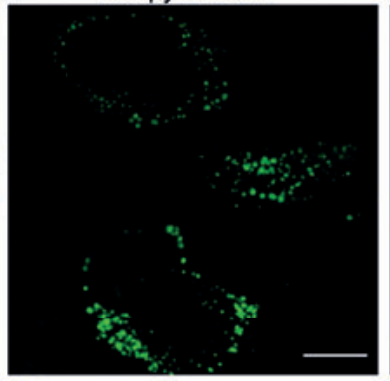

C
Bodipy $558 / 568-C 12$

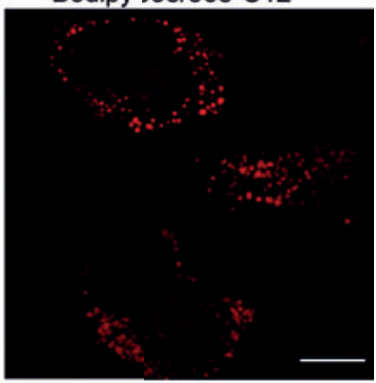

D
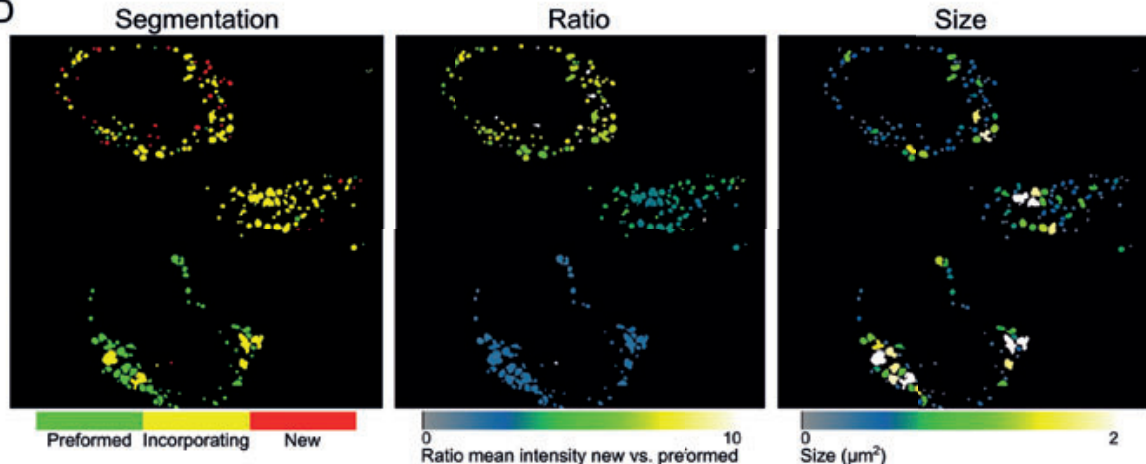

Figure 8. Quantification and visualization methods can distinguish between different clusters of lipid droplets. A) Example image of three different groups of lipid droplets (LDs): preformed LDs (predominantly containing Bodipy FL-C12), incorporating LDs (containing both Bodipy FL-C12 and Bodipy 558/568-C12) and new LDs (containing predominantly Bodipy 558/568-C12). Differentiated primary myoblasts from an untrained individual after a 12-hour incubation with $3 \mu \mathrm{M}$ Bodipy FL-C12 followed by a 12-hour incubation with $3 \mu M$ Bodipy 558/568-C12. Nuclei are stained with DAPI (blue). Cells are fixed with $3.7 \%$ formaldehyde and imaged with a laser scanning confocal microscope. Scale bar $=10 \mu \mathrm{m}$. B) Scatter plot of the normalized (min-max normalization) mean intensity of Bodipy FL-C12 (green) and Bodipy 558/568-C12 (red) of all LDs. Each dot represents a LD. Three clusters of LDs became apparent. C) Scatter plot of the ratio mean intensity Bodipy 558/568-C12/mean intensity Bodipy FL-C12 vs LD size $\left(\mu m^{2}\right)$. Each dot represents a LD. D) Visualization methods from binary images. Three groups can be formed within the analysis (preformed, incorporating and new $L D s$ ) and visualized in a segmentation overview. Next, a ratio can be displayed of the mean intensities of new/preformed LDs. A higher ratio illustrates a relative higher Bodipy 558/568-C12 content. This also reveals three clusters of $L D s$ and can be an important output to determine the fate of the fatty acids after incubation. Finally, the distribution of $L D$ size can be visualized. 


\section{Chapter 7}

\section{Differential lipid accumulation observed between patients with type 2 diabetes and trained individuals}

This microscopy tool has been developed to study LD dynamics in HPMs from insulin-sensitive individuals (e.g. trained athletes) and insulin-resistant individuals (e.g. patients with T2DM). To determine the timeframes for the first and second lipid incubation, lipid accumulation over time needs to be measured in HPMs cultured from donors with both these distinct phenotypes. Therefore, HPMs from a trained individual and a patient with T2DM were incubated with oleate and Bodipy 493/503 and followed over time. Interestingly, the myotubes from the trained individual accumulate FAs more rapidly, resulting in a higher lipid content compared to the HPMs from the T2DM patient (Figure 9A). The accumulation of lipids in both groups predominantly originates from an increase in LD number (Figure 9B), rather than an increase in LD size (Figure 9C). These observations were made in technical duplicates in HPMs from both donors (Figure 9D-F). In trained HPMs, lipid accumulation increases rapidly and there is a visual increase from $5 \mathrm{~h}$ to $21 \mathrm{~h}$ (Figure $9 \mathrm{~A}+\mathrm{G}$ ), while in HPMs from the patient, lipid accumulation did not differ much between these time points (Figure $\mathbf{9 A}+\mathbf{H}$ ). There was an absolute increase in lipid accumulation in the myotubes from the patient with T2DM (total LD area: $400.5 \mu \mathrm{m}^{2}(\mathrm{t}=1)$ vs 697.3 $\left.\mu \mathrm{m}^{2}(\mathrm{t}=10)\right)$, but this was much less compared to the myotubes from the trained individual (total LD area: $169.4 \mu \mathrm{m}^{2}(\mathrm{t}=1)$ vs $877.7 \mu \mathrm{m}^{2}(\mathrm{t}=10)$ ). These initial results suggest differential lipid accumulation in HPMs cultured from a trained individual versus an individual with T2DM. 

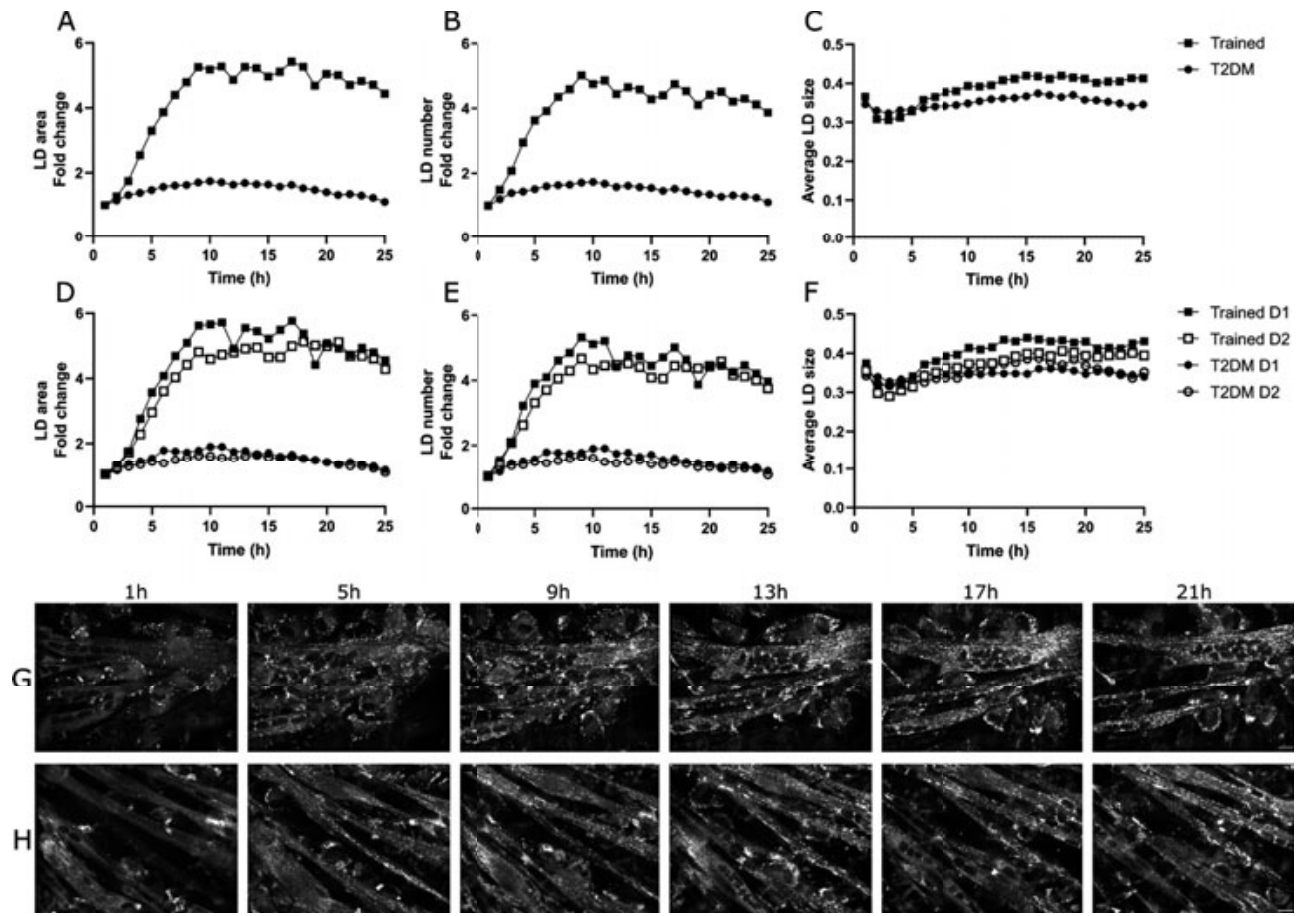

Figure 9. Lipid accumulation over time in myotubes from a trained individual and a patient with type 2 diabetes. Primary human myotubes were differentiated for 6 days and incubated with oleate $(50 \mu \mathrm{M})$ and Bodipy 493/503 at $t=0$. Images were captured every hour with a spinning disk confocal microscope. $A-C)$ Fold change increase in lipid droplet (LD) area (total Bodipy signal in an image) relative to time point $1(A)$, fold change increase in $L D$ number (total $L D$ count in an image) relative to time point 1 (B) and average $L D$ size $\left(\mu m^{2}\right)(C)$ in myotubes from trained or type 2 diabetes (T2DM) patient over time (hours). Data points represent the average values of duplicate measurements $(D-F)$ from one experiment $(n=8$ images). D-F) Technical duplicates (D1 and D2) represents 1 well which is an average of 4 images on different locations within a well. Fold change increase of $L D$ area $(D), L D$ number $(E)$ and average $L D$ size (F). G-H) Representing images of lipid accumulation in myotubes from a trained individual (G) and a T2DM patient $(H)$. Scale bar $=20 \mu \mathrm{m}$. 


\section{Chapter 7}

\section{Discussion}

In search for a tool to examine myocellular LD dynamics we have developed an in vitro model based upon cultured HPMs. In this model, we worked with confocal and livecell fluorescent microscopy to image LDs and the incorporation of two fluorescently labelled FAs therein. This chapter describes how the model was developed, how the proof-of-concept experiments were performed and the pioneering studies in HPMs grown from two phenotypically different donors (a trained athlete and a patient with T2DM). When developing this tool, we have checked for crosstalk, bleedthrough, FRET and colocalization with the neutral lipid stain MDH. Furthermore, time course experiments gave information for incubation periods and we proposed an optimal concentration of $3 \mu \mathrm{M}$ for Bodipy FL-C12 and Bodipy 558/568-C12 in HPMs. Custom-written ImageJ scripts were developed to quantify LD number, LD size and to distinguish between different pools of LDs (preformed, incorporating and new LDs) which are heterogeneously formed by HPMs.

Throughout the experiments in this chapter, we focused on keeping the total lipid load to the HPMs the same (i.e. the sum of the Bodipy labelled FA plus the vehicle FA oleate was always $50 \mu \mathrm{M}$ ). Depending on the type of experiment (examining crosstalk, bleed-through, FRET or colocalization with MDH staining), the concentration of Bodipy FL-C12 and Bodipy 558/568-C12 (and hence of oleate) varied between 2 and 16 $\mu \mathrm{M}$. For quantitative analysis, it obviously is important to use the same concentrations of Bodipy FL-C12 and Bodipy 558/568-C12 throughout the actual experiments. Our proof of principle experiments has led us to conclude that incubation with $3 \mu \mathrm{M}$ of the Bodipy labelled FAs gives reliable and reproducible results in HPMs.

To determine the optimal duration of incubation with the labelled FA, we assessed the time-course of incorporation of oleate by live-cell imaging. It was shown that it took approximately 10 hours to achieve a plateau in LD area in HPMs. It should be noted though that total lipid area is a reflection of net synthesis and degradation of TAGs in the LD and that we hence cannot conclude on the exact incorporation rate. Blockers of TAG synthesis (e.g. DGAT1 inhibitors) or TAG degradation (e.g. ATGL 
inhibitors) would be needed to determine FA incorporation rate more cleanly. During this plateau HPMs are still able to form new LDs, as we have shown in Figure 7 and 8. An initial incubation of HPMs with a Bodipy labelled FA of 12 hours, followed by incubation for another 12 hours with a FA with another label, not only resulted in the generation of new LDs (as evidenced by LDs predominantly contained the second fluorescent FA), but also into incorporation of the second FA into LDs that already were present (incorporating LDs). In these pioneering experiments, it appears that number of new LDs was lower than the number of incorporating LDs. Shortening the first incubation time ( $<10$ hours) will possibly result in a higher number of new LDs compared to longer incubation periods ( $>10$ hours), since lipid accumulation is high during the first few hours. For studying lipid incorporation into new or preformed LDs, an incubation time $<10 \mathrm{~h}$ is more feasible, as net lipid accumulation still takes place. Studying LD turnover can be achieved during longer incubation periods (>10 hours), since a plateau is formed and no net lipid accumulation takes place. In the ideal experiment when exploring putative differences in LD dynamics between different (groups of) donors, donor specific incorporation time courses are recommended to define the optimal duration of the incubation with the first labelled FA.

In our model with both fluorescent FAs, little cross-talk (the excitation of one fluorophore does also result in excitation of the other fluorophore resulting in dual emission) and to a greater extend bleed-through (the fluorescent emission of one fluorophore is also picked up within the emission window of the other fluorophore) was present in our experimental set-up. The putative bias originating from this could, however, be circumvented by sequential imaging of the labelled FAs. Thus, our experimental model is a valid tool to make the distinction between LDs that specifically incorporate one dye over the other, as well as to specifically identify LDs that incorporate both dyes. If two different fluorophores are present in close proximity (i.e. they are incorporated within the same LD), and if they do have overlapping spectra, false information may originate from FRET. Hence it is important to test if FRET takes place in the present study with both Bodipy FL-C12 and Bodipy 


\section{Chapter 7}

558/568-C12 used. Here we did not detect any significant FRET, indicating that the combination of the fluorophores used and their close proximity within the same LD does not affect the ability to validly identify the fluorophores and quantify the number and size of these LDs.

To ensure that the Bodipy labelled FAs were incorporated in all LDs, we combined imaging of the two labelled FAs with a neutral lipid dye which presumably stains all LDs (MDH) and computed colocalization using the Manders Colocalization Coefficients M1 and M2. As M1 and M2 computations are sensitive to background noise (Dunn et al., 2011), we performed M1 and M2 computations only after background correction by deconvolution. Colocalization measurements clearly revealed that all MDH positive LDs also contained a Bodipy labelled FA (M1=0.99) and vice versa (all Bodipy label containing LDs stained positive with MDH (M2=0.99)). This indicates a perfect colocalization and changes on the formation of LDs without fluorophores (i.e. only non-labelled oleate) is negligible.

Two fluorescent FAs were used throughout this chapter: Bodipy FL-C12 and Bodipy 558/568-C12 and have been used in other studies (Keeney et al., 2008; Wang et al., 2010; Rambold et al., 2015; Quinlivan et al., 2017). Both Bodipy FL-C12 and Bodipy 558/568-C12 have a carbon chain length of 12 carbon atoms and would initially be classified as median-chain FAs (Schönfeld \& Wojtczak, 2016). However, the Bodipy tag makes the total length of the FA more equivalent to a long-chain FA (Rambold et al., 2015; Kolahi et al., 2016; Kolahi et al., 2018). Irrespective of their fluorescent tag, both Bodipy FL-C12 and Bodipy 558/568-C12 are processed similarly and label the same subcellular structures (Quinlivan et al., 2017), as we have also observed in our experiments. However, Bodipy FL-C12 and Bodipy 558/568-C12 seems to be metabolized differently (Quinlivan et al., 2017), as Bodipy FL-C12 was more metabolized into polar lipids in this study, while Bodipy 558/568-C12 was more likely to be metabolized into TAGs. This might explain why the average M2 value (Bodipy label containing LD stained positive with MDH) of Bodipy 558/568-C12 was higher than Bodipy FL-C12 (0.95 vs 0.86). Although both fluorescent FAs showed high co-occurrence with neutral lipids and we performed two parallel experiments 
with either starting with Bodipy FL-C12 or with Bodipy 558/568-C12. Both orders are possible, although it is evidently important to remain constant throughout the experiment. Others have shown that Bodipy 558/568-C12 can be transported into the mitochondria and can be used as a substrate (Rambold et al., 2015). To our knowledge, it is not clear whether these fluorescent FAs are oxidized in the mitochondria. As the Bodipy-tag is attached to the methyl tail, it can be speculated that $\beta$-oxidation cannot fully operate.

With our newly developed tool, we can distinguish between preformed, incorporating and new LDs. Preformed LDs are developed during the first incubation, while new LDs are formed after the second incubation. Incorporating LDs are LDs that were present already after the first incubation, as evidenced by showing fluorescence of the first Bodipy labelled FA incubation, and still take up FAs from the second incubation. Interestingly, the uptake of FAs and therefore the distribution of preformed, incorporating and new LDs varied between HPMs. Some HPMs contained mainly preformed LDs, while other neighbouring cells had a mixture of incorporating and new LDs. The heterogeneously uptake of lipids has been observed previously in liver cells (Herms et al., 2013). To deal with excess FAs, either by supplying more FAs (current study) or by starvation (Rambold et al., 2015; Nguyen et al., 2017) cells increase the number LDs. This is essential to protect the cell against lipotoxicity (Olzmann \& Carvalho, 2019). Whether this protective buffer system is addressed as incorporating lipids into preformed LDs or formation of new LDs remains elusive. The ratio new LDs/preformed can be an important output to study the fate of fatty acids and hence LD dynamics. If lipids are more incorporated into preformed LDs than in new LDs, this can affect the ratio significantly. It will be interesting to study if this ratio differs among populations with different phenotypes, such as athletes versus patients with T2DM.

A live-cell experiment in HPMs derived from one trained individual and one patient with T2DM revealed preliminary data hinting towards faster and more incorporation in HPMs from a trained individual. This is in line with the lower FA incorporation into TAGs observed in myotubes from individuals with T2DM reported previously 


\section{Chapter 7}

(Sparks et al., 2014). Moreover, HPMs from a trained donor accumulated lipids due to an increase in LD number, rather than an increase in size (i.e. the size of the LDs from the trained donor were comparable to the size of the LDs in HPMs from the patient, while the increase in number was very different between these donors). According to the athlete's paradox, both endurance trained athletes and T2DM patients have high intramyocellular lipids, although they are metabolically very different (Goodpaster $e t$ al., 2001). Athletes are more metabolic flexible and are able to 'deal' with fat substrates more efficiently compared to insulin-resistant individuals (Kelley \& Mandarino, 2000; Galgani et al., 2008). A rapid increase in LD number may indicate the metabolic flexibility of the trained individual. The outcomes of these pioneering experiments are encouraging and hint towards differences in LD dynamics between the donors. Further studies with a larger sample size using the microscopy tool established in this work are needed to study differential lipid accumulation in insulin-resistant and insulin-sensitive individuals over time. Our custom written ImageJ script permits quantification of count and size of preformed, incorporating and new LDs and hence gives a reflection of LD dynamics.

We have successfully developed a novel microscopy-based tool to examine LD dynamics in HPM cultured from individuals with completely different metabolic phenotypes. This tool allows identification and quantification of myocellular lipids storage in vitro, to determine LD number, LD size and make the distinction between preformed, incorporating and new LDs when upon successive incubation with two different fluorescent FAs. We anticipate that with this tool, we will be capable to examine LD dynamics over time in more mechanistic detail than has previously been done. This tool will open avenues to move from predominantly correlative observations on how LDs may relate to insulin sensitivity towards identification of more causally related pathways. 


\section{Supplementary data for chapter 7}
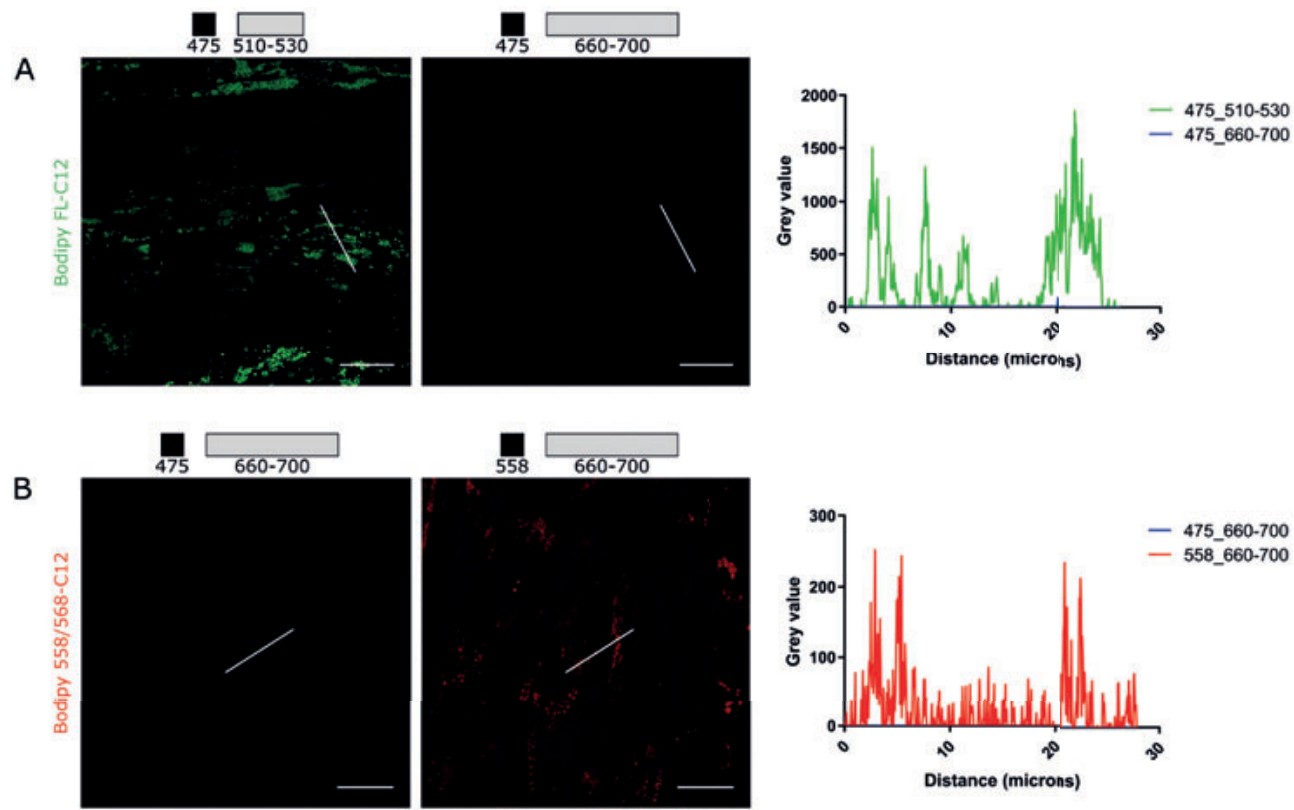

Figure S1. Fluorescence of Bodipy FL-C12 cannot be detected in the emission window of 660-700 and Bodipy 558/568-C12 cannot be excited with $475 \mathrm{~nm}$. A) Bodipy FL-C12 (6 $\mu \mathrm{M}+44 \mu \mathrm{M}$ oleate; 24hour incubation) is excited with $475 \mathrm{~nm}$ and fluorescence is detected between 510-530, but not between 660-700, also visualized in the graph. The graph represents the grey values of each picture, measured among the white line with a certain distance $(\mu \mathrm{m})$. B) Bodipy 558/568-C12 (2 $\mu \mathrm{M}+48 \mu \mathrm{M}$ oleate; 24-hour incubation) is excited with 475 and 558 with a emission window of 660-700 $\mathrm{nm}$. The graphs represent the grey values of each picture, measured among the white line with a certain distance $(\mu \mathrm{m})$. Scale bar $=20 \mu \mathrm{m}$. 


\section{References}

Badin PM, Langin D \& Moro C. (2013). Dynamics of skeletal muscle lipid pools. Trends Endocrinol Metab 24, 607-615.

Bergman BC, Perreault L, Strauss A, Bacon S, Kerege A, Harrison K, Brozinick JT, Hunerdosse DM, Playdon MC, Holmes W, Bui HH, Sanders P, Siddall P, Wei T, Thomas MK, Kuo MS \& Eckel RH. (2018). Intramuscular triglyceride synthesis: importance in muscle lipid partitioning in humans. American journal of physiology Endocrinology and metabolism 314, E152-E164.

Bergstrom J. (1975). Percutaneous needle biopsy of skeletal muscle in physiological and clinical research. Scandinavian journal of clinical and laboratory investigation 35, 609-616.

Brouwers B, Schrauwen-Hinderling Vera B, Jelenik T, Gemmink A, Havekes B, Bruls Y, Dahlmans D, Roden M, Hesselink Matthijs KC \& Schrauwen P. (2017). Metabolic disturbances of non-alcoholic fatty liver resemble the alterations typical for type 2 diabetes. Clinical Science 131, 1905-1917.

Daemen S, van Polanen N \& Hesselink MKC. (2018). The effect of diet and exercise on lipid droplet dynamics in human muscle tissue. J Exp Biol 221.

Dunn KW, Kamocka MM \& McDonald JH. (2011). A practical guide to evaluating colocalization in biological microscopy. Am J Physiol Cell Physiol 300, C723-C742.

Farese RV, Jr. \& Walther TC. (2009). Lipid droplets finally get a little R-E-S-P-E-C-T. Cell 139, 855-860.

Galgani JE, Moro C \& Ravussin E. (2008). Metabolic flexibility and insulin resistance. Am J Physiol Endocrinol Metab 295, E1009-1017.

Goodpaster BH, He J, Watkins S \& Kelley DE. (2001). Skeletal muscle lipid content and insulin resistance: evidence for a paradox in endurance-trained athletes. J Clin Endocrinol Metab 86, 5755-5761.

Herms A, Bosch M, Ariotti N, Reddy BJN, Fajardo A, Fernández-Vidal A, Alvarez-Guaita A, Fernández-Rojo MA, Rentero C, Tebar F, Enrich C, Geli M-I, Parton RG, Gross SP \& Pol A. (2013). Cell-to-cell heterogeneity in lipid droplets suggests a mechanism to reduce lipotoxicity. Curr Biol 23, 1489-1496.

Keeney DB, Lagrue C, Bryan-Walker K, Khan N, Leung TLF \& Poulin R. (2008). The use of fluorescent fatty acid analogs as labels in trematode experimental infections. Exp Parasitol 120, 15-20.

Kelley DE \& Mandarino LJ. (2000). Fuel selection in human skeletal muscle in insulin resistance: a reexamination. Diabetes 49, 677-683.

Kolahi K, Louey S, Varlamov O \& Thornburg K. (2016). Real-Time Tracking of BODIPY-C12 Long-Chain Fatty Acid in Human Term Placenta Reveals Unique Lipid Dynamics in Cytotrophoblast Cells. Plos One 11.

Kolahi KS, Valent AM \& Thornburg KL. (2018). Real-time microscopic assessment of fatty acid uptake kinetics in the human term placenta. Placenta 72-73, 1-9.

Nguyen TB, Louie SM, Daniele JR, Tran Q, Dillin A, Zoncu R, Nomura DK \& Olzmann JA. (2017). DGAT1-Dependent Lipid Droplet Biogenesis Protects Mitochondrial Function during Starvation-Induced Autophagy. Dev Cell 42, 9-21.e25.

Olzmann JA \& Carvalho P. (2019). Dynamics and functions of lipid droplets. Nat Rev Mol Cell Biol 20, 137-155.

Quinlivan VH, Wilson MH, Ruzicka J \& Farber SA. (2017). An HPLC-CAD/fluorescence 
lipidomics platform using fluorescent fatty acids as metabolic tracers. J Lipid Res 58, 1008-1020.

Rambold AS, Cohen S \& Lippincott-Schwartz J. (2015). Fatty Acid Trafficking in Starved Cells: Regulation by Lipid Droplet Lipolysis, Autophagy, and Mitochondrial Fusion Dynamics. Dev Cell 32, 678-692.

Schneider CA, Rasband WS \& Eliceiri KW. (2012). NIH Image to ImageJ: 25 years of image analysis. Nat Methods 9, 671-675.

Schönfeld P \& Wojtczak L. (2016). Short- and medium-chain fatty acids in energy metabolism: the cellular perspective. J Lipid Res 57, 943-954.

Sparks LM, Bosma M, Brouwers B, van de Weijer T, Bilet L, Schaart G, Moonen-Kornips E, Eichmann TO, Lass A, Hesselink MKC \& Schrauwen P. (2014). Reduced incorporation of fatty acids into triacylglycerol in myotubes from obese individuals with type 2 diabetes. Diabetes 63, 1583-1593.

Sparks LM, Moro C, Ukropcova B, Bajpeyi S, Civitarese AE, Hulver MW, Thoresen GH, Rustan AC \& Smith SR. (2011). Remodeling Lipid Metabolism and Improving Insulin Responsiveness in Human Primary Myotubes. Plos One 6, e21068.

van Loon LJC, Koopman R, Stegen JHCH, Wagenmakers AJM, Keizer HA \& Saris WHM. (2003). Intramyocellular lipids form an important substrate source during moderate intensity exercise in endurance-trained males in a fasted state. J Physiol 553, 611-625.

Vosselman MJ, Hoeks J, Brans B, Pallubinsky H, Nascimento EBM, van der Lans AAJJ, Broeders EPM, Mottaghy FM, Schrauwen P \& van Marken Lichtenbelt WD. (2015). Low brown adipose tissue activity in endurance-trained compared with lean sedentary men. International Journal of Obesity 39, 1696-1702.

Walther TC, Chung J \& Farese RV, Jr. (2017). Lipid Droplet Biogenesis. Annu Rev Cell Dev Biol 33, 491-510.

Wang HJ, Wei EH, Quiroga AD, Sun XJ, Touret N \& Lehner R. (2010). Altered Lipid Droplet Dynamics in Hepatocytes Lacking Triacylglycerol Hydrolase Expression. Mol Biol Cell 21, 1991-2000. 


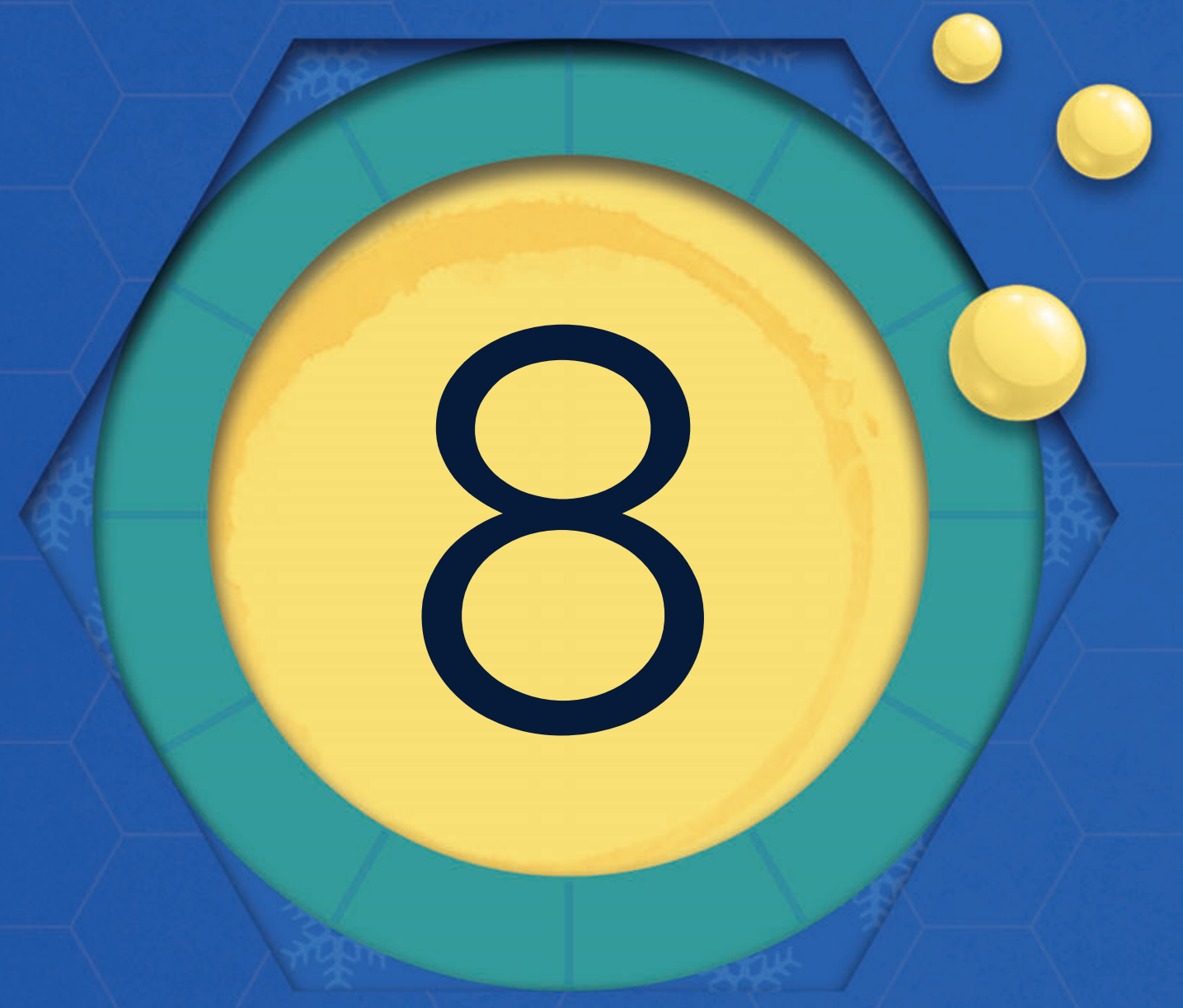

- 0 
General discussion 

Type 2 diabetes (T2DM) affects the quality of life of millions of people worldwide and understanding the hallmark of this disease, insulin resistance, is therefore crucial. Intramyocellular lipid (IMCL) storage is associated with insulin resistance in most populations, but this relation is not found in athletes who are insulin sensitivity despite also having high IMCL levels (a phenomenon called "athlete's paradox") (Goodpaster et al., 2001). IMCL is stored in the form of lipid droplets (LDs) which continuously, depending on energy demand, store lipids or provide fuel for energy production. This continuous turnover of lipids and the dynamic nature of LDs may be enhanced in athletes, possibly explaining the athlete's paradox. It is hypothesized that maintaining the dynamic nature of LDs is important to preserve muscle insulin sensitivity (Badin et al., 2013). The aim of this thesis was to examine the role of LD dynamics in relation to insulin sensitivity upon different human intervention studies. Therefore, we studied the effects on lipids and features of LD dynamics after cold exposure (chapter 3), circadian misalignment (chapter 4), resveratrol supplementation (chapter 5) and acute exercise (chapter 6).

\section{Which factors of lipid droplet dynamics can influence insulin sensitivity?}

IMCL content has been associated with insulin resistance in non-athletes. As described in the athlete paradox, total IMCL content does not cause insulin resistance per se and prompted us to look further into detailed features of IMCL. Parameters such as LD number, size, muscle fibre type, subcellular location, PLIN5 coating and

lipid composition are affected by exercise, nutrient availability or differ between individuals with various metabolic phenotypes (Gemmink et al., 2016; Shepherd et al., 2017; Daemen et al., 2018a). These parameters describe the dynamic nature of LDs and might better explain the relationship between IMCL and insulin resistance.

\section{Number and size of lipid droplets}

The number of LDs is positively correlated with insulin sensitivity (Daemen et al., 2018a) and insulin-resistant individuals display a lower number of LDs and their 


\section{Chapter 8}

LDs are also increased in size. Size has, in contrast to LD number, been negatively associated with insulin sensitivity (He et al., 2004; Nielsen et al., 2017). Studying both the number and size of LDs is important as individuals with similar levels of IMCL may display differences in LD number or size (numerous small LDs vs fewer but larger LDs). In chapter 5 and $\mathbf{6}$, we observed an increase in LD number after resveratrol supplementation and 4 hours post-exercise respectively, while size was not profoundly affected. This is in line with a proposed 'athlete-like phenotype' (chapter 5 and (Gemmink et al., 2020)), characterized by high levels of IMCL without impeding insulin sensitivity. This athlete-like storage profile is characterized by numerous small LDs particularly in type I muscle fibres. In general, endurance trained athletes have more type I fibres compared with untrained individuals (Wilson et al., 2012) and type I muscle fibres have a higher number of LDs compared with type II muscle fibres (Nielsen et al., 2017; Daemen et al., 2018a). Thus in trained athletes, a higher fraction of total IMCL is dispersed in small LDs in type I muscle fibres, a storage pattern that is not associated with impeded insulin sensitivity and hence will be referred to as benign lipid storage.

\section{Muscle fibre type}

Many studies have recorded differences in myocellular lipid content between muscle fibre types (He et al., 2001; Malenfant et al., 2001; van Loon et al., 2003; Shaw et al., 2008; Shepherd et al., 2017; Daemen et al., 2018b), where type I muscle fibres display a higher IMCL content compared with type II muscle fibres. This also observed in the present thesis (chapter 4, 5 and 6). This matches the phenotypical characteristics of type I muscle fibres being more oxidative, while type II muscle fibres are more glycolytic. Type I muscle fibres have a high capillary supply and mitochondrial content and rely mainly on fatty acids (FAs) as substrates. This may explain why type I muscle fibres store more LDs. Endurance-trained athletes have both high levels of IMCL and a higher percentage type I muscle fibres (Wilson et al., 2012). Aerobic exercise training of low or moderate intensity increases the rely on lipid metabolism (Sidossis et al., 1998) probably promoting IMCL storage and LD turnover within the 
muscle fibres. Hence, a detailed fibre type distinction in IMCL analysis is warranted. In fact, the effects we observed in LD characteristics after resveratrol supplementation (chapter 5) and acute exercise (chapter 6) were specific for type I muscle fibres.

\section{Subcellular location}

In the present thesis we have also examined the subcellular location of LDs within the muscle. LDs that are close to the sarcolemma are indicated as subsarcolemmal (SS) LDs. LDs that are located between the myofibrils (contractile elements) are indicated as intermyofibrillar (IMF) LDs. Location of these different LDs suggest a different role of the LDs. LDs close to the sarcolemma are in close proximity with the vascular system and are therefore more likely to be influenced by high plasma lipid levels. LDs between myofibrils can function as an energy source to the IMF mitochondria, which are important to generate ATP during muscle contraction (Hood, 2001). As insulin signalling starts at the insulin receptor in the SS area, bioactive SS lipids are hypothesized to interfere with insulin signalling and therefore impede insulin sensitivity. Indeed, SS bioactive lipids were especially associated with insulin resistance (Perreault et al., 2018). Therefore, SS LDs are theoretically more related to impaired insulin sensitivity than IMF LDs.

Trained individuals have a higher IMCL content in the IMF region compared with sedentary individuals (Daemen et al., 2018a), indicating a role of IMF LDs in exercise. During acute exercise, the IMF LDs are especially used in trained athletes (Koh et al., 2017; Jevons et al., 2020). For non-athletes the results are not that consistent. Long term exercise programs decreased IMCL in SS LDs (Nielsen et al., 2010; Devries et al., 2013; Samjoo et al., 2013; Li et al., 2014) with no change in IMF LDs (Nielsen et al., 2010; Devries et al., 2013; Li et al., 2014), or an increase in IMF LDs (Samjoo et al., 2013). This may be due to the fact that sedentary populations, especially patients with T2DM have much more SS LDs (Nielsen et al., 2010; Daemen et al., 2018a) and this number will be reduced by training. Mitochondrial content increased in both regions (Devries et al., 2013; Samjoo et al., 2013) or more in the SS region (Nielsen et al., 2010), indicating a metabolic role for both regions during exercise. For acute exercise, in chapter 6 we 


\section{Chapter 8}

described a decrease in SS LDs in young lean inactive individuals, which was not observed by others (Chee et al., 2016), who rather reported a decrease in IMF LDs upon exercise in young lean active individuals. Perhaps the differences in activity level (and thereby $\mathrm{VO}_{2} \mathrm{max}$ ) can explain the contrasting outcomes between these young individuals. Four hours post-exercise, we observed a replenishment in the SS LDs, probably fuelled by the relatively high plasma NEFA levels combined with a lower fat oxidation. It should be noted that the exchange of lipids between IMF and SS LDs cannot be excluded. In chapter 6, before exercise IMF LDs made up of $83 \%$ of the total IMCL pool, and SS LDs the remaining 17\%. Just after exercise, this percentage was increased in the IMF region (and decreased in the SS region) and restored back to resting values during recovery. A shift in relative distribution was also observed in elite triathletes, but in this case a decrease in IMF directly post-exercise (Jevons et al., 2020). This shows that the relative distribution of lipid content within these pools are dynamic and can change after exercise and during recovery. More research is needed to study the contribution of IMF and SS LDs during exercise in inactive individuals, especially during and after an acute exercise bout, as well as possible exchange of lipids between these pools, which might explain the unexpected drop in SS LDs directly after exercise in inactive young individuals observed in chapter 6.

\section{PLIN5 coating}

LDs consist of a phospholipid monolayer which is coated with different kinds of proteins, such as the proteins of the perilipin (PLIN) family. PLIN1 is primarily found in adipose tissue, while PLIN2 (Shaw et al., 2012; Shepherd et al., 2012; Daemen et al., 2018a; Gemmink et al., 2018b) and PLIN3 (Louche et al., 2013; Covington et al., 2015; Shepherd et al., 2017) can be found in the human muscle tissue. Little is known about PLIN4, but in some studies it was also observed in human muscle tissue (Pourteymour et al., 2015; Shepherd et al., 2017). The most studied LD coating protein in muscle is PLIN5, where PLIN5 seems to be involved in both lipid storage and lipid oxidation (Bosma et al., 2013; Shepherd et al., 2017; Gemmink et al., 2018b). 
In chapter 5 and 6, we observed an increase in PLIN5 positive LDs (PLIN5+ LDs) after resveratrol supplementation, and four hours post-exercise respectively. The increase of PLIN5+ LDs is probably due to a redistribution of the cytosolic PLIN5 pool towards the LD bound pool (Wolins et al., 2006; Gemmink et al., 2016; Shepherd et al., 2017; Jevons et al., 2020). This is also in line with chapter $\mathbf{5}$, where we observed no changes in total PLIN5 content, but an increase in PLIN5+ LDs, indicating a redistribution of PLIN5. PLIN5 is a frequently studied protein and interesting in terms of oxidative metabolism, because of its potential dual role in lipid storage and lipid oxidation and its putative role in LD-mitochondrial interaction (Bosma et al., 2012; Benador et al., 2018; Gemmink et al., 2018b). Both PLIN5 protein content and PLIN5+ LDs are higher in insulin-sensitive athletes compared with insulin-resistant individuals (Gemmink et al., 2018a). Furthermore, a preferred utilization of PLIN5+ LDs during exercise was observed (Shepherd et al., 2013) as well as storage in PLIN5+ LDs alleviated fastinginduced insulin resistance (Gemmink et al., 2016). The data presented in this thesis and by others therefore suggest an important role for PLIN5 in LD dynamics. The direct role of PLIN5 in LD dynamics and its effects on insulin sensitivity in humans remains elusive.

\section{Mitochondrial function}

Mitochondria are essential for lipid metabolism. Mitochondrial function can be studied by high-resolution respirometry in muscle fibres from muscle biopsies. This is a powerful method to study mitochondrial function ex vivo and we have used this in chapter 3. In chapter 3, we did not observe changes in mitochondrial function upon acute cold exposure (without shivering). Perhaps longer duration or the induction of shivering would result in increased mitochondrial respiration. It has been recently proposed that mild cold-shivering is required to stimulate muscle activation, and therefore muscle mitochondrial function and muscle insulin sensitivity (Remie et al., 2021). As mitochondria use LD-derived FAs for oxidation, mitochondria and LD dynamics are functionally associated determinants of insulin sensitivity. Insulinresistant individuals are characterised by lower mitochondrial function and a 


\section{Chapter 8}

disrupted mitochondrial network, associated with lower mitochondrial oxidative capacity (Houzelle et al., 2021). Exercise increases mitochondrial function and the interaction between LDs and mitochondria (Tarnopolsky et al., 2007; Koh et al., 2017). If LD-mitochondrial interactions differ between insulin-sensitive and insulin-resistant individuals and whether it can be improved by a stimulus (e.g., cold exposure) needs to be investigated. Moreover, the exact role of PLIN5 in the LD-mitochondria interaction is not yet known.

\section{Lipid composition}

LDs mainly consist of triacylglycerols (TAGs) and cholesterol esters. Lipids stored within a LD are suggested to be non-toxic for the cell, whereas bioactive lipids in the cytosol are associated with cell dysfunction (Listenberger et al., 2003). Thus, to study the role of LDs in disturbed lipid accumulation, research has been focused on lipid species and the relation with insulin resistance. The most studied bioactive lipids are diacylglycerols (DAGs) and ceramides. As we discussed in chapter 2, the role of DAGs and ceramides are still contradictive, as some studies find a relation with insulin resistance (Straczkowski et al., 2004; Moro et al., 2009; Szendroedi et al., 2014), while other studies fail to do so (Skovbro et al., 2008; Vistisen et al., 2008; Anastasiou et al., 2009). In chapter 4, we performed a semi-targeted lipidomics approach to examine which lipid species were affected by circadian misalignment and the associated reduction in insulin sensitivity. In this chapter we did not observe changes in any of the DAGs or ceramides. Thus, it is not clear from this misalignmentinduced reduction in insulin sensitivity if these lipid species interfere with insulin sensitivity. Interestingly, cardiolipin, a mitochondrial inner membrane specific lipid, was reduced upon circadian misalignment. Reduced levels of cardiolipin are associated with lower mitochondrial content and function (Houtkooper \& Vaz, 2008), although we could not confirm that in our study (chapter 4). One explanation could be that the misalignment protocol was too short (3.5 days) to detect changes in mitochondrial function. Whether long term misalignment or shift work also results in reduced cardiolipin levels needs to be examined. 
Another observation was an increase in longer TAG species ( $\geq 55$ total carbon atoms) upon circadian misalignment, indicating oxidation of shorter chain FAs and/ or FA elongation (chapter 4). This pattern of longer TAG species was in line with the observed changes in gene sets of FA elongation upon circadian misalignment (Wefers et al., 2018). Moreover, some elongation enzymes show a circadian rhythm (Loizides-Mangold et al., 2017) and an increase in elongation enzymes was observed after disruption of clock genes (Hodge et al., 2015). This indicates that elongation of FAs is controlled by the circadian clock. The relation between very long FAs ( $>18$ carbon atoms) and insulin sensitivity is not yet fully understood. Based on our data from chapter 4 , it is tempting to suggest a positive association between very long-chain FAs and impaired insulin sensitivity. However, others found a positive association between chain length and a decreased risk of T2DM (Rhee et al., 2011), or did not observe changes in very long-chain FAs upon weight reduction (Schwab et al., 2008). The level of saturation of FAs is another factor that is associated with insulin resistance (Kahn et al., 2021), and could therefore potentially explain the differences between studies. Thus, more studies are needed to study the direct link of chain length, especially very long-chain FAs, with insulin sensitivity while taking the saturation into account.

Whether there are also changes in chain length within the LDs cannot be concluded from our results (chapter 4) as the lipidomics approach is based on the whole muscle lysate. With this method, one cannot determine which lipids are present in the LDs, cytosol or in other lipid structures, such as membranes. Other techniques like coherent anti-Stokes Raman scattering (CARS) microscopy have been used to look at lipid composition inside the LDs of muscle tissue from insulin-sensitive and resistant individuals (Daemen et al., 2020). In this study modest but significant effects on FA chain length were observed hinting towards longer FAs in large LDs and in subsarcolemmal LDs, typically the LDs that are associated with insulin resistance. Overall, the contribution of lipid composition to insulin resistance remains elusive. 


\section{Studying lipid droplet dynamics}

In chapter 4, 5, and 6, confocal fluorescent microscopy was used to measure characteristics of LD dynamics in human muscle tissues. With confocal microscopy, fluorophores are used to obtain static information on a dynamic process and detailed information of LDs e.g., LD size, number, distribution and coating with PLIN5. Changes in these features before and after an intervention provide an indication as to whether or not these LDs are responsive to interventions (and possess dynamic behaviour). In chapter 6 we applied this approach to obtain (indirect) indications of LD dynamics in human tissue before exercise, directly after exercise and four hours post-exercise. To determine LD dynamics more directly, lipid accumulation can also be studied in living cultured cells over time as shown in chapter 7 . Studies in cultured human muscle cells can provide more mechanistic insights in determining LD dynamics. Time course analysis of changes in LD features can provide a more dynamic examination of LD dynamics.

In chapter 7, we describe the pioneering steps in setting up a tool to study LD dynamics in human primary myotubes (HPMs). This tool makes it feasible to study the time course of lipid incorporation, and to discriminate between LD pools that are rather static from more dynamic LD pools in cultured HPMs from different donors. The experiments described in chapter 7 with two labelled FAs can facilitate to further explore the 'athlete-like storage' that was described in chapter 5. As mentioned before, athletes have a higher turnover of lipids and are associated with a higher number of smaller LDs in type I muscle fibres, while patients with T2DM have fewer larger LDs in the SS region in type II muscle fibres (Gemmink et al., 2020). Based on these observations, we expect (with this tool) that patients with T2DM mainly store the lipids into preformed (existing) LDs, resulting in large static LDs whereas athletes are able to make new LDs and effectively oxidize the substrate, which result in more and smaller LDs. This would result into a higher ratio (new/preformed) in the myotubes from athletes. Furthermore, we can determine which LDs are more dynamic by investigating the ratio of two different fluorescently labelled FAs within the LD. Subsequently, the tool could be further used to study the utilization 
of the LDs after a stimulus (e.g. activation of lipolysis or interventions that induce fat oxidation) and trace the fate of the fluorescent FAs in combination with other dyes. Others have observed the transfer of fluorescently labelled FAs from LDs into mitochondria in starved mouse embryonic fibroblasts (Rambold et al., 2015). If this is also the case in HPM needs to be examined. In this way we can further examine the relation between LD dynamics and the metabolic differences between insulinresistant individuals and insulin-sensitive individuals. With this tool, we expect to examine more mechanistic details of LD dynamics than has previously been studied. Furthermore, it can contribute towards understanding the more causally related pathways, rather than correlative observations between LD dynamics and insulin sensitivity.

\section{Other techniques}

In this thesis, we utilized fluorescent microscopy techniques to study LDs in human muscle tissue. Although highly valuable to localize and characterize LDs, there are some drawbacks, as the techniques are laborious and do not provide high-throughput. To study LD dynamics in humans, muscle biopsies are required which is invasive for participants and patients. MRI and MRS are non-invasive techniques to determine IMCL content in human participants (Li et al., 2008). However, it does not provide the detailed information about LDs, such as number, size, location and PLIN5 coating. These features are essential to examine characteristics of LD dynamics.

Besides fluorescent microscopy, electron microscopy (EM) is also a commonly used imaging technique to determine LD characteristics and LD localisation (SS or IMF region). The high resolution of EM can be used to visualize the interaction of LDs with mitochondria (Tarnopolsky et al., 2007; Nielsen et al., 2010). The specificity of fluorescent microscopy and the high resolution of EM can be combined in correlative light and electron microscopy (CLEM). This technique had been used to study the interaction of LDs with mitochondria in relation to PLIN5 localisation (Gemmink et al., 2018b). This technique is, however, time consuming and difficult in terms of sample preparation and image correlation (Begemann \& Galic, 2016). A challenge 


\section{Chapter 8}

for EM is the determination of muscle fibre types, which is in most cases more demanding (based on the thickness of the z-line and mitochondrial content (Koh et al., 2017)) and quite easily visualised with fluorescent microscopy. In addition, the sample preparation used for EM does not allow live-cell imaging.

\section{Limitation of the studies}

One limitation of the studies outlined in this thesis is that most studies were focused on male individuals. Men and women differ in terms of IMCL storage and lipid metabolism and the effects we observed in men, cannot directly be translated to women. Many studies have reported higher levels of IMCL in women compared with men (Tarnopolsky et al., 1990; Roepstorff et al., 2002; Steffensen et al., 2002; Perreault et al., 2010; Høeg et al., 2011). This could be explained by higher NEFA levels (Goossens et al., 2021), higher postprandial TAG clearance (Horton et al., 2002) or higher fraction of type I muscle fibres observed in women (Steffensen et al., 2002; Høeg et al., 2009). As mentioned previously, type I muscle fibres typically store higher amounts of lipids compared with type II muscle fibres. The exact reason of the sex differences in IMCL still needs to be resolved.

Despite higher IMCL levels, women are not per se less insulin-sensitive (Perreault et al., 2010). In fact, healthy lean women have a higher muscle insulin sensitivity compared with men when matched for age, physical fitness and BMI (Nuutila et al., 1995), and they also have a lower risk of developing T2DM (Sattar, 2013). Moreover, women are less sensitive to acute lipid-induced insulin resistance than men (Høeg et al., 2011). Interestingly, the higher IMCL content in women is due a higher number of LDs, rather than size (Devries et al., 2007; Tarnopolsky et al., 2007). Furthermore, the percentage of LD-mitochondria contact is increased in women after exercise, but not in men (Devries et al., 2007). It is also suggested that women have a higher PLIN5 content than men (Peters et al., 2012). These results suggest that women have an enhanced level of LD dynamics compared with men, which could explain the preserved insulin sensitivity. 
The lipid storage described in women is very comparable to the proposed athletes like storage of IMCL (chapter 5), with a 'benign' IMCL storage in numerous small LDs, in contact with mitochondria and coated with PLIN5, without impeding insulin sensitivity (Gemmink et al., 2020). It would therefore be interesting to compare the features of LD dynamics between men and women, to get more insight into the sex differences of IMCL storage and the relation to insulin sensitivity. Most studies that compare men and women are performed in young healthy individuals (both active and inactive) and if the discrepancy between sexes is also present in insulin-resistant individuals is not yet known. In the progression to T2DM diabetes, diminished insulin sensitivity was related to altered IMCL metabolism in men, but not in women (Perreault et al., 2010), suggesting that the changes of IMCL in the development of insulin resistance are more relevant for men than for women. Although interventions to improve lipid metabolism are still relevant for women (Schenk \& Horowitz, 2007) different effects between sexes should be a topic for future studies.

\section{Concluding remarks and future perspectives}

With this thesis we have shown that lipid storage is not an inert source of fuel upon demand, but highly dynamic. Lipid metabolism is complex and dependent on many factors such as internal storage, substrate availability and energy demand. LDs store and sequester lipids to prevent lipotoxicity within the cell and provide lipids on demand. Preservation of the dynamic nature of LDs is essential to maintain metabolic health and disturbances can affect insulin sensitivity. The main aim of this thesis was to study intramyocellular LD dynamics in relation to insulin sensitivity. When studying LD dynamics, some parameters are important to consider. LDs vary in number and size and these are both differently associated with insulin sensitivity. Therefore, it is important to look at the morphology of the LDs. Type I muscle fibres have higher IMCL content and these are stored subcellular on different locations, suggesting they may serve different functions. PLIN5 is proposed to be involved in lipid storage and delivering FAs to mitochondria for oxidation. Furthermore, specific lipid species are altered in compromised insulin sensitivity, suggesting 


\section{Chapter 8}

the contribution of lipid composition in the development of insulin resistance. Microscopy techniques have been used to study many features of LD dynamics, and live-cell fluorescent microscopy is a promising tool to get more mechanistic insight into LD dynamics over time. It is still not clear what specific aspect of LD dynamics can be targeted to improve insulin sensitivity and several questions remain unanswered. Future research can focus on the following aspects:

- LD storage profile observed in athletes is considered benign, as the high levels of IMCL are stored such that insulin sensitivity is not impaired. It is important to explore how human interventions, such as exercise and diet, can change the LD profile to a more benign profile to promote metabolic health.

- Women have higher levels of IMCL compared with men, but remain insulinsensitive (Perreault et al., 2010). This proposes a different lipid storage in women compared with men, possible comparable to an athlete-like storage. It would be interesting to compare the LD profiles of sedentary women and men in terms of LD size, number, subcellular location and PLIN5 coating and whether these differences are diminished when both sexes are trained or insulin-resistant.

- Resveratrol can be used to modulate LD storage profiles comparable to what have been observed in athletes, as we have shown in chapter 5 . Larger studies are needed to confirm this modulation and in comparison with an exercise intervention. In chapter 5, we observed that resveratrol could induce lipid accumulation in vitro and in vivo. Mechanistic studies are needed to explore if this is due to diminished lipid oxidation or other alterations in lipid metabolism. Resveratrol has been observed to improve mitochondrial function and increase oxidative capacity (Timmers et al., 2011; Timmers et al., 2016; de Ligt et al., 2018), but studies on lipid turnover are warranted to understand its role in lipid accumulation. The role of resveratrol on PLIN5 is yet unknown in humans. We observed an induction of PLIN5+ LDs upon resveratrol supplementation. Future research is needed to study the effects of resveratrol of PLIN5 content in relation to LD storage and lipid oxidation. 
- Subcellular location of LDs has shown to be important, as insulin-resistant individuals store IMCL mainly in SS LDs in type II muscle fibres and insulinsensitive individuals store IMCL mainly in IMF LDs in type I muscle fibres. We and others have shown that the relative distribution of IMCL in the IMF and SS region is different pre- versus post-exercise and that the post-exercise pattern is profoundly affected by differences in circulatory NEFAs. The dynamic interaction between IMF LDs and SS LDs needs to be resolved to better understand the intracellular lipid flux during exercise to get a greater understanding of lipid metabolism.

- Changes in lipid species can contribute to insulin sensitivity (Bergman \& Goodpaster, 2020). In chapter 4, we used a semi-targeted lipidomics approach to study changes in lipid species after circadian misalignment and observed a decrease in cardiolipins and an increase in longer TAG species. Future studies should examine how specific lipid classes, such as cardiolipins and very longchain FAs directly affect insulin sensitivity and contribute to the development of muscle insulin resistance.

- Our preliminary data has shown that insulin-sensitive HPMs accumulate lipids faster compared with HPM from an insulin-resistant donor. Our microscopy tool can be used to further explore region-specific incorporation of labelled FAs into different pools. Further experiments are needed to track FAs on different time points in fixed cells or follow in time in live-cell analysis and whether this differs between donors with distinct metabolic phenotypes. Future research needs to examine LD dynamics over time and in more detail to determine how LDs can causally affect insulin sensitivity. 


\section{References}

Anastasiou CA, Kavouras SA, Lentzas Y, Gova A, Sidossis LS \& Melidonis A. (2009). Diabetes mellitus is associated with increased intramyocellular triglyceride, but not diglyceride, content in obese humans. Metabolism 58, 1636-1642.

Badin PM, Langin D \& Moro C. (2013). Dynamics of skeletal muscle lipid pools. Trends Endocrinol Metab 24, 607-615.

Begemann I \& Galic M. (2016). Correlative Light Electron Microscopy: Connecting Synaptic Structure and Function. Front Synaptic Neurosci 8, 28-28.

Benador IY, Veliova M, Mahdaviani K, Petcherski A, Wikstrom JD, Assali EA, Acín-Pérez R, Shum M, Oliveira MF, Cinti S, Sztalryd C, Barshop WD, Wohlschlegel JA, Corkey BE, Liesa M \& Shirihai OS. (2018). Mitochondria Bound to Lipid Droplets Have Unique Bioenergetics, Composition, and Dynamics that Support Lipid Droplet Expansion. Cell Metabolism 27, 869-885.e866.

Bergman BC \& Goodpaster BH. (2020). Exercise and Muscle Lipid Content, Composition, and Localization: Influence on Muscle Insulin Sensitivity. Diabetes 69, 848-858.

Bosma M, Minnaard R, Sparks LM, Schaart G, Losen M, de Baets MH, Duimel H, Kersten S, Bickel PE, Schrauwen P \& Hesselink MKC. (2012). The lipid droplet coat protein perilipin 5 also localizes to muscle mitochondria. Histochemistry and Cell Biology 137, 205-216.

Bosma M, Sparks LM, Hooiveld GJ, Jorgensen JA, Houten SM, Schrauwen P, Kersten S \& Hesselink MK. (2013). Overexpression of PLIN5 in skeletal muscle promotes oxidative gene expression and intramyocellular lipid content without compromising insulin sensitivity. Biochim Biophys Acta 1831, 844-852.

Chee C, Shannon CE, Burns A, Selby AL, Wilkinson D, Smith K, Greenhaff PL \& Stephens FB. (2016). Relative Contribution of Intramyocellular Lipid to Whole-Body Fat Oxidation Is Reduced With Age but Subsarcolemmal Lipid Accumulation and Insulin Resistance Are Only Associated With Overweight Individuals. Diabetes 65, 840-850.

Covington JD, Noland RC, Hebert RC, Masinter BS, Smith SR, Rustan AC, Ravussin E \& Bajpeyi S. (2015). Perilipin 3 Differentially Regulates Skeletal Muscle Lipid Oxidation in Active, Sedentary, and Type 2 Diabetic Males. The Journal of Clinical Endocrinology \& Metabolism 100, 3683-3692.

Daemen S, Gemmink A, Brouwers B, Meex RCR, Huntjens PR, Schaart G, Moonen-Komips E, Jorgensen J, Hoeks J, Schrauwen P \& Hesselink MKC. (2018a). Distinct lipid droplet characteristics and distribution unmask the apparent contradiction of the athlete's paradox. Mol Metab 17, 71-81.

Daemen S, Gemmink A, Paul A, Billecke N, Rieger K, Parekh SH \& Hesselink MKC. (2020). Label-free CARS microscopy reveals similar triacylglycerol acyl chain length and saturation in myocellular lipid droplets of athletes and individuals with type 2 diabetes. Diabetologia 63, 2654-2664.

Daemen S, van Polanen N \& Hesselink MKC. (2018b). The effect of diet and exercise on lipid droplet dynamics in human muscle tissue. J Exp Biol 221.

de Ligt M, Bruls YMH, Hansen J, Habets MF, Havekes B, Nascimento EBM, Moonen-Kornips E, Schaart G, Schrauwen-Hinderling VB, van Marken Lichtenbelt W \& Schrauwen P. (2018). Resveratrol improves ex vivo mitochondrial function but does not affect insulin sensitivity or brown adipose tissue in first degree relatives of patients with 
type 2 diabetes. Mol Metab 12, 39-47.

Devries MC, Lowther SA, Glover AW, Hamadeh MJ \& Tarnopolsky MA. (2007). IMCL area density, but not IMCL utilization, is higher in women during moderate-intensity endurance exercise, compared with men. American Journal of Physiology-Regulatory, Integrative and Comparative Physiology 293, R2336-R2342.

Devries MC, Samjoo IA, Hamadeh MJ, McCready C, Raha S, Watt MJ, Steinberg GR \& Tarnopolsky MA. (2013). Endurance Training Modulates Intramyocellular Lipid Compartmentalization and Morphology in Skeletal Muscle of Lean and Obese Women. The Journal of Clinical Endocrinology \& Metabolism 98, 4852-4862.

Gemmink A, Bosma M, Kuijpers HJ, Hoeks J, Schaart G, van Zandvoort MA, Schrauwen P \& Hesselink MK. (2016). Decoration of intramyocellular lipid droplets with PLIN5 modulates fasting-induced insulin resistance and lipotoxicity in humans. Diabetologia 59, 1040-1048.

Gemmink A, Daemen S, Brouwers B, Huntjens PR, Schaart G, Moonen-Kornips E, Jörgensen J, Hoeks J, Schrauwen P \& Hesselink MKC. (2018a). Dissociation of intramyocellular lipid storage and insulin resistance in trained athletes and type 2 diabetes patients; involvement of perilipin 5? J Physiol 596, 857-868.

Gemmink A, Daemen S, Kuijpers HJH, Schaart G, Duimel H, López-Iglesias C, van Zandvoort MAMJ, Knoops K \& Hesselink MKC. (2018b). Super-resolution microscopy localizes perilipin 5 at lipid droplet-mitochondria interaction sites and at lipid droplets juxtaposing to perilipin 2. Biochimica et Biophysica Acta (BBA) - Molecular and Cell Biology of Lipids 1863, 1423-1432.

Gemmink A, Schrauwen P \& Hesselink MKC. (2020). Exercising your fat (metabolism) into shape: a muscle-centred view. Diabetologia 63, 1453-1463.

Goodpaster BH, He J, Watkins S \& Kelley DE. (2001). Skeletal muscle lipid content and insulin resistance: evidence for a paradox in endurance-trained athletes. J Clin Endocrinol Metab 86, 5755-5761.

Goossens GH, Jocken JWE \& Blaak EE. (2021). Sexual dimorphism in cardiometabolic health: the role of adipose tissue, muscle and liver. Nature Reviews Endocrinology 17, 47-66.

He J, Goodpaster BH \& Kelley DE. (2004). Effects of weight loss and physical activity on muscle lipid content and droplet size. Obes Res 12, 761-769.

He J, Watkins S \& Kelley DE. (2001). Skeletal Muscle Lipid Content and Oxidative Enzyme Activity in Relation to Muscle Fiber Type in Type 2 Diabetes and Obesity. Diabetes 50, 817.

Hodge BA, Wen Y, Riley LA, Zhang X, England JH, Harfmann BD, Schroder EA \& Esser KA. (2015). The endogenous molecular clock orchestrates the temporal separation of substrate metabolism in skeletal muscle. Skeletal Muscle 5, 17.

Høeg L, Roepstorff C, Thiele M, Richter EA, Wojtaszewski JFP \& Kiens B. (2009). Higher intramuscular triacylglycerol in women does not impair insulin sensitivity and proximal insulin signaling. J Appl Physiol 107, 824-831.

Høeg LD, Sjøberg KA, Jeppesen J, Jensen TE, Frøsig C, Birk JB, Bisiani B, Hiscock N, Pilegaard H, Wojtaszewski JFP, Richter EA \& Kiens B. (2011). Lipid-Induced Insulin Resistance Affects Women Less Than Men and Is Not Accompanied by Inflammation or Impaired Proximal Insulin Signaling. Diabetes 60, 64 .

Hood DA. (2001). Plasticity in skeletal, cardiac, and smooth muscle - Invited review: Contractile activity-induced mitochondrial biogenesis in skeletal muscle. J Appl Physiol 90, 1137- 
1157.

Horton TJ, Commerford SR, Pagliassotti MJ \& Bessesen DH. (2002). Postprandial leg uptake of triglyceride is greater in women than in men. American Journal of PhysiologyEndocrinology and Metabolism 283, E1192-E1202.

Houtkooper RH \& Vaz FM. (2008). Cardiolipin, the heart of mitochondrial metabolism. Cell Mol Life Sci 65, 2493-2506.

Houzelle A, Jörgensen JA, Schaart G, Daemen S, van Polanen N, Fealy CE, Hesselink MKC, Schrauwen P \& Hoeks J. (2021). Human skeletal muscle mitochondrial dynamics in relation to oxidative capacity and insulin sensitivity. Diabetologia 64, 424-436.

Jevons EFP, Gejl KD, Strauss JA, Ørtenblad N \& Shepherd SO. (2020). Skeletal muscle lipid droplets are resynthesized before being coated with perilipin proteins following prolonged exercise in elite male triathletes. American Journal of PhysiologyEndocrinology and Metabolism 318, E357-E370.

Kahn D, Perreault L, Macias E, Zarini S, Newsom SA, Strauss A, Kerege A, Harrison K, Snell-Bergeon J \& Bergman BC. (2021). Subcellular localisation and composition of intramuscular triacylglycerol influence insulin sensitivity in humans. Diabetologia 64, 168-180.

Koh H-CE, Nielsen J, Saltin B, Holmberg H-C \& Ørtenblad N. (2017). Pronounced limb and fibre type differences in subcellular lipid droplet content and distribution in elite skiers before and after exhaustive exercise. The Journal of physiology 595, 5781-5795.

Li X, Youngren JF, Hyun B, Sakkas GK, Mulligan K, Majumdar S, Masharani UB, Schambelan M \& Goldfine ID. (2008). Technical evaluation of in vivo abdominal fat and IMCL quantification using MRI and MRSI at 3 T. Magn Reson Imaging 26, 188-197.

Li Y, Lee S, Langleite T, Norheim F, Pourteymour S, Jensen J, Stadheim HK, Storås TH, Davanger S, Gulseth HL, Birkeland KI, Drevon CA \& Holen T. (2014). Subsarcolemmal lipid droplet responses to a combined endurance and strength exercise intervention. Physiol Rep 2.

Listenberger LL, Han X, Lewis SE, Cases S, Farese RV, Ory DS \& Schaffer JE. (2003). Triglyceride accumulation protects against fatty acid-induced lipotoxicity. Proceedings of the National Academy of Sciences 100, 3077-3082.

Loizides-Mangold U, Perrin L, Vandereycken B, Betts JA, Walhin J-P, Templeman I, Chanon S, Weger BD, Durand C, Robert M, Paz Montoya J, Moniatte M, Karagounis LG, Johnston JD, Gachon F, Lefai E, Riezman H \& Dibner C. (2017). Lipidomics reveals diurnal lipid oscillations in human skeletal muscle persisting in cellular myotubes cultured in vitro. Proceedings of the National Academy of Sciences, 201705821.

Louche K, Badin P-M, Montastier E, Laurens C, Bourlier V, de Glisezinski I, Thalamas C, Viguerie N, Langin D \& Moro C. (2013). Endurance Exercise Training Up-Regulates Lipolytic Proteins and Reduces Triglyceride Content in Skeletal Muscle of Obese Subjects. The Journal of Clinical Endocrinology \& Metabolism 98, 4863-4871.

Malenfant P, Joanisse DR, Thériault R, Goodpaster BH, Kelley DE \& Simoneau JA. (2001). Fat content in individual muscle fibers of lean and obese subjects. International Journal of Obesity 25, 1316-1321.

Moro C, Galgani JE, Luu L, Pasarica M, Mairal A, Bajpeyi S, Schmitz G, Langin D, Liebisch G \& Smith SR. (2009). Influence of Gender, Obesity, and Muscle Lipase Activity on Intramyocellular Lipids in Sedentary Individuals. The Journal of Clinical Endocrinology \& Metabolism 94, 3440-3447. 
Nielsen J, Christensen AE, Nellemann B \& Christensen B. (2017). Lipid droplet size and location in human skeletal muscle fibers are associated with insulin sensitivity. Am J Physiol Endocrinol Metab 313, E721-E730.

Nielsen J, Mogensen M, Vind BF, Sahlin K, Højlund K, Schrøder HD \& Ørtenblad N. (2010). Increased subsarcolemmal lipids in type 2 diabetes: effect of training on localization of lipids, mitochondria, and glycogen in sedentary human skeletal muscle. American Journal of Physiology-Endocrinology and Metabolism 298, E706-E713.

Nuutila P, Knuuti MJ, Mäki M, Laine H, Ruotsalainen U, Teräs M, Haaparanta M, Solin O \& Yki-Järvinen H. (1995). Gender and Insulin Sensitivity in the Heart and in Skeletal Muscles: Studies Using Positron Emission Tomography. Diabetes 44, 31.

Perreault L, Bergman BC, Hunerdosse DM \& Eckel RH. (2010). Altered Intramuscular Lipid Metabolism Relates to Diminished Insulin Action in Men, but Not Women, in Progression to Diabetes. Obesity 18, 2093-2100.

Perreault L, Newsom SA, Strauss A, Kerege A, Kahn DE, Harrison KA, Snell-Bergeon JK, Nemkov T, D'Alessandro A, Jackman MR, MacLean PS \& Bergman BC. (2018). Intracellular localization of diacylglycerols and sphingolipids influences insulin sensitivity and mitochondrial function in human skeletal muscle. JCI Insight 3, e96805.

Peters SJ, Samjoo IA, Devries MC, Stevic I, Robertshaw HA \& Tarnopolsky MA. (2012). Perilipin family (PLIN) proteins in human skeletal muscle: the effect of sex, obesity, and endurance training. Appl Physiol Nutr Metab 37, 724-735.

Pourteymour S, Lee S, Langleite TM, Eckardt K, Hjorth M, Bindesbøll C, Dalen KT, Birkeland KI, Drevon CA, Holen T \& Norheim F. (2015). Perilipin 4 in human skeletal muscle: localization and effect of physical activity. Physiological Reports 3, e12481.

Rambold AS, Cohen S \& Lippincott-Schwartz J. (2015). Fatty acid trafficking in starved cells: regulation by lipid droplet lipolysis, autophagy, and mitochondrial fusion dynamics. Dev Cell 32, 678-692.

Remie CME, Moonen MPB, Roumans KHM, Nascimento EBM, Gemmink A, Havekes B, Schaart G, Kornips E, Joris PJ, Schrauwen-Hinderling VB, Hoeks J, Kersten S, Hesselink MKC, Phielix E, Lichtenbelt WDvM \& Schrauwen P. (2021). Metabolic responses to mild cold acclimation in type 2 diabetes patients. Nature Communications 12, 1516.

Rhee EP, Cheng S, Larson MG, Walford GA, Lewis GD, McCabe E, Yang E, Farrell L, Fox CS, O’Donnell CJ, Carr SA, Vasan RS, Florez JC, Clish CB, Wang TJ \& Gerszten RE. (2011). Lipid profiling identifies a triacylglycerol signature of insulin resistance and improves diabetes prediction in humans. The Journal of Clinical Investigation 121, 1402-1411.

Roepstorff C, Steffensen CH, Madsen M, Stallknecht B, Kanstrup I-L, Richter EA \& Kiens B. (2002). Gender differences in substrate utilization during submaximal exercise in endurance-trained subjects. American Journal of Physiology-Endocrinology and Metabolism 282, E435-E447.

Samjoo IA, Safdar A, Hamadeh MJ, Glover AW, Mocellin NJ, Santana J, Little JP, Steinberg GR, Raha S \& Tarnopolsky MA. (2013). Markers of skeletal muscle mitochondrial function and lipid accumulation are moderately associated with the homeostasis model assessment index of insulin resistance in obese men. PLoS One 8, e66322.

Sattar N. (2013). Gender aspects in type 2 diabetes mellitus and cardiometabolic risk. Best Practice \& Research Clinical Endocrinology \& Metabolism 27, 501-507. 


\section{Chapter 8}

Schenk S \& Horowitz JF. (2007). Acute exercise increases triglyceride synthesis in skeletal muscle and prevents fatty acid-induced insulin resistance. J Clin Invest 117, 16901698.

Schwab U, Seppänen-Laakso T, Yetukuri L, Ågren J, Kolehmainen M, Laaksonen DE, Ruskeepää A-L, Gylling H, Uusitupa M, Orešič M \& for the GSG. (2008). Triacylglycerol Fatty Acid Composition in Diet-Induced Weight Loss in Subjects with Abnormal Glucose Metabolism - the GENOBIN Study. PLOS ONE 3, e2630.

Shaw CS, Jones DA \& Wagenmakers AJM. (2008). Network distribution of mitochondria and lipid droplets in human muscle fibres. Histochemistry and Cell Biology 129, 65-72.

Shaw CS, Shepherd SO, Wagenmakers AJM, Hansen D, Dendale P \& Loon LJCv. (2012). Prolonged exercise training increases intramuscular lipid content and perilipin 2 expression in type I muscle fibers of patients with type 2 diabetes. American Journal of Physiology-Endocrinology and Metabolism 303, E1158-E1165.

Shepherd SO, Cocks M, Tipton KD, Ranasinghe AM, Barker TA, Burniston JG, Wagenmakers AJ \& Shaw CS. (2013). Sprint interval and traditional endurance training increase net intramuscular triglyceride breakdown and expression of perilipin 2 and 5. J Physiol 591, 657-675.

Shepherd SO, Cocks M, Tipton KD, Ranasinghe AM, Barker TA, Burniston JG, Wagenmakers AJM \& Shaw CS. (2012). Preferential utilization of perilipin 2-associated intramuscular triglycerides during $1 \mathrm{~h}$ of moderate-intensity endurance-type exercise. Experimental Physiology 97, 970-980.

Shepherd SO, Strauss JA, Wang Q, Dube JJ, Goodpaster B, Mashek DG \& Chow LS. (2017). Training alters the distribution of perilipin proteins in muscle following acute free fatty acid exposure. The Journal of physiology 595, 5587-5601.

Sidossis LS, Wolfe RR \& Coggan AR. (1998). Regulation of fatty acid oxidation in untrained vs. trained men during exercise. American Journal of Physiology-Endocrinology and Metabolism 274, E510-E515.

Skovbro M, Baranowski M, Skov-Jensen C, Flint A, Dela F, Gorski J \& Helge JW. (2008). Human skeletal muscle ceramide content is not a major factor in muscle insulin sensitivity. Diabetologia 51, 1253-1260.

Steffensen CH, Roepstorff C, Madsen M \& Kiens B. (2002). Myocellular triacylglycerol breakdown in females but not in males during exercise. American Journal of Physiology-Endocrinology and Metabolism 282, E634-E642.

Straczkowski M, Kowalska I, Nikolajuk A, Dzienis-Straczkowska S, Kinalska I, Baranowski M, Zendzian-Piotrowska M, Brzezinska Z \& Gorski J. (2004). Relationship Between Insulin Sensitivity and Sphingomyelin Signaling Pathway in Human Skeletal Muscle. Diabetes 53, 1215-1221.

Szendroedi J, Yoshimura T, Phielix E, Koliaki C, Marcucci M, Zhang D, Jelenik T, Müller J, Herder C, Nowotny P, Shulman GI \& Roden M. (2014). Role of diacylglycerol activation of PKC $\theta$ in lipid-induced muscle insulin resistance in humans. Proceedings of the National Academy of Sciences 111, 9597-9602.

Tarnopolsky LJ, MacDougall JD, Atkinson SA, Tarnopolsky MA \& Sutton JR. (1990). Gender differences in substrate for endurance exercise. J Appl Physiol (1985) 68, 302-308.

Tarnopolsky MA, Rennie CD, Robertshaw HA, Fedak-Tarnopolsky SN, Devries MC \& Hamadeh MJ. (2007). Influence of endurance exercise training and sex on intramyocellular lipid and mitochondrial ultrastructure, substrate use, and mitochondrial enzyme 
activity. American Journal of Physiology-Regulatory, Integrative and Comparative Physiology 292, R1271-R1278.

Timmers S, de Ligt M, Phielix E, van de Weijer T, Hansen J, Moonen-Kornips E, Schaart G, Kunz I, Hesselink MK, Schrauwen-Hinderling VB \& Schrauwen P. (2016). Resveratrol as Add-on Therapy in Subjects With Well-Controlled Type 2 Diabetes: A Randomized Controlled Trial. Diabetes Care 39, 2211-2217.

Timmers S, Konings E, Bilet L, Houtkooper RH, van de Weijer T, Goossens GH, Hoeks J, van der Krieken S, Ryu D, Kersten S, Moonen-Kornips E, Hesselink MKC, Kunz I, Schrauwen-Hinderling VB, Blaak E, Auwerx J \& Schrauwen P. (2011). Calorie restriction-like effects of 30 days of resveratrol supplementation on energy metabolism and metabolic profile in obese humans. Cell Metab 14, 612-622.

van Loon LJ, Koopman R, Stegen JH, Wagenmakers AJ, Keizer HA \& Saris WH. (2003). Intramyocellular lipids form an important substrate source during moderate intensity exercise in endurance-trained males in a fasted state. J Physiol 553, 611-625.

Vistisen B, Hellgren LI, Vadset T, Scheede-Bergdahl C, Helge JW, Dela F \& Stallknecht B. (2008). Effect of gender on lipid-induced insulin resistance in obese subjects. Eur J Endocrinol 158, 61-68.

Wefers J, van Moorsel D, Hansen J, Connell NJ, Havekes B, Hoeks J, van Marken Lichtenbelt WD, Duez H, Phielix E, Kalsbeek A, Boekschoten MV, Hooiveld GJ, Hesselink MKC, Kersten S, Staels B, Scheer FAJL \& Schrauwen P. (2018). Circadian misalignment induces fatty acid metabolism gene profiles and compromises insulin sensitivity in human skeletal muscle. Proceedings of the National Academy of Sciences 115, 77897794.

Wilson JM, Loenneke JP, Jo E, Wilson GJ, Zourdos MC \& Kim J-S. (2012). The Effects of Endurance, Strength, and Power Training on Muscle Fiber Type Shifting. The Journal of Strength \& Conditioning Research 26.

Wolins NE, Brasaemle DL \& Bickel PE. (2006). A proposed model of fat packaging by exchangeable lipid droplet proteins. FEBS Letters 580, 5484-5491. 


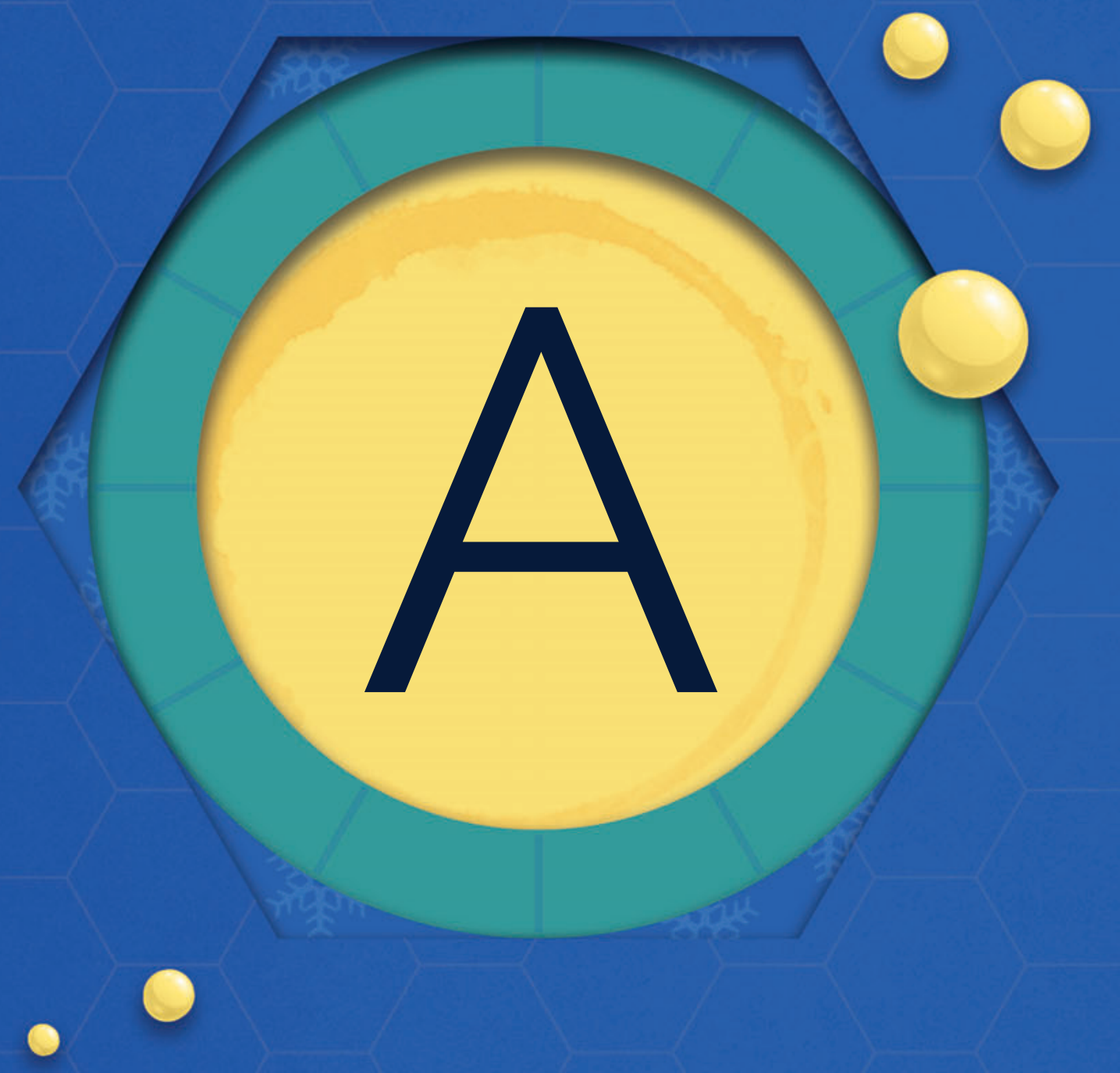




\section{Appendix}

Impact paragraph

Samenvatting

Dankwoord

List of publications

About the author 



\section{Impact paragraph}

\section{What is the aim of the thesis and what are the main results and conclusions?}

Diabetes effects the quality of life of more than 1 million people in the Netherlands and is amongst the top 3 diseases with the highest disease burden. Ninety percent of the people with diabetes suffers from type 2 diabetes, who have high blood glucose (sugar) levels and a decreased functioning of the hormone insulin. Insulin regulates the uptake of blood sugar into the tissues after a meal, to make sure that the blood glucose levels will not be too high. When tissues do not respond properly to insulin, which is observed in type 2 diabetes, the tissues in the body take up less glucose and it remains in the blood. This can lead to high blood sugar levels. The muscle is an important tissue in this process as it takes up about $80 \%$ of all the blood sugar after a meal. When the muscle becomes less responsive to insulin, known as insulin resistance, this is one of the first signs of developing type 2 diabetes.

How the muscle becomes less responsive to insulin (insulin resistance) is not yet known. Fat accumulation in the muscle can play a role and interfere with the signals of insulin to the muscle. Fat, or often in science called lipid, is mostly stored in fat tissue. However, it can also be stored on other places such as in the liver, muscle or heart. People that are obese or have type 2 diabetes store large amounts of fat in the muscle and this may lead to insulin resistance. Interestingly, people who run marathons or cycle long distances also store large amounts of fat in their muscle, but they remain very responsive to insulin (also called insulin sensitivity). Perhaps these active people use their muscle fat more efficiently or store it in a different way than the muscle fat in patients with type 2 diabetes. Muscle fat is normally stored in the form of lipid droplets. If we look more in detail, we see indeed that active people store their muscle fat in smaller and more lipid droplets, especially in the red muscle fibres and a protein called PLIN5 is more present. Perhaps this could explain why these active people have more muscle fat but still remain responsive to insulin, and people with type 2 diabetes become insulin resistant. 


\section{Appendix}

In chapter 2 we looked at scientific literature to see how diet and exercising can affect muscle fat. We have seen that muscle fat is dynamic and adapts to the type of diet and exercise of a person. When someone eats more than he or she uses, muscle fat increases and when a person reduces his calories, the muscle fat decreases again. During a single bout of exercise muscle fat can be used as fuel to provide energy. When someone trains for a longer time, the total muscle fat increases. The muscle adapts to the new lifestyle and an increase of muscle fat is part of this. This suggests that if the muscle fat is used and stays dynamic this may not lead to unhealthy consequences. However, a lot of questions still remain unanswered. How does muscle fat stay dynamic? When is muscle fat healthy? Could we modulate the muscle fat of patients with type 2 diabetes to become more dynamic and therefore make them healthier?

To make patients with type 2 diabetes healthier, many different risk factors have been studied and treatments have been proposed. A new possible risk factor is the changes in day-night rhythm. People who work as shift workers (so work during the night and sleep during the day for a short period of time and then change back to their 'normal' rhythm) show that the muscle becomes insulin resistant and these people are more at risk of becoming obese or develop type 2 diabetes. In chapter 4 we wanted to see if the muscle fat was also altered. When shift work was mimicked in the lab, we found a decrease in insulin sensitivity, but no changes in the muscle fat. Perhaps the experiment was too short and maybe muscle fat will change on the longer term. The participants were also young and healthy and they had relative low levels of muscle fat. With specialized techniques we looked further at different kind of lipids and we discovered that some lipid species were changed during shift work. These species might be interesting to look at in the future and if they have influence on insulin sensitivity.

In the other chapters we examined certain treatment options to improve the responsiveness of insulin (insulin sensitivity) and see if there were also changes in muscle fat, which could give more information about the relation between muscle fat and insulin sensitivity. In chapter 3 , humans were exposed to short-term cold 
exposure and this led to increased energy expenditure and fat oxidation. To our surprise, the lipid levels in the blood did not drop, which was earlier seen in animal studies. Perhaps it works different in humans and the body keeps the blood fat levels high to provide energy to stay warm. If the cold exposure would have been bit longer or if we would have activated shivering (which was not the case here, despite the cold environment), we could have observed changes in lipid levels in the blood. Shivering activates the muscle and this may lead to more uptake of lipids by the muscle and therefore lowers blood lipid levels.

In chapter 5, we studied the effect of the nutritional supplement resveratrol. Resveratrol is naturally present in grapes and peanuts and boosts energy expenditure. Some studies showed that resveratrol also increased muscle fat without causing insulin resistance. We found that resveratrol modulated the muscle fat similar to what we have seen in active people: smaller lipid droplets, stored especially in red muscle fibres with the presence of the PLIN5 protein. Resveratrol can be a possible candidate to modulate the muscle fat to a healthier way of storage. More studies are needed to explore this, especially on the longer term.

In chapter 6, we looked more in detail what happens to the muscle fat when you exercise. Thus, we examined muscle fat before exercise, just after exercise and during recovery and investigated how it changes. In addition, does it matter whether you stay fasted during exercises or consume sugars? To study this, one group was only allowed to drink water during this study (the fasted group), while the other drank a sugar drink. We found that in the group with the sugar drink, not much happened with the muscle fat. But in the group that drank water, the muscle fat was used during exercise and during recovery it increased again. During recovery, the muscle fat in the water group was much higher than in the sugar group. The muscle fat was especially stored in the red muscle fibres and in the presence of PLIN5. This shows that drinking calories (sugar) can affect the use of your muscle fat during exercise.

If we combine the findings from this thesis with other scientific literature, we can consider that fat storage in the muscle is not necessarily bad. As long as you store it 


\section{Appendix}

in a healthy way and use it. It seems that it is better to store your muscle fat in many small lipid droplets (not a few big ones), especially in muscle fibres that oxidize these lipids (red muscle fibres) and preferably with the protein PLIN5 on these lipid droplets. It also matters what kind of lipid you store in your muscle cells. If your day-night rhythm is disrupted some specific lipid species change, but more research is needed how these lipid species matter and if they can have an effect on insulin sensitivity.

\section{What is the potential contribution of these results to science and society?}

This thesis contributes to the knowledge of the metabolism of muscle fat and how it changes in certain circumstances. This broadens our understanding of the behaviour of lipid droplets inside the muscle that form the total fat storage in the muscle. We looked at many details of the muscle fat, like the number and size of lipid droplets, the involvement of the PLIN5 protein and where the muscle fat is located. All these different features can react differently upon diet or exercise and are therefore important to study. Our results show that it is important to not only look at total muscle fat, but to look also at more details to better understand muscle fat metabolism and its health consequences. The amount of muscle fat cannot fully explain whether a muscle is metabolically healthy (here: insulin-sensitive) or not.

As it is still not known how tissues become insulin resistant, so more studies are needed to understand the development of type 2 diabetes. When it is more evident how insulin resistance evolves, this knowledge can be used to prevent the development of type 2 diabetes. Fat storage is associated with obesity, which is an important risk factor for type 2 diabetes. Unhealthy fat storage in the muscle can facilitate the development of insulin resistance. If this relation is better understood, the development of insulin resistance can be prevented and therefore prevent new cases of type 2 diabetes. 
The knowledge in this thesis can also be interesting for new studies to modulate muscle fat. For example, we observed that resveratrol can modulate muscle fat into a healthier way of storage. If we could develop strategies to help patients with type 2 diabetes to make their muscle fat more dynamic (and healthier) this might reduce their symptoms and improve the quality of life. Alternatively, specific markers could be developed in the future to identify which person has a healthy muscle fat storage.

In this thesis we used microscopy to study muscle fat. For this, small muscle biopsies were taken from human volunteers and we looked at lipid droplets within the muscle pieces. Microscopy is a powerful tool to obtain information on fat storage within a single muscle cell. Maastricht University has a specialized facility where different kinds of microscopes can be used. With the microscope we are able to look at more details such as number and size of lipid droplets, location of these lipid droplets and examine the PLIN5 protein. By using both human studies and microscopy techniques, we combine the knowledge about what happens in the muscle with the knowledge what happens in the human body. This makes it easier to translate findings to future clinical studies with patients. Furthermore, the microscopy facility enables us to develop more techniques to study the dynamics of muscle fat as we proposed in chapter 7. This gives us more knowledge on the cell level, to better understand how fat is stored in the muscle.

\section{To whom would these results be interesting?}

The results of this thesis are mainly interesting for researchers. At first for researchers that also study muscle fat metabolism and/or the development of type 2 diabetes. This thesis can provide another piece of the puzzle for the field of muscle fat and insulin sensitivity. Furthermore, fat is not only stored in the muscle but also in other tissues and is associated with many other diseases such as heart diseases, Alzheimer's and cancer. Researchers in this field can also benefit from our findings. Lipid droplets are not only studied in human tissues but also in totally different disciplines. For example, lipid droplets are also important for foetal development, for viruses to infect people or to study the use of lipid droplets in biofuel. Studying lipid droplets itself 


\section{Appendix}

can be beneficial for researchers in many different fields.

Microscopy is a growing interest for many researchers and more groups are starting to use the techniques. Researchers that are interested in microscopy would be interested in this thesis to use for example the same techniques or for those who want to cooperate with our facilities.

Most studies performed in this thesis are done to gain more knowledge. However, this knowledge can be relevant for research that is directly involving patients. If we understood how muscle fat works and can be modulated, this can be relevant for patients to improve their quality of life.

The results of this thesis are published in scientific papers, or will be in the future, which are available to read for anyone who is interested. These results are published in journals, online and via social media. Many results have been presented and discussed in national and international conferences with other researchers, patient organizations or representatives from compagnies. In the end, the knowledge can also be used for educational purposes for students or other audiences. 
Impact paragraph 



\section{Samenvatting}

Meer dan één miljoen mensen in Nederland hebben diabetes, waarvan $90 \%$ type 2 diabetes. Patiënten met type 2 diabetes worden gekenmerkt door insulineresistentie. Bij insulineresistentie reageren de weefsels in het lichaam minder op insuline waardoor minder glucose wordt opgenomen. Dit kan leiden tot hoge glucosewaarden in het bloed. De spier neemt na een maaltijd ongeveer $80 \%$ van de totale glucose op uit het bloed en insulineresistentie in de spier is dan ook een van de eerste kenmerken van het ontwikkelen van type 2 diabetes. Hoe insulineresistentie ontstaat is nog niet bekend. Mogelijk speelt overmatige vetopslag een belangrijke rol in het ontstaan van insulineresistentie van de spier.

Vet (lipiden) worden in de spiercel (myocel) opgeslagen als zogenaamde intramyocellulaire lipiden (IMCL), onder andere in de vorm van lipide druppels (LDs). Een toename van IMCL wordt geassocieerd met een toename in insulineresistentie. Duuratleten hebben, paradoxaal genoeg, ook grote hoeveelheden IMCL, maar zijn insulinegevoelig. Dit fenomeen is bekend als 'de atleten-paradox'. Lipiden in de spier interfereren dus niet altijd met insulinegevoeligheid. In duuratleten ondergaan LDs een constante cyclus van opslag en verbruik, waarbij interactie plaatsvindt met andere organellen zoals mitochondriën. LDs worden tegenwoordig gezien als dynamische organellen die lipiden opslaan of vrijmaken afhankelijk van de voeding of de energieproductie die nodig is. Het behouden van deze dynamiek is belangrijk om insulineresistentie en metabole ziektes te voorkomen. Om de aspecten van LD dynamiek te bestuderen, richt onderzoek zich niet alleen op de totale vetopslag in de spier, maar ook op veranderingen in LD morfologie (aantal en grootte), locatie van de LDs in de cel en de aanwezigheid van bepaalde eiwitten op de LDs.

LDs bestaan uit een neutrale lipide kern van voornamelijk triglyceriden en cholesterolesters omringt door een fosfolipidenmembraan. Op dit membraan bevinden zich ook veel verschillende eiwitten, waaronder lipasen en perilipine 5 (PLIN5). PLIN5 komt voor in de spier en speelt een rol bij de opslag van lipiden in LDs en het vrijmaken van vetzuren uit LDs voor oxidatie. Een goede balans tussen 


\section{Appendix}

de opslag en het vrijmaken van vetzuren is belangrijk om lipotoxiciteit te voorkomen. Lipotoxiciteit is de aanwezigheid van bioactieve lipiden in het cytoplasma die het functioneren van de cel belemmeren. Mogelijk kunnen deze bioactieve lipiden de insulinesignalering hinderen wat kan leiden tot insuline-resistentie. LDs en eiwitten die een rol spelen in vetmetabolisme spelen een grote rol om lipotoxiciteit te voorkomen. Vetopslag gekenmerkt door een hoog aantal LDs wordt geassocieerd met insuline-gevoeligheid, terwijl grote LDs geassocieerd zijn met insulineresistentie. Individuen met een hoge insulinegevoeligheid hebben meer PLIN5 in hun spieren en meer LDs met PLIN5 op het membraan. Hieruit blijkt dat de morfologie van LDs en de aanwezigheid van PLIN5 een belangrijke rol kunnen spelen in insulinegevoeligheid.

De spier bestaat uit type I en type II vezels. Type I vezels hebben een hoge oxidatieve capaciteit, bevatten veel mitochondriën en hebben meer IMCL dan type II vezels. Type II vezels hebben vooral een hoge anaerobe capaciteit en gebruiken voornamelijk koolhydraten. Daarom is het belangrijk om beide vezeltypen in acht te nemen wanneer er gekeken wordt naar IMCL. Binnen een spiercel bevinden de LDs zich tussen de myofibrillen (intermyofibrillaire (IMF) LDs) en vlak onder de celmembraan (subsarcolemmale (SS) LDs). Er wordt gedacht dat IMF-LDs energie kunnen leveren aan de IMF- mitochondriën die belangrijk zijn voor het produceren van ATP voor spiercontractie. SS-LDs bevinden zich vlak bij het vasculaire systeem en worden daarom beïnvloed door hoge lipide waarden in het bloed die worden opgenomen door de spier. De insulinereceptoren bevinden zich in de celmembraan en daarom wordt gedacht dat vooral SS-LDs negatieve invloed kunnen hebben op de insulinesignalering. Patiënten met type 2 diabetes hebben meer SS-LDs, met name in type II spiervezels, en SS-LDs worden dan ook geassocieerd met insulineresistentie.

Hoofdstuk 2 bevat een literatuuroverzicht van de huidige wetenschappelijke literatuur over de invloed van voeding en fysieke activiteit op IMCL en de relatie tot insulinegevoeligheid. Voeding en inspanning hebben beide invloed op LD dynamiek. Een hoge vetinname, en daarbij verhoogde triglyceridewaarden en vrije vetzuren in het bloed, zorgt voor een verhoogde vetopname in de spier en daarmee een toename in IMCL. IMCL neemt weer af tijdens calorierestrictie, 
hoewel langdurig vasten leidt tot een toename in IMCL (waarschijnlijk door op den duur verhoogde vetzuren in het bloed). IMCL wordt ook gebruikt als energiebron tijdens acute inspanning. Duurtraining zorgt op langer termijn voor een toename in IMCL, waarbij IMCL een belangrijke energiebron is. IMCL is dus een dynamische verzameling van LDs die zich aanpassen aan energieaanbod en -vraag. Door middel van onder andere fluorescentiemicroscopie kunnen aspecten van LD dynamiek geanalyseerd worden. Deze methodiek wordt ook toegepast in hoofdstukken 4 $\mathbf{t} / \mathbf{m} \mathbf{6}$. Het doel van dit proefschrift is om de rol van LD dynamiek te besturen in relatie tot insulinegevoeligheid in verschillende humane interventiestudies. Daarom hebben we lipiden en kenmerken van LD dynamiek bestudeerd na blootstelling aan kou (hoofdstuk 3), verstoord dag-nacht ritme (hoofdstuk 4), inname van het voedingssupplement resveratrol (hoofdstuk 5) en acute inspanning (hoofdstuk 6).

In hoofdstuk 3 hebben we gekeken naar de invloed op kou op triglyceridengehalte in het bloed. Insulineresistente individuen hebben hoge triglyceridenwaarden in hun bloed en daarmee meer risico op het ontwikkelen van hart- en vaatziektes. In deze studie zorgde acute kou voor een verhoogd energiegebruik met name door een verhoogde vetoxidatie, maar dit leidde niet tot een verhoogde triglycerideklaring. In dierenstudies wordt bruin vetweefsel geactiveerd door kou en vervolgens de triglyceride klaring verhoogd. In mensen is het bruin vetweefsel wellicht niet voldoende om het triglyceridegehalte in het bloed te doen verminderen. In deze studie werd gebruik gemaakt van kou geïnduceerde thermogenese zonder dat de proefpersonen aan het rillen waren (spieractivatie). Blootstelling tot langdurige kou of het toestaan van rillen zouden wellicht het triglyceridengehalten in het bloed kunnen doen dalen in mensen.

In hoofdstuk 4 zijn we verder gaan kijken naar de relatie tussen insulinegevoeligheid en IMCL. In eerdere studies hebben we gezien dat een verstoord slaap-waak ritme leidt tot een verminderde insulinegevoeligheid. Dit komt bijvoorbeeld voor bij mensen die in ploegdiensten werken en daarbij ook nachtdiensten draaien. Het doel van dit hoofdstuk was om te achterhalen of deze verminderde insulinegevoeligheid te verklaren is door veranderingen van lipiden in de spier. Echter zagen we geen 


\section{Appendix}

veranderingen in IMCL, LD morfologie of locatie van LDs na een verstoring van slaapwaak ritme. Met behulp van lipidomics technieken is er gekeken naar verschillende soorten lipiden en daaruit bleek dat enkele type lipiden (cardiolipine en zeer lange verzuren) waren veranderd door een verstoorde slaap-waak ritme. Deze type lipiden zijn interessant om verder te onderzoeken en hun relatie met insuline-gevoeligheid te bestuderen.

Resveratrol is een polyfenol dat van nature voorkomt in onder andere druiven en pinda's. In eerdere studies is gebleken dat resveratrol de mitochondriële functie verbeterd en dat dit gaat gepaard met een toename in IMCL en afname in levervet. Op welke manier resveratrol voor deze effecten zorgt is nog onbekend. Deze veranderingen in IMCL zijn daarentegen vergelijkbaar met mensen die gaan trainen. Resveratrol is daardoor een interessant voedingssupplement dat IMCL beïnvloed op een manier dat wordt geassocieerd met gezondheidswinst. In hoofdstuk 5 wordt de hypothese getest of de toename in IMCL na resveratrol-inname (in de afwezigheid van een daling in insulinegevoeligheid) gepaard gaat met veranderingen in LD morfologie die overeenkomen met de LD morfologie van duurgetrainde atleten. Het doel van dit hoofdstuk was om de verhoogde vetopslag na 30 dagen resveratrolinname te bestuderen in individuen met verminderde stofwisseling en of deze vetopslag in de spier lijkt op een 'gezonde opslag'. In deze studie zagen we bij individuen die een toename lieten zien in IMCL, dat dit vooral werd opgeslagen in type I vezels en in LDs met PLIN5. Dit is vergelijkbaar met de vetopslag die in atleten wordt gezien, en een toename in IMCL door resveratrol hoeft daarom niet negatief te zijn voor de gezondheid.

In hoofdstuk 6 hebben we uitgebreider gekeken naar de invloed van acute inspanning op IMCL. Hierbij hebben we LDs geanalyseerd bij rust (voor het sporten), direct na inspanning en 4 uur na het sporten (herstel) in ongetrainde jonge mannen. Daarnaast hebben we de invloed van suikerinname tijdens het sporten bestudeerd door één groep te laten fietsen en herstellen in gevaste toestand (alleen het drinken van water was toegestaan) en de andere groep een glucosedrankje te laten drinken tijdens het hele experiment. Na 2 uur fietsen in gevaste toestand nam de vetopslag af in 
de SS-regio en niet in de IMF-regio. Dit duidt op een rol van SS LDs tijdens acute inspanning in gevaste toestand. Tijdens het 4-uur durende herstel nam de IMCL weer toe in de gevaste toestand, met name in type I vezels, SS LDs en LDs met PLIN5. Vier uur na het fietsen was IMCL hoger in gevaste toestand dan wanneer glucose werd ingenomen. De invloed van inspanning op IMCL lijkt dus afhankelijk van de gevaste toestand, dat gepaard gaat met hogere vetzuurniveaus in het bloed en hogere vetverbranding.

Zoals boven benoemd, hebben we in dit proefschrift geprobeerd om de dynamiek van de LDs in kaart te brengen door kenmerken van dynamiek voor en na een interventie te meten in mensen. Deze kenmerken worden echter op een tijdspunt gemeten en dynamiek zou nog beter in kaart gebracht kunnen worden door LDs te bestuderen gedurende een bepaalde tijdsduur. In hoofdstuk 7 beschrijven we een nieuwe techniek met behulp van levende cel-microscopie (en fluorescentiemicroscopie) om LD dynamiek te bestuderen in spiercellen aan de hand van twee verschillende fluorescent gelabelde vetzuren. Allereerst wordt een (groen) gelabelde vetzuur toegevoegd om de eerste LDs te vormen. Om vervolgens te bestuderen hoe cellen omgaan met nieuwe vetzuren wordt na deze incubatie een ander gelabeld vetzuur toegevoegd (rood label) om te kijken waar deze nieuwe vetzuren terecht komen (in nieuwgevormde druppels of bestaande druppels). In hoofdstuk 7 worden de stappen beschreven hoe deze methode is ontwikkeld om deze in de toekomst te gebruiken voor verdere onderzoeken. We verwachten dat spiercellen van insulinegevoelige donoren in staat zijn om veel nieuwe LDs te vormen. Spiercellen van insulineresistente donoren zullen grotere en meer statische LDs vormen. De eerste resultaten in een klein aantal donoren laten zien dat spiercellen van insulinegevoelige donoren sneller en meer vetzuren opnemen dan spiercellen van insulineresistente donoren en dat dit vooral resulteert in meer LDs (en geen grotere LDs). In de toekomst kan de methode gebruikt worden om de dynamiek te bestuderen in spiercellen van een grotere groep insulinegevoelige en insulineresistente donoren. Hierbij kan niet alleen worden gekeken waar de vetzuren worden opgeslagen, maar ook wat er gebeurt met de gelabelde vetzuren als lipolyse of vetoxidatie wordt gestimuleerd. Hiermee hopen 


\section{Appendix}

we het mechanisme beter te kunnen begrijpen hoe LD dynamiek invloed heeft op insulinegevoeligheid.

Het doel van het proefschrift was om meer inzicht te krijgen in de dynamiek van IMCL in relatie tot insulinegevoeligheid voor en na verschillende humane interventies. Met behulp van microscopische technieken hebben we niet alleen de totale vetopslag in de spier bestudeerd, maar ook de details van de LDs, waaronder aantal, grootte, locatie en de aanwezigheid van PLIN5 onder invloed van verschillende interventies. Hiermee willen we de LD dynamiek beter in kaart brengen. In hoofdstuk 5 en $\mathbf{6}$ hebben we gezien dat een toename in IMCL na resveratrol-inname of na acute inspanning zich kenmerkt in een hoger aantal LDs, opslag in type I vezels met PLIN5. Dit komt overeen met de manier waarop atleten vet opslaan in de spier. Dit suggereert dat we met behulp van interventies (bijvoorbeeld resveratrol-supplementen of inspanning) de vorm van vetopslag in de spier kunnen veranderen. Het is nog niet bekend hoe insulineresistentie in de spier exact ontstaat en welke rol lipiden daarin een rol spelen. Daarom is het belangrijk om veel aspecten van vetopslag te bestuderen met name vanuit een humaan perspectief. Als het ontstaan van insulineresistentie beter wordt begrepen, dan kunnen therapieën ontwikkeld worden om het spiervet te moduleren en het ontstaan van insulineresistentie te voorkomen/behandelen. Daarmee kunnen nieuwe gevallen van type 2 diabetes voorkomen worden en/of de kwaliteit van leven van de patiënt met type 2 diabetes verbeterd worden. 
Samenvatting

A 



\section{Dankwoord}

Hoera, mijn proefschrift is af! Ik kijk terug op een leerzame periode, waarin ik mij niet alleen als wetenschapper maar ook op persoonlijk vlak heb ontwikkeld. Zoals iedere promovendus heb ik pieken en dalen gekend. Maar dit boekje zal er niet zijn geweest zonder de steun van vele mensen, binnen en buiten de wetenschap.

Ten eerste wil ik mijn promotieteam bedanken, dat ik de mogelijk kreeg om dit promotietraject bij jullie te kunnen doorlopen. Ik heb ontzettend veel van jullie geleerd en bedankt dat ik altijd op jullie kon rekenen. Matthijs, ik kan mij geen betere begeleider wensen. Hoewel ik soms even moest wachten op je feedback, was die altijd waardevol en eerlijk. Bedankt voor al je hulp en geduld de afgelopen jaren. Ik kon altijd bij je terecht met vragen over werk en privé. Ook bedankt voor de mogelijkheid en vertrouwen om het beste uit mij zelf te halen en mij verder te ontwikkelen. Patrick, je kritische blik op ons werk was altijd waardevol. Je passie voor het vak en de groep is mooi om te zien. Bedankt voor al je hulp en steun, vooral tijdens de laatste weken van het afronden van mijn proefschrift. Jouw inzet en betrokkenheid waardeer ik enorm.

Anne en Sabine, ik mocht bij jullie aansluiten bij het 'Lipid droplet team'. Bedankt voor al jullie kennis en vaardigheden. Jullie stonden altijd open voor het beantwoorden van vragen of feedback te geven. Sabine ik wens je veel succes in de VS. Ik hoop dat je daar ook mooie reizen kunt maken. Anne, bedankt voor al je steun en begeleiding. Je was van het begin tot het einde betrokken bij mijn promotie en superfijn dat je mijn copromotor bent. Ik wens je veel succes met je verdere wetenschappelijke carrière, ik denk dat je ver zult komen!

Verder wil ik de rest van de DMRG board, Vera, Esther, Mijke, Joris en Bas bedanken voor jullie steun, vertrouwen en begeleiding de afgelopen jaren. Bedankt voor alle kritische vragen en feedback tijdens de meetings. Désirée en Yolanda, bedankt voor jullie hulp en betrokkenheid.

I like to thank the assessment committee, Prof. Dr. Ronit Shiri-Sverdlov, Prof. Dr. Marc A.M.J. van Zandvoort, Prof. Dr. Ir. Jaap Keijer, Dr. Sam O. Shepherd and Dr. 


\section{Appendix}

Ruth C. R. Meex, for taking the time to read and assess my dissertation and being part of my defence committee.

Lotte en Froukje, ik zal mijn twee Belgen op de kamer missen! Jullie waren hele fijne kamergenoten. Het was rustig als er hard gewerkt moest worden, maar er was zeker ook tijd om een fles wijn open te trekken of ergens een cocktail te gaan drinken. Als mijn PhD even zwaar was, waren er jullie er voor mij. Bedankt voor jullie steun en gezelligheid tijdens mijn PhD en superfijn dat jullie mijn paranimfen willen zijn!

Gert en Esther, bedankt voor alle hulp in het lab. Zonder jullie hulp zouden niet al die spierbiopten zijn gesneden en gekleurd. Jullie zorgden altijd voor een gezellige sfeer en waren samen met Johanna leuke buren! Gert, het was altijd gezellig als jij bij onze meetings aanwezig was en ik zal onze gesprekken over skiën en zingen missen!

Joris en Johanna, door jullie heb ik de passie van oxygraaf metingen gekregen. Joris, jouw kritische blik op onze data was erg waardevol en het was fijn om gedetailleerd door alle Excel sheets heen te gaan. Johanna, bedankt voor alle praktische hulp en gezelligheid in het lab, zelfs in de koude kamer (brrr). Dat geldt ook voor de rest van het oxygraaf-team. Bedankt dat we elkaar zo hielpen, steunden en konden invallen waar nodig.

Verder wil ik alle mensen van het Microscopy CORE Lab bedanken. Jullie hebben mij de mogelijkheden gegeven om de wereld van microscopie te ontdekken. Vooral Helma en Kèvin, bedankt voor al jullie hulp achter de STED en de Corrsight en het beantwoorden van al mijn vragen.

Verder wil ik Michiel en de rest van de Thermofysiologie-groep bedanken. Ik vond het erg leuk om samen te werken. Bedankt voor de betrokkenheid, gezellige praatjes en flexibiliteit. Jullie hebben een mooie studie afgerond en ik ben blij dat ik kon bijdragen. Frieder and Jakob, your enthusiasm about the circadian rhythm is praiseworthy. It was great to get the opportunity to dive into this topic and work with you both. Together with your incredible work we have published a very nice paper. Marlies and Evelyn, I am very glad we could make a story of the resveratrol paper! Thanks for all your help. Evelyn, thanks for staying connected, even from the other 
side of the world. Wish you all the best.

Furthermore, I like to thank all the members of the Energise consortium for the collaborations. Our biannual meetings were nice to cooperate, network and get motivated for science. All the nice chats with the members from Wageningen, Amsterdam and Leiden. Montserrat, I liked our trips and chats during the lipid droplet conference!

Alle andere (oud) collega's van DMRG (SHOCk), Alex, Bas, Carlijn, Charlotte, Daniel, Dennis, Dirk, Domenico, Edmundo, Elena, Eva, Jan, Jeremy, Jeroen, Julian, Kay, Lena, Lucas, Maaike, Manon, Niels, Pandi, Rodrigo, Sarah, Silvie, Sten, Stephanie, Tineke, Vera, Yvo en Yvonne, bedankt voor de samenwerking, alle hulp, en gezelligheid binnen en buiten het werk. Samen lunchen, borrels, uitjes. Ook voor alle sportactiviteiten buiten het werk. Anna, jij motiveerde mij altijd om toch te gaan spinnen. Emmani, wat gek dat jij nu ook weg bent bij de groep. Jij was altijd een vaste kracht in het (cel)lab. Gezellig dat we samen het BKO traject door konden gaan en ik wens je veel succes met je carrière. Ciarán, your passion for science is contagious. Thank you for that!

Special thanks for all the bachelor and master students, for their hard work and enthusiasm. It was great to supervise you all and I wish you all the best in the future. I want to thank all other colleagues from VBW and HB for their engagement, support, chats, 'gezelligheid', day-outs, sporty activities and weekends away. As far as I know, I have never missed a HB/VBW weekend and have good memories from them. Hopefully these trips will continue in the future.

Alle mensen van Galou, bedankt voor jullie Limburgse gezelligheid de afgelopen jaren! Ik vond het ontzettend leuk om met jullie samen te zingen, mooie optredens te verzorgen en achteraf een drankje te drinken. Ik hoop snel weer naar het mooie Heuvelland terug te keren om weer een concert van jullie bij te wonen.

Mijn lieve goede vrienden: Suzanne, Anniena en Pascal. Ik ken jullie al meer dan 10 jaar! Jullie steun was zeer belangrijk tijdens mijn promotie. Ondanks dat ik zo ver weg 


\section{Appendix}

ben gaan wonen en erg druk was met mijn PhD, waren jullie altijd vol begrip. Suuz, onze uitstapjes en gesprekken geven mij altijd zoveel nieuwe energie! Samen hebben we leuke uitstapjes gemaakt en ik hoop dat we die weer in de toekomst kunnen plannen. En dan met veel gesprekken over van alles en nog wat. Misschien kunnen we ooit eindelijk dat fristi ijsje proberen in Ermelo. Anniena, we hebben samen al veel meegemaakt en ik ben blij dat we via bellen en skype nog zoveel contact kunnen houden en dat waardeer ik enorm! Ik hoop snel nog een keer van je kookkunsten te kunnen genieten in Rotterdam. Pascal, je bent altijd in om gezellig een wijntje te drinken. Door jou word ik nog op de hoogte gehouden van de nieuwste films en wat er allemaal gebeurt in Almere.

Lieve familie, bedankt voor jullie steun, interesse en gezelligheid de afgelopen tijd. Lieve schoonfamilie, wat leuk dat ik jullie heb mogen leren kennen de afgelopen jaren. Jullie hebben mij vanaf het begin warm ontvangen en bedankt voor jullie steun en gezelligheid. Heerlijk om af en toe in Zeeland uit te waaien tussen alle drukte door.

Mijn lieve ouders, Martin en Hilly. Zonder jullie onvermoeibare steun had ik hier nooit gestaan. Zelfs toen ik besloot wéér te verhuizen, stonden jullie voor mij klaar. Bedankt dat jullie er altijd voor mij zijn, in mij geloven en trots op mij zijn. Lieve Vonne, je bent een geweldige grote zus. Bedankt voor al jouw steun en hulp de afgelopen tijd. Ik mocht jouw paranimf zijn tijdens jouw promotie en nu mag je ook mijn promotie meemaken. Je hebt altijd begrepen wat ik door moest maken tijdens mijn PhD en kwam altijd met gepast advies. Ik hoop jou, Iwan, Tobias en Livia weer snel te zien!

Lieve Casper, wat fijn dat ik jou heb mogen leren kennen. Wie had ooit verwacht dat ik in Maastricht een Utrechter tegen zou komen? Bedankt voor jouw ongelofelijke steun de afgelopen 4,5 jaar. Als ik het even niet meer zag zitten was jij altijd degene die mijn hand weer pakte en mij omhoog trok. Bedankt dat je in mij gelooft in alles wat ik doe. We hebben samen mooie reizen gemaakt de afgelopen jaren en elkaar gesteund tijdens onze promotietrajecten. Ik kijk uit naar onze toekomst en nieuwe avonturen samen. Ik hou van je. 
Dankwoord 



\section{List of publications}

Harmsen JF, van Polanen N, van Weeghel M, Wefers J, Hoeks J, Vaz FM, Pras-Raves ML, van Kampen AHC, Schaart G, van Moorsel D, Hansen J, Hesselink MKC, Houtkooper RH, Schrauwen P. Circadian misalignment disturbs the skeletal muscle lipidome in healthy young men. FASEB J. 2021 Jun;35(6):e21611. doi: 10.1096/fj.202100143R.

van Polanen N, Zacharewicz E, de Ligt M, Timmers S, Moonen-Kornips E, Schaart G, Hoeks J, Schrauwen P, Hesselink MKC. Resveratrol-induced remodelling of myocellular lipid stores: A study in metabolically compromised humans. Physiol Rep. 2021 Jan;9(2):e14692. doi:10.14814/phy2.14692.

Houzelle A, Jörgensen JA, Schaart G, Daemen S, van Polanen N, Fealy CE, Hesselink MKC, Schrauwen P, Hoeks J. Human skeletal muscle mitochondrial dynamics in relation to oxidative capacity and insulin sensitivity. Diabetologia. 2021 Feb;64(2):424-436. doi: 10.1007/s00125-020-05335-w. Epub 2020 Nov 30.

Daemen S, van Polanen N, Hesselink MKC. The effect of diet and exercise on lipid droplet dynamics in human muscle tissue. J Exp Biol. 2018 Mar 7; 221(Pt Suppl 1). doi: 10.1242/jeb.167015. Review.

Hesselink MKC, Daemen S, van Polanen N, Gemmink A. What are the benefits of being big? J Physiol. 2017 Aug 15; 595(16):5409-5410. doi: 10.1113/JP274723

van Polanen $\mathbf{N}^{*}$, Daemen $\mathrm{S}^{*}$, Bilet L, Phielix E, Moonen-Kornips E, Schrauwen-Hinderling VB, Schrauwen P, Hesselink MKC. Post-exercise changes in myocellular lipid droplet characteristics of young lean individuals are affected by circulatory non-esterified fatty acids. Accepted in the American Journal of Physiology-Endocrinology and Metabolism

*These authors contributed equally

Moonen MPB, van Polanen N, Rense P, Jörgensen JA, Moonen-Kornips E, Beckers M, Hoeks J, Wierts R, van de Weijer T, Havekes B, Plat J, Schrauwen P, van Marken Lichtenbelt WD. Acute cold exposure leads to increased levels of triglycerides during mixed-meal tests in young healthy subjects. In preparation 



\section{About the author}

Nynke van Polanen was born on the $7^{\text {th }}$ of June, 1990 in Almere, the Netherlands. She graduated from secondary education in gymnasium (university preparatory education with Latin) and received an International Baccalaureate diploma in English. After graduation, she was enrolled in the bachelor program Nutrition and Health at Wageningen University \& Research. During her bachelor thesis she did a literature study on the relation between ADHD and the infant gut microbiota. After obtaining her Bachelor of Science degree, she continued

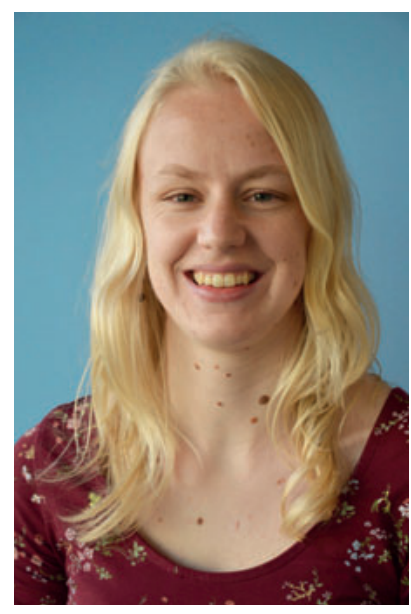
her education with a two-year master program Molecular Nutrition and Toxicology at Wageningen University \& Research. She performed her master thesis in the group of Prof. Keijer at the Human and Animal Physiology department in Wageningen. She investigated the effect of silencing $M f n 1$ and Dnm1l on mitochondrial dynamics and related mitochondrial functioning in C2C12 myoblasts. For her internship, Nynke travelled to the United States and performed biomedical research in Dallas at the Scherer's lab, Touchstone Diabetic Center, University of Texas Southwestern. She investigated the effects of adiponectin on tubular epithelial cell metabolism in mice. She received her master's degree thereafter in 2015. In September 2016, she started her PhD within the Diabetes and Metabolism Research Group under supervision of Prof. Hesselink, Prof. Schrauwen and Dr. Gemmink, at the department of Nutrition and Movement Sciences at Maastricht University. The research conducted during this period, as described in this dissertation, was focused on myocellular lipid droplets in relation to insulin sensitivity. She used fluorescent microscopy techniques to study lipid storage and high-resolution respirometry to study mitochondrial function in human skeletal muscle in different human intervention studies. In July 2021, she started as a postdoctoral research assistant in mitochondrial disease in the group of Prof. Poulton at the University of Oxford, in the UK. 


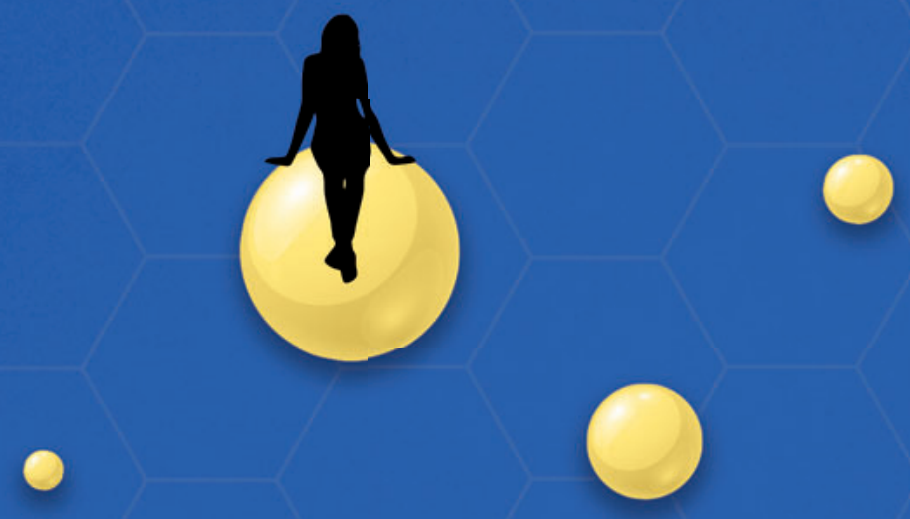

